\title{
DESIGNING AIR TRANSPORT NETWORKS THAT SERVE SPARSE DEMANDS
}

\author{
BY \\ Abdulaziz A. Khayat \\ B.S., The University of Wisconsin-Milwaukee, 1983 \\ M.S., The University of Wisconsin-Milwaukee, 1985 \\ A thesis submitted in partial fulfilment of \\ the requirements for the degree of \\ Doctor of Philosophy \\ in \\ The Faculty of Graduate Studies \\ Department of Civil Engineering
}

we accept this thesis as conforming to the required standard.

The University of British Columbia

December, 1993

(C) Abdulaziz A. Khayat, 1993 
In presenting this thesis in partial fulfilment of the requirements for an advanced degree at the University of British Columbia, I agree that the Library shall make it freely available for reference and study. I further agree that permission for extensive copying of this thesis for scholarly purposes may be granted by the head of my department or by his or her representatives. It is understood that copying or publication of this thesis for financial gain shall not be allowed without my written permission.

(Signature)

Department of Ciril Engineering

The University of British Columbia

Vancouver, Canada

Date $\operatorname{Tan} 18,1994$ 


\section{$\underline{\text { ABSTRACT }}$}

The aim of this thesis is to formulate a balanced routing strategy for constructing efficient airline networks in situations where travel demand is sparse. The strategy must strike a balance between the various objectives of both the airline operator and the passengers. Integer programming was used to develop three routing algorithms. The algorithms were then used to create three efficient air networks. The networks were designed to attain specific objectives. The first network minimized the total cost of airline operations. This was accomplished by consolidating the traffic demands at a few efficient hub locations to reduce the unit cost of operations, and by reducing the minimum number of required flights that meet all traffic demands. The second network minimized the cost of travel of the passengers by reducing their travel and schedule delay times. The third network was designed to minimize the total combined cost of both the airline and the passengers. This was done by minimizing the airline unit costs of operations, schedule delays, and travel times.

As a case study, the domestic air transportation network of the Kingdom of Saudi Arabia were examined. The routes and links between the different airports in the network were restructured. The study showed that the algorithms were successful in achieving their design goals. The study also showed that the attributes of air transport networks influence both the passengers' travel cost and the airline operating cost. The total cost and network structure are not independent. Hence, air transport networks must be developed in such a fashion that total costs are minimized and travel service within the network maximized. 


\section{TABLE OF CONTENTS}

Abstract $\quad$ ii

Table Of Contents

List Of Figures $\quad$ vi

List Of Tables $\quad$ viii

Acknowledgements $\quad$ ix

\section{INTRODUCTION}

1.1 .0 Background 1

1.2.0 Routing Strategies $\quad 3$

1.2.1 Hub and Spoke Strategy $\quad 4$

1.2.2 Point to Point Connection Strategy 6

$\begin{array}{ll}\text { 1.3.0 Problem Definition } & 8\end{array}$

$\begin{array}{ll}\text { 1.4.0 Thesis Objectives } & 8\end{array}$

1.5.0 Description of The Study 10

$\begin{array}{ll}\text { 1.6.0 Case Study } & 10\end{array}$

2. LITERATURE REVIEW 12

2.1.0 The Split Routing Strategy 15

2.1.1 Discussion of The Model 17

2.2.0 Heuristic Frequency Planning Model 18

2.2.1 Discussion of The Model 25

2.3.0 The Hierarchical-Hub (Hierhub) Model For Airline Networks 26

2.3.1 Discussion of The Model 33

2.4.0 Other Work 34

$\begin{array}{ll}2.5 .0 \text { Summary } & 35\end{array}$

3. METHODOLOGY 37

3.1.0 Linear And Integer Programming 37

3.1.1 Assumptions 39

3.1.2 Graphical Presentation of Linear Programming $\quad 41$

3.1.3 Solution Concept $\quad 43$

3.1.4 Integer Programming $\quad 48$

3.2.0 Formulation of The Problem. $\quad 49$ 
3.2.1 Airline's Network Design $\quad 52$

3.2.2 Passenger's Network Design 53

3.2.3 Combined Airline \& Passenger Network Design 59

3.2.4 Discussion And Validation of The Formulations $6 \mathrm{C}$

3.3.0 Discussion of The Methodology 66

3.4.0 Computerizing The Formulation 68

4. THE COMPUTER PROGRAM $6 \mathrm{~S}$

4.1.0 The Program 6S

4.1.1 Input Data, And Create Data And Problem Formulation Files 71

4.1.2 View And Edit Existing Data File 74

4.1.3 Read Existing Data File And Create Problem Formulation File 75

4.1.4 Analyse Network Designs $\quad 75$

4.1.5 Performance Indicators And Analysis Data 76

4.1.6 Sample Output 79

5. The CASE STUDY 81

$\begin{array}{lc}\text { 5.1.0 Existing Network Conditions } & 81\end{array}$

5.1.1 The Structural Shape of The Existing Network $\quad 82$

5.1.2 Operational Characteristics of The Existing Network 84

5.2.0 Case study Results $\quad 88$

5.3.0 Analysis of Results $\quad 88$

5.3.1 Efficient Airline's Cost Network 92

5.3.2 Efficient Passenger's Cost Network 97

5.3.3 Efficient Combined Cost Network 103

$\begin{array}{ll}\text { 5.4.0 Summary } & 107\end{array}$

$\begin{array}{ll}\text { 6. COCNCLUSIONS } & 10 \mathrm{~s}\end{array}$

7. FURTHER RESEARCH 113

REFERENCES 115

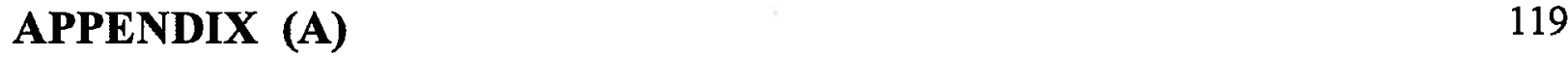


$\begin{array}{llr}\text { APPENDIX (B) } & 127\end{array}$

$\begin{array}{ll}\text { APPENDIX (C) } & 179\end{array}$

$\begin{array}{llr}\text { APPENDIX (D) } & 189\end{array}$ 


\section{LIST OF FIGURES}

1.1 Airline Routing Strategies 2

2.1 Illustration of Split Routing $\quad 16$

$\begin{array}{lll}2.2 & \text { Flowchart of The Frequency Planning Model } & 20\end{array}$

2.3 Patterns of Hub And Spoke Network $\quad 27$

2.4 Example of The E Matrices

3.1 Graphical Presentation of Linear Programming 43

3.2 Convex And Nonconvex Feasible Regions 45

3.3 The Two Convex Parts of The Feasible Region 46

3.4 Beesley's Method For Estimating The Value of Time 55

3.5 Finding The Optimal Economic Cost of Travel 60

3.6 Example 1, Network Problem $\quad 62$

3.7 The Traditional Urban Planning Solution $\quad 63$

3.8 The Loop Solution $\quad 64$

3.9 The Third \& Fourth Possible Solutions $\quad 65$

3.10 The Optimal Solution For Example 1 65

3.11 Example 2, Network Problem $\quad 66$

3.12 The Optimal Solution For Example 2 66

$\begin{array}{lll}4.1 & \text { Program Flowchart } & 70\end{array}$

4.2 Input Screen, Typical Example $\quad 73$

5.1 The Existing Domestic Air Transport Network of The Kingdom of Saudi Arabia 83

5.2 Estimated Load Factors Distribution In Existing Network 84

5.3 Estimated Number of Flights Distribution In The Existing Network 85

5.4 Estimated Schedule Delay Distribution In The Existing Network 86

5.5 Estimated Cost Per Passenger-Mile Distribution In Existing Network 87

5.6 Efficient Airline's Cost Network $\quad 89$

5.7 Efficient Passengers' Cost Network 90

$\begin{array}{lll}5.8 & \text { Efficient Combined Cost Network } & 91\end{array}$

5.9 Estimated Load Factors Distribution In Efficient Airline Network 92

5.10 Estimated Number of Flights Distribution In Efficient Airline Network 93

5.11 Estimated Schedule Delay Distribution In Efficient Airline Network 95

5.12 Estimated Cost Per Passenger-Mile Distribution In Efficient Airline Network 96 
5.13 Estimated Load Factors Distribution In Efficient Passenger Network

5.14 Estimated Number of Flights Distribution In Efficient Passenger Network

5.15 Estimated Schedule Delay Distribution In Efficient Passenger Network

5.16 Estimated Cost Per Passenger-Mile Distribution In Efficient Passenger Network

5.17 Estimated Load Factors Distribution In Efficient Combined Network

5.18 Estimated Number of Flights Distribution In Efficient Combined Network

5.19 Estimated Schedule Delay Distribution In Efficient Combined Network

5.20 Estimated Cost Per Passenger-Mile Distribution In Efficient Combined Network

6.1 Cancelled Links From The Existing Network In The Efficient Networks

7.1 Required Models For Efficient Airline Operations 


\section{LIST OF TABLES}

5.1 Attributes of Existing And Design Networks 


\section{ACKNOWLEDGEMENTS}

I would like to express my deep appreciation to General Said Yousif Amin and Dr. Waddah $\mathrm{K}$. Alem for giving me the opportunity to pursue my academic goals. I am also grateful to the members of my examining committee and in particular to Dr. Frank Navin for his guidance, helpful insights, and patience during the course of this study.

Special thanks are also extended to my dear friend Dr. Abdulaziz Al-Madi, my family specially my wife, and all my good friends specially those at the Bechtel corporation, I.A.P., and U.B.C. for their support of my search for knowledge. 


\section{INTRODUCTION}

\subsubsection{Background}

Transportation networks are developed to move people and goods. The total cost of travel around a network depends on the capital costs of the fixed facilities and the operating cost within the network. The attributes of the network influence both the capital cost and operating cost. The total cost and network structure are not independent but must be developed in such a fashion that total costs are minimized and travel service within the network maximized. The general problem addressed in this study is that of developing an efficient network when demand is sparse and spread over a large geographical area. The problem reduces to that of sparse networks. Such a problem is interesting to study because as will be seen later in the literature review chapter of this thesis, no research has been specifically published dealing with sparse demand levels. Most of the published work on optimizing airlines operations has concentrated on making existing routes more profitable by optimizing crew and flights scheduling.

Air transport services are characterized by high fixed costs at airports and operating costs that depend on the routing structure and travel demand. In networks with low demand for travel, operating costs are thought to become even more critical and the shape of the network has greater impact on the final per unit cost of travel. The network's structural shape is dictated by the airline routing strategy. The most commonly used strategies are shown in Figure 1.1. These strategies are : the Linear Point to Point Connection Strategy and the Hub and Spoke Strategy. 


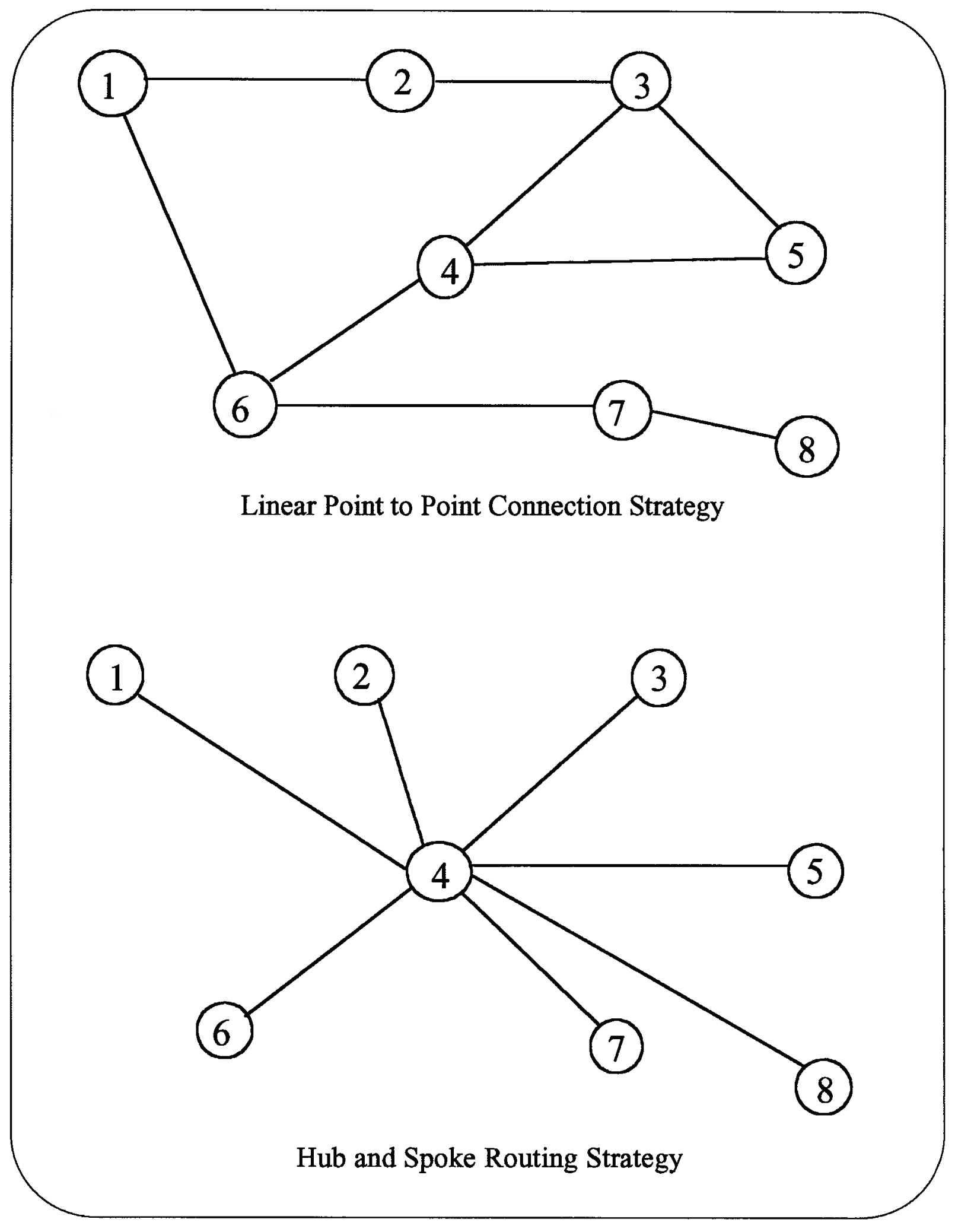

Figure 1. 1 Airline Routing Strategies 
As it will be shown later, airline routing strategies fall into two categories, they are : Point to Point Connection or Hub and Spoke. Air transport networks having high degree of direct point to point connectivity and sparse travel demands are often costly to operate. Hub and spoke networks tend to increase travel time and its associated cost. Yaw Jeng ${ }^{1}$ has shown that a sound routing strategy can increase the air carrier's operational efficiency and provide more convenience for the air travellers. Therefore, in sparse travel demands situations a balanced routing strategy must be formulated to simultaneously consolidate travel demand and reduce travel time in order to minimize the total unit cost of travel. The importance of a good routing strategy is more evident when dealing with large geographical areas with sparse travel demand levels. The air routes that develop in such situations are normally characterized by low levels of demands and low load factors on the aircraft. In addition, the frequency of service is low and the size of the aircraft used on such networks tend to be small. Smaller aircraft have been found by Schwieterman ${ }^{2}$ to be less cost effective per seat mile supplied relative to larger aircraft as flight distance increases. On short routes however, smaller aircraft have a relative advantage over larger ones. To provide a better understanding of the two strategies, the following will be a detailed discussion of their evolution, attributes, advantages, and disadvantages.

\subsubsection{Routing Strategies}

Through the years different routing strategies have been developed to fulfil some specific needs of moving people and goods between demand locations. In the U.S., the earliest routes were determined by airmail requirements. The early airmail routes were established 
between large cities and areas of high population concentrations. Not long after, transcontinental routes were developed to link the entire country. Prior to the airline Deregulation Act of 1978 in the U.S., routes were regulated by the government and the airline's routing strategy was accordingly dictated. A common strategy in most public transportation systems at that time was to establish linear route structures with relatively closely spaced stops. This strategy has also been used in air transportation, where it is called "hedgehopping". However, the costs and passengers inconvenience entailed in making stops along the route is much greater in air transportation than it is in other modes ${ }^{3}$. Also the preference of passengers for a minimum number of stops along the way is very strong ${ }^{4}$. These preferences combined with deregulation has caused the U.S. route system to be mostly shaped by competition between the airlines and the new hub and spoke routing strategy has come to existence. Carriers have taken deregulation as an opportunity to redeploy their resources and to make major changes in their route networks 5 .

\subsubsection{Hub And Spoke Strategy}

A hub and spoke network can be thought of as a radial system of roads such as existed in older cities. The central city is equivalent to the hub of a wheel and the routes radiating from it are the spokes that serve the outlying suburbs. In this type of network, the hubs act as collection and dissemination points where the traffic demands are consolidated and an indirect connection through the hub city between many city pairs that by themselves cannot generate enough traffic to justify direct flights is permitted ${ }^{6}$. Flights converge on a hub at approximately the same time, this convergence of flights is referred to as "bank" or "complex". Each bank 
lasts from the time the first inbound flight arrives at the hub until the last outbound flight has departed ${ }^{7}$ Flights are normally scheduled in such a way that passengers can transfer between flights on a bank after an adequate time to allow passengers and baggage to be exchanged.

Wheeler ${ }^{8}$ listed the advantages and disadvantages for hub and spoke networks. The main advantages are as follows:

- A hub and spoke network provides high frequency service to a large number of low density city pairs. This type of network allows a carrier to market many origin and destination possibilities to each spoke city. This strategy allows the carrier to compete in many markets while using relatively few resources. The resulting high frequency service is more desirable because it is attractive to business passengers and also it increases the carrier's chance of achieving top line display in the computer reservation system (CRS) 9

- If there is high demand for non-stop service, a hub and spoke operator can realize higher than average fares on a non-stop route that it monopolizes.

- The large number of origin and destination markets that are normally served by this type of network allows a hub and spoke operator to be less dependent on any particular market or group of markets. Hence, the economic effects of traffic demand fluctuations in a particular market will be minimized by this type of network due to the large number of other markets it usually serves.

- $\quad \mathrm{A}$ hub and spoke operator can normally market a large number of possible itineraries and thereby increase the number of passengers that he can serve. Also passengers tend to like using the same carrier for the entire trip if the trip involves a transfer. Hence, because of the large possible itineraries, the operator will be most likely able to serve these transfer passengers. 
This is highly desirable because the carrier can then keep a connecting passenger's full fare, rather than a portion of it.

- The large number of possible routing of aircraft and crew at hubs permits greater use of equipments and labour, as well as increased operational flexibility.

According to Wheeler ${ }^{10}$ the main disadvantages of using hub and spoke networks are as follows:

- A hub and spoke network contributes to congestion and delay at major airports and in the traffic sectors that serve these airports. Although in most countries specially in the United States these problems are largely the result of the narrowing gap between system capacity and the volume of aircraft movements, they are exacerbated by the self imposed delay that results from the "banking" of flight schedules by hub carriers for the purpose of connecting flights.

- The consolidation of a carriers operations at its hub results in over use of terminal staff, gates, and equipments. This network type consequently has higher departure cost than linear networks.

- Finally, poor weather conditions at a hub airport can result in increased delays and costs through the system because of reaccommodation of misconnected passengers, prevention of illegal scheduling of crew, and other operational problems. 


\subsubsection{Point To Point Connection Strategy}

In this strategy as the name implies, long-haul multistage connections between large cities are normally provided by the carrier. For example, Figure 1.1 shows that passengers travelling from airport 1 to airport 8 must make three stops along the way. The figure also shows that the route system does not have any dominant focus. Almost all airports in the network have the same number of links connected to them. Oum and Tretheway ${ }^{11}$ indicated that this route structure is sometimes referred to as a "Linear route system". The reason behind this name is the original government awards of the components of the route system tended to be straight line routes. For example, the airline may initially have been awarded the right of service from 1 to 2 to 3 to 5 . It may have been subsequently awarded the service right from 1 to 6 to 7 to 8 . The remaining routes were most likely awarded later in time.

Long stage lengths require larger aircraft for range, and because of the lack of feeder traffic on this type of network the service frequency and passenger load factors tend to be low which increases the cost per passenger unless the city pairs themselves are high traffic generators. The low service frequency tend to increase the average schedule delay of passengers. Schedule delay is defined as the difference in time between when a person would like to make a trip and the time he is constrained to make the trip because of the airline's schedule $^{12}$. Long travel distances may require overnight layovers, which reduce aircraft utilization rates. Depending on the size of network, aircraft servicing and apron services may have to be performed at various locations. Finally, it is well known that major linear routes between important cities tend to suffer from intense competition and significant discounting ${ }^{13}$. 


\subsubsection{Problem Definition}

If properly developed, hub and spoke networks are more cost efficient for the airline especially when demand is sparse because they consolidate the traffic at the hubs and reduce the minimum number of required flights. However, they tend to increase travel circuity. Circuity is defined as the extra distance needed to serve an origin destination (OD) pair by hub and spoke rather than point to point operation ${ }^{14}$. As a result of increased circuity, hub and spoke networks increase both the travel time and its associated cost which is incurred by the passengers. In contrast, point to point connection networks tend to do the opposite. They reduce the travel time and its associated cost because of the direct connections. However, they are less cost efficient for the airline than the hub and spoke type specially when demand is sparse. The lack of feeder traffic on this type of network reduces passenger load factors unless the OD pairs themselves are high traffic generators. The reduced load factors increase the cost of operations per passenger mile because of the high fixed cost of service that is normally allocated to airline operations. Also, the increased direct connectivity increases the minimum number of required flights which in return increases the operating cost of the airline. Evidently, serving large areas with sparse travel demands constitutes a unique problem. The problem here is to formulate a balanced routing strategy which will minimize the airlines operating cost by consolidating the demands at some locations in the network. Also the strategy must minimize the passengers travel times and satisfy all the service demands. 


\subsubsection{Thesis Objectives}

Given the two routing strategies, and the complexities of demand in large sparse networks it becomes important to develop a balanced strategy for routing aircraft throughout the network. Therefore, the objective of this thesis is to develop integer programming algorithms that will yield efficient routing strategies for sparse demand levels. The strategies will then be used to develop efficient air network structures. The structures must be developed in such a way as to minimize the travel unit costs of both the carrier and the passengers. The carrier's cost will be minimized by consolidating the travel demands at some ideal hub locations in the network. These ideal locations will be identified by the algorithms in accordance to the demand distribution throughout the network and the ability of these locations to generate and attract traffic. The passengers' cost will be minimized by routing the traffic on routes that reduce travel times and schedule delays. The number of transfers that a passenger has to go through will be limited to one. Limiting the number of transfers to one is practical in two ways. First, it is realistic since in practice the majority of travellers try to limit their transfers. Second, this limitation greatly reduces the number of possible link combinations between any two airports in the network. As a result, this limitation reduces the number of variables and constraints and speeds up the computations. The algorithms must also be flexible enough to allow the user to model some real life constraints and needs. For example, a specific link between any given two nodes can be included or excluded in the final network structure for any given reason. Also, the algorithms must be able to deal with the possibility or impossibility of developing a certain airport as a hub in the final network structure. This is necessary because some airports do not have the gate capacity that will allow them to be developed as hubs. Finally, the routing 
algorithms will be built into a computer package that will develop the complete integer programming formulations of the efficient air network structure. The package will also evaluates the network's effects on the airline operations and passengers services in terms of load factors, operating cost, flight frequencies, and travel times.

\subsubsection{Description Of The Study}

Some form of integer programming will be employed. The thesis will develop a model which will link air transportation demand levels to an efficient routing strategy. Generalized cost functions will be developed and used as disutility measures of all possible routes between the various demand locations to develop the efficient routing strategy. The cost functions will include elements of demand levels, passenger cost of travel time and schedule delay, airlines cost per passenger, and elements of the two routing strategies. Assuming that the airline can provide enough flight capacity to satisfy the demands between all the different origin and destination pairs, the model constraints will then be limited to providing a route between any pair. All possible links between the different demand locations according to the hub and spoke and point to point connection strategies will be used in the generalized cost function as the decision variables of the model. However, only links that contain a maximum of one transfer point connecting any pair of demand locations will be considered. This restriction is used to reduce the large number of possible route combinations if more than one transfer is considered. The model will also be flexible and will allow the user to include or exclude any particular link in the network according to his/her judgement. 


\subsubsection{Case Study}

Initially, this thesis attempted to study the air transport networks of Australia and northern Canada. However, this was not possible due to the lack of publicly available data elements of these networks. Alternatively, the domestic air transportation network of Saudi Arabia which consist of 25 airports that have sparse demand levels between the majority of these airports was selected as a case study. The routes and links between the different airports in the network were examined and restructured. This particular network was selected as a case study because it represents a unique opportunity since the network size is large, demand is sparse, and there is no other fast alternative of mass travel modes.

During the planning phase of this network, the network plan placed strong emphasis on objectives such as the country's political integration, the establishment of direct links between all population centres, and the social well-being of the population. As a result, all the Kingdom's centres of population are directly connected to the air transport system by their own airports. The majority of these airports are directly connected to one another even though demand levels do not necessarily justify direct connections. This high degree of direct connectivity and the low travel demands resulted in lowering the load factors on the majority of flights. 


\section{LITERATURE REVIEW}

Most of the published work in the area of optimizing airlines networks has concentrated on developing optimum schedules for a predetermined set of routes. This is possibly so because airline routes were regulated by the government. Thus, the majority of authors have treated airline routing and scheduling as one single process. The separation of these processes is important since it has been found that the efficient selection of routes leads to more profitable scheduling ${ }^{15}$. Hence, the determination of the sequence of stops for each aircraft through the network of cities prior to scheduling is a critical function. Efficient routing selection is even more critical when dealing with sparse demand, because it can consolidate passengers from different origins destined to one city through a hub airport and ultimately increase the overall aircraft load factor. Aircraft load factor is defined as the percentage of occupied seats, which is a measure of capacity utilization of the aircraft. Therefore, this thesis will treat airlines routing and airlines scheduling as a separate but highly related processes. Airlines routing which is the main concern of this thesis is defined as the sequence of cities where one aircraft lands. A schedule is constructed when the routes are assigned specific departure times for each city and is evaluated for constraints and factors not included in the route construction process such as crew scheduling, terminal operation requirements, and maintenance requirements of aircraft. Airline scheduling process normally encompasses complicated tasks of, synthesizing a multiple of interrelated political, economic, social, legal, and technical factors, to produce a balanced pattern of flights with a timetable that is consistent with the airline's objectives and constraints. In practice, route construction, traffic forecasting, and operational evaluations are the major activities that form the nucleus of the scheduling process. Traffic forecasts are normally 
prepared by using information on the general economic conditions, competitive schedules, and fare structures. This forecasting process estimates the expected traffic by flight segment as an average for a given period. Then, an initial set of routings is constructed by combining the information on forecasted traffic with the previously flown timetable and routes to produce a preliminary schedule. A new forecast is then made using the preliminary schedule along with the other inputs. Based on identified operational inconsistencies and the previous demand, another set of routings is constructed. the process continues to iterates through these steps until the final, operational timetable is produced (Richardson) ${ }^{16}$.

Prior to the airline deregulation act of 1978 in the U.S. when routes were dictated by the government, most of the optimization work focused on determining the optimal airline scheduling scheme for the regulated routes. Gordon and De Neufville ${ }^{17}$ presented a fleet allocation model that optimizes the level of service of a given air transportation network by minimizing the total delay, $\mathrm{D}$, experienced by passengers being served by a fleet with a given number of aircraft over a given set of routes. The concept of schedule delay was introduced in this model as a measure of the value of a given frequency of service. Schedule delay was defined as the difference in time between when a person would like to make a trip and the time he is constrained to make the trip because of the inflexibility of the airline's schedule. If the load factor is fixed on the given routes, it was assumed that schedule delay could be represented with sufficient accuracy as proportional to:

$$
\left(1-p_{i}^{s}\right)^{-1 / s} ; s \geq 1
$$


where

$s=$ un determined constant that was assumed to be equal to 1 .

$p_{i}=$ the load factor on link $\mathrm{i}$ which is assumed to be fixed by the airlines.

Based on the above equation, the total schedule delay which is measured in passenger hours for all passengers was represented as:

$$
D=a \sum_{i} v_{i}\left(1-p_{i}^{s}\right)^{-1 / s} N_{i}^{-1}
$$

where

$a=$ proportionality constant that is assumed to be the same along every link.

$v_{i}=$ the volume of passengers on link $i$.

$N_{i}=$ the number of aircraft on route $\mathrm{i}$.

The model then basically minimizes the total schedule delay given by the above equation. However, the amount of service provided can not exceed the capability of the aircraft fleet, $S$, which is measured in units of available seat-hours over a given period of time. In practice, $S$ would be calculated form the knowledge of the average utilization, capacity, and cruise speed of the aircraft in the fleet. Hence, the model is subject to the following constraint:

$$
\sum_{i} N_{i} c t_{i} \leq S
$$

where

$c=$ the capacity of aircraft

$t_{i}=$ the total gate-to-gate or block time for an aircraft over route $\mathrm{i}$. 
The model can be solved for any specific patterns of demand, size of aircraft, network configuration and number of aircraft in the fleet. By manipulating these variables and solving the problem and then comparing the results of the solutions, it becomes possible to determine optimal values of these factors.

Dantzig and Ferguson ${ }^{18}$ formulated the original linear programming model that determine the optimal frequency of flights between each city pair to maximize revenue. Etschmaier ${ }^{19}$ related the airline demand to its frequency of service and the attractiveness of different aircraft types as well as to the general competitive position of that airline in the market. Kushige ${ }^{20}$ considered the case where flight frequencies are symmetrical on the routes but the demand is not.

After the Deregulation, a new trend in airline's routing has emerged. Airlines have taken deregulation as an opportunity to redeploy their resources and to allow market competition forces to shape their networks. Since then more work that specifically deals with airline' routing has been published. The following literature review includes some of the published works on airline routing.

\subsubsection{The Split Routing Strategy}

Jeng ${ }^{21}$ proposed what he called the split routing strategy. The strategy is primarily based on serving passengers demands by providing either a direct point to point connection or a connection through a hub airport according to the relative locations of origins and destinations. 
The costs to be minimized in this strategy include airline operating costs and passenger travel time costs. The key network parameters include demand level, network size, and number of cities or nodes served by the network. The demand is assumed to be homogeneous and constant throughout the network. And the airline network is structured in a circular configuration. The circular network configuration shown in Figure 2.1 assumes that all non-hub nodes are uniformly located along the circumference of a circle with a hub at the center. To apply this strategy, airline networks are approximated as circular network needing only the radius of a

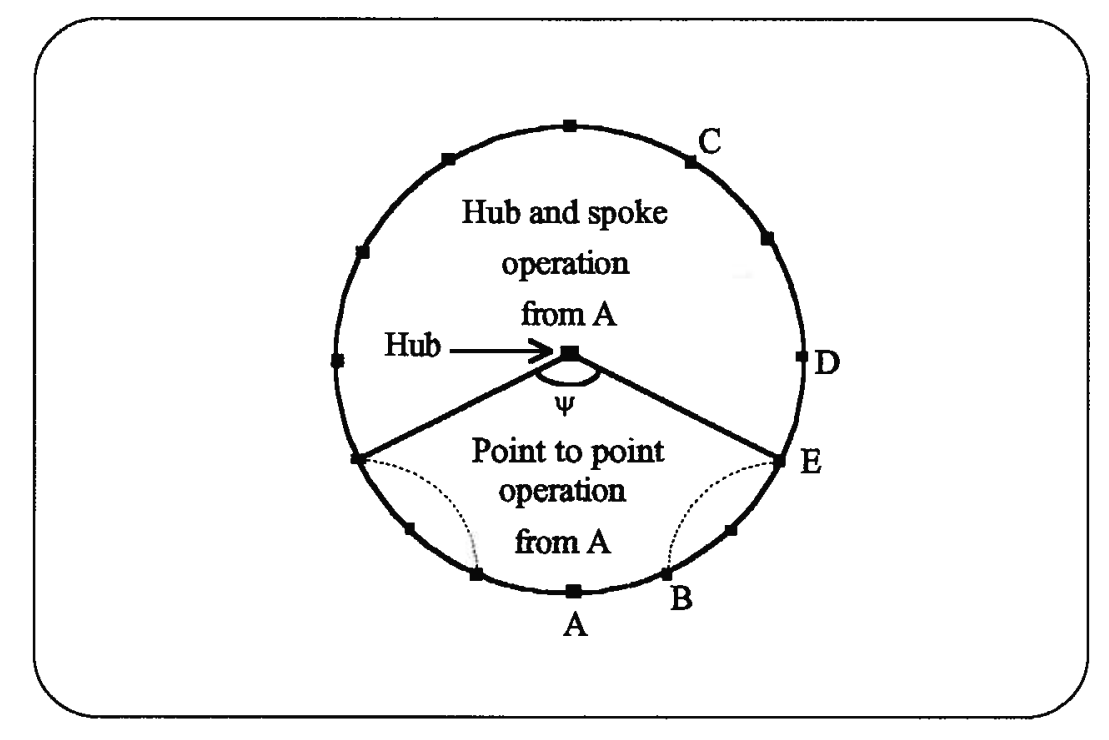

Figure 2. 1: Illustration of Split Routing

circle and the number of nodes. The radius of the circle can be calculated by averaging all the distances between non-hub nodes and the hub. According to the strategy, destinations are served by splitting them depending on their relative locations to the origin, with either point to point or hub and spoke operation. The main idea behind split routing is to reduce the circuity when serving any origin destination pair (OD). For example, the circuity to serve OD pair $A B$ 
in figure 2.1 by hub and spoke operation is much greater than the circuity to serve OD pair AC by the same operation. Therefore, it is reasonable and logical to serve the nodes closer to the origin with point to point and the others with hub and spoke operations. The problem then becomes finding the number of neighbouring nodes on each side of a non-hub origin that can be served by direct point to point operation such that the total cost of the service is minimized for both the airline and the passengers. This is solved by finding an optimum value for the split angle $\Psi$ as shown in figure 2 . 1 . The optimum value of $\Psi$ as derived by Jeng is presented by the following equation:

$$
\Psi^{*}=0.5 \pi \frac{P^{0.6}}{n^{0.2} d^{0.3}}
$$

Where :

$n=$ the number of nodes in the system

$p=$ Average demand per O-D pair (Passengers / day)

$d=$ Radius of circular network (mi)

$\Psi^{*}=$ The optimum split angle in radians

\subsubsection{Discussion Of The Model}

Jeng's study may provides good understanding of the trade-offs and cause-effect relationships among key network components and their impacts on the shape of the network. However, the simplified assumptions used in the study such as; the homogeneity of the demand, the inelasticity of the demand with respect to time and cost, and the circular shape of the network with one single hub are too restrictive for actual applications. Hence, the accuracy of its results specially those that relate to the optimum shape of the network is questionable. 
Also, serving sparse demand requires the consolidation of that demand in order to increase the load factor to minimize the cost per passenger, the model provided in this study does not has the mechanism to do so. In this method traffic may be consolidated at the single hub in the network but only in accordance to the relative geographical locations of the origin and destination.

\subsubsection{Heuristic Frequency Planning Model}

Ghobrial, Balakrishanan, and Kanafani ${ }^{22}$ developed a two-phase heuristic model for airline frequency planning and aircraft routing for small size airlines. The first phase of the model which is the most relevant to this thesis yields a frequency plan by using an economic equilibrium model between passenger demand for flying a particular route and airline's supply of flights on that route given the operating characteristics of aircraft flown. According to the authors, a frequency plan specifies the configuration of the network (non-stop or multi stop routes), frequency of service, and the type of aircraft assigned to each flight. The model however assumes only one aircraft type will be used for all flights. The second phase uses a time-of-day model to develop an assignment algorithm for aircraft routing. Together, both phases are referred to as the schedule construction process.

The model is built on the assumption that airlines tend to compete against each other by providing more flight frequency. This is so because airline's traffic share of a given market tend to increase as its frequency share in the market increases. Therefore, while airlines try to minimize their operating cost by flying the minimum number of flights in the market, their flight frequency competition pushes them to operate at their break-even load factors. Given 
individual airline's price and operating cost, market equilibrium is reached when each airline operates at its break-even load factor subject to indivisibility of flights and assuming a reasonable return on investment ${ }^{23}$. Given a set of origin-destination markets, a set of candidate routes (i.e. possible connecting routes for each origin and destination pair while keeping in mind that a route can be composed of a single or multiple links, and any link in the network could serve one or more routes connecting different city-pairs.), aircraft types, yield and operating cost functions, the objective of the model is to find a set of supply decisions. These decisions include aircraft type, frequency, and routings which achieves a certain goal such as maximizing airline profits.

Figure 2.2, shows the flowchart of a computer based solution algorithm which was developed by Ghobrial etal to incorporate a set of modules that constitute the frequency planning model. As shown in the figure, a set of candidate routes is defined by the user for each city-pair in the network. These routes represent the possible ways in which service can be provided between them (i.e. non-stop and multi-stop). The basic requirement for the network representation is to ensure a conservation of flow at each node. This can be accomplished by the following constraints:

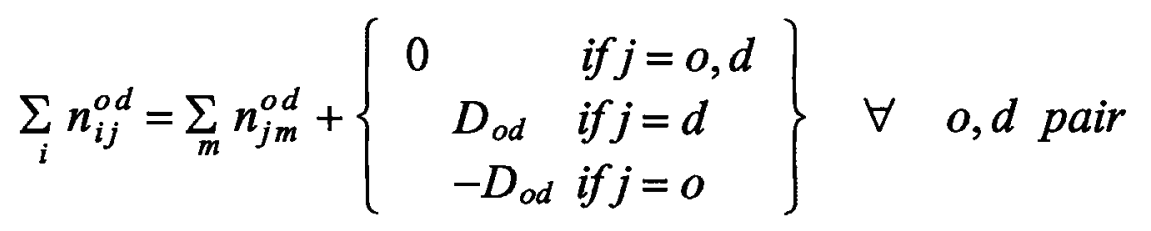

where :

$n_{i j}^{o d}=\mathrm{O}-\mathrm{D}$ traffic using link id.

$D_{o d}=$ O - D traffic (demanded at d) 


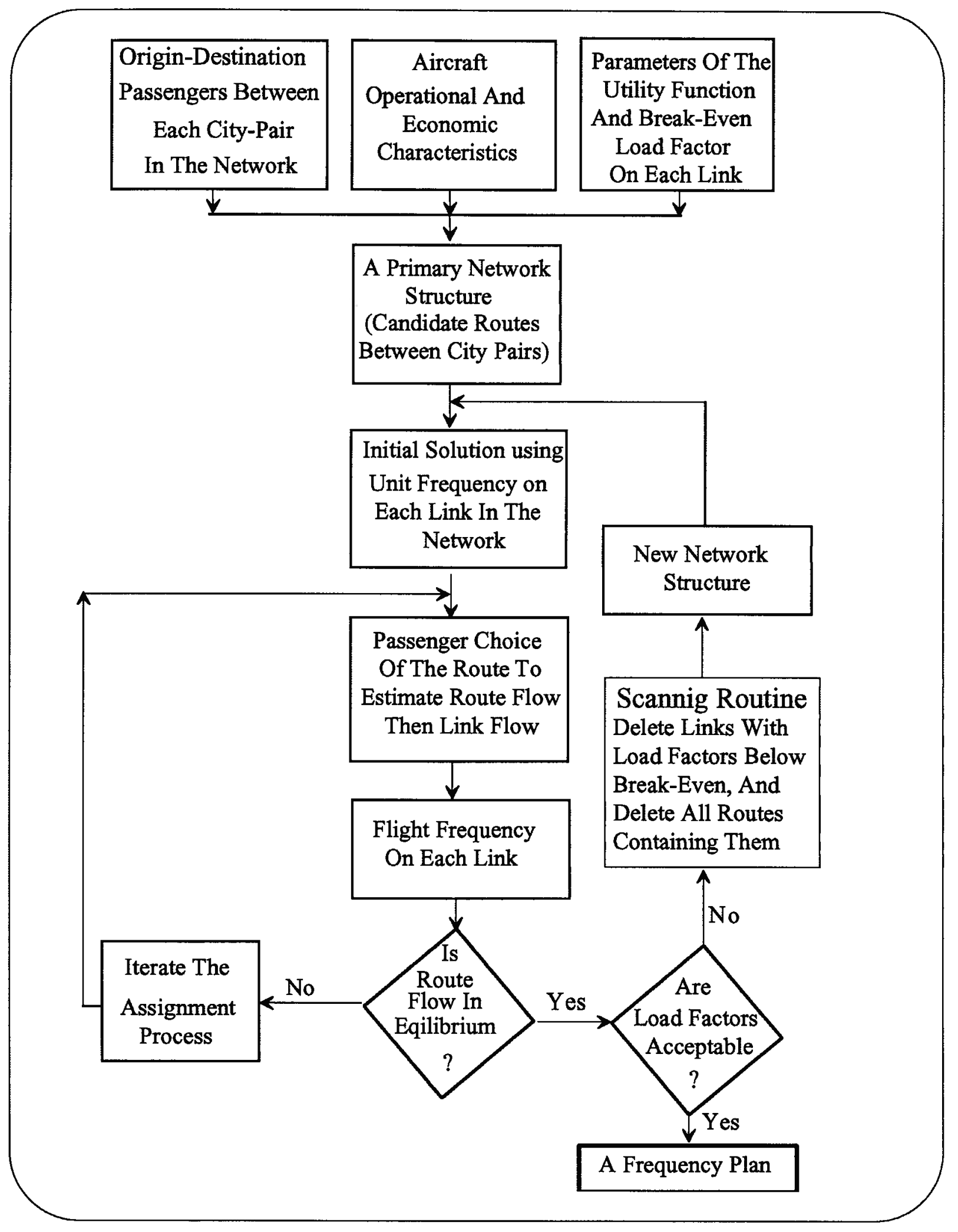

Figure 2.2: Flowchart of The Frequency Planning Model 
Another set of constraints is used to ensure the structural relationship between link and route frequencies. The frequency of service on a given route must be the minimum of frequencies on all links contained in that route

$$
F_{r}=\operatorname{Min}_{i j \in L_{r}}\left\{F_{i j}\right\}
$$

Where :

$F_{r} \quad=$ Service frequency on route $r$.

$L_{r} \quad=$ Set of links contained in route $r$.

$F_{i j}=$ Service frequency on link ij .

To run the model, unit frequencies are initially assigned to all available routes in the network. The traffic is then distributed to these routes (and then to the links) on the bases of a route choice model. The route choice model is built on the concept that traffic demand for flying a particular route is dependent on the different levels of service attributes of that route (i.e. travel time, frequency of flights, travel cost, number of transfers, etc.). The traffic between any O-D pair will be distributed among the different routes joining that pair according to the different service levels offered on these routes. This traffic distribution process is accomplished by using the multinomial logit model which relates the flows on different routes joining any city-pair as follows in equation (5):

$$
n_{r}=\frac{e^{v(r)}}{\sum_{\substack{k \in K_{o d} \\ e^{V(K)}}}}
$$




$$
n_{i j}=\sum_{r \in R_{i j}} n_{r}
$$

where:

$n_{r} \quad=$ Passenger flow on the $r$ th route connecting the O-D pair

$K_{o d}=$ set of all routes connecting the O-D pair

$R_{i j}=$ set of all routes containing link $\mathrm{ij}$

$V(r)=$ utility function for the $r$ th route

$n_{i j}=$ traffic on link $\mathrm{ij}$

Equation (6) is used to ensure that the flow on any link is equal to the sum of all flows on all routes containing that link. The utility function $V(r)$ is used as a measure of the service attributes of the route and it is assumed to be linear. Service attributes considered in this model include flight frequency, travel time, and air fare. For example, If $F_{r}$ is the service frequency on route $\mathrm{r}, T_{r}$ is the travel time on route $\mathrm{r}$, and $f_{r}$ is the fare on route $\mathrm{r}$, then the utility function $V(\mathrm{r})$ can be formulated as:

$$
V(r)=\alpha F_{r}+\beta T_{r}+\phi f_{r}+\ldots \ldots+\varepsilon
$$

where $\alpha, \beta, \phi, \ldots$. , are the attribute coefficients in the utility function and $\varepsilon$ is the error term of estimation. A characteristic of the logit model is worthy of noting here is that choice elasticity is not constant but is directly proportional to the value of attributes in the utility function (e.g. flight frequency) and inversely proportional to the probability of choice. This implies that the more likely a particular choice is, the less sensitive it is to changes in the value of the utility 
function (e.g. diminishing returns of choice with respect to increased frequency). The inclusion of both time and frequency in the utility equation (7) will then guarantee the convergence of the traffic assignment process.

According to the flowchart, new link frequencies are then calculated to satisfy the load factor constraints. The following set of equations are used to ensure that links with unprofitable flights are dropped out from the initial route network. The flight frequency $F_{i j}$ on link $\mathrm{i} j$ is expressed as:

$$
\left.\begin{array}{l}
F_{i j}=\left\{\begin{array}{cc}
\operatorname{Int}\left(\frac{n_{i j}}{\lambda_{i j}^{b}}\right) & \text { if } \frac{n_{i j}}{\lambda_{i j}^{b}} \geq \operatorname{Int}\left(\frac{n_{i j}}{\lambda_{i j}^{b}}\right) \\
\operatorname{Int}\left(\frac{n_{i j}}{\lambda_{i j}^{b}}\right) & \text { if } \frac{n_{i j}}{\lambda_{i j}^{b}}<\operatorname{Int}\left(\frac{n_{i j}}{\lambda_{i j}^{b}}\right) \\
0 \quad \text { if } n_{i j}<\lambda_{i j}^{b} s
\end{array}\right.
\end{array}\right\}
$$

where:

$n_{i j}=$ initial number of passengers on link $\mathrm{ij}$.

$n_{i j}^{\prime}=$ actual number of passengers on link ij.

$\lambda_{i j}=$ actual load factor on link $\mathrm{ij}$ 
$\lambda^{b}{ }_{i j}=$ break-even load factor on link ij.

$S \quad=$ aircraft capacity in terms of number of seats

According to Ghobrial etal, equations ( 8 a) and ( 8 b) ensure that an airline operates at least at its break-even load factor given indivisibility of flights. Equation $(8 \mathrm{c})$ guarantees that any link is deleted if its traffic does not justify profitable operation of a single flight when its resulting load factor is below the break-even level. This is the main reason for airline hubbing where low traffic density markets are served through a hub airport ${ }^{24}$ to consolidate the traffic demand and to increase the load factor. Because the indivisibility of flights, the frequencies obtained from equations ( 8 a) and ( 8 b) may result in load factors exceeding $100 \%$. This can take place when serving thin traffic market where providing two flights can result in a load factor below the break-even level. In such case, the airline will then have to operate only one full flight carrying $n_{i j}^{\prime}$ passengers. In this case however, the airline will lose $\left(n_{i j}-n_{i j}^{\prime}\right)$ unaccommodated passengers to its competitors. This condition is accounted for in equations (9 a) and (9 b).

Finally, The flowchart indicates that, the O-D demands are re allocated to different routes to account for the effects of the new frequencies on the route choice. The process is repeated until network equilibrium is reached when no change in both link frequencies and passengers flow take place. At this stage the scanning routine is used to delete all links with load factors below the break-even level. Consequently, all routes containing these links will also be deleted. According to Ghobrial etal, this entire process is iteratively repeated until network equilibrium is reached and the resulting load factors on all links are economically feasible. 


\subsubsection{Discussion Of The Model}

In a competitive market with a high level of demand, this model is a good tool for determining the optimum flight frequency plan for a given set of candidate routes, and as a consequence it will yield an optimum shape or configuration of the network. This can be accomplished by deleting links and routes from the initial set that do not meet the load factor constraints and keeping those that do. However, it can not be used as a stand alone model to provide an optimum frequency plan when the demand is sparse and airlines competition does not exist. This is because of the fact that the model was built mainly on the concept that frequency competition between airlines pushes them to operate at their break-even load factor. Hence, the main objective of the model is to formulate a frequency plan that will allow the airline to operate only on those routes that meet its break-even load factor constraints given the indivisibility of flights. The model does not consolidate the demand and it is not intended to do so. As will be shown later, when demand is sparse a more appropriate objective for the airline would be the minimisation of its operating cost by consolidating the demand and operating through a hub airport. In many situations as it will be seen in the case study later on, airlines are subsidized to provide air transport as a social service when operating uneconomical flights due to the lack of demand. In such situations, the airline will operate on some routes that will not meet its break-even load factor constraints. In such cases the frequency planning model can be used as a second step to provide a flight frequency plan for a pre optimized set of routes that consolidate the demand. 


\subsubsection{The Hierarchical-Hub (Hierhub) Model For Airline Networks}

Y.H. Chou ${ }^{25}$ developed the hierhub model based on the hierarchical structure of the hub and spoke network which is shown in Figure 2. 3 - D. Four basic patterns of hub-and-spoke networks are shown in Figure 2.3. The names clearly indicate the attributes as : the single-hub, the double-hub, and the multiple-hub. Node 5 in Figure 2. 3 - A is the only hub that acts as a single transfer location connecting all the other nodes in the network. In the double-hub pattern nodes 4 and 5 serve as the transfer locations through which all flights have to go. In single and double-hubs the number of links is minimal and it is equal to $\mathrm{N}-1$ where $\mathrm{N}$ is the number of nodes in the network. This will imply the existence of only a single path between any two nodes which will basically eliminates the need for any trip assignment process since all trips will be assigned to that path. Consequently, traffic demands will be consolidated at the hub locations. In multiple-hub networks however, as hubs are all directly connected, the number of links is larger than that for a minimally connected network. Therefore, multiple paths will exist and provide greater flexibility in routing, resulting in more convenience to passengers. 


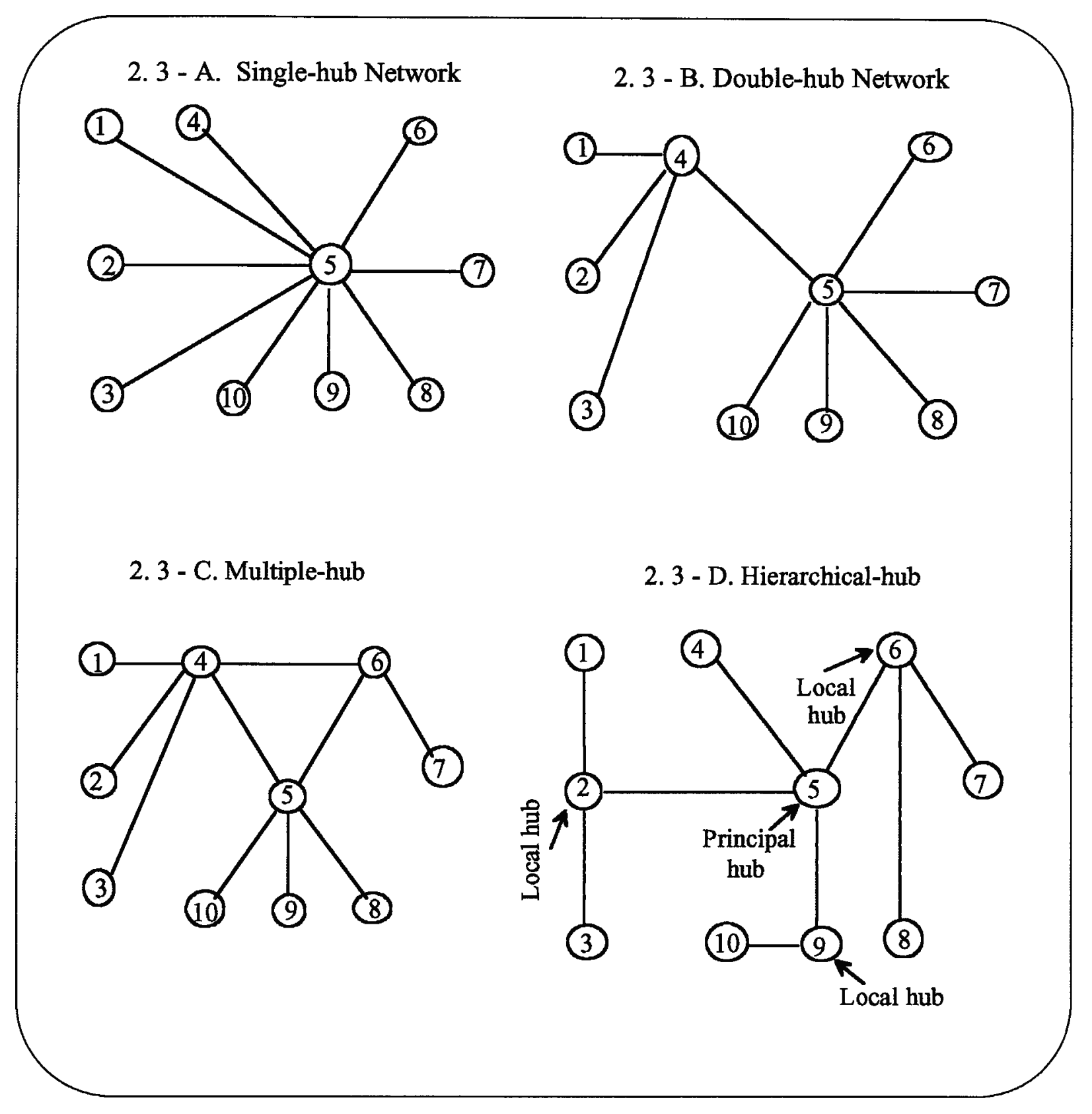

Figure 2.3 Patterns of hub-and-spoke networks

When sufficient air travel demand exist, multi-hub networks are in general more realistic because major carriers operate on networks with multiple paths. The provision of multiple paths in a network is the result of a balance between the savings in operational costs 
and the increased revenues from higher passenger patronage. In a hierarchical-hub networks, hubs may or may not be directly connected. The number of links is minimum, and there is only one possible path for each trip. Consequently, once the hubs are identified, links can be established and flows are assigned accordingly eliminating the need for a separate trip assignment process. As can be seen in Figure $2.3-\mathrm{D}$, in this type of network pattern, a node may be the principal hub, a local hub, or a spoke depending on siting and trip assignment. The total number of hubs is determined by the structure of the optimal hierarchical network. All the nodes that provide any degree of switching are considered hubs.

To summarize, the hierarchical-hub network may be considered as an early stage version of the multi-hub network. At a later stage, as traffic demand increase and the need for multiple paths emerges, a carrier may increase links and upgrade the network into a multi-hub system. This makes the hierarchical-hub an intermediate network between the double-hub and the multi-hub systems. In addition, both the single-hub and the double-hub networks can be considered as special cases of the hierarchical-hub network.

According to hierarchical-hub model the following exogenous variables are required for modeling Hierhub networks:

$N=$ The number of nodes in the network.

$O_{i}=$ The traffic flow originating from node $\mathrm{i}$.

$D_{j}=$ The traffic flow destinated for node $\mathrm{j}$.

$W_{i j}=$ The traffic volume from node $\mathrm{i}$ to node $\mathrm{j}$.

$C_{i j}=$ The unit transport cost between node $\mathrm{i}$ and node $\mathrm{j}$. 
The structure of a hierarchical hub-and-spoke network is expressed mathematically by an $\mathrm{N} \mathrm{X} \mathrm{N} \mathrm{connectivity} \mathrm{matrix}{ }^{26}$. The first degree connectivity matrix shows the presence or absence of a direct link between each origin-destination (O-D) nodes in the network. If a direct link exist, its representing element in the matrix will be assigned a value of 1 ; otherwise the element will be assigned a value of zero. Similarly, the second degree connectivity matrix shows the indirect connectivity between each O-D pair utilizing the combination of two direct links.

For developing the hierhub model, connectivity matrices are modified into the $E$ matrices. These $E$ matrices are developed for a minimally connected network. A network is considered minimally connected if all of its nodes are connected by $N-1$ links. The first degree $E$ matrix, $E^{1}$, is similar to the first degree connectivity matrix except that the $E^{1}$ matrix must be minimally connected. $E_{i j}{ }^{1}$ is the $i$ th - row and $j$ th - column element. It is equal to one if there is a direct link between the corresponding nodes; otherwise it is equal to zero. Similarly, The second and the third degree $E$ matrices, $E^{2}, E^{3}$, are constructed. The degree of $E$ matrices ranges from one to the diameter of the tree which is defined as the minimal number of steps or links between the most distant node pair in the network. An important distinction between $E$ matrices and connectivity matrices is that for each O-D pair only the shortest path is recorded in the $E$ matrices. And if a path appears in a lower degree $E$ matrix for any O-D pair, the same O-D pair in all higher degree matrices will be zero. Mathematically, the $E$ matrices are specified by Chou as follows:

$$
E_{i j}^{k} \in(0,1) \quad \forall i, j, k
$$




$$
\begin{aligned}
& E^{k}=0 \quad \forall i, k \\
& \sum_{k}^{i i} E^{k}=1 \\
& \sum_{i j}^{k}=E^{k} \\
& \sum_{i j}^{j} \sum_{i j}^{1}=2(N-1) \\
& \sum_{j} E_{i j}^{1} \geq 1 \\
& \sum_{j} E_{i j}^{1} \leq N-1
\end{aligned}
$$

The above equations were developed to satisfy the following constraints: Equation (1) requires that each element in the $E$ matrix is either 1 or 0 depending on the presence or absence of connectivity ( link ) for the corresponding degree k. Equation (2) requires that all path from one node to itself are non-existent. Equation (3) indicates that only the shortest path for each O-D is considered. Equation (4) states that each O-D pair has symmetrical paths. Equation (5) ensures the matrix to be minimally connected. Equation (6) ensures that each node to be directly connected with at least one other node. Equation (7) requires every node to be directly connected to no more than $N-1$ other nodes to guarantee no circuit exists in the network.

For illustrative purposes, Figure 2.4 shows an example of a 6-node network which is minimally connected. The largest degree of the $E$ matrices is four since the diameter of the network is equal to four. This is because the longest path which is between nodes 4 and 6 in the network takes four steps; 4 to 3,3 to 2,2 to 1 , and 1 to 6 . 


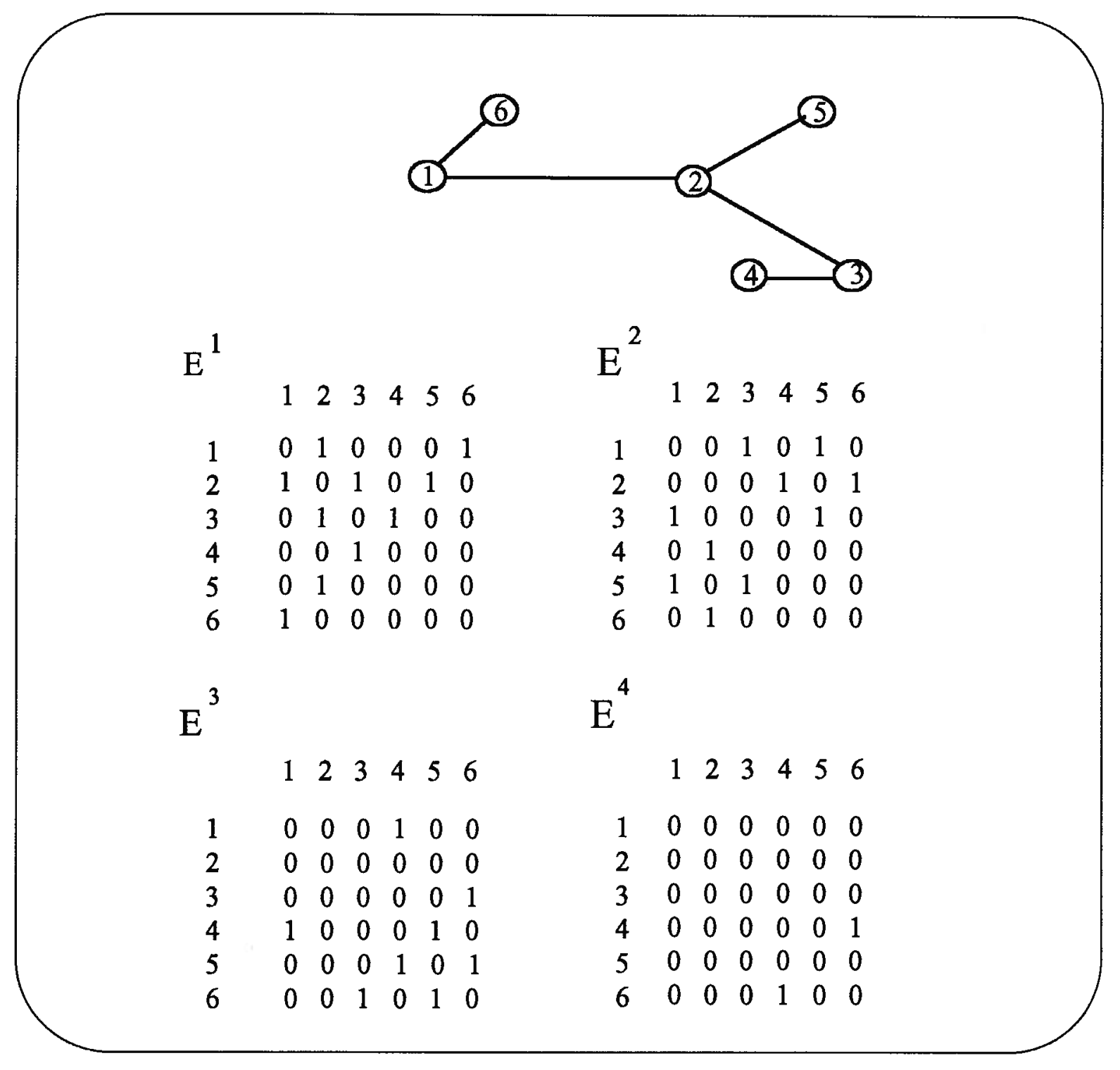

Figure 2. 4 Example Of The E matrices Of A 6-Node Network

According to Chou, the first step of the hierhub modeling is to build a minimal spanning tree for each node as the root of the tree and the node is denoted as $r$. For each tree at least an $E^{1}$ matrix is obtained and a new transport cost, $C_{\mathrm{ij}}^{\mathrm{r}}$, can be determined which is defined as the total cost of travel from node $i$ to node $j$ in the tree such that: 


$$
\begin{aligned}
C_{i j}^{r}= & C_{i j} E_{i j}^{1} \\
& +\sum_{m}\left(C_{i m}+C_{m j}\right) E_{i m}^{1} E_{m j}^{1} E_{i j}^{2} \\
& +\sum_{m} \sum_{p}\left(C_{i m}+C_{m p}+C_{p j}\right) E_{i m}^{1} E_{m p}^{1} E_{p j}^{1} E_{i p}^{2} E_{m j}^{2} E_{i j}^{3} \\
& +\ldots \ldots \ldots . .+\sum_{m} \ldots \ldots \ldots \sum_{q}\left(C_{i m}+\ldots \ldots \ldots+C_{q j}\right) E_{i m}^{1} \ldots \ldots \ldots . . E_{i j}^{d}
\end{aligned}
$$

where $d$ is the diameter of the tree.

The number of elements in the above equation will be equal to the diameter of the tree. The first element is composed of direct links. The second element consists of two steps indirect paths, i.e., when transfer through an intermediate node is necessary to move from origin to destination. Similarly, the third element consists of three steps indirect paths, and so forth. It should be noted here that, for each $\mathrm{i}-\mathrm{j}$ pair there is one meaningful element only. For example, in the rooted spanning tree of figure 2. 4, between nodes 1 and 5 only $E^{2}{ }_{15}$ equals one while all the other $E_{15 \mathrm{~s}}^{\mathrm{k}}$ are zero. Accordingly, the $C_{\mathrm{ij}}^{\mathrm{r}}$ defines the actual transport cost for the two steps indirect path between nodes 1 and 5 .

The $C_{\mathrm{ij}}^{\mathrm{r}}$ cost (r-cost) can be greater than, equal to, or less than the given transport cost, $C_{i j}$, depending on the structure of the tree. It can be greater than $C_{i j}$ if the direct link of minimal cost between nodes $i$ and $j$ is not included in the tree. In this case the new cost is higher because an indirect path of higher cost must be used in connecting these nodes. The r-cost is equal to the transport cost either when the direct link is included in the tree or when the total transport cost of the indirect path is identical to $C_{i j}$. The r-cost can be less than $C_{i j}$ when there exists an indirect path of which the total transport cost is less than that of the direct link. This situation 
can take place due to the scale effects associated with larger traffic volume which can result as the traffic gets consolidated along the indirect path.

After determining the $C_{\mathrm{ij}}^{\mathrm{r}}$ cost for each O-D of each tree rooted at each node, the model searches for the system which minimizes the total transport cost while satisfying the flow constraints. This can be mathematically formulated as follows:

$$
\operatorname{Min} Z=\sum \sum_{i j} C_{i j}^{r} W_{i j} \quad \forall r
$$

subject to:

$$
\begin{aligned}
& \sum_{j} W_{i j}=O_{i} \\
& \sum_{i} W_{i j}=D_{j}
\end{aligned}
$$

The final result of the model includes an optimum spanning tree which connects all the nodes in the network, the traffic volume assigned to each link, and the traffic volume going through each node. In the optimal system, any node having traffic volume larger than its O-D volume is a hub because it handles connecting traffic. Otherwise it is a spoke. The hierarchical structure can be identified from both the linkages of the spanning tree and the assigned traffic volumes.

\subsubsection{Discussion Of The Model}

One important feature of this model is its ability to endogenously derive the optimal number of hubs and also identify their locations in the network. Models that require this number 
to be provided arbitrarily as an exogenous variable have some difficulty in justifying that number. In order to accept an arbitrary number, one must solve the problem for different numbers of hubs and compare the solutions. Such comparative process can be time consuming and costly. However, this feature might not be desirable in all situations. For example, in some occasions a carrier might decide for one reason or another to establish a network with a certain number of hub facilities. In other occasions, some airports can not be developed as hubs either due to their lack of capacity or due to some other reasons. At the same time some other airports will be more suitable for that purpose but this model does not have any control mechanism that will help in dealing with this problem. Also, the model does not allow its user to add or delete any specific link in the network. Consequently, travelling between two non-hub airports might require two or even more transfers resulting in longer travel times. This is because the model was built to optimize the network for the carrier purposes and completely ignored the traveller.

\subsubsection{Other Work}

Todoroki, Hanzawa, and Fukuda $^{27}$ proposed an integer programming model that identifies the optimum location(s) of hub airport(s) among all airports in the network. The model basically assumes that all flights must go through a hub airport. The objective of the optimization is the minimisation of the total trip-distance. For a single-hub air network the objective function is:

$$
\begin{gathered}
\operatorname{Min}_{H}\left\{\sum_{i} O_{i} d\left(p_{i}, H\right)+\sum_{j} D_{j} d\left(H, p_{j}\right)\right\} \\
O_{i}=\sum_{j} W_{i j} ; \quad D_{j}=\sum_{i} W_{i j}
\end{gathered}
$$


where:

$W_{\mathrm{ij}} \quad=$ the amount of trips originated at airport $\mathrm{i}$ and terminated at airport $\mathrm{j}$.

$H \quad=$ the serial number of an airport which is chosen as the hub.

$p_{i} \quad=$ the serial number of an airport $\mathrm{i}$.

$d(\mathrm{x}, \mathrm{y})=$ the distance between airport $\mathrm{x}$ and airport $\mathrm{y}$.

In the case of a multiple-hub network, the model assumes that each local airport is served by only the nearest hub airport. Also two flight patterns exist, one with flight path that goes through one hub only and the other with flight path that goes through two hub airports. In this case the model is formulated as follows:

$$
\operatorname{Min} \sum_{i} \sum_{j} W_{i j}\left\{d\left(p_{i}, H_{1}\right)+d\left(H_{1}, H_{2}\right)+d\left(H_{2}, p_{j}\right)\right\}
$$

For this model to work the number of hub airports in the network has to be chosen arbitrarily by the user as an exogenous variable and the earlier mentioned comparative approach of different numbers must be taken to determine the optimal number.

\subsubsection{Summary}

To summarize, It is safe to conclude that prior to the airline deregulation act of 1978 in the U.S., the majority of the academic work has concentrated on developing models that provide optimal solutions for scheduling flights over given sets of pre determined routes. The 
main concern of those models was to make the existing routes more profitable to operate. This was the case because routes were dictated by the government and the airlines did not have the freedom to alter them. For example, the previously discussed models that were developed in that time were created to either produce airline schedules that minimize the schedule delay time of the passengers, or to provide the optimal frequency of flights that will minimize the operating cost of the airlines on the given routes. After the deregulation, models were developed to provide optimal network configuration, optimal frequency of service, and optimal hub locations. It is important to remember that these models, as with any mathematical models, can not represent the real world in perfect detail. Also, there is no specific criteria as to what detail is sufficient enough. Inescapably, a model will not meet someone's notions of what is the right level of details because each model has a limited scope and certain objectives to satisfy. However, any model can not generally be judged right or wrong. Every model has a certain value, which depends on how important the problem being modelled is, and how much insight the model provides into the problem. Finally, dealing with networks that serve sparse demands is unique because such networks have not been specifically addressed in the published literature. 


\section{METHODOLOGY}

This chapter outlines the methodology to develop the routing algorithms. Also a short review of linear and integer programming techniques will be given. A presentation of the mechanics, the assumptions, and the implications of the assumptions involved in these two techniques will be discussed to provide a better understanding of the methodology. The routing algorithms and the necessary data elements required to optimize sparse networks will also be presented.

\subsubsection{Linear And Integer Programming}

According to $\mathrm{Wu}$ and Coppins ${ }^{28}$, linear programming is a mathematical technique that is normally used as a decision aid tool for selecting the best course of action ( the optimum alternative or solution ) from a given set of feasible alternatives. It is called linear because the relationships between the variables involved are linear.

A typical linear programming problem can be presented as follows: Optimize (either maximize or minimize) a dependent variable which is a linear function of some independent variables subject to a series of linear constraints involving the independent variables. The dependent variable is known as the "objective function" and usually involves economic concepts such as; profits, revenues, costs, sales, time, distance, etc. The objective function is an equation that defines the quantity to be optimized. The independent variables in the objective

function equation are called "the decision variables" because the decision maker must determine 
the values of the variables that optimize the objective in order to solve the problem. An optimal solution to any linear program includes a set of values for the decision variables or sets of values in some cases when the solution is not unique. It also includes the corresponding values of the objective function. A standard linear program can be written in symbols as follows:

$$
\begin{aligned}
& \text { Optimize } \quad f=c_{1} x_{1}+c_{2} x_{2}+\ldots \ldots . .+c_{n} x_{n} \quad \text { or } \quad \sum_{j=1}^{n} c_{j} x_{j} \\
& \text { Subject to } \quad a_{11} x_{1}+a_{12} x_{2}+\ldots \ldots .+a_{1 n} x_{n} \leq b_{1} \\
& a_{21} x_{1}+a_{22} x_{2}+\ldots \ldots .+a_{2 n} x_{n} \leq b_{2} \\
& a_{i 1} x_{1}+a_{i 2} x_{2}+\ldots \ldots+a_{i n} x_{n} \leq b_{i} \\
& a_{m 1} x_{1}+a_{m 2} x_{2}+\ldots .+a_{m n} x_{n} \leq b_{m} \\
& \sum_{i=1}^{n} a_{i j} x_{j} \leq b_{j} \quad i=1, \ldots . ., m \\
& x_{1}, x_{2}, \ldots \ldots . ., x_{n} \geq 0
\end{aligned}
$$

where

$x_{1}, x_{2}, \ldots \ldots ., x_{n}=$ decision variables

$$
f=\text { objective function }
$$

$c_{1}, c_{2}, \ldots \ldots . ., c_{n}=$ coefficients of decision variables in the objective function

$a_{i 1}, a_{i 2}, \ldots \ldots . ., a_{i n}=$ coefficients of decision variables in the ith constraint

$$
b_{i}=\text { constant (the right hand side of the ith constraint) }
$$

Since in practice linear programming problems normally deal with a very large number of variables and constraints ${ }^{29}$, they are mostly presented in vector and matrix notation. Thus:

$\operatorname{Max}$ or $\operatorname{Min} f=C X$ 
Subject to $\mathrm{AX} \leq$ or $\geq \mathrm{B}$

where $\mathrm{C}, \mathrm{X}$, and $\mathrm{B}$ are row and column vectors, and $\mathrm{A}$ is the matrix of $a_{i, j}$ parameters of the variables in the system of constraints.

\section{$\underline{3.1 .1 \text { Assumptions }}$}

According to De Neufville ${ }^{30}$, the power of linear programming is a direct result of the assumptions it makes about a problem. The main assumption of linear programming is that the objective function and all the constraints are linear. Also, the decision variables are assumed to be nonnegative and continuous in the case of linear programming. In the case of integer programming the decision variables are assumed to be nonnegative and integers.

Linearity. The idea of linearity here is more specific and limited than the general idea that the majority of people have about a linear equation. Generally, a linear equation is thought of as one with all variables contained in the equation are raised to the power of one.

Formally, a function $f(\boldsymbol{X})=f\left(X_{1}, \ldots \ldots, X_{n}\right)$ is linear if, for all variables $X_{i}$ and constants $\mathrm{S}_{i}$ :

$$
f\left(S_{1} X_{1}, \ldots \ldots \ldots S_{n} X_{n}\right)=S_{1} f\left(X_{1}\right)+\ldots \ldots \ldots+S_{n} f\left(X_{n}\right)
$$

This definition can be split into two equivalent statements:

1. Constant returns. A linear function must have constant returns or economics of scale. This implies that $\quad f(S \boldsymbol{X})=S f(\boldsymbol{X})$ 
which really means that multiplying every decision variable by a common factor $S$ leads to an $S$-fold change in the objective function or constraint.

2. Additivity. A linear function must be additive which means that the value of the function with all $X_{i}$ simultaneously is equal to the sum of the values of that function with each $X_{i}$ by itself:

$$
f\left(S_{1} X_{1}, \ldots \ldots \ldots S_{n} X_{n}\right)=S_{1} f\left(X_{1}\right)+\ldots \ldots \ldots+S_{n} f\left(X_{n}\right)
$$

this implies that the contribution of each $X_{i}$ to $f(X)$ should not depend on the presence or absence of any others.

Continuity. As mentioned previously, linear programming assumes that the decision variables $X_{i}$ are both continuous and nonnegative. The continuity assumption means, as a practical matter, that the physical realities do not restrict them to integer or discrete values. This restriction can be seen as a difficulty since many systems do indeed consist of design elements that can only be integer or discrete. For instance, the number of aircraft in a fleet must be an integer, as must be the number of warehouses in a distribution system. In practice however, the assumption of continuity dose not constitute a major difficulty. This assumption can be dealt with in two ways. First, we can simply assume that integer variables are continuous and round off our results. In many situations this will be satisfactory if the possible error of a few percent is within the accuracy with which we can formulate any real problem. Second, in some situations, some people might argue that the rounding off approach might violate some of the constraints or it might produce a nonoptimal solution. In such situations, integer programming which offers a way to formulate linear programs that can cope with integer values can be used. 
Nonnegativity. This assumption which restricts all of the decision variables to nonnegative values is merely a technicality imposed by the way a linear program finds the optimal solution. In practice, this requirement does not really restrict us since we can always define variables so that they are not negative. Consequently, this assumption just requires that we be careful in the way we formulate a linear program.

\subsubsection{Graphical Presentation Of Linear Programming}

As long as the number of decision variables is 2 , linear programs can be analysed and solved graphically. Consequently, a graphical approach is not practical since most real world problems involves a large number of decision variables. However, a graphical presentation here for a hypothetical problem as seen in figure 3. 1 will provide valuable insight into the relationships and solution procedure of linear programming in general.

For illustration purposes, the following problem is to be analysed :

Maximize

$$
\begin{gathered}
\mathrm{f}=12 \mathrm{X}_{1}+8 \mathrm{X}_{2} \\
6 \mathrm{X}_{1}+2 \mathrm{X}_{2} \leq 120 \\
3 \mathrm{X}_{1}+4 \mathrm{X}_{2} \leq 120 \\
\mathrm{X}_{1}, \mathrm{X}_{2} \geq 0
\end{gathered}
$$

Subject to

where:

the objective function $f\left(X_{1}, X_{2}\right)$ can be thought of as the profit from selling two products. The coefficients in the function represent the per unit profit from each product. $\mathrm{X}_{1}, \mathrm{X}_{2}$ are the two products. The right hand side of the two constraints represent the amount of two resources available for manufacturing the two products, while the coefficients in the constraints represent 
the amount required per each unit of production. The problem here is to find the most profitable mix of the two products.

The above problem is presented graphically in figure 3.1 by letting the horizontal axis represent $\left(X_{1}\right)$ and the vertical axis represent $\left(X_{2}\right)$. The nonnegativity constraints mean that only the first quadrant is meaningful. The two constraints are plotted by finding their intercepts on each axis and lines were connected through them. Any feasible solution or production mix of the two products must satisfy the two constraints simultaneously. Therefore, the set of feasible solutions is the intersection of the sets of points which satisfy each constraint. This set is shown in figure 3. 1 by the shaded area in the figure. To find the optimal solution, the objective function (f) must be considered. But since we cannot graph (f) per se since it does not have a fixed value, its " iso line" for a particular value can be graphed. In this example, its iso line for $f=120$ is drawn. "Iso " means the " same ", in this case each point on the line $f=120$ is a combination of the two products that will yield a profit of 120 . Since this problem requires the maximization of the objective function, this means that we must construct the " iso line " which has the largest value of the objective function and still passes through the set of feasible solutions. Alternatively, the process of finding the optimal solution can be envisioned as drawing an iso line for the objective function through the set of feasible solution and then pushing it as far as it can go while still passing through the set. In this example, the optimal solution occurs at point (A), which is the only point in the feasible region through which the maximum iso line ( $\left.\mathrm{f}^{*}\right)$ passes. 


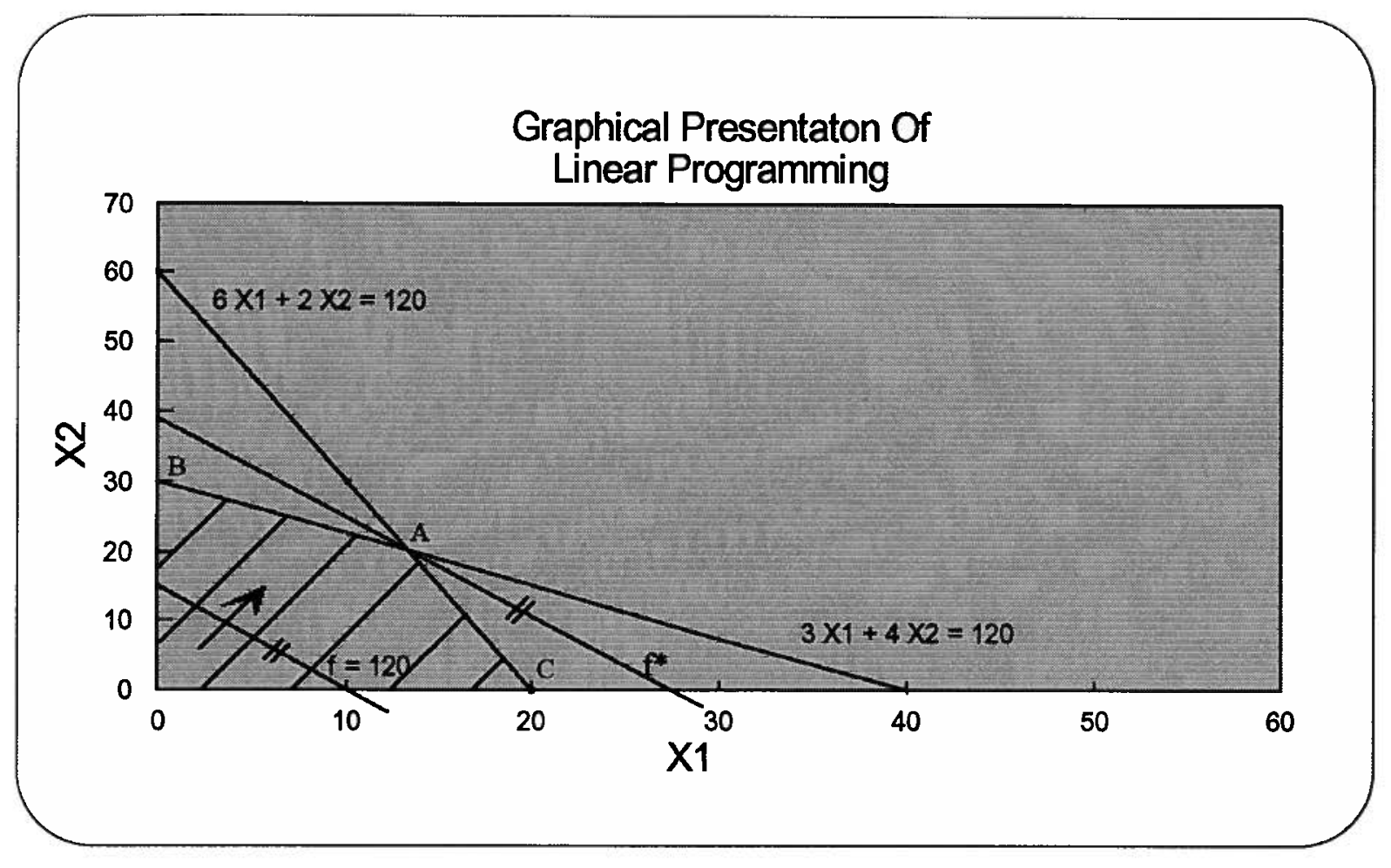

Figure 3. 1 Graphical Presentation Of Linear Programming

As mentioned above, the optimal solution to the given example occurred at point (A), which is a corner point in the set of feasible solutions. This did not happen by a chance. It can be shown by the solution concept discussion which will be presented below, that the solution to linear programming problem always can be found at a corner point or points of the set of feasible solutions.

\subsubsection{Solution Concept}

The solution to any linear programming problem no matter how complex or sophisticated the problem is, is entirely based on two important consequences of the assumption 
of linearity of the objective function and constraints. In order to present the solution concept, it is first essential to present the effects of the linearity assumptions, which provide the basis for all linear programming procedures.

\section{Effects Of Linearity Assumptions.}

The linearity assumption has two effects, they are:

1. If it exist, the feasible region is convex.

2. The optimum solution is located at an edge of this region, particularly at one of its corners. These two facts greatly reduce the optimization procedure to a search through a limited set of well defined possible solution combinations located specifically on the edges of the feasible region, which is very small compared to the infinite number of solution possibilities contained in the entire feasible region.

To facilitate the following discussion of the effects of linearity assumption, it is important to define what is meant by a convex feasible region. Formally, a feasible region is convex if every straight line between any two points in the region lies entirely in the region. As it can be seen in figure 3.2, a convex feasible region is therefore one that has no reentrant boundaries, no edges that intrude or dent into its space. It is worth to mention that, the convexity of the region does not simply depend on the shape of its boundary. As it can be seen in the same figure, the same boundary can be associated with both convex and nonconvex regions. 


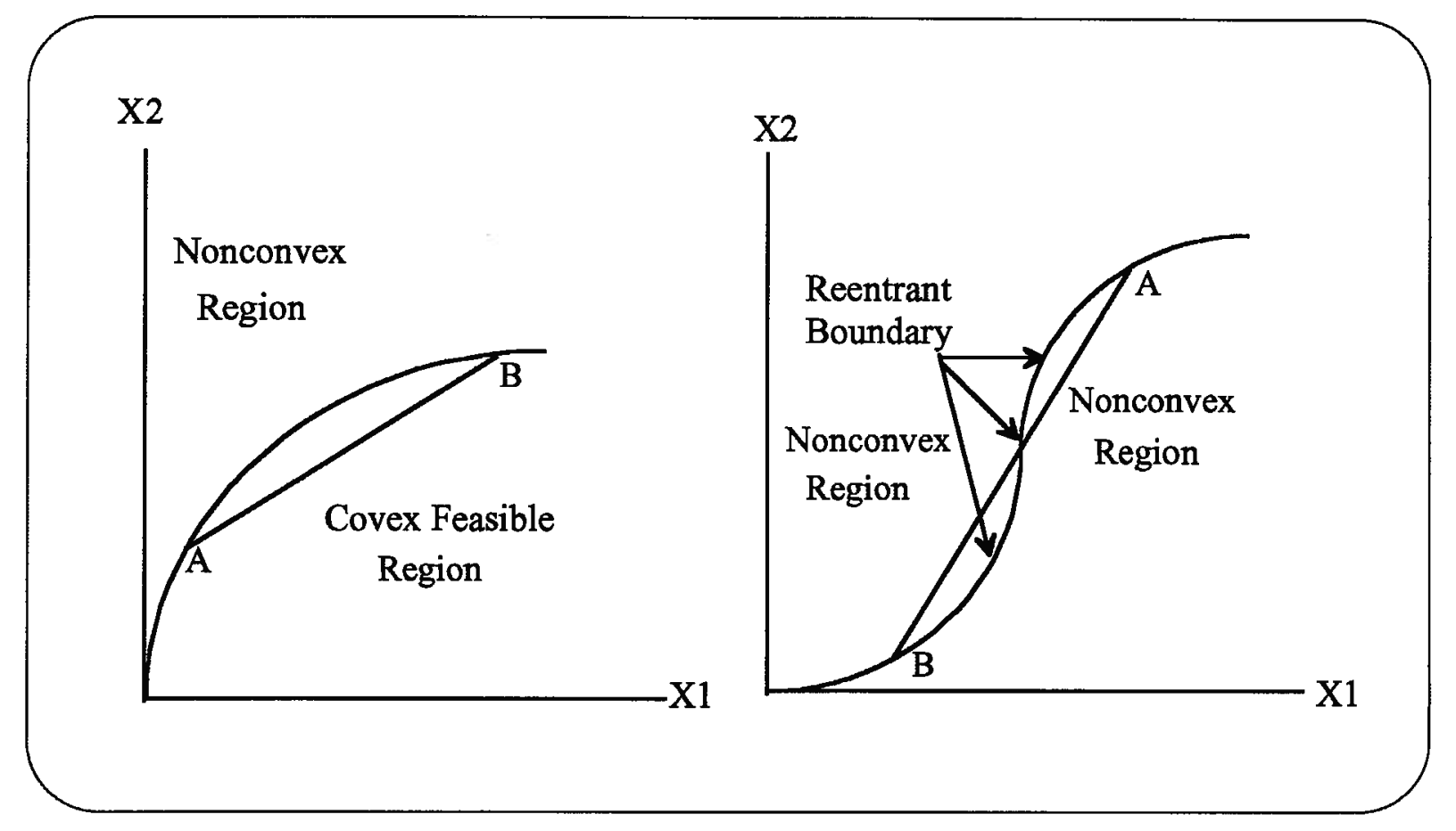

Figure 3. 2 Convex And Nonconvex Feasible Regions

To explain the two effects of linearity, it is helpful to visualize the feasible region to be consisting of two parts. As shown in figure 3.3, these two parts are:

1. The space defined by the constraints.

2. The volume defined by the relationship of the decision variables to the objective function.

If the feasible region defined by the linear constraints exist, it must be convex since any two points that satisfy any constraint must be on one side of that constraint inside the feasible region to satisfy all the other constraints and any line between them will be entirely in the feasible region. Therefore the operational definition of convexity is met. At this point it is worth mentioning that, a feasible region might not exist at all. This situation can take place if the constraints are contradictory and there is no possible set of decision variables that will 
satisfy all constraints simultaneously. In this case the solution is infeasible. A good example for this situation in the real world is the possibility of not being able to meet some environmental standard with the available technology or resources.

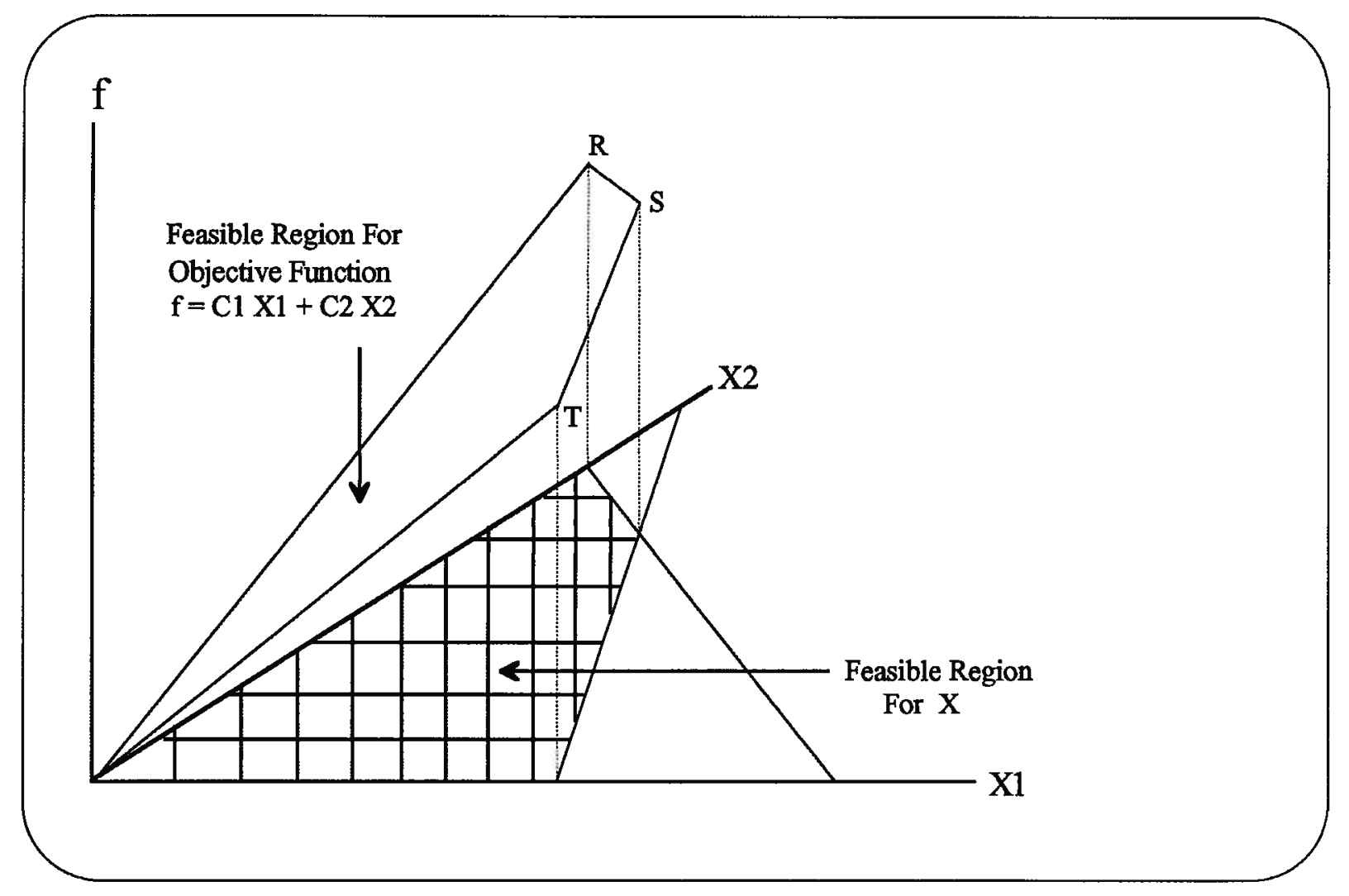

Figure 3. 3 The Two Convex Parts Of The Feasible Region

By the same argument as before, the volume defined by the objective function and constraints is also convex. And since the objective function is linear, it can be concluded that the rate of change from any point interior to the region in any direction is constant. Hence, there must be an edge that has a value of the objective function equal to or greater than any interior point in the region. Only one exception to this can occur if the problem is unbounded so that 
it is possible by the way the constraints are set to increase the objective function infinitely. Similarly, since the rate of change of the objective function a long every edge is constant, the value of the objective function must be equal to or greater at one of the corner points to that edge than anywhere in the middle of the edge. Therefore it can be concluded that the optimal solution must be located at a corner point. In the example of figure 3.3, when maximizing the objective function the solution must be at $\mathrm{R}, \mathrm{S}$, or $\mathrm{T}$.

It is possible to have multiple optimal solutions for a linear program. This situation can take place when the rate of change of the objective function along an edge is zero (The objective function and a constraint have the same slope). In such case, the entire edge, including its corner points, constitutes a set of optimal solutions.

Numerous computer programs are available for solving linear programming problems. They differ in their degree of sophistication and range from smaller routines that run on personal computers to very large programs that can deal with thousands of variables. To solve a linear programming problem, all programs are essentially developed to conduct an organized search for the solution through the corner points. The general procedure for finding the optimal solution can be described as follows:

1. Find a corner point as a feasible solution to all constraints.

2. Proceed sequentially to other corner points that provide better solutions to the problem.

3. The search should stop at the corner point from which no adjacent corner leads to a better value of the objective function. 
In practice, the different computer programs utilize different methods to execute the procedure outlined above. These methods will not be presented in this thesis since they are not the main concern.

\subsubsection{Integer Programming}

Integer programming is a variation of linear programming which forces the solution to be integer ${ }^{31}$. In many real world situations it is necessary for the decision variables to take on only integer values. For instance, the number of aircraft in a fleet must be an integer, as must be the number of warehouses in a distribution system. Also as it will be seen later in this thesis, any alternative route between to locations in a given transportation network can either take on the value of one or zero. One if the route should be included in the final optimal network or zero if it should not. Such situations can be dealt with in two ways. First, we can simply assume that integer variables are continuous and round off our results. In many situations this will be satisfactory if the possible error of a few percent is within the accuracy with which we can formulate any real problem. Second, in some situations, some people might argue that the rounding off approach might violate some of the constraints or it might produce a nonoptimal solution. In such situations, integer programming which offers a way to formulate linear programs that can cope with integer values can be used. For this thesis, it is satisfactory to consider integer programming as only a slight variation of linear programming. The problems in both techniques are set-up in the same manner and with the exception of the continuity assumption, all other assumptions are the same in both cases. 


\subsubsection{Formulation Of The Problem.}

Before introducing the formulation designed for the problem it is helpful to present some definitions that will be used in the remainder of the thesis. In this thesis, an air transport network consist of nodes, routes, and links. Nodes represent the airports in the network. Each node or airport is assigned a specific number. Routes represent the paths that flights are assigned to and follow between the origin and destination airports. Each route in this thesis can consist of one or two links. If a route contains one link only, then it is a direct route. If it contains two links, then it is a transfer route. Links represent direct connections between two airports. A single link can constitute a direct route.

When designing a transport network the interests of both the passenger and the airline operator must be considered. It is assumed that passengers are rational and they will try to minimize their travel time and the costs associated with it while maintaining a certain level of convenience. For example, a rational passenger will try to minimize the number of transfers and reduce travel circuity. An airline operator however will try to minimize his cost of operation. If that operator is monopolizing the transport market as is the case in our case study or in the majority of areas where demand is sparse and there is only one airline operator serving the market, then travel circuity and the number of transfers are not as important to the operator as his cost of operation. Evidently, a conflict of interest might exist between the operator and the passenger. Therefore, three routing algorithms will be formulated to produce three distinct designs of "efficient air transport networks" to satisfy all interests. The algorithms will be similar in all aspects except that their cost functions will be tailored to reflect the interests of the 
passenger, the operator, and the combined interests of the two together. The resulting network designs will provide valuable insight for the decision maker on how to best meet the expectations and the objectives of both the passenger and the operator. Any resulting design should not be considered or looked at in isolation as a final design by it self. The three designs must be evaluated together and used as a decision making tool that helps the decision maker. The objective functions and the constraints of the algorithms can take the general mathematical form presented below :

Minimize :

$$
\sum_{i=1}^{n} \sum_{\substack{j=1 \\ j \neq i}}^{n}\left(C_{i j} Y_{i j}+\sum_{\substack{m=1 \\ m \neq i \text { orj }}}^{n} C_{i m j} Y_{i m j}\right) \forall i, j, m \text { where } \operatorname{pax}_{i j}, \text { pax }_{i m}, \text { pax }_{m j}>0
$$

Subject to:

1. $Y_{i j}+\sum_{\substack{m=1 \\ m \neq i o r j}}^{n} Y_{i m j}=1 \quad \forall i \& j=1,2,3, \ldots . ., n ;$ and $i \neq j$

2. $Y_{i m}-Y_{i m j} \geq 0 \forall i, j, \& m=1,2,3, \ldots . . n$; and $i \neq j, i \neq m, j \neq m$

3. $Y_{m j}-Y_{i m j} \geq 0 \forall i, j, \& m=1,2,3, \ldots . . n$; and $i \neq j, i \neq m, j \neq m$

4. $Y_{i j} \& Y_{i m j} \geq 0 \quad \forall i, j, \& m=1,2,3, \ldots . . n$; and $i \neq j, i \neq m, j \neq m$

5. $Y_{i j}=1$ or $0 \quad$ all $i, j \in n$

The above costraints are only applicable where $\operatorname{Pax}_{i j}, \operatorname{Pax}_{i m}, \& P a x_{m j}>0$

Where:

$i=$ origin airport index.

$j \quad=$ destination airport index.

$m \quad=$ hub or transfer airport index where $\operatorname{Pax}_{i m} \& P a x_{m j} \neq 0$.

$n \quad=$ the total number of airports in the network.

$C_{i j}=$ the unit cost function of transport between airports i and $\mathrm{j}$; where $C=f(P a x, S, F C$, $B T, A D T, V T, N F, O P P)$.

$C_{i m j}=$ the unit cost function of transport between airports $\mathrm{i}$ and $\mathrm{j}$ through airport $\mathrm{m}$; where $C=f(P a x, S, F C, B T, C T, A D T, V T, N F, O P P)$. 
$Y_{i j} \quad=$ direct link between origin and destination airports. It is a decision variable with a value of either 1 or 0 .

$Y_{i m j}=\operatorname{transfer}$ link between $i$ and $j$ through $m$. It is a decision variable with a value of either 1 or 0.

Pax $=$ the number of passengers.

$S \quad=$ aircraft seating capacity.

$F C \quad=$ cost of one flight linking two airports.

$B T \quad=$ block time.

$C T=$ average flight connection time spent by passengers at a transfer airport $=1.5 \mathrm{hr}$.

$A D T=$ average schedule delay time.

$V T=$ value of time.

$N F \quad=$ minimum number of flights required to satisfy all demand $=P a x / S$.

$O P P \quad=$ operating period.

The objective function as presented above, will compare the unit costs of transport of the two routing strategies for each origin-destination pair in the network. The least costly strategy for any given pair will then be selected. The cost provided by this objective function is not an accounting cost, but it is a heuristic that provide sensible results in the cases examined. All airports in the network that has travel demand interaction with both the origin and destination airports are considered as potential transfer airports. The first constraint of the model is imposed to select only one routing strategy and one route for each origin destination pair in the network. This constraint in effect will constitutes a process similar to the "all or nothing" route assignment process of the urban transportation planning. The second and third constraints are imposed to only select transfer airports that has direct link with both the origin and destination airports in the final network design. The fourth constraint is the general none negativity constraint of linear programming. The fifth constraint is optional side constraint, any additional side constraint of the form $Y_{i j}=1$ would specify links (i,j) that are necessarily included in the final network's design. This type of constraint is necessary to model any proposed improvement in the network or to evaluate the development of a particular airport as a hub in the network. 


\subsubsection{Airline's Network Design}

The first network is designed to meet the operator objective by minimizing the airline's total operating cost. This can be achieved by consolidating the travel demands at some ideal hub or transfer locations which will be determined by the algorithm in accordance to the demand distribution throughout the network. Consolidating the travel demands will inevitably minimize the airline's operating unit cost of transporting passengers between the different demand locations. The unit cost of transporting passengers can be defined as the cost of transporting one passenger between the origin and destination airports. The following is the

mathematical formulation of the cost functions that will minimize the operator's cost of operations as can be used in the general algorithm as presented above:

$$
\begin{aligned}
& C_{i j}=F C_{i j} * N F_{i j} \div P a x_{i j} \\
& C_{i m j}=F C_{i m} * N F_{i m} \div\left(P a x_{i j}+P a x_{i m}\right)+F C_{m j} * N F_{m j} \div\left(P_{a x}+P_{i j} x_{m j}\right)
\end{aligned}
$$

Where:

$$
N F_{i j}=P a x_{i j} / S_{i j} ; N F_{i m}=\left(\operatorname{Pax}_{i j}+P a x_{i m}\right) / S_{i m} ; N F_{m j}=\left(\operatorname{Pax}_{i j}+P a x_{m j}\right) / S_{m j}
$$

The above cost functions are designed to calculate the airline's unit cost of transport for each origin destination pair that must be served according to the two routing strategies. Calculating the cost of direct routes is a straightforward task as indicated above. Calculating the cost of indirect routes for any given origin-destination (OD) pair however, must take account of the fact that passengers travelling between the (OD) pair can be flown on the same flights with 
passengers who are travelling between the origin and transfer airports, and those who are travelling between the transfer and destination airports.

The resulting savings in operational cost can either then be passed to the passengers in terms of reduced ticket prices, or they can be kept by the airline to improve its profitability. Passing the savings to passengers might stimulate more traffic which might further reduce the unit cost of operation however, this is a policy decision and calculating the effect of price reductions on traffic demand is not within in the scope of this thesis.

\subsubsection{Passenger's Network Design}

The second network is designed to minimize the passengers' cost of travel. This can be accomplished by selecting the routes that minimize both the travel time and the average schedule delay time required for passenger trips between the origins and destinations airports. It must be noted here that the actual cost of travel in term of ticket prices is not included in this design because it is directly related to the operational cost of the airline operator which is dealt with in the first design. Also, the third network design which will be presented later, will deal with this cost element to provide a balanced routing strategy that will provide a balance between the various costs involved. Travel time can be measured in many ways one of which is the door to door time. However, for reasons that will be explained later, travel time will be assumed equal to the "Block time" which is defined as the time span between the moment when the aircraft leaves the departing terminal and the moment when it stops at the arriving terminal ${ }^{32}$. Schedule delay time is defined as the difference in time between when a person would like or 
desire to make a trip and the time he or she is constrained to make the trip because of the availability of a flight according to the airline's schedule ${ }^{33}$. By assuming that the desired departure time is uniformly distributed over the entire period of flight operating hours per day, the average schedule delay time per day can be calculated as:

$$
A D T=\frac{\text { Length Of Operating Period }}{2 * \text { Flight Frequency }}=\frac{O P P}{2 * N F}
$$

This assumption may seems unreasonable in cases where the true distribution is not uniform. However, several studies including Oum's, and Tretheway's ${ }^{34}$ indicated that the assumption is adopted by the airline industry, therefore it will be satisfactory for the purposes of this thesis.

Minimizing the travel and schedule delay times is important because when a passenger wants to travel from one place to another he or she, must not only pay a price for it, but must also reckon with some other disadvantages including discomfort and must sacrifice a certain amount of time. While transportation costs and their prices are measured in terms of money, travel and schedule delay times are measured in time units. Consequently, these measures can not be added together because they are based on different criteria. This means that they must be grouped under a common denominator: either the common denominator of time or that of value. Traditionally, the common denominator of value has been used in most transportation studies to group the two elements. Thus, it is assumed that time equals money, and the time spent travelling or waiting to travel can be converted to its monetary equivalence by multiplying the time units by the value of travel time. The value of travel time is not an absolute value, this is so because the value depends not only on the trip circumstances, but also on the calculation method $^{35}$. 
Several Methods are available for estimating the value of travel time, For example, Bruzelius $^{36}$ presented the "Beesley method". The method is called after its originator M. E. Beesley. This method is built on the hypothesis that if a passenger can choose between two travel alternatives, e.g. modes, then the passenger will choose alternative 1 if :

$$
a_{01}+p_{1}+a q_{1}<a_{02}+p_{2}+a q_{2}
$$

where

$a_{01}=$ Fixed cost of alternative 1 .

$P_{1}=$ Price of alternative 1 .

$q_{I}=$ Travel time of alternative 1 .

$a=$ The value of time.

$a_{02}=$ Fixed cost of alternative 2 .

$P_{2}=$ Price of alternative 2 .

$q_{2}=$ Travel time of alternative 2 .

According to Bruzelius, the method assumes only one value of time exist, so that the passenger's willingness to pay to save time is the same for the two travel alternatives. To estimate this value of time, a sample of observations including data on the chosen alternative, prices, and time are recorded in a coordinate system as shown in Figure 3.4.

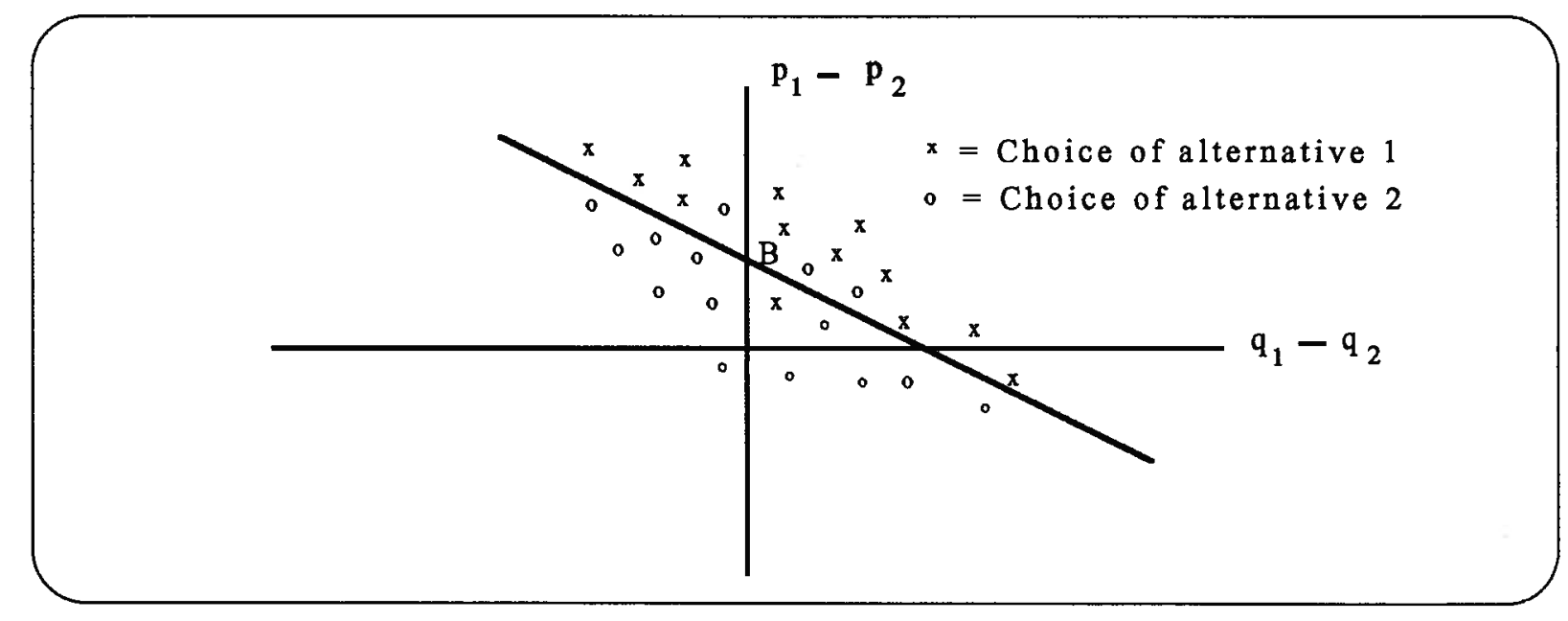

Figure 3.4 : Beesley's Method For Estimating The Value of Time 
A straight line is then drawn to separate the points representing the choices of the two alternatives. Bruzelius indicated that a perfect separation between the points can not be expected but an effort has to be made to make the number of points ending up on the wrong side of the line as small as possible. After the best line is located, the value of time is inferred from the slope of the line, and the difference in the fixed costs as represented by point $B$. The full details of this method is available in Beesley's paper which was published in $1965^{37}$.

Tarski $^{38}$ presented the "production-based method". The method is built on the hypothesis that shortening travel time of workers releases an additional amount of labour as a production factor that will increase the net and gross output of the society. Thus, a worker could add to the gross or net output during one hour of the time he would save on travelling. Hence, Tarski presented the following equation to estimate the value of time:

$$
V_{p}=\frac{P}{2000 p}
$$

where

$V_{p}=$ The value of one hour of travel time.

$P \quad=$ The annual amount of the gross or net product (in monetary units).

$p=$ The average number of persons employed in material production.

The 2000 is the average number of effective working hours in one year.

Harrison and Quarmby ${ }^{39}$ presented the "revealed preference method". In this method, a sample of travellers are asked to make a choice between 2 different travel modes with different travel times, costs, and other travel characteristics. Their choices are recorded and then used to calibrate the logit model of the Urban Transportation Planning process as presented below:

$$
L(x)=\sum a_{i} x_{i} \quad \text { and } \quad P_{1}(x)=\frac{e^{L(x)}}{1+e^{L(x)}}
$$


where

$x=a$ vector of values of relative characteristics of the two travel modes (time, cost, etc.).

$a_{i} \quad=$ a vector of parameters who's values are to be determined by the calibration process.

$L(x)=$ the relative disutility of the two modes.

$P_{l}(x)=$ the probability of a traveller or a group of travellers choosing mode 1 .

After calibrating the model, the value of time can then be estimated by dividing the time coefficients by the cost coefficients.

It it clear that there are various methods of calculating the value of travel time, but the most suitable method for the purposes of this thesis in terms of the data availability is the "cost saving method" presented by Harrison and Quarmby ${ }^{40}$. The method simply estimates the value of time by reference to the wage rates. Harrison and Quarmby indicated that this method is based on the marginal productivity theory of factor rewards which states "Employers hire labour until it is no longer worth their while to do so". Thus, the wage rate is a satisfactory measure of the value of production gained or lost by changes in labour force. And since reducing travel time of workers releases an additional amount of labour, then the wage rate is also a satisfactory measure of the value of time.

Due to the lack of data and studies on the value of travel time in Saudi Arabia, this thesis will utilize the cost saving method as mentioned above, and it will assume that the value of travel time is equal to the average hourly wage rate. Urban transportation studies traditionally use two thirds of the wage rate as an estimate of travel time. This traditional value will not be used in this study because air travellers usually earn more money than travellers using other 
modes of travel. Subsequently, air travellers time is more valuable ${ }^{41}$, and the full wage rate is more reasonable to use in this case. Also, the majority of domestic travellers in Saudi Arabia are business travellers ${ }^{42}$. Hence, any changes in travel times will greatly affect their work production, and the full wage rate will provide a better reflection of these changes. The value of the schedule delay time however, is assumed to be equal to half of the average hourly wage rate since it can be argued that this delay time can be used for accomplishing different productive tasks other than travelling. The average hourly wage rate in Saudi Arabia is estimated to be 28 Saudi Riyals (SR) per hour which is equivalent to 9 \$ Canadian. This estimate was made by pooling a number of people in different types of occupations, and by using the background knowledge of this author about the country. The following is the mathematical formulation of the cost functions that will minimize the passenger's cost of travel as can be used in the general algorithm as previously presented :

$$
\begin{aligned}
& C_{i j}=V T\left(B T_{i j}\right)+0.5 V T * A D T_{i j} \\
& C_{i m j}=V T\left(B T_{i m}+B T_{m j}+C T_{m j}\right)+0.5 V T * A D T_{x x} ; \text { where }\left\{\begin{array}{l}
x x=i m \text { if } N F_{i m}<N F_{m j} \\
x x=m j \text { if } N F_{i m} \geq N F_{m j}
\end{array}\right\}
\end{aligned}
$$

The above cost functions use the value of time to calculate the passenger's cost of travel time for each origin destination pair according to the two routing strategies. For this thesis, it is assumed that travel time is comprised of the following three components only: block and schedule delay times which were defined previously, and connection time which can be defined as the time spent in a transfer airport in order to get on a connecting flight to the final destination. The minimum connection time is assumed to be $1.5 \mathrm{hr}$. This assumption was made based on information provided by officials of the Saudi Arabian Airlines ${ }^{43}$. Only these 
time components were considered because they are the only time components that can be affected by the route choice. Calculating the cost of direct routes is a straightforward task. Calculating the cost of indirect routes however involves determining the connection time and the largest possible schedule delay time that a passenger has to experience because of the flight frequency at either the origin airport or the transfer airport.

\subsubsection{Combined Airline \& Passenger Network Design}

The third network is designed to minimize and provide a balance between both the operator and passengers' cost of travel. This can be accomplished by selecting the routes that minimize passengers' travel and schedule delay times with their associated costs, as will as the airlines' unit cost of operation between any given origin and destination airports. Striking a balance between the passenger and the operator costs of travel is important because as can be seen in Figure 3.5 some routes with their travel and schedule delay times might increase the passenger cost of travel because they either increase travel circuity or because they have low flight frequency. The same routes however might reduce the operator cost by consolidating the passenger demands and visa versa. Therefore, the following is the mathematical formulation of the cost functions that will provide a balanced routing strategy for both the passenger and the airline by minimizing their total cost of travel:

$$
\begin{aligned}
C_{i j}= & F C_{i j} * N F_{i j} \div P a x_{i j}+V T\left(B T_{i j}\right)+0.5 V T * A D T_{i j} \\
C_{i m j}= & F C_{i m} * N F_{i m} \div\left(\operatorname{Pax}_{i j}+P a x_{i m}\right)+F C_{m j} * N F_{m j} \div\left(\operatorname{Pax}_{i j}+P a x_{m j}\right) \\
& +V T\left(B T_{i m}+B T_{m j}+C T_{m j}\right)+0.5 V T * A D T_{x x} ; \text { where }\left\{\begin{array}{ll}
x x=i m & \text { if } N F_{i m}<N F_{m j} \\
x x=m j & \text { if } N F_{i m} \geq N F_{m j}
\end{array}\right\}
\end{aligned}
$$




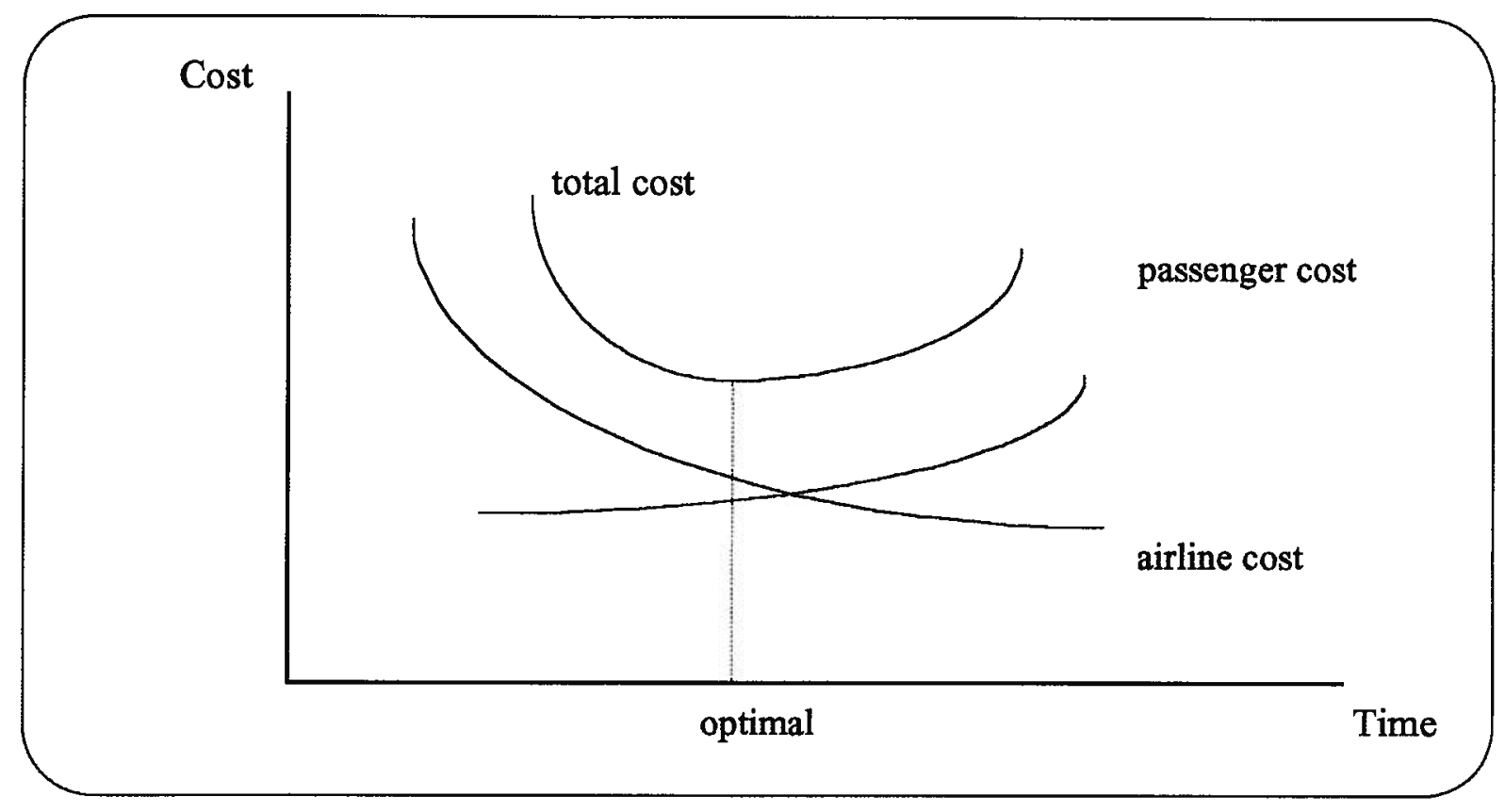

Figure 3.5 Finding The Optimal Economic Cost Of Travel

\subsubsection{Discussion And Validation Of The Formulations}

The formulations presented above seek to find efficient routing strategies for all demands between all (OD) pairs in the network. The two routing strategies that were previously mentioned were embodied in the formulations. The hub and spoke strategy will consolidate passenger demands on the least costly links in the network by abandoning the more costly ones. This consolidation process is accomplished by comparing the unit cost on the direct route between any given origin destination pair and all unit costs on potential routes that include a transfer. The least costly route weather it is a direct or a transfer route is then selected and passengers are assigned to it. This cost comparison in essence is really then nothing but a comparison between the two airline' routing strategies. The main advantage of this formulation is the fact that it keeps all options or route combination possibilities that can satisfy all travel 
demands in the system open for comparison. This advantage enables the formulation to do an exhaustive search for all routes that minimize the total cost of operations.

Due to the nature of air transportation, comparing unit costs turn out to be a great idea. This is so because once a flight departs, the airline incurs its cost regardless of how many passengers were flying on that flight. Therefore, the consolidation process reduces the number of flights required to satisfy all demands throughout the network. Reducing the number of required flights will then eventually reduces the total operating cost of the airline. Also, the usage of unit costs links the demand distribution throughout the network to the process of creating the network. This will result in the creation of a network that is "demand driven". Hence, if the demand distribution change, the network shape will also change to accommodate the new demand distribution. This is so because any change in the demand distribution will cause a change in the unit costs.

The following two examples will help in demonstrating the consolidation effects and compare this thesis approach of constructing the transportation network with the traditional approach of the urban transportation planning process. The first example will also prove numerically that the results produced by the formulation are the least costly among all other possible alternatives. The second example in particular will show that the formulation is robust and it can produce good results for networks with different number of airports and costs of operations. 


\section{Example 1:}

Let us assume that we want to find the optimal routing strategy for trips on the simple network shown in figure 3.6. Assuming that we have unit demands between all (OD) pairs in both directions in the network and the costs of the vehicle or aircraft trips are as indicated in the figure. To prove the validity of the formulation, all possible solutions to the problem will be generated at first and then they will be compared with the solution produced by the formulation.

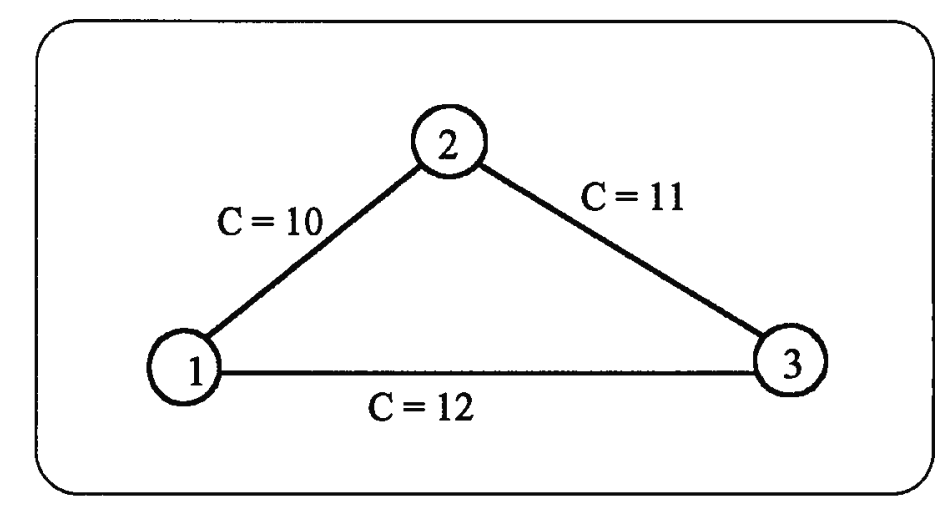

Figure 3.6 : Example 1, Network Problem

\section{Solutions :}

The traditional approach of the urban transportation planning process was used to produce the first solution. This problem was solved by constructing the minimum cost trees for each node at first and then these trees were overlaid together to get to the final shape of the network as shown in figure 3.7. 


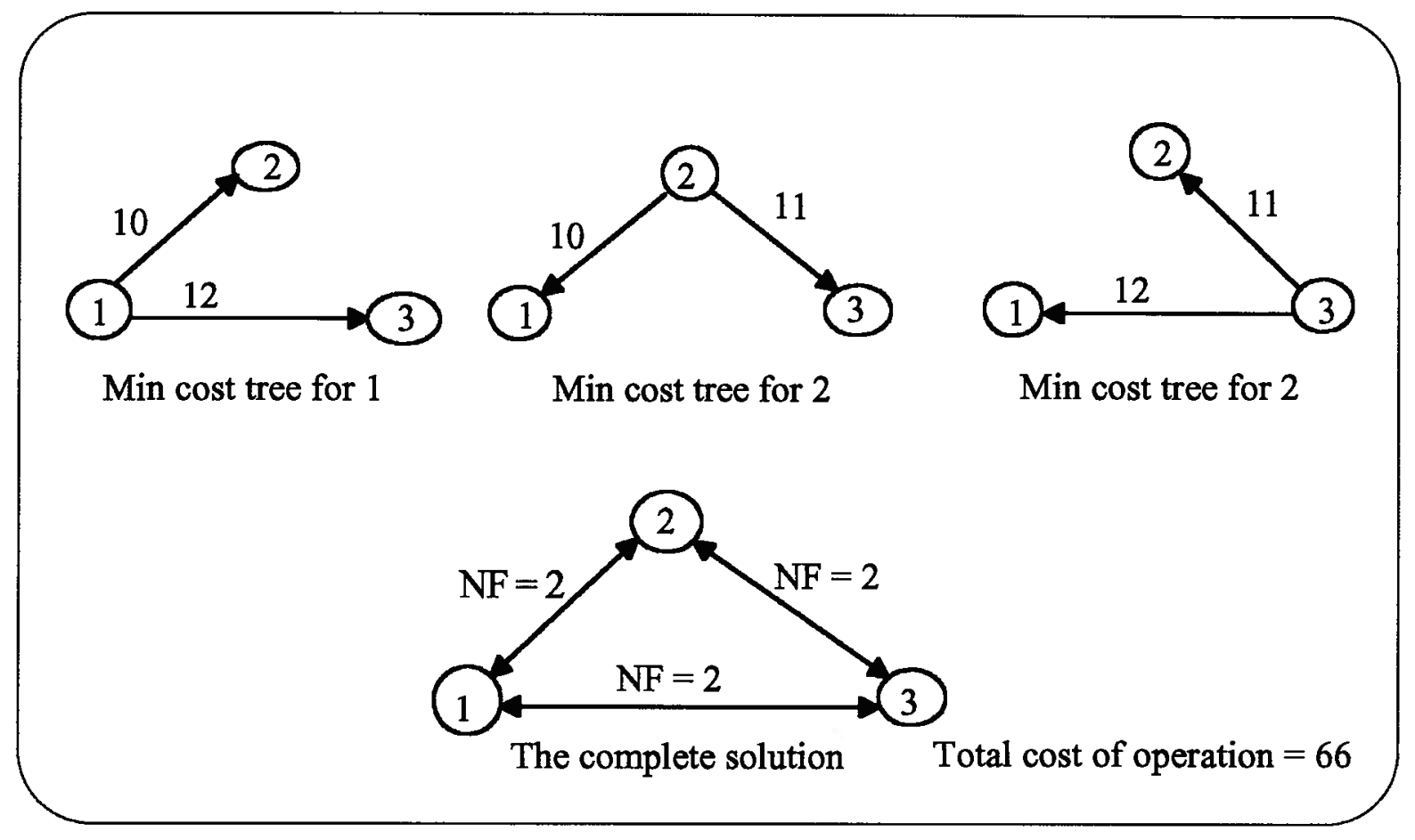

Figure 3.7 : The Tradditional Urban Planning Solution

According to the first solution, three Bi-directional links must be established between the three nodes. This will require a total of six flights to serve the demand. The total cost of operating the system will then be 66 units. This solution while it might be suitable for assigning personal trips using the automobile it is not suitable for assigning them using the aircraft. This is so because of the nature of air travel where personal trips can be consolidated and the number of required flights can then be reduced as discussed before.

A second possible solution is to use the hedgehopping strategy by creating a "loop of flights" to serve the network. This is shown in figure 3.8. This solution will require a total of 4 flights. The first flight will depart from 1 to 2 and it will take 2 passengers. One passenger would be travelling between 1 and 2 and the second passenger will be travelling between 1 and 3 through 2 . The second flight will depart from 2 to 3 and it will take 3 passengers. The first 
passenger is the one travelling to 3 from 1 , the second is the one travelling between 2 and 3 , and the last passenger is the one travelling between 2 and 1 through 3 . The third flight will depart form 3 to 1 and it will take 3 passengers. The first passenger is the one travelling to 1 from 2 , the second is the one travelling between 3 and 1, and the last passenger is the one travelling between 3 and 2 through 1 . The fourth flight will depart form 1 to 2 and it will take the passenger travelling from 3 to 2 through 1 . The total cost of operating this system will then be 43 units. Although, this solution consolidates the demands and reduces the number of the total flights required it is not the least costly solution that can be found. In fact, the least costly solution is going to be the one produced by the formulation.

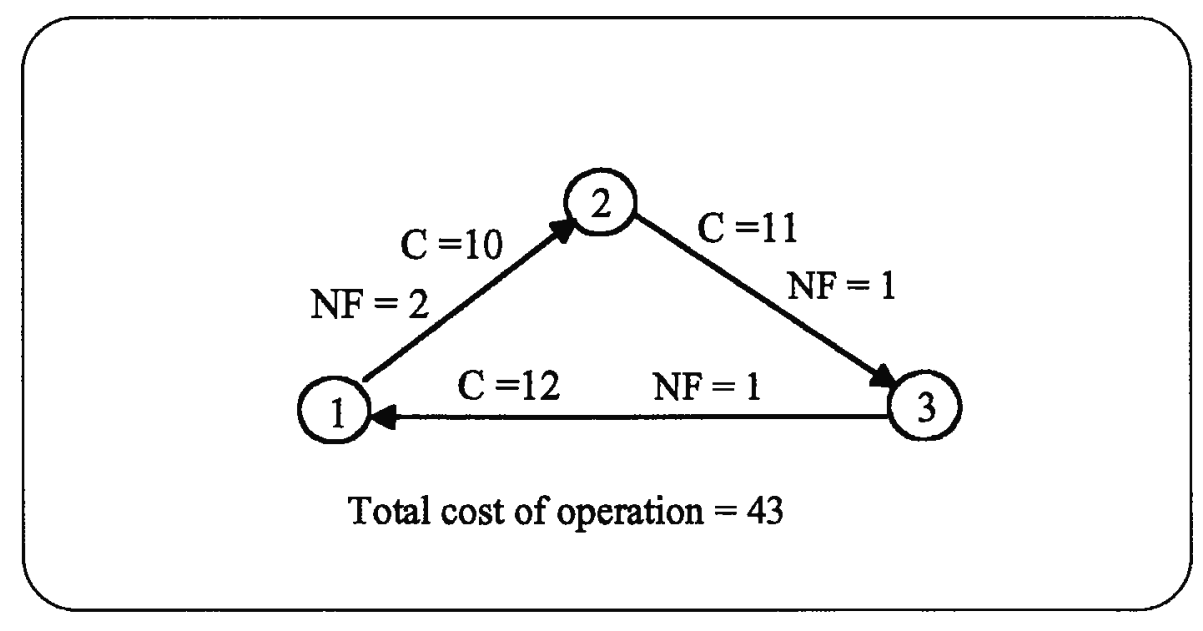

Figure 3.8: The Loop Solution

The third and fourth solutions (as presented in figure 3.9) can be established by using airports number 1 and 3 as transfer airports respectively. A total of four flights will be required for each solution with a total cost of operations of 44 and 46 units respectively. 


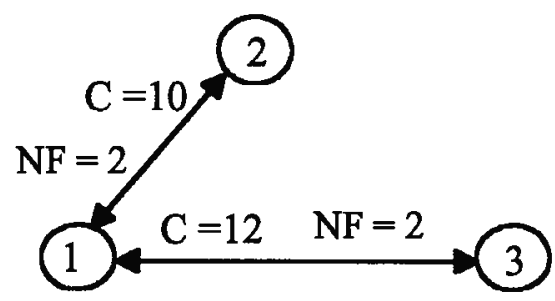

Total cost of operation $=44$

The third solution

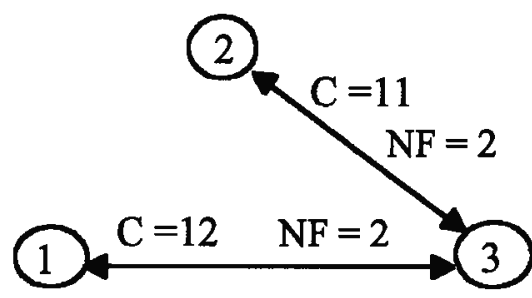

Total cost of operation $=46$

The fourth solution

Figure 3.9: The Third \& Fourth Possible Solutions

Finally, Figure 3.10 presents the last possible solution which is produced by the formulation. The solution is established by using airport number 2 as the transfer airport in a manner similar to the fourth and fifth solutions. This solution however is the optimal one since its resulting total cost of operation is only 42 units which is the least cost among all alternatives.

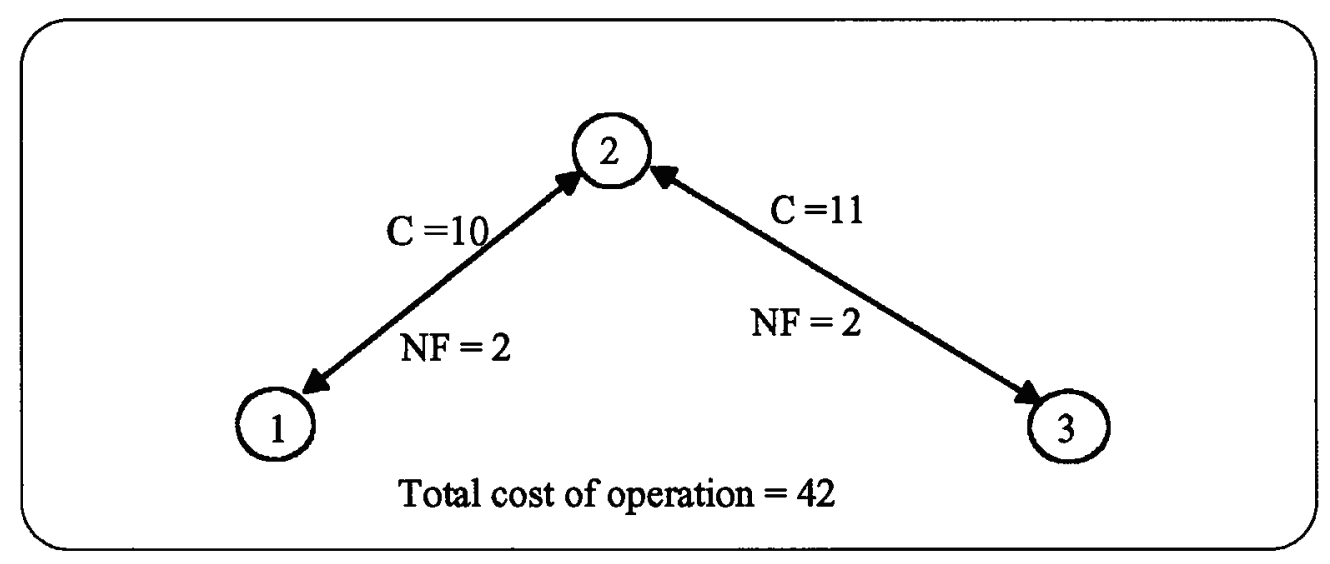

Figure 3.10: The optimal Solution For Example 1

\section{Example 2:}

This example is particularly designed to show the robustness of the formulation and its ability to react to and handle different networks. As can be seen in Figure 3.11, the same network of the first example is used with the exception that a fourth airport is added. Also the 
cost of operations on the new links connecting the fourth airport are less than the operation costs on other links.

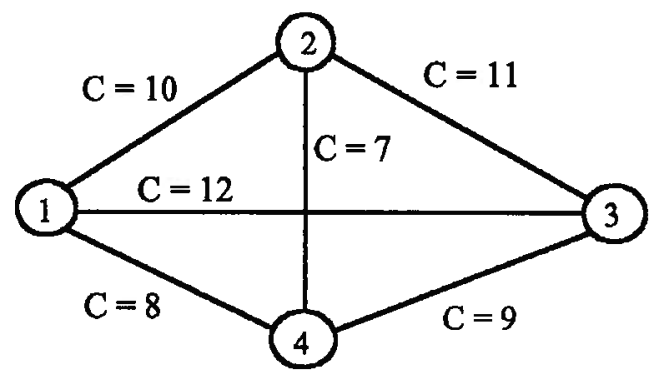

Figure 3.11 : Example 2, Network Problem

Solution

The solution is presented in Figure 3.12. It shows that the formulation has reacted to the introduction of the fourth airport with its less costly links. Flights were then shifted away from the previous links which were identified as least costly in example 1 and assigned to the new links.

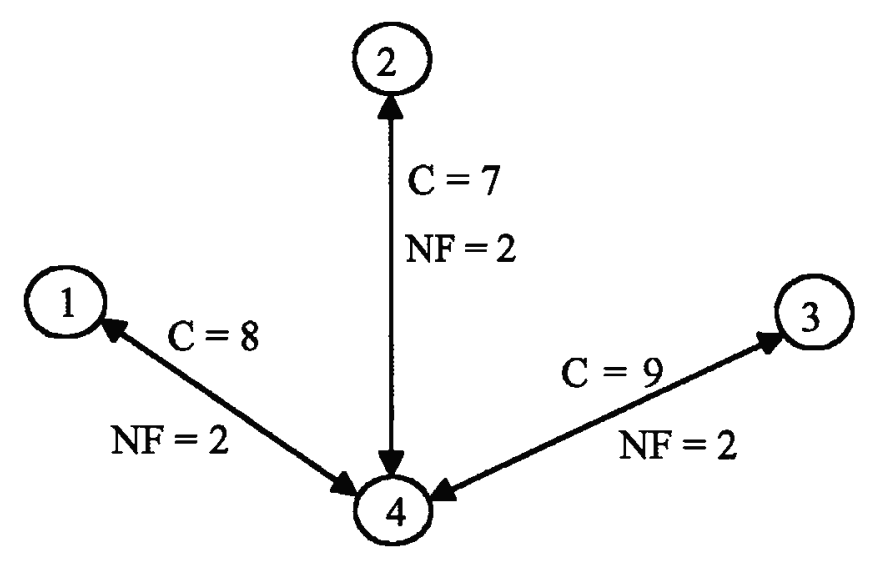

Total cost of operations $=48$

Figure 3.12: The Optimal Solution For Example 2 


\subsubsection{Discussion Of The Methodology}

To summarize, the construction of "an efficient air transport network" will be accomplished by conducting an exhaustive search for the least costly routes among all possible direct and transfer route alternatives. This search is done by comparing the total unit cost of transporting passengers according to the two routing strategies and by selecting the least costly routing strategy that can serve the demands between each orign-destination pair in the network. The usage of unit costs links the demand distribution throughout the network to the process of creating the network. This will result in the creation of a network that is "demand driven". Hence, if the demand distribution change, the network shape will also change to accommodate the new demand distribution. This is so because any change in the demand distribution will cause a change in the unit costs. Depending on the type of network design required, different cost elements can be included in the total unit cost. For example, if the network is intended to minimize the airline operating cost then only the airline operating costs are considered. Possible routes are those that offer a direct connection or a connection through a transfer airport. The number of transfers for any trip is limited to one merely for the convenience of passengers. However, this limitation will definitely eliminate any hedgehopping in the network. Such elimination is reasonable when the network size is relatively small as is the case in the case study of the thesis, or when the convenience of the passengers in terms of reducing their transfer time is among main objectives of creating the network. In larger networks however, hedgehopping may need to be considered for any given reason. In that case, the restriction on the number of transfer must be relaxed by modifying the model. All airports in the network that have travel interaction with both the origin-destination pair at hand can be considered as 
transfer airports. Assuming that all trips between any two demand locations (origin and destination) can only be assigned to one path which has the least unit cost. In effect a process similar to the "all or nothing trip assignment" of the urban transportation planning process will be used to create the network.

One potential advantage of this formulation that is worth to note is the noticeably short computation time. This may be a result of the limited number of sets of constraints imposed on the objective function.

\subsubsection{Computerizing The Formulation}

Writing all the elements of the above objective functions and constraints for a large network with many airports can be a cumbersome task. It is possible to have a huge number of alternative route combinations that trips can be assigned to between the different demand locations, hence mistakes can occur. For this reason a computer program written in " $\mathrm{C}$ " language was developed to write the complete forms of the objective function and constraints as presented in appendix (B). A detailed discussion of the program will be provided in the following chapter. 


\section{THE COMPUTER PROGRAM}

This chapter outlines the mentioned computer program developed to formulate the routing algorithms and to analyse the design networks. The program's data inputs and output files along with its flow chart and the tasks that it is suppose to accomplish will be presented. In addition, network performance measures are developed. These measures will help analysing the different network implications on airline operations and passenger service.

\subsubsection{The Program}

As previously mentioned, writing all the elements of the routing algorithms for a large network with many airports can be a cumbersome task. Also, when dealing with networks that have large numbers of airports to be served, it is possible to have a huge number of alternative route combinations that trips can be assigned to between the different demand locations. As the number of possible combinations increases mistakes in the formulation can occur. For this reason a personal computer based program written in " $\mathrm{C}$ " language was developed to write the complete forms of the objective function and constraints as presented in appendix (B). The program is also designed to help its user in evaluating the performance of the designed networks against the existing network. Figure 4.1 shows the program flowchart which will help in showing the interrelationships between the various tasks involved and it will also help in facilitating a better understanding of the program. 


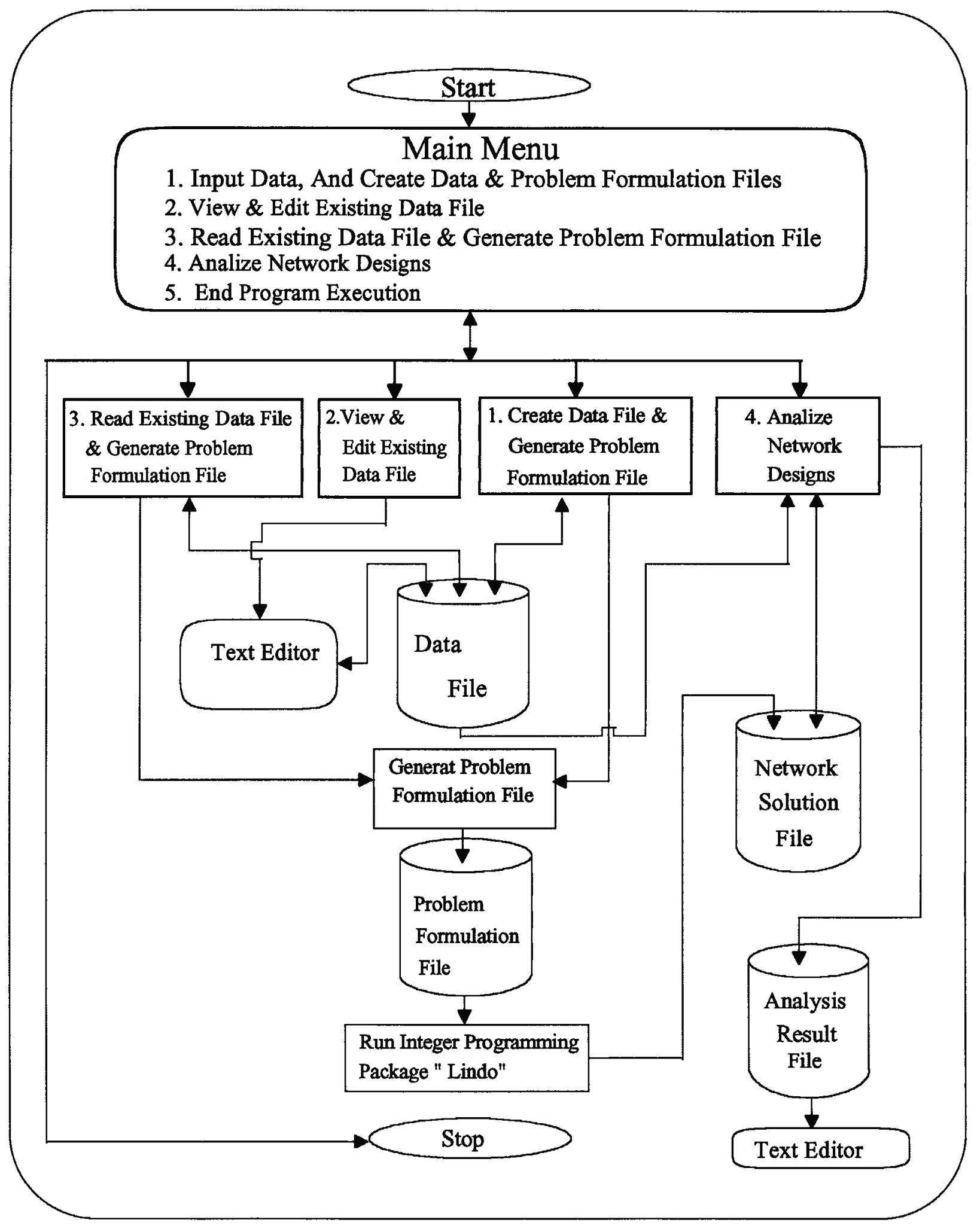

Figure 4.1 : Program Flowchart 
Before running the program it is necessary to assign numbers to all airports in the network. Those numbers will be used by the program to identify the different airports and to also identify the links between them. It is also recommended to prepare the necessary information about traffic demands, flights operating cost, travel times and distances between the different origins and destinations in matrix formats. This is important to ease and speed the data entry process as required by the program. The program was designed to be user friendly and to accomplish the following four tasks:

1. Input data, and create data and problem formulation files.

2. View and edit existing data files.

3. Read existing data files and create problem formulation files.

4. Analyse the resulting network designs

As seen in the flowchart, the program is designed to start by presenting its main menu which will enable the user to select one of the four mentioned tasks or to stop the program. The following will be a detailed discussion of each task.

\subsubsection{Input Data, And Create Data And Problem Formulation Files}

If no "Data File" is already in existence, the user must select the first task to create one. In that case, the program will request the user to enter a data file name and a problem formulation file name which the program will create for later use. The names will then be assigned to those files with the extension "DAT" added to the data file name and the extension 
"FUN" added to the problem formulation file name. The program will then ask about the required network design, i.e whether the network design is intended to suit the passenger, the airline, or both. And it will also ask for the total number of airports in the network and the value of time or the hourly wage rate. After this, a new screen as presented in Figure 4.2 will appear. On this screen, the airport indices (numbers) are generated automatically and the following information will then be requested for entry as the cursor moves and blinks under the needed information :

1. Passengers flow (Pax) between the given origin destination pair. This is necessary to determine the demand distribution throughout the network and to calculate the unit costs of transport. In our case study, weekly demands are used because in some parts of the network the daily demands are very low. Weekly demands were obtained by dividing annual demands as given by the airlines by 52 .

2. The cost of operating a direct single flight $(F C)$ according to the aircraft type used between the given origin destination pair. This is necessary to calculate the total and unit cost of transport. In our case study, actual costs of flying in thousands of Saudi Riyals were provided by the airline.

3. The number of seats $(S)$ according to the aircraft type used between the given origin destination pair. This is necessary for calculating the number of required flights $(N F)$ which is equal to the number of passengers divided by the number of seats and always rounding up the results in our case study to accommodate all passengers.

4. The average block time in hours $(B T)$ of the flight between the given origin destination pair. This is necessary to minimize the travel times of passengers and to convert time units to cost units. In our case study this data element is provided by the airline. 
5. The distance in miles $(D)$ between the given origin destination pair. This is necessary to calculate the costs of operations per passenger mile or seat mile. In our case study this data element is provided by the airline.

\begin{tabular}{|c|c|c|c|c|c|c|c|c|c|c|}
\hline \multicolumn{11}{|c|}{$\begin{array}{l}\text { Number_of_airports: } 25 \\
\text { Value_of_time: } 28.40\end{array}$} \\
\hline From & To & $\begin{array}{l}\text { Flow } \\
\text { Pax }\end{array}$ & $\begin{array}{l}\text { F.Cost } \\
* 1000\end{array}$ & Seats & $\begin{array}{l}\text { Time } \\
\text { hr }\end{array}$ & $\begin{array}{l}\text { Dist } \\
\text { Mi }\end{array}$ & From & To & $\begin{array}{l}\text { Flow } \\
\text { Pax }\end{array}$ & $\begin{array}{l}\text { F. Cost } \\
{ }^{*} 1000\end{array}$ \\
\hline 1 & 2 & 0 & 0.00 & 0 & 0.00 & 0 & 2 & 1 & 0 & 0.00 \\
\hline 1 & 3 & 0 & 0.00 & 0 & 0.00 & 0 & 3 & 1 & 0 & 0.00 \\
\hline 1 & 4 & 0 & 0.00 & 0 & 0.00 & 0 & 4 & 1 & 0 & 0.00 \\
\hline 1 & 5 & 2 & 8.33 & 102 & 0.49 & 125 & 5 & 1 & 2 & 8.33 \\
\hline 1 & 6 & 77 & 66.97 & 102 & 1.81 & 607 & 6 & 1 & 77 & 66.97 \\
\hline 1 & 7 & 0 & 0.00 & 0 & 0.00 & 0 & 7 & 1 & 0 & 0.00 \\
\hline 1 & 8 & 0 & 0.00 & 0 & 0.00 & 0 & 8 & 1 & 0 & 0.00 \\
\hline 1 & 9 & 0 & 0.00 & 0 & 0.00 & 0 & 9 & 1 & 0 & 0.00 \\
\hline 1 & 10 & 0 & 0.00 & 0 & 0.00 & 0 & 10 & 1 & 0 & 0.00 \\
\hline 1 & 11 & 0 & 0.00 & 0 & 0.00 & 0 & 11 & 1 & 0 & 0.00 \\
\hline 1 & 12 & 0 & 0.00 & 0 & 0.00 & 0 & 12 & 1 & 0 & 0.00 \\
\hline 1 & 13 & 0 & 0.00 & 0 & 0.00 & 0 & 13 & 1 & 0 & 0.00 \\
\hline 1 & 14 & 404 & 37.13 & 258 & 0.79 & 106 & 14 & 1 & 442 & 37.24 \\
\hline 1 & 15 & 0 & 0.00 & 0 & 0.00 & 0 & 15 & 1 & 0 & 0.00 \\
\hline
\end{tabular}

Figure 4.2 : Input Screen, Typical Example.

Upon the completion of the data entry process, the program will then provides its user a chance to enter any specific side constraints, i.e, include or exclude any specific link or links between any given pair of origin and destination airports in the final design of the network. Those side constraints are important because they will allow the user to examine the effects of creating different situations such as developing a specific airport as a hub on the efficiency of 
the network. The program will then create a "Data File" which can be viewed and edited later on if necessary (see appendix (A)). After creating the data file, the program will call a subroutine to generate the "Problem Formulation File" which can be seen in the appendix (B). The subroutine embodies in it the general objective function and all constraints equations as well as all the different cost functions that were mentioned in the methodology chapter. As shown in the flowchart, the problem formulation file which contains the formulated objective function and constraints has then to be used as an input for a linear optimization package called "Lindo" which is available on the Mainframe computer of the university. Lindo will solve the problem and will then provide the network design in the "Network Solution File" which contains the set of links between the different airports that comprises the final network design. The solution file is presented in appendix (C). As shown in the flowchart, once a selected task is accomplished, the program reverts back to the main menu. The user will then have the chance to either select another task, or to stop the program.

\subsubsection{View And Edit Existing Data File}

When entering a large set of data as required by this program, mistakes can happen. For this reason, the program is designed to view and edit any existing data file. Once this option is selected, the program asks the user to enter the name of the data file to be edited. The user

response will then prompt the program to start a text editor namely "Microsoft Dos Edit" and open the data file for editing. The user must then edit and save the file to return to the main menu. 


\subsubsection{Read Existing Data File And Create Problem Formulation File}

Once this option is selected, the program will ask its user to enter the names of the data file and the problem formulation file. All information in the data file will then be used by the previously mentioned subroutine to create the problem formulation file.

\subsubsection{Analyse Network Designs}

In order to provide a better understanding of the network designs and to evaluate them against an existing network, the program is designed to read the network solution files and provide the analysis results in the "Analysis Result File" ( see appendix (D)). To start the analysis, the program will first read the data file in order to retrieve all information relating to the traffic demand levels and flight costs. Network analyse will be conducted according to two analysis categories, these are:

1. Link analysis. This analysis category is used to evaluate the impact of implementing the new network designs on traffic flows over the different links that make up the network. Airline operating performance and passenger' services will be evaluated on each link. In this analysis category, all links that make up the network are scanned form the network solution file. The numbers of passengers using each link either as a direct link or as a transfer link between their origin and destination airports are read from the data file and added together to give the total number of passengers assigned to each link. The required number of flights on each link can then be determined by 
dividing the total number of passengers assigned to the link by the number of seats according to the aircraft type that is going to be used by the airline on that link. It is assumed that the airlines will continue to use the same type of aircraft that it is currently using between the origin and destination airports. The user however can evaluate the impact of using different aircraft on the shape of the network. Since flights are indivisible, all fractions in the division result are rounded up to accommodate all passengers. In addition, some performance indicators as will be seen later were created to evaluate the impact of implementing any new network design on flight operations and passengers' services on the different links.

2. Entire network as a system. This analysis category is intended to evaluate the performance of the network as a whole system. The impacts of implementing any new network design on the airline operations and passenger' service is analysed. In this analysis category, information relating to link operations are either added or averaged to quantify network performance. Various performance indicators were utilized to evaluate the different impacts.

\subsubsection{Performance Indicators And Analysis Data}

The airline industry has established a set of performance indicators to evaluate different aspects of airlines operations and performance. In this thesis however, the main test of improvements in airline operations as a result of implementing the new networks is the reduction in the total and unit costs of providing the service. Improvements in passenger 
services are mainly measured in terms of flight frequencies, and schedule delays. These improvements will reduce the passenger's costs of travel. The following will be a presentation of the data items to be analysed and their related performance indicators which were utilized in this thesis to evaluate the different impacts of implementing all the new design networks:

1. Number of passengers (Pax). This is the total number of passengers using each individual link including direct and transfer passengers. The number is necessary to determine the minimum number of flights that can accommodate the demand. It is also important for calculating unit costs as will be seen later.

2. Number of flights $(N F)$. The total minimum number of required flights on each link is important for determining schedule delay time of passengers. In this thesis, the number is calculated as: $N F=P A X / S$ where $S$ being the number of seats on the aircraft. Given the indivisibility of flights, the result must be rounded up to accommodate all passengers. The number is also used for calculating the total operating cost for each link and the entire system. $N F$ is calculated for each link as shown above, and for the entire system by adding up the costs of flights on individual links.

3. Average schedule delay time $(A D T)$. As previously defined, this is used as a measure of the level of service being offered to passengers. In this thesis, passenger's travel demand was assumed to be uniformly distributed over the week ${ }^{44}$, hence, $A D T$ is calculated as: $A D T=$ total operating hours in the week $/ 2 N F . A D T$ is calculated for each link as shown, and for the entire network system by adding the link numbers to get the total system delay time. Average system schedule delay time for all links is also calculated by dividing the total system delay by the number of links in the network.

4. Total operating cost $(T C)$. This is the major operation's improvement measures. For link analysis it is calculated as: $T C=F C^{*} N F$. The total system cost is then obtained by adding the costs of providing the service on all links. 
5. Passenger miles flown ( $\operatorname{Pax} M i)$. Because not all flights have the same flight distance, passengers on different flights are assigned different "weights". This number is defined as the sum of the products obtained by multiplying the number of passengers carried on each flight by the flight distance ${ }^{45}$. The number can also be used as a measure of the dispersion of the network.

6. Available seat miles (ASMi). This is a measure of the capacity provided by the airline. It is defined as the sum of the products obtained by multiplying the number of passenger seats available for sale on each flight by the flight distance ${ }^{46}$. This measure is calculated for the system analysis category, but it is also used to calculate the cost per seat mile for each link.

7. Passenger load factor $(L F)$. This is a measure of the capacity utilization. It is defined as the percentage of passenger miles from the available seat miles. Hub and spoke networks which usually consolidate passengers demands usually has higher load factors than point to point connection networks.

8. Cost per seat mile $(C / S M i)$. This is a measure of the airline productivity. It is calculated as: $\quad C / S M i=T C / A S M i$. This measure is calculated for both the link analysis and the system analysis categories. Network configurations that provide smaller numbers usually indicates better performance. However this can be misleading because as the airline provide more capacity in some segments of the market, this capacity might not be fully utilized.

9. Cost per passenger mile $(C / P a x M i)$. This is a measure of the airline productivity. It is calculated as: $\quad C / P a x M i=T C / P a x M i$. This measure is calculated for both the link analysis and the system analysis categories. A network configuration that provide smaller numbers usually indicates better performance. 


\subsubsection{Sample Output}

For illustration purposes, a sample of the outputs of the integer programming package "Lindo" and the "C" program will be presented below. As can be seen below, Lindo's output is a list of the routes that make-up the designed network. The $\mathrm{C}$ program output is comprised of two parts. The first part is a list of all the links in the network with the performance indicators associated with each link. The second part is the result of the calculations of all system performance indicators. The sample given below is an extract of the analysis result of the existing airline network in Saudi Arabia.

\section{Lindo's Output: "Network Solution File"}

\begin{tabular}{|c|c|c|}
\hline Y1T5 & 1.0000 & This indicates the presence of a direct route between 1 and 5 . \\
\hline Y1T18T6 & 1.0000 & This indicates the presence of a transfer route between 1 and 6 through 18 . \\
\hline \multicolumn{3}{|l|}{ Y1T14 } \\
\hline Y1T18 & & \\
\hline Y2T18T6 & & \\
\hline
\end{tabular}

The sample given above is a list of the routes that make up the network. The complete network files for the existing network and the proposed new networks are presented in appendix (C). 
2. The "C" Program Output : "Analysis File"

\begin{tabular}{|c|c|c|c|c|c|c|c|}
\hline $\begin{array}{l}\text { Link } \\
\text { Fr To }\end{array}$ & $\begin{array}{l}\text { Total } \\
\text { Flow }\end{array}$ & $\begin{array}{l}\text { Total } \\
\text { Flights }\end{array}$ & $\begin{array}{l}\text { Schedul } \\
\text { Delay }\end{array}$ & $\begin{array}{l}\text { ule Total } \\
\text { Cost (SR) }\end{array}$ & $\begin{array}{l}\text { Cost per } \\
\text { Seat Mile }\end{array}$ & $\begin{array}{l}\text { Cost per } \\
\text { Pax Mile }\end{array}$ & $\begin{array}{l}\text { Load } \\
\text { Factor }\end{array}$ \\
\hline 15 & 2 & 1 & 49.00 & 8330.00 & 0.65 & 33.32 & 0.02 \\
\hline 114 & 404 & 2 & 24.50 & 74260.00 & 1.36 & 1.73 & 0.78 \\
\hline 118 & 808 & 5 & 9.80 & 216150.00 & 0.59 & 0.74 & 0.80 \\
\hline 214 & 3134 & 16 & 3.06 & 730720.00 & 0.79 & 0.82 & 0.97 \\
\hline 218 & 3596 & 18 & $2.72 \quad 10$ & 1057320.00 & 0.63 & 0.64 & 0.99 \\
\hline 219 & 77 & 1 & 49.00 & 15680.00 & 0.52 & 0.68 & 0.75 \\
\hline
\end{tabular}

\footnotetext{
System Flights $=778$ System Cost $=32989960.00$ System Delay $=3736.87$

System Seat-Mi Cost $=0.56 \quad$ System Pax-Mi Cost $=0.60$

Average Delay $=26.69 \quad$ System Load Factor $=0.94$

Average Seat-Mi Cost $=0.66 \quad$ Average Pax-Mi cost $=\mathbf{2 . 5 4}$

Average Load Factor $=0.66$

Available Seat-Mile $=58683524$ Passenger-Mile Flown $=54875896$
}

The complete analysis files for the existing network and the proposed new networks are presented in appendix (D). 


\section{THE CASE STUDY}

As a case study, the domestic air transportation network of the Kingdom of Saudi Arabia which consists of 25 airports that have sparse demand levels between the majority of these airports were examined. The routes and links between the different airports in the network were restructured. This particular network was selected as a case study because as it will be shown in the discussion of the existing network conditions, this network represents a unique opportunity since the network size is large, demand is sparse, and there is no other fast alternatives of mass travel modes.

\subsubsection{Existing Network Conditions}

In general, any transport network plan that matches supply with demand has to be governed by constraints imposed by the availability of resources and economic efficiency. In this regard, the domestic air transport network plan of the Kingdom of Saudi Arabia is no exception. However when planning this network, the national airline (Saudia) was able to consider aspects that extend beyond economic efficiency. The network plan placed strong emphasis on objectives such as the country's political integration, the establishment of direct links between all population centres, and the social well-being of the population ${ }^{47}$. As a result,

all the Kingdom's centres of population are directly connected to the air transport system by their own airports. The majority of these airports are directly connected to one another even though demand levels may not necessarily justify direct connections. 


\subsubsection{The Structural Shape Of The Existing Network}

Saudia currently provide air transport services that link 25 airports with direct flights in most cases ${ }^{48}$. Figure 5. 1 shows the structural shape of the existing network. The network currently has a total of 172 routes connecting 172 city pairs of origins and destinations. Out of the 172 routes, only 32 routes include a transfer. A casual inspection of this network might mislead the reader into thinking that this is a multi hub and spoke network that has 23 hubs. However, by definition a hub airport is a focal airport that facilitates flights transfer between various origins and destinations. In this sense, Riyadh ( airport number 18 ), Jeddah (airport number 14 ), and Tabuk ( airport number 22 ) airports are the only hubs in the network. In reality, the network provides direct point to point service to 140 city pairs and only 32 city pairs are served by transfer routes. 


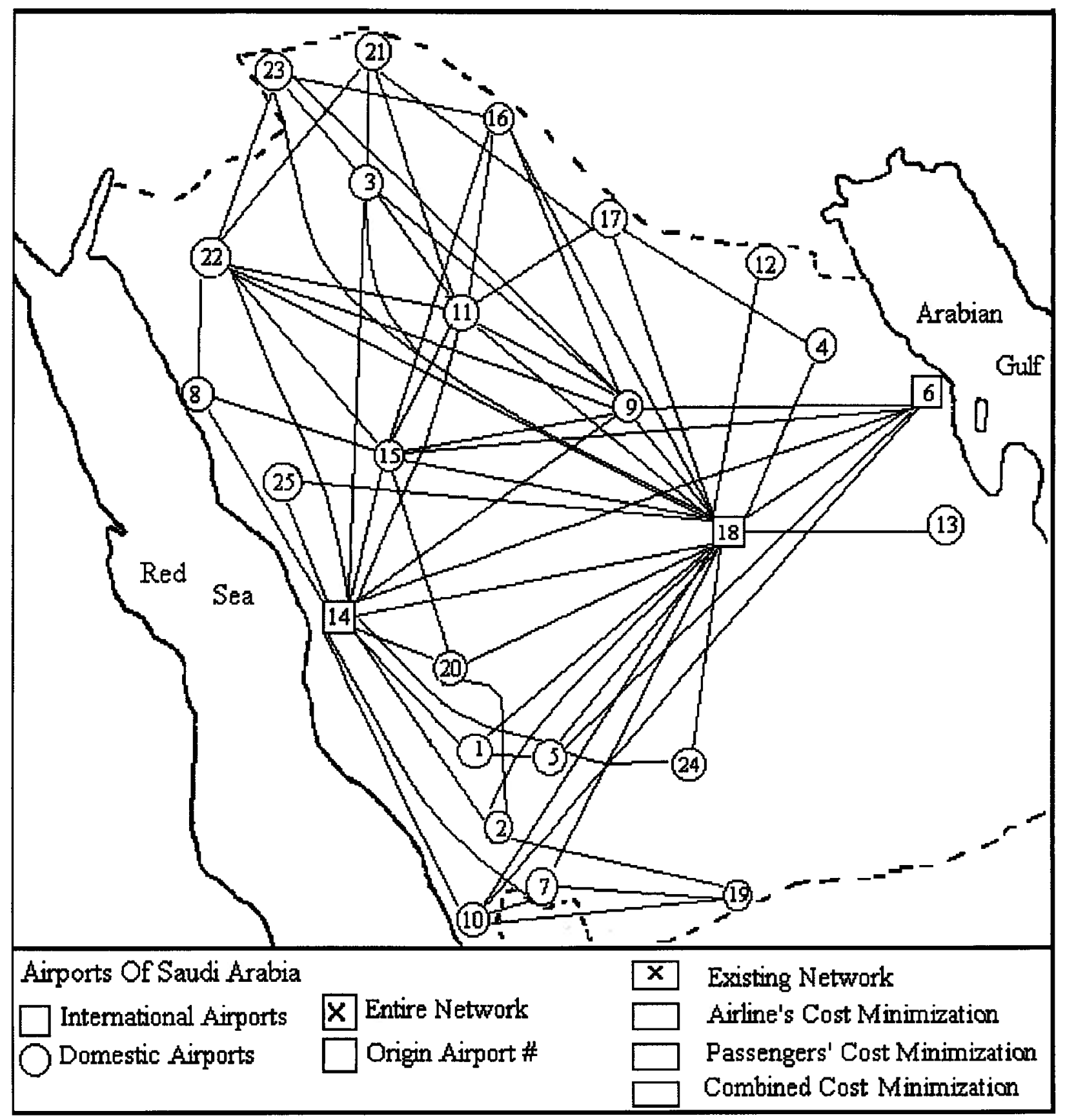

Figure 5. 1: The Existing Domestic Air Transport Network of The Kingdom of Saudi Arabia 


\subsubsection{Operational Characteristics Of The Existing Network}

Travel demand throughout the network with the exception of demands to or from Jeddah or Riyadh is sparse. A quick examination of the average weekly travel demand matrix provided in appendix (A) will show that travel demand between some city pairs is as low as 2 passengers per week. Saudia officials indicated that the airline currently provide scheduled air transport services between such city pairs as social services that are subsidized by the government. Given these low demand levels and the size of the Boeing 737 (102 seats) which is the smallest aircraft that the airline currently operates for scheduled service, load factors throughout the network are low. Figure 5.2 shows the estimated load factor distribution in the existing network if the airline operates only the minimum number of flights that can satisfy

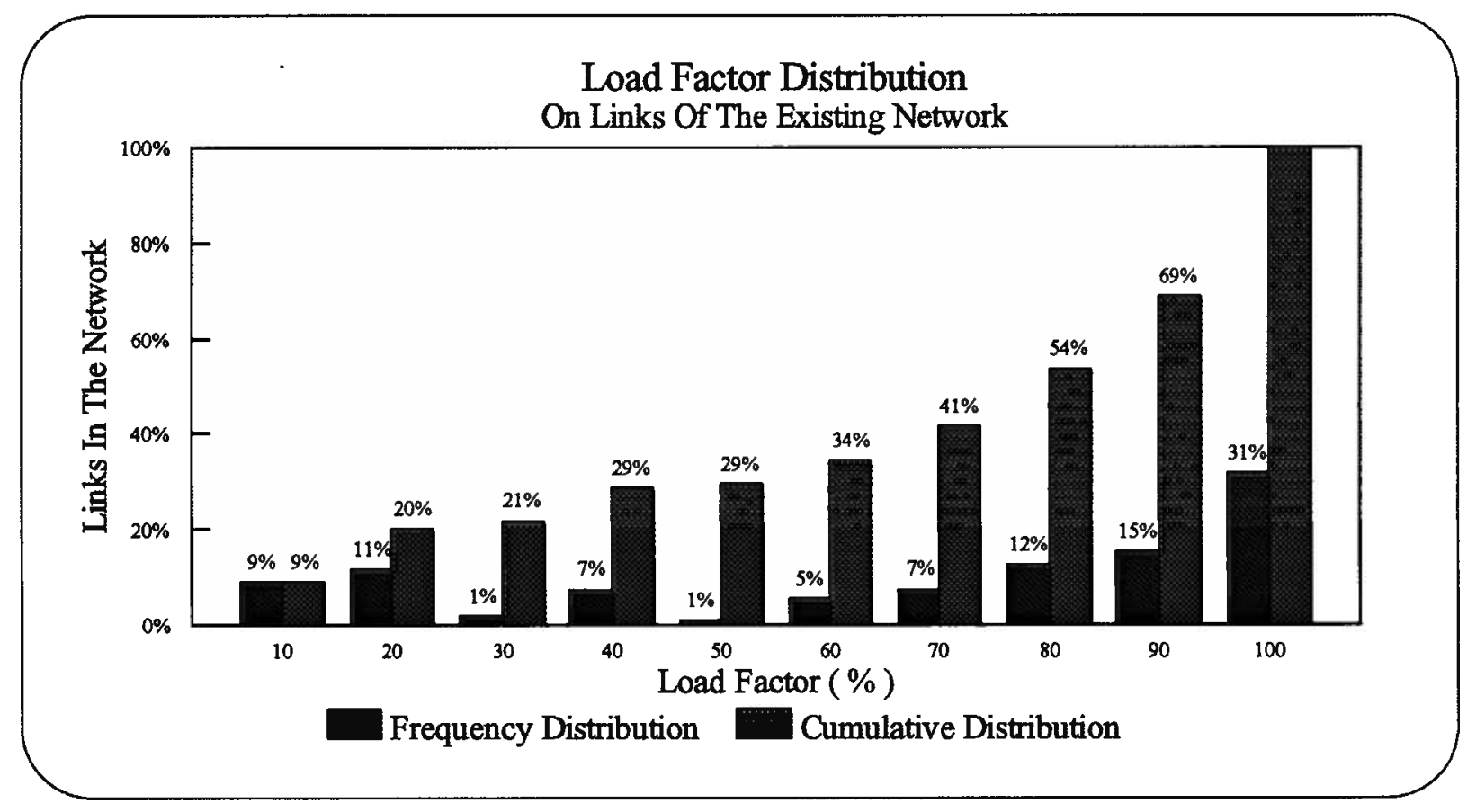

Figure 5. 2: Estimated Load Factors Distribution In Existing Network 
all travel demands. The figure indicates that up to $29 \%$ of links in the network have load factors between 1 and 40 percent. According to some Saudia officials, the actual operating load factors which were not available for this research are even less than those presented in the Figure. This is so because the airline currently operates more flights than the required minimum for different policy reasons. These policies include reasons that relates to the social well being of the population ${ }^{49}$. For this research the minimum number of flights is used as a base for conducting network analysis throughout the remainder of this thesis. This will help in providing a uniform basis for comparing the performance of the various network structures. Also, since there is no available data on how Saudia decides on the number of flights scheduled on each link, the minimum number is a viable solution to problems caused by the lack of data.

Figure 5. 3 shows the estimated number of flights distribution in the existing network if Saudia operates the minimum number of flights to improve its economic efficiency of operations.

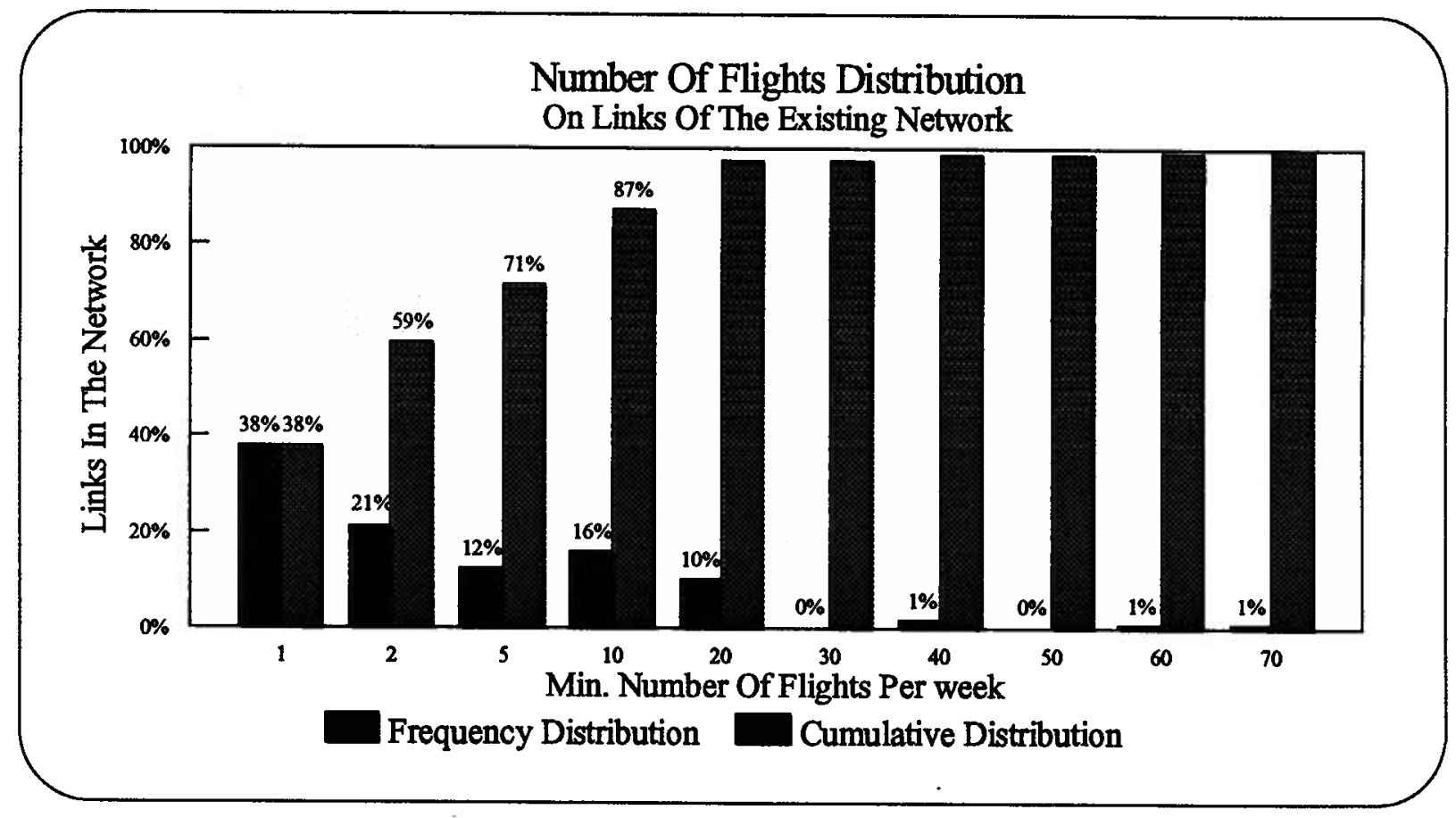

Figure 5. 3 : Estimated Number Of Flights Distribution In Existing Network 
In this case, Saudia will have to operate a minimum of 778 flights per week to meet all travel demands. The figure shows that $38 \%$ of the links will only support the operation of one flight per week for maximum economic efficiency. The figure also shows that up to $59 \%$ of all links in the network have only 1 or 2 flights per week. This will have an implication on the quality of service that will be offered to passengers. For example, the low number of flights on the majority of links will greatly increase the schedule delay in the entire system. In fact, if the airline only operates the minimum number of flights, the total schedule delay for all links in the network will be 3,736 hours per week. Figure 5.4 indicates that $38 \%$ of the links in the network would have schedule delay of 50 hours per week. The same figure also indicates that only $16 \%$ of the links in the existing network have schedule delay up to 5 hours.

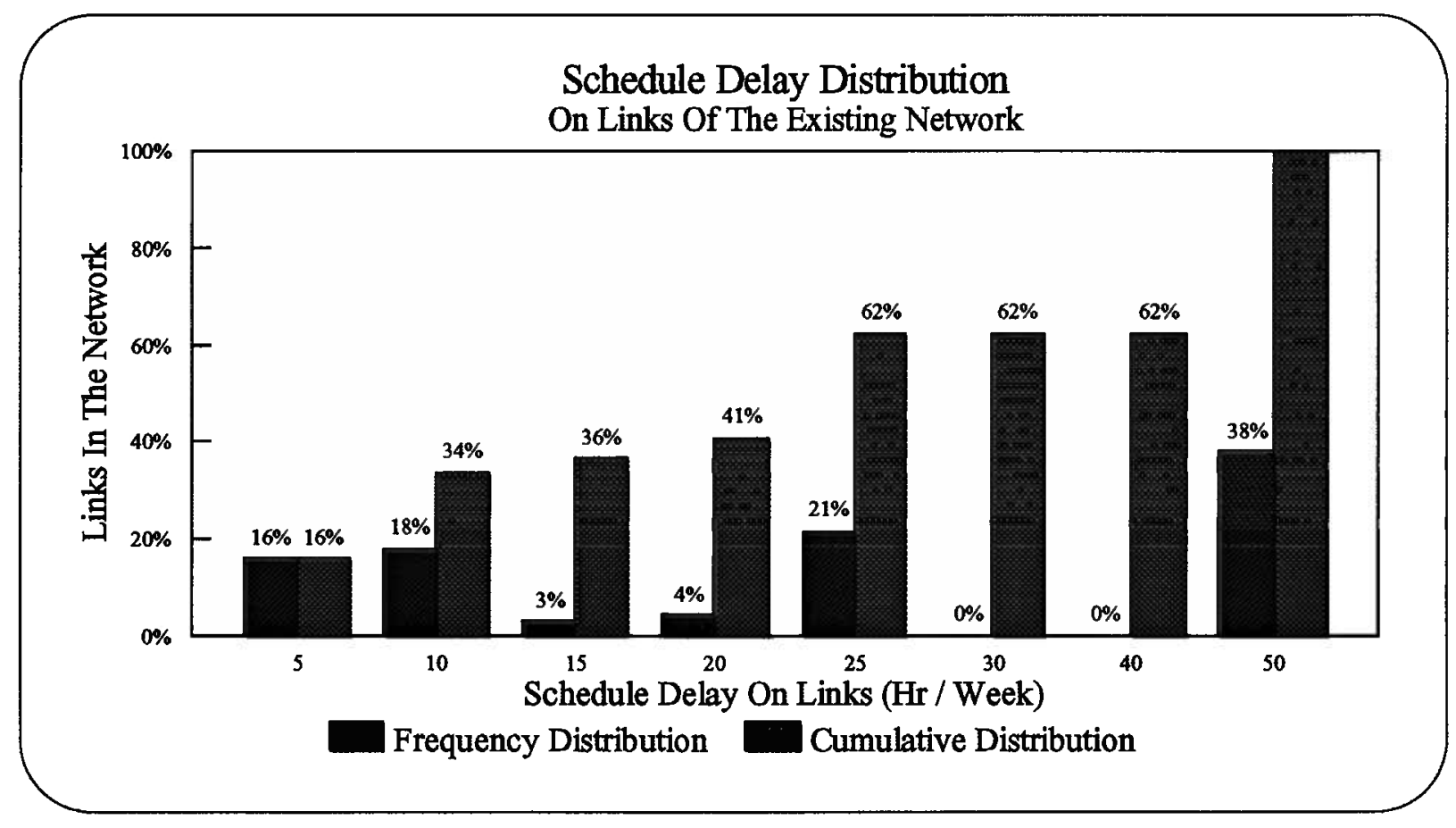

Figure 5. 4 : Estimated Schedule Delay Distribution In Existing Network

Finally, if Saudia operates the minimum required number of flights on each link, its total operating cost is estimated to be 33.0 millions Saudi Riyals (SR) per week. Figure 5.5 shows 
the estimated distribution of this cost in terms of the operating cost per passenger-mile. The figure shows a wide range of costs per passenger-miles that spreads between $0.5 \mathrm{SR}$ to $40 \mathrm{SR}$ on the different links. $57 \%$ of the links in the existing network have a cost that ranges between 0.5 SR to 1.0 SR. This can be explained by Figure 5.2 which indicated that approximately $58 \%$ of the links have load factors that ranges between $80 \%$ to $100 \%$.

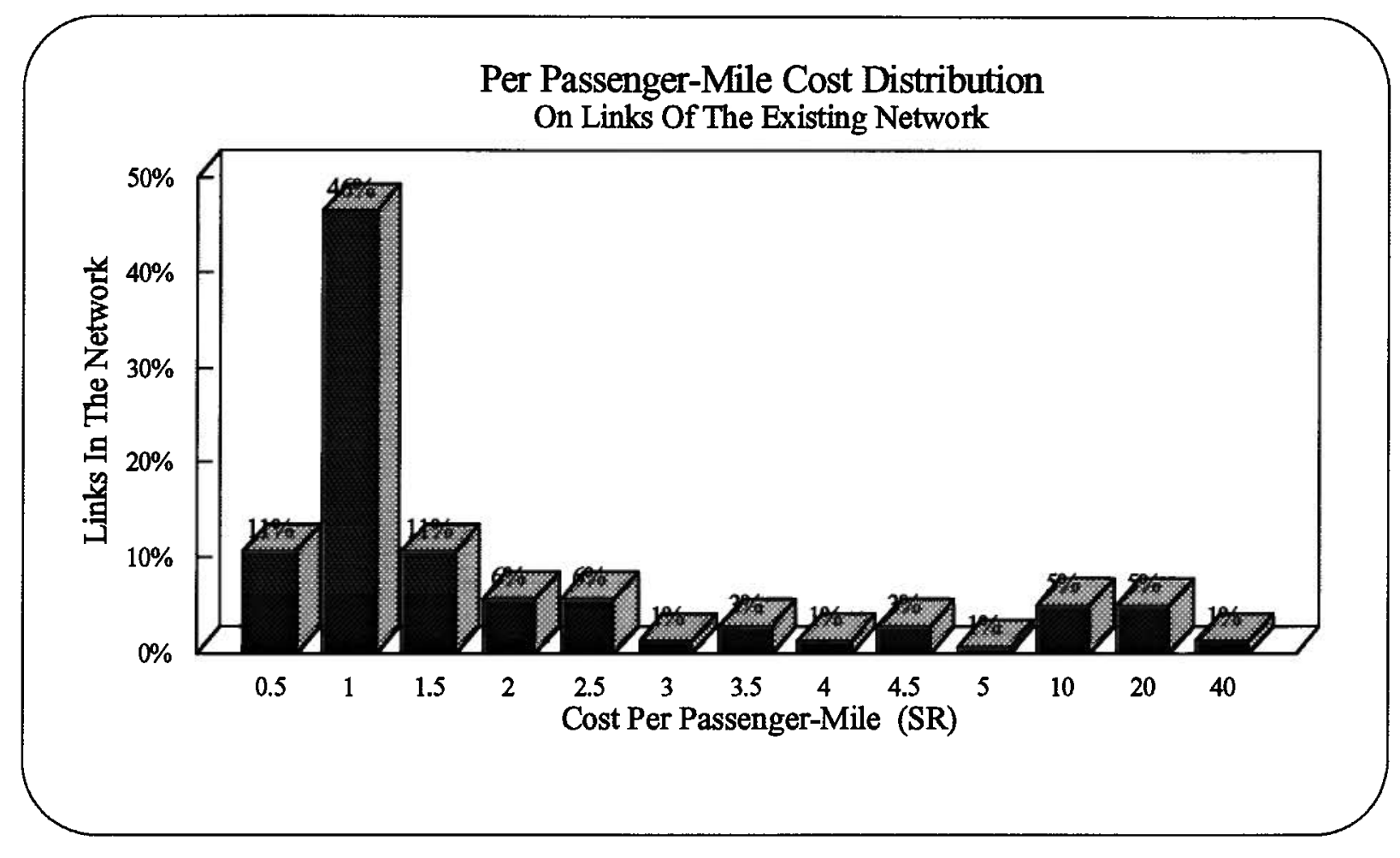

Figure 5.5 : Estimated Cost Per Passenger-Mile Distribution In Existing Network

To summarize, The existing network is mostly directly connected. About $81 \%$ of the routes in the network are direct connection routes. This high degree of direct connectivity coupled with the low demand levels and the size of the smallest aircraft that is currently being used by the airline clearly explain the low load factors that characterize the current operations. In return, the low load factors on the majority of flights explains the high cost of operations per passenger-miles. 


\subsubsection{Case Study Results}

Network restructuring was accomplished by applying the developed formulations. As a result, three new efficient network structures were constructed. Figure 5.6 shows the first network structure which was designed to minimize the total cost of the airline operations by consolidating travel demands through the usage of the hub and spoke routing strategy. Figure 5.7 shows the second network structure which was intended to reduce the cost of travel of passengers by reducing their travel time and schedule delay. When designing this network, it was assumed that ticket prices which passengers have to pay for the trips will remain unchanged regardless of the chosen routes. Figure 5.8 shows the third network structure which was developed to reduce the combined costs of both passengers and airline. This was done because as was seen in Figure 3.5, reducing the sum of the airline's and passengers' costs will produce the efficient level of service with its efficient economic cost.

\subsubsection{Analysis Of Results}

The following discussion will be a detailed analysis of the three efficient networks. The implications of each network structure on airline operations and passengers services will be presented. Items such as load factor distribution, number of flights distribution, schedule delay distribution, and operating cost distribution throughout the network will be discussed to compare the performance of each network structure. 


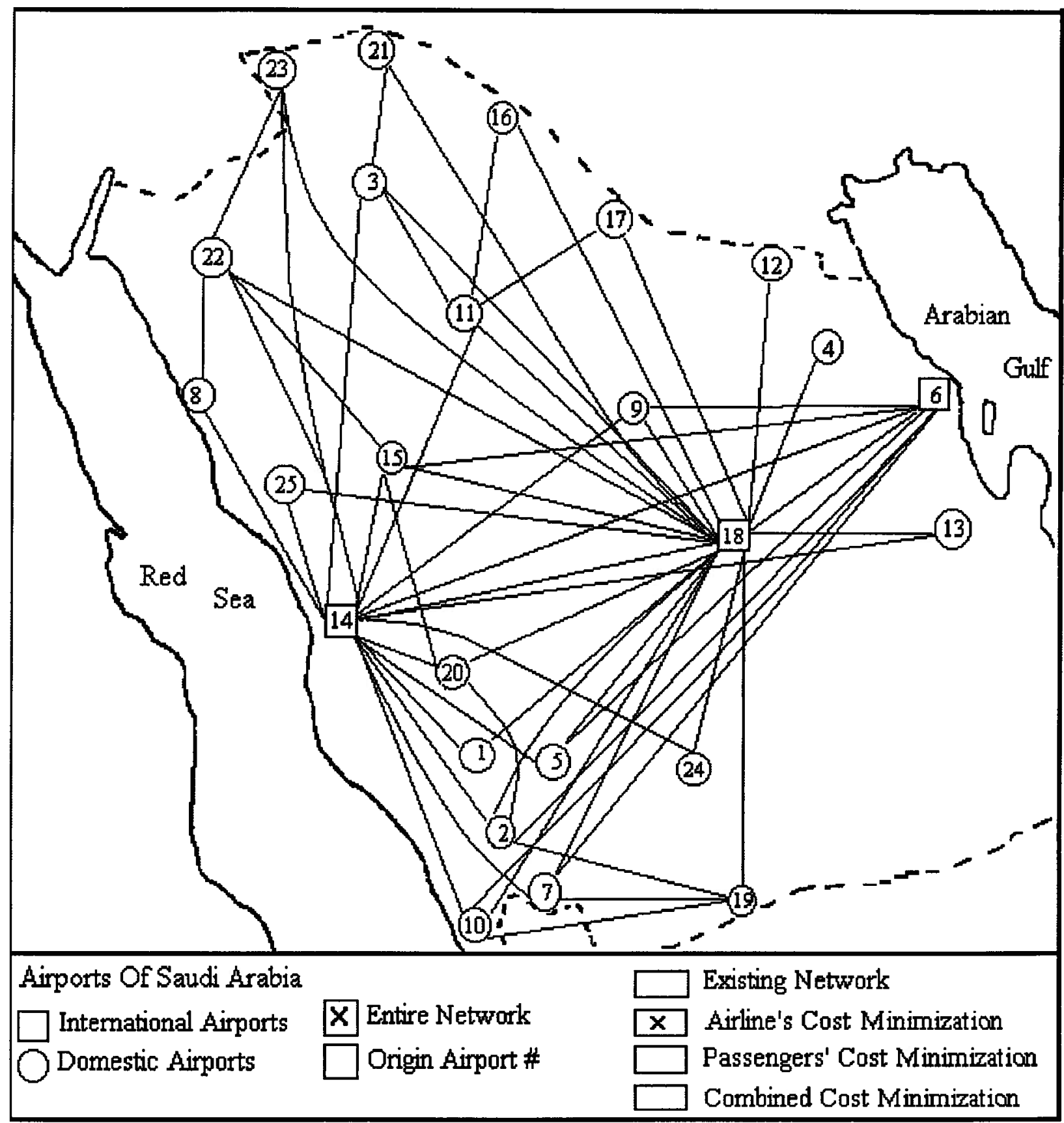

Figure 5. 6: Efficient Airline's Cost Network 


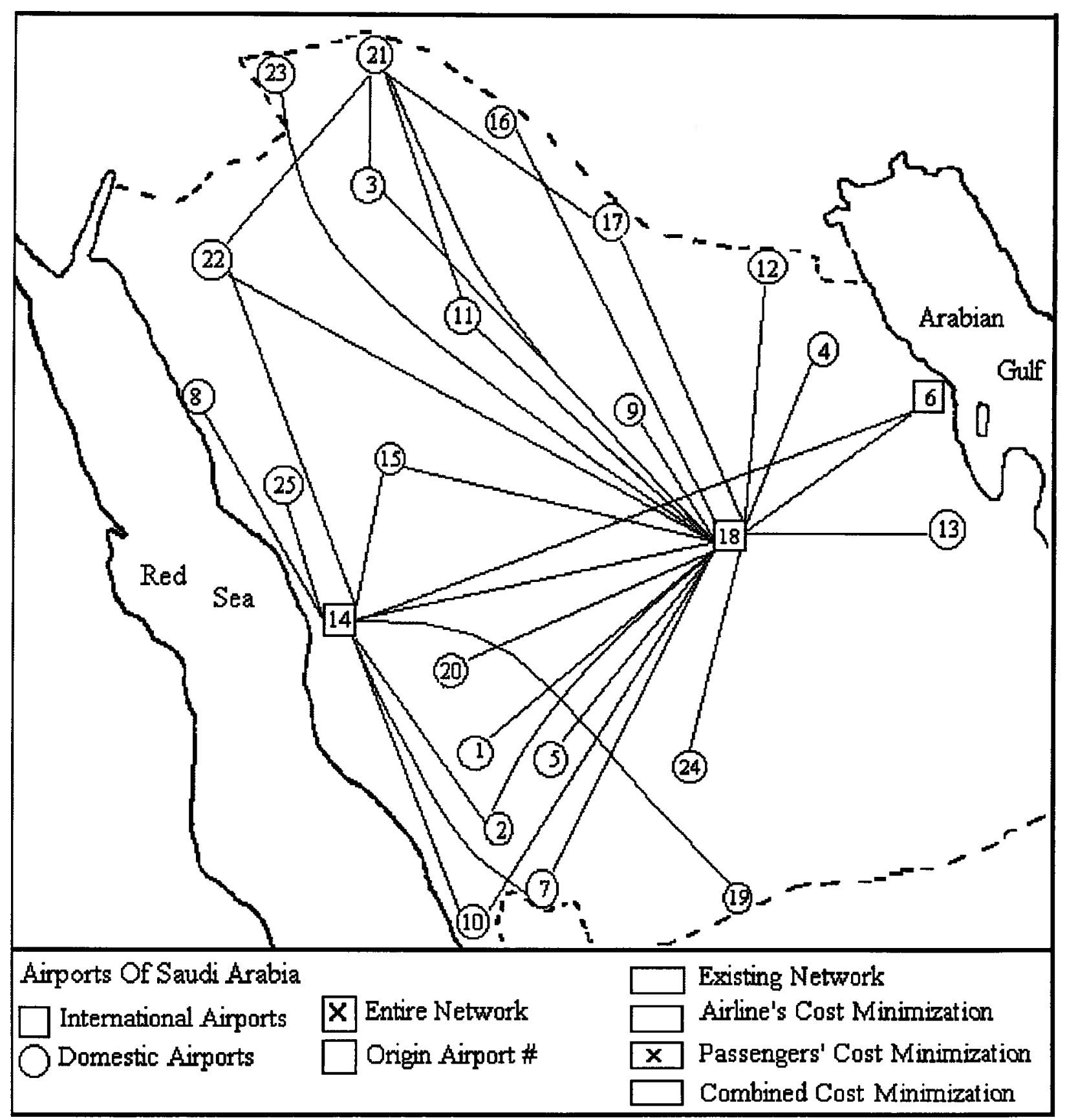

Figure 5. 7 : Efficient Passengers' Cost Network 


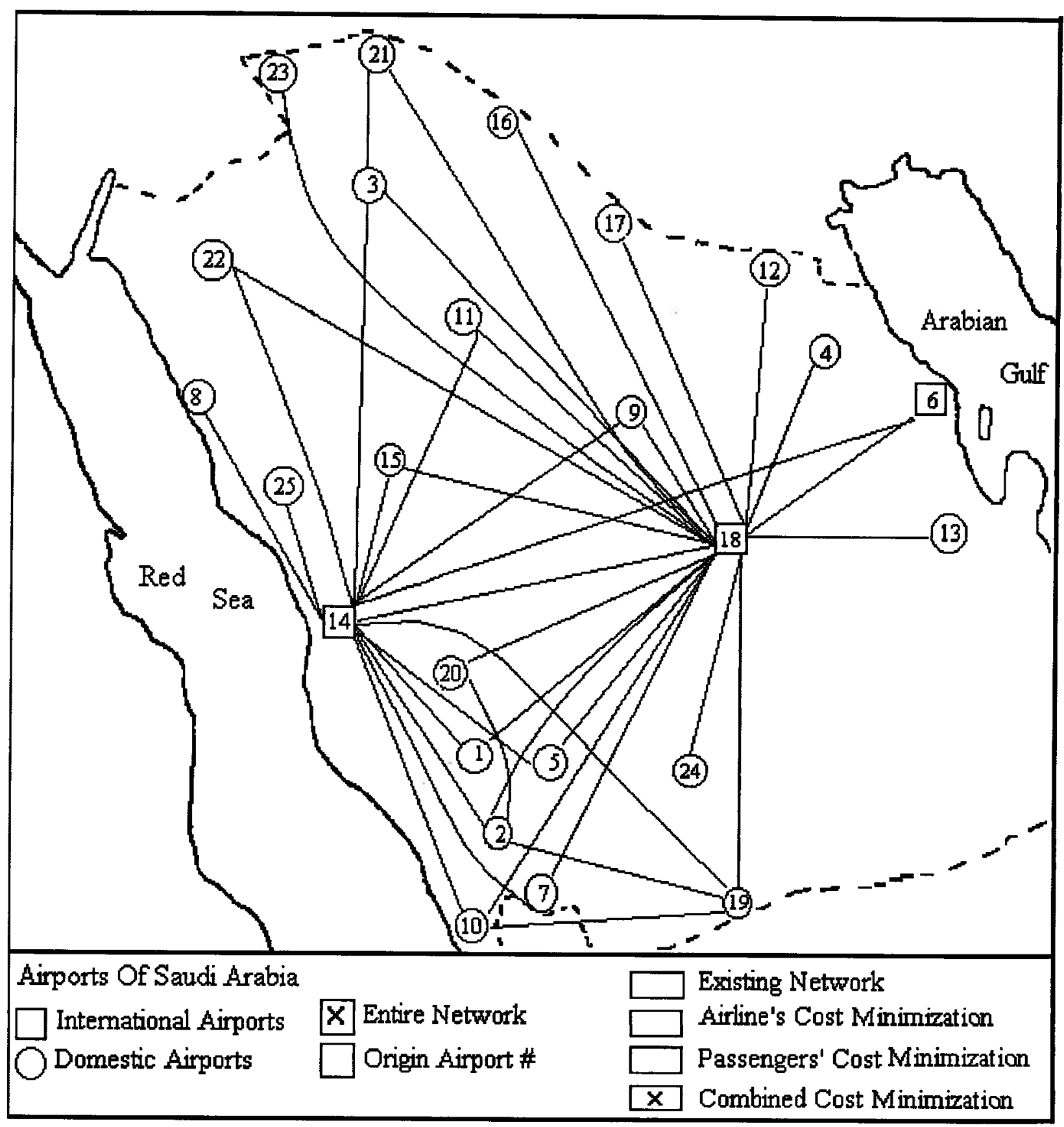

Figure 5. 8: Efficient Combined Cost Network 


\subsubsection{Efficient Airline's Cost Network}

This new network has a total of 172 routes connecting 172 city pairs of origins and destinations. The solution file of this network (see appendix C) indicates that the number of routes that include a transfer has increased from 32 routes in the existing network to 66 routes in this network. Consequently, the total number of direct routes has been reduced from 140 in the existing network to 106 in this network. This happened as a result of the traffic consolidation process and the abandonment of the most expensive point to point connection routes where traffic volumes did not justify direct connections. Consequently, load factors in this network are higher than those in the existing network. Figure 5.9 shows the estimated load factor distribution throughout the network if only the minimum number of flights is operated to meet all travel demands. The figure indicates that links with load factors under $40 \%$ have

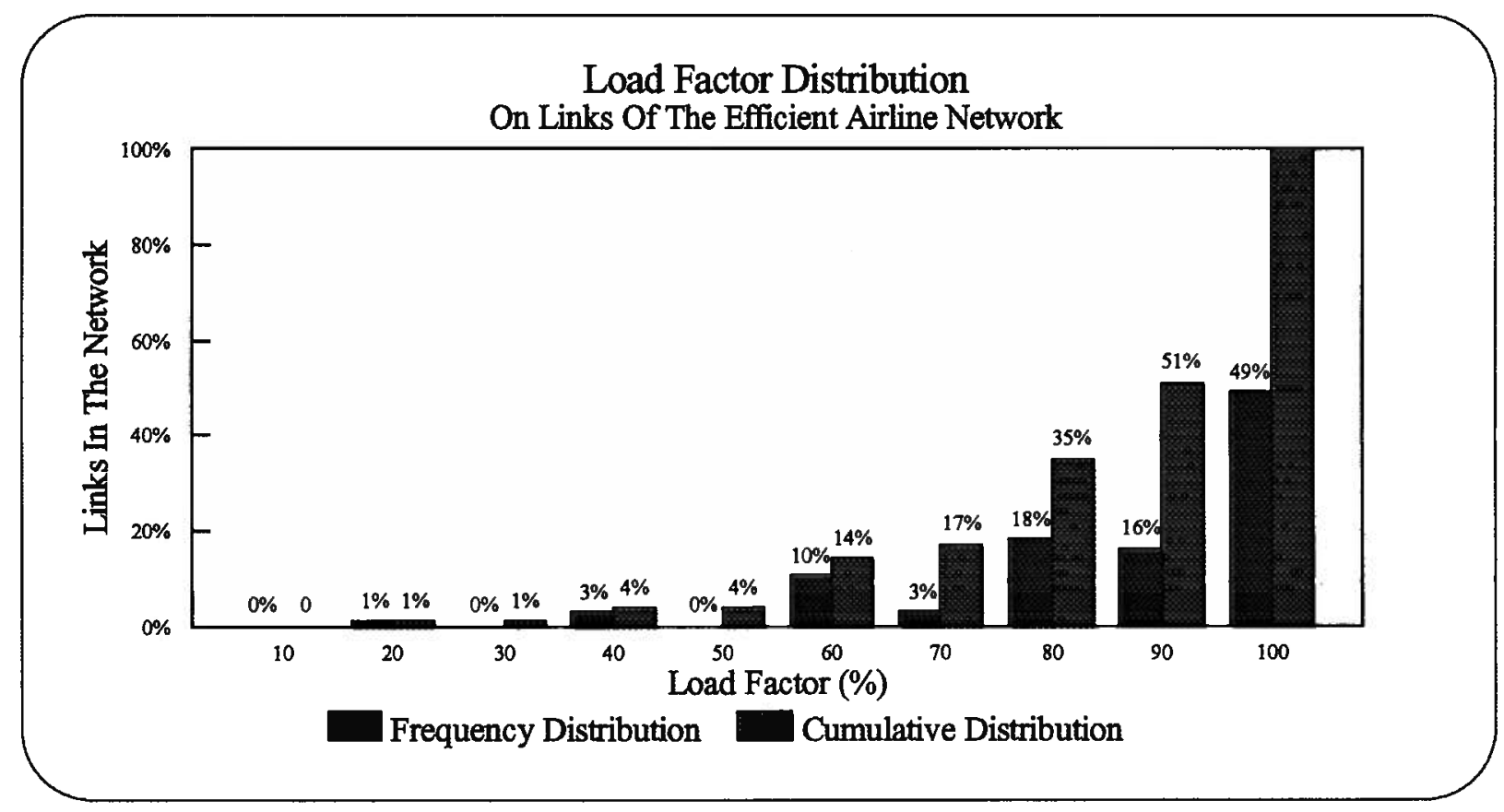

Figure 5. 9 : Estimated Load Factor Distribution In Efficient Airline Network 
decreased from $29 \%$ in the existing network to only $4 \%$ in this network. Also, the fraction of links with load factors higher than $80 \%$ has increased from $58 \%$ in the existing network to $83 \%$ in this network.

Traffic consolidation has also resulted in reducing the total number of required flights. The analysis file of this network (see appendix D) indicates that a total of 745 flights per week are required to serve all travel demands. Links with low traffic volumes were removed and their flights were cancelled. These low traffic volumes were then re-routed on links that has higher traffic demands. This re-routing process has increased the frequency of flights on the majority of links in this network. Figure 5.10 shows the estimated number of flights distribution throughout the network. The figure shows that the fraction of links with one

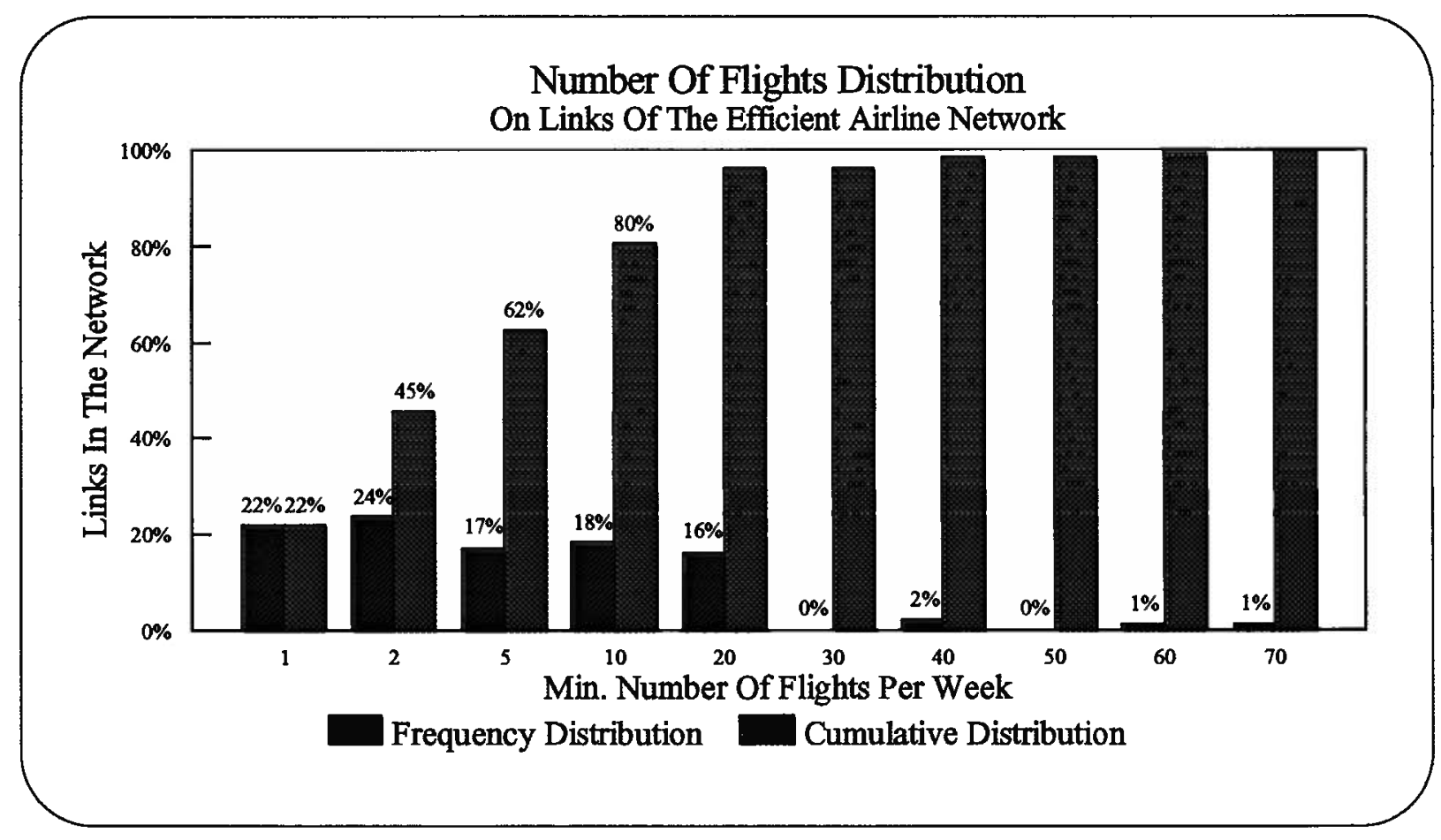

Figure 5. 10: Estimated Number Of Flights Distribution In Efficient Airline Network 
weekly flight has been reduced from $38 \%$ in the existing network to $22 \%$ in this network. The figure also indicates that $38 \%$ of the links in this network can be served by more than 5 weekly flights. This clearly shows the effects of the shape of the network on airline operations and passengers service. By adopting this network, the airline can reduce the total number of flights and simultaneously improve passenger's service by re-routing low traffic volumes on links that has higher flight frequency.

Higher flight frequencies resulted in reducing the schedule delay in the network. By adapting this network structure, the total schedule delay can be reduced by $42 \%$ from 3736 hours per week in the existing network to 2160 hours per week in this network. Figure 5.11 shows the estimated schedule delay distribution throughout the network. The figure indicates that the fraction of links that have schedule delay of 50 hours per week has been reduced from $38 \%$ in the existing network to $22 \%$ in this network. The figure also indicates that $42 \%$ of the routes in the network can experience schedule delays up to 10 hours per week. 


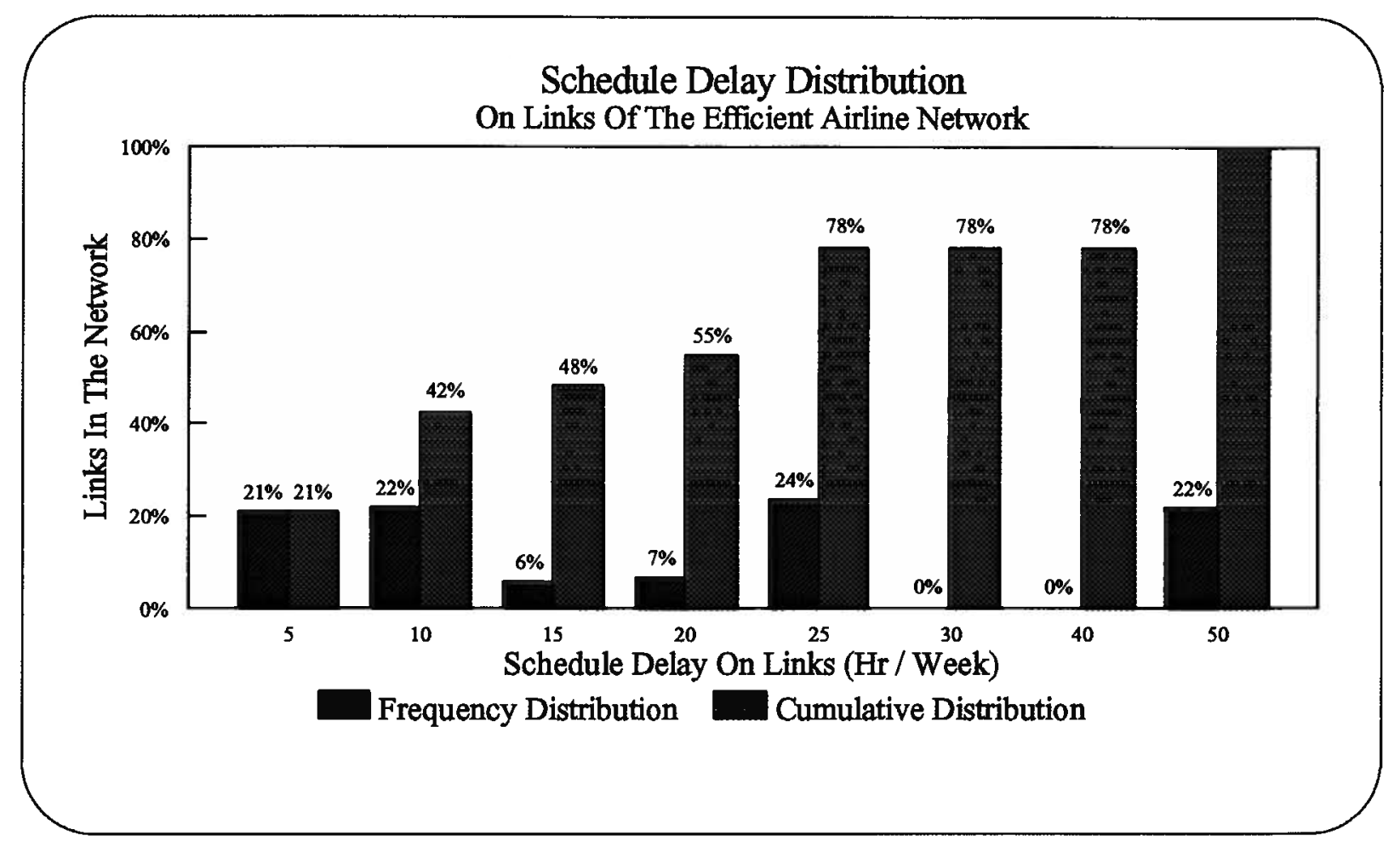

Figure 5.11 : Estimated Schedule Delay Distribution In Efficient Airline Network

Finally, the abandonment of routes with low traffic volumes, the selection of the least costly routes, and the subsequent reduction of the number of required flights can lead to reductions in airline operating cost. By adopting this network, the airline can reduce its current estimated operating cost from 33.0 millions SR per week to 32.4 millions SR. Figure 5. 12 shows the estimated distribution of the estimated cost in terms of the operating cost per passenger-mile. The figure clearly reflects the abandonment of the most costly routes. The highest cost per passenger-mile in this network is 4.5 SR and it can only be found on one percent of the links in the network. 


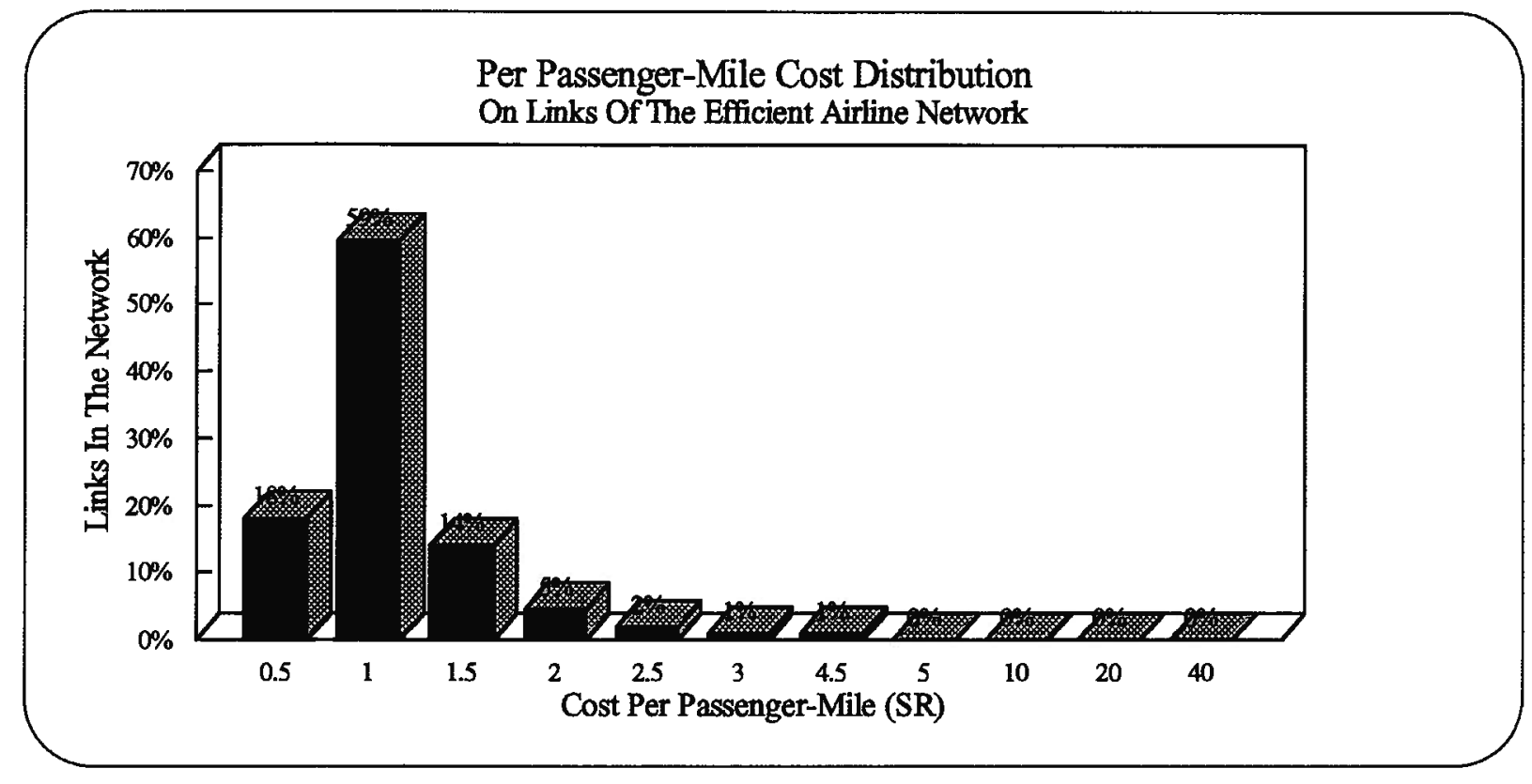

Figure 5. 12 : Estimated Cost Per Passenger-Mile Distribution In Efficient Airline Network

To summarize, the algorithms has produced a network that reduces the airline operating cost by eliminating the most costly direct connection routes and re-routing low traffic volumes through the hub airports. Jeddah and Riyadh airports were identified as the major and most dominant hubs in this new network because of their huge ability to generate and attract traffic. Consequently, the total number of required flights has been reduced and at the same time flight frequencies on the remaining transfer routes has increased providing better service to the passengers by reducing their expected schedule delays. Load factors on the majority of routes are higher in this network than those in the existing network. This fact alone clearly indicates that by adopting this network the airline can improves its operational efficiency and reduces its operating cost. Hansen and $\mathrm{Kanafani}^{50}$ researched the effects of load factors on the operating cost and they have clearly stated " If hubbing resulted in higher load factors or higher average aircraft size, it would also result in lower costs ". Their conclusion is important since it will help in explaining the changes in airline operation cost according to the changes in load factors. 


\subsubsection{Efficient Passenger's Cost Network}

This network was particularly designed to minimize the total travel cost of passengers. This travel cost includes elements such as the ticket price, the cost associated with travel time, and the cost associated with schedule delay. The ticket price between any origin and destination airports was assumed to remain constant regardless of the trip route. Hence only the remaining two cost elements were reduced in this network. This was accomplished by selecting the routes that minimize the total travel time and the schedule delay time. Travel time can be calculated in many ways. One way is to consider the door to door time. In this study however, only the travel time components that relate to the air mode is considered. These time components can includes elements such as check-in time, waiting time, block time, and transfer time. But since the first two elements can not be affected by the chosen travel route, only the last two time elements were used and minimized in this network.

The origin destination matrix presented in appendix (A) clearly shows that travel demand levels have wide variations between the different origin destinations pairs. This can cause wide variations in the required flight frequencies between the different airport pairs. In return, this made the schedule delay time the most dominant time element. Hence this network is mostly comprised of routes that reduces the schedule delay time. The network has a total of 172 routes. 67 of these routes are direct routes and 105 are transfer routes.

As indicated by the network solution file presented in appendix (C) and the map of this network presented in figure 5.7, the majority of these routes are transfer routes that send traffic through Jeddah (airport number 14) or Riyadh (airport number 18) airports. These two airports 
are the major traffic generators in the network, and therefore they generate the largest flight frequencies. For this reason the majority of trips are routed through these two airports.

Figure 5.13 shows the estimated load factor distribution throughout the network if only the minimum number of flights is operated to serve all demands. A comparison between figures 5.2 and 5.13 indicates that this network has higher load factors than the existing network on approximately $36 \%$ of the routes. This fact alone shows that by adopting this network traffic can be consolidated on some routes. At the same time, the figure indicates that $10 \%$ of the routes have load factors under $20 \%$. Comparing this fact with figure 5. 9 which indicated that only $4 \%$ of the routes in the efficient airline network has load factors under $50 \%$ clearly indicates that the degree of traffic consolidation in this network is not as large as that in the efficient airline network. This is observation is expected since this network is intended to reduce the passenger travel cost and not the airline operating cost. 


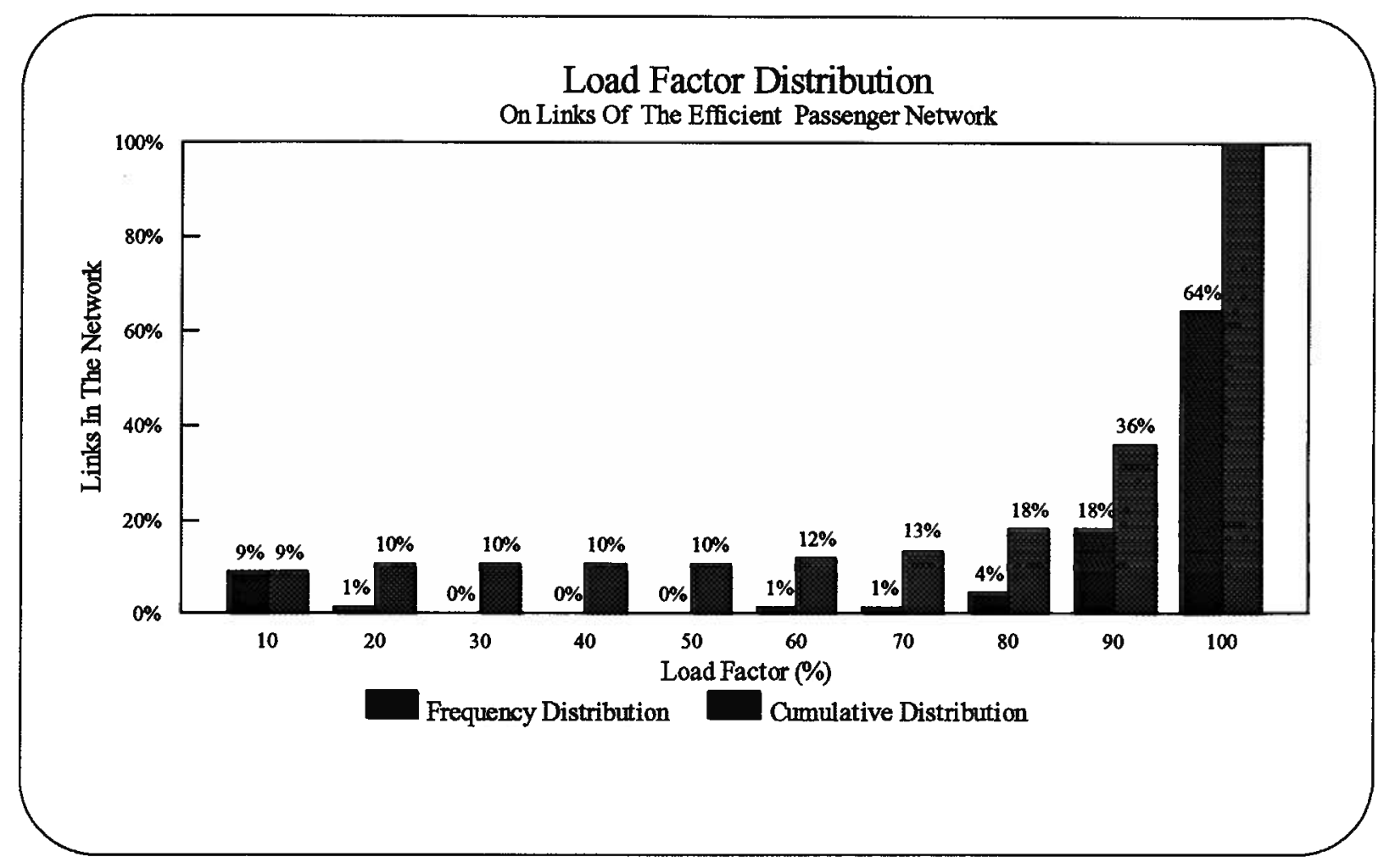

Figure 5. 13 : Estimated Load Factor Distribution In The Efficient Passenger Network

Since this network was designed to minimize the cost of travel of passengers, the majority of its routes has the highest flight frequencies as compared to the previous two networks. Figure 5.14 shows the estimated number of flights distribution throughout the network. The figure indicates that the fraction of links with one flight per week is reduced to only $12 \%$ as compared to $39 \%$ and $22 \%$ in the previous two networks. The figure also indicates that $64 \%$ of the links in the network can be served by more than 5 weekly flights. To provide these flight frequencies it is estimated that this network would require a minimum of 766 flights per week to be operated to serve all traffic demands. 


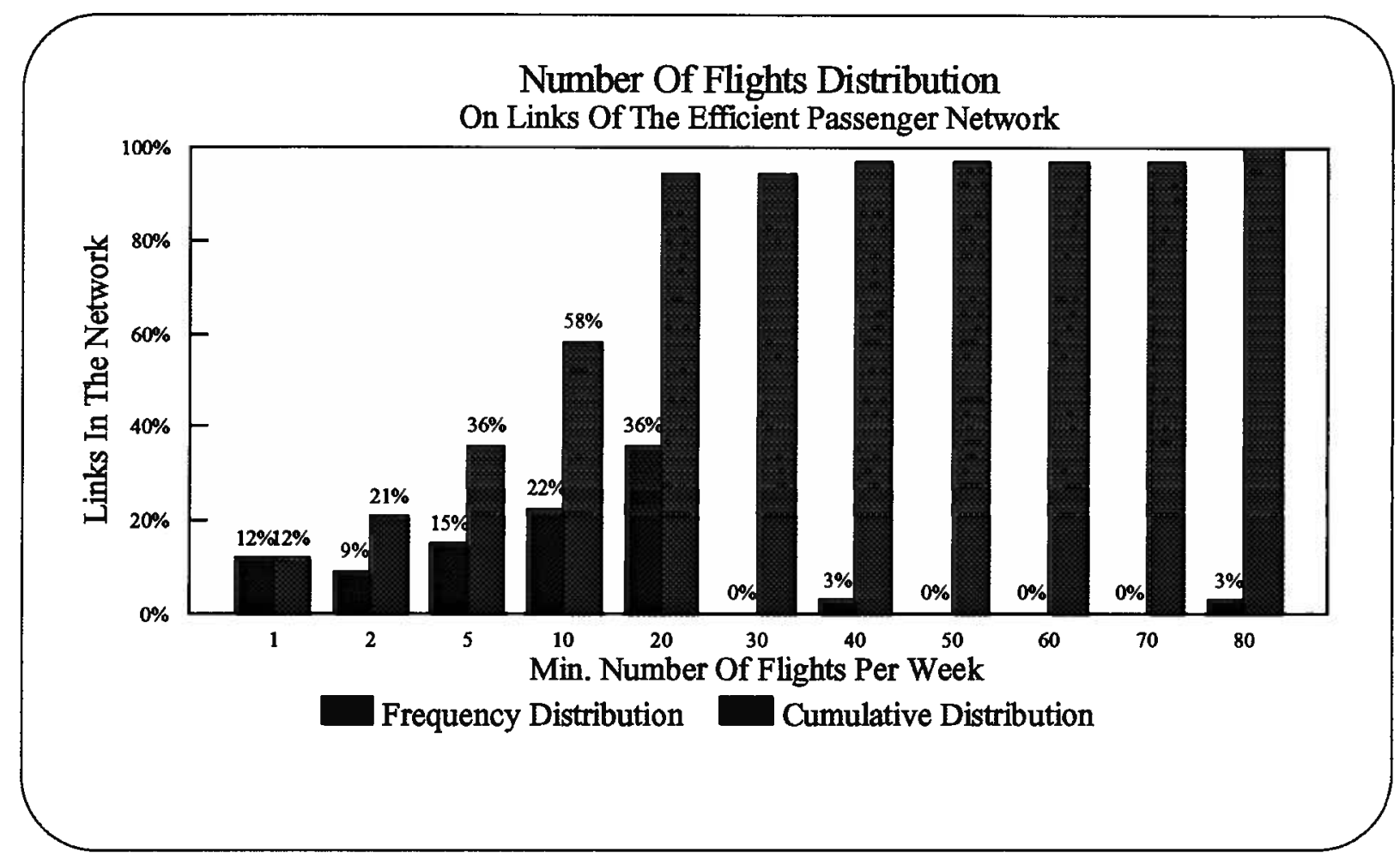

Figure 5. 14 : Estimated Number Of Flights Distribution In The Efficient Passenger's Network

Routing traffic on links with the highest flight frequencies has resulted in huge reduction in schedule delays in this network. By adapting this network, the total schedule delay can be reduced by $77 \%$ from 3736 hours per week in the existing network to 843 hours per week in this network. Figure 5.15 shows the estimated schedule delay distribution throughout the network. The figure indicates that only $12 \%$ of the links in this network are expected to experience schedule delay that ranges between 25 and 50 hours per week. The figure also indicates that $43 \%$ of the routes can have schedule delays under 5 hours per week. This clearly indicates that this network would provide better levels of service to the passenger than the two previous networks. 


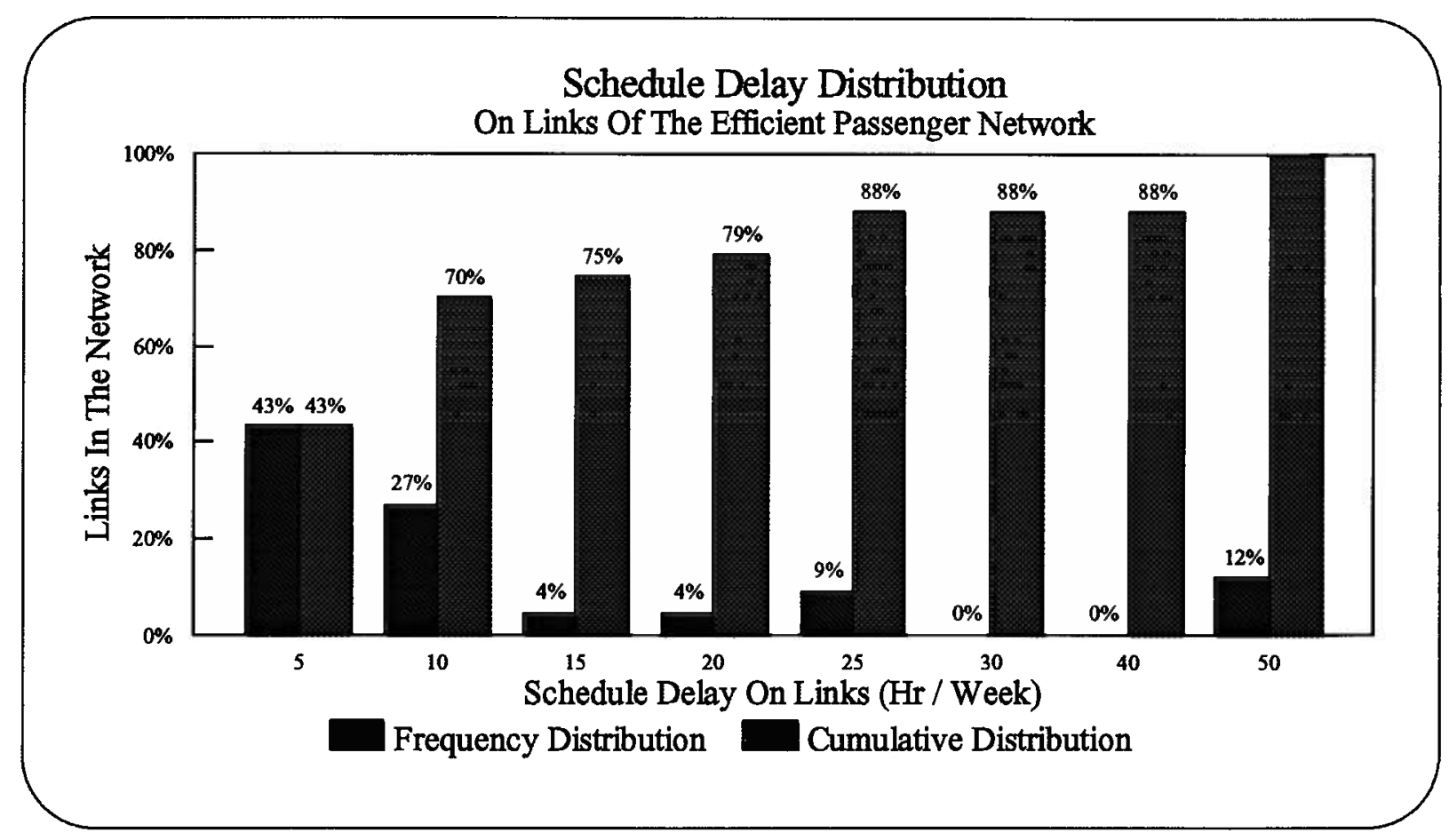

Figure 5. 15 : Estimated Schedule Delay Distribution In The Efficient Passenger's Network

Finally, figure 5.16 shows the estimated distribution of the cost of operations in terms of the operating cost per passenger-mile. A casual examination of this figure alone can be misleading because it indicates that the majority of links in this network has lower per passenger-mile costs than those links in the existing network. This might lead the reader into thinking that this network would be less costly to operate than the existing network. However, operating this network will be more costly to the airline. Airline operating cost is estimated to increase from 33.0 millions to 34.4 millions SR per week if this network is adapted. This increase in cost can be explained by comparing figures 5.2 and 5.13. The comparison shows that this network has higher load factors on approximately one third of its routes than the existing network. However, since these higher load factors were limited to a minority of routes in this network and the routes were selected to minimize the travel cost of the passengers, airline operating cost of this network is higher than that of the existing network. Also, the 
routes in this network are not necessarily the least costly to operate on, they are however the ones that provide the least travel and schedule delay times. This is so because the objective of developing this network is the reduction of the travel cost of the passengers.

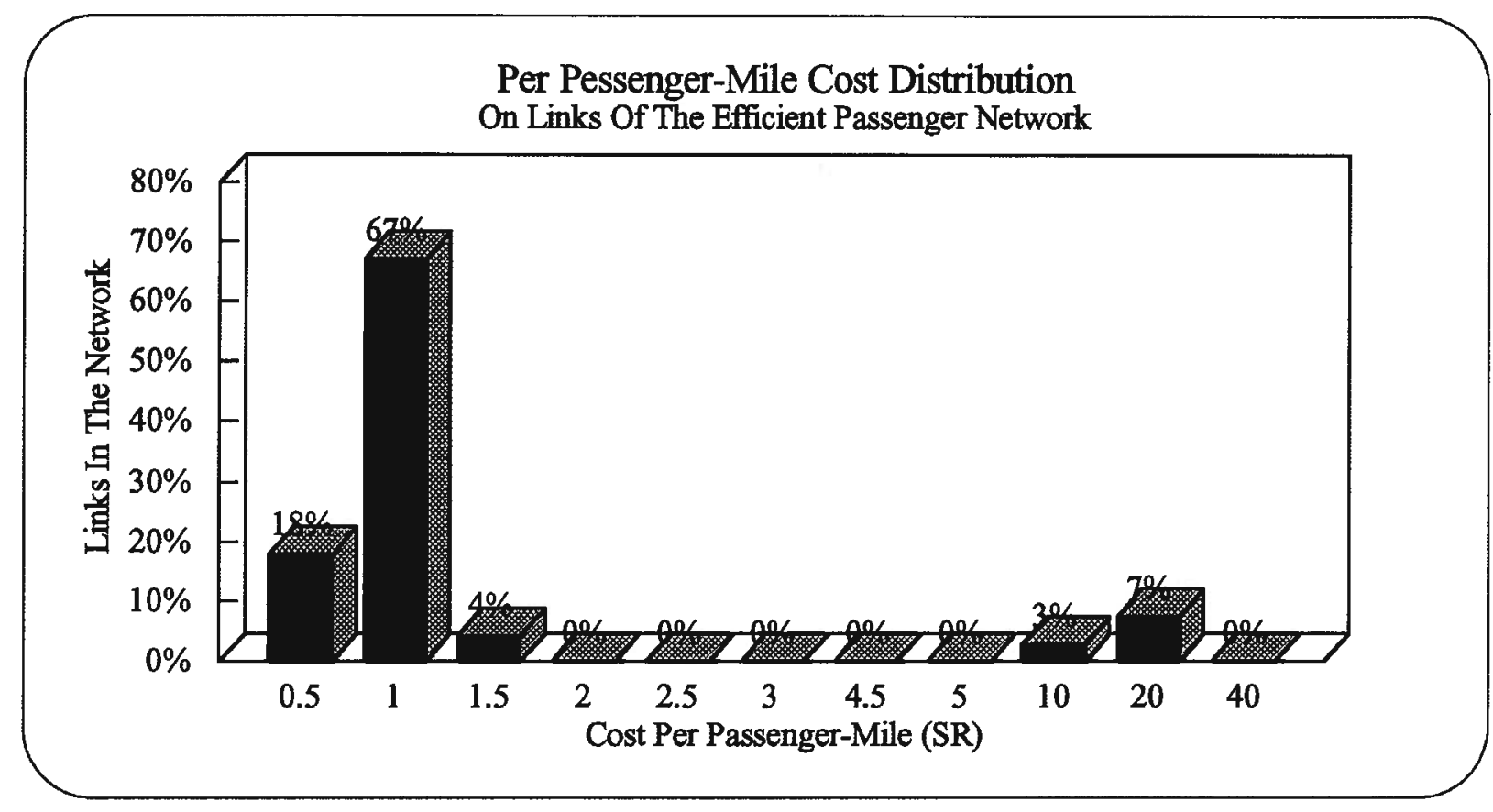

Figure 5. 16: Estimated Cost Per Passenger-Mile Distribution In EfficientPassenger' Network

To summarize, this network provide better service levels to the travellers by routing them on routes that have high flight frequencies. High flight frequencies minimizes schedule delays which in return reduces the passengers cost of travel. Traffic is consolidated on some routes in this network. This is clearly indicated by comparing figure 5.13 with figure 5.2 . The comparison shows that this network has higher load factors on approximately one third of its routes than the existing network. However, since these higher load factors were limited and the routes were selected to minimize the travel cost of the passengers, airline operating cost of this network is higher than that of the existing network. 


\subsubsection{Efficient Combined Cost Network}

This network was specifically designed to minimize the total cost of both the airline and the passengers. This has resulted in developing a network that is comprised of a collection of links from the efficient airline and passengers networks. The solution file provided in the appendix shows that this network has 76 direct routes and 96 routes that include a transfer either through Jeddah or Riyadh airports.

Load factors in this network are higher than any other network indicating a high level of traffic consolidation on its routes. Figure 5.17 shows the estimated load factor distribution throughout the network if only the minimum number of flights are operated to serve all travel demands. The figure shows that the majority of routes $(64 \%)$ will have load factors that ranges

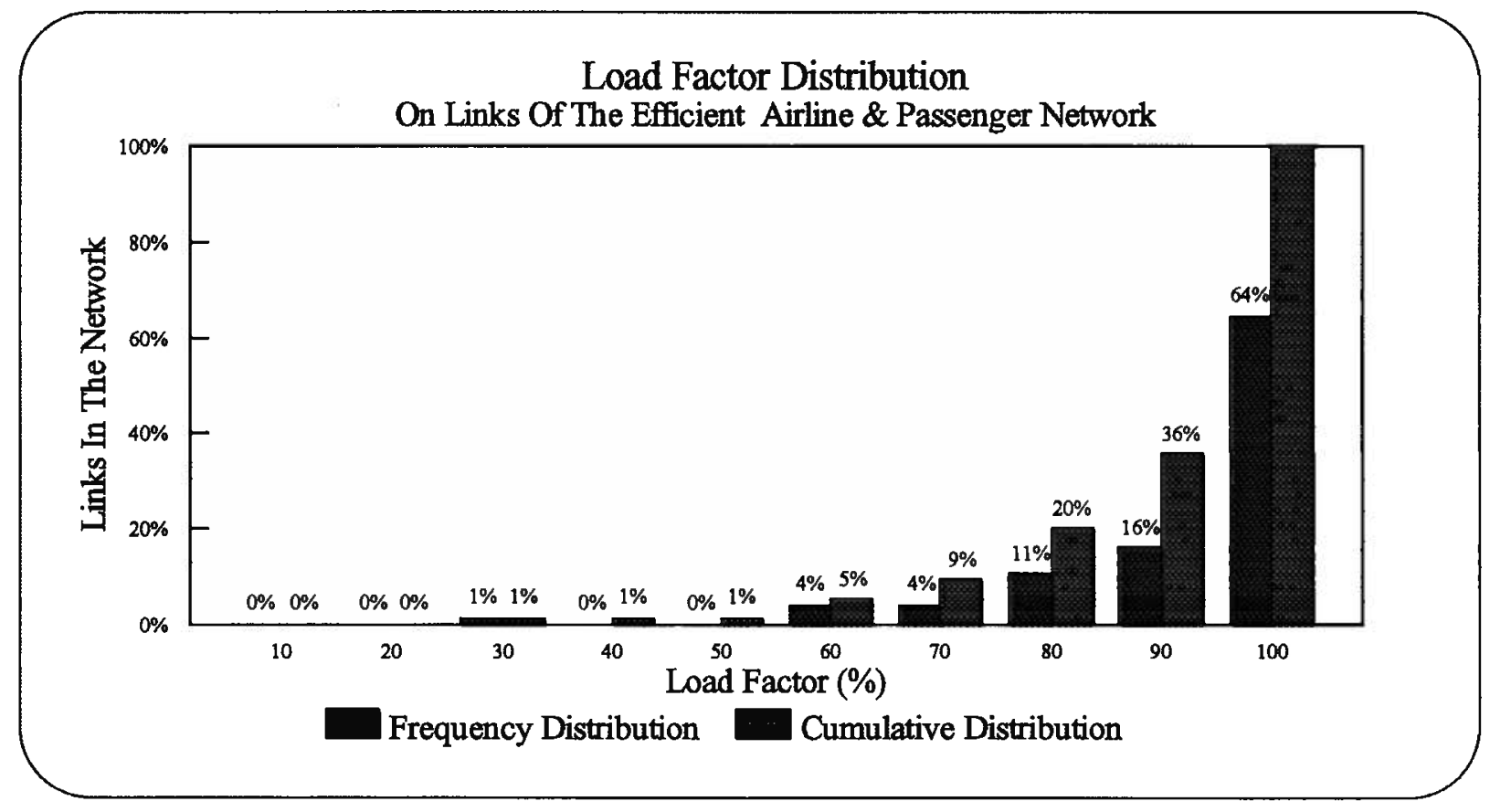

Figure 5. 17 : Estimated Load Factor Distribution In The Efficient Combined Cost Network 
between 90 1nd 100 percent. The figure also indicates that only $1 \%$ of the links in the network have the lowest load factor of $30 \%$. $95 \%$ of the links will have load factors higher than $60 \%$.

Traffic consolidation resulted in reducing the number of direct links in the network and the number of required flights. It is estimated that a total of 744 flights per week will be required to serve all travel demands in this network. Figure 5.18 shows the estimated number of flight distribution throughout the network. The figure indicates that the fraction of links with one weekly flight has been significantly reduced from $38 \%$ in the existing network to $3 \%$ in this network. It is worth to note that flight frequencies of the majority of links are higher in this network than the frequencies of the existing network even though the total number of required flights is lower in this network.

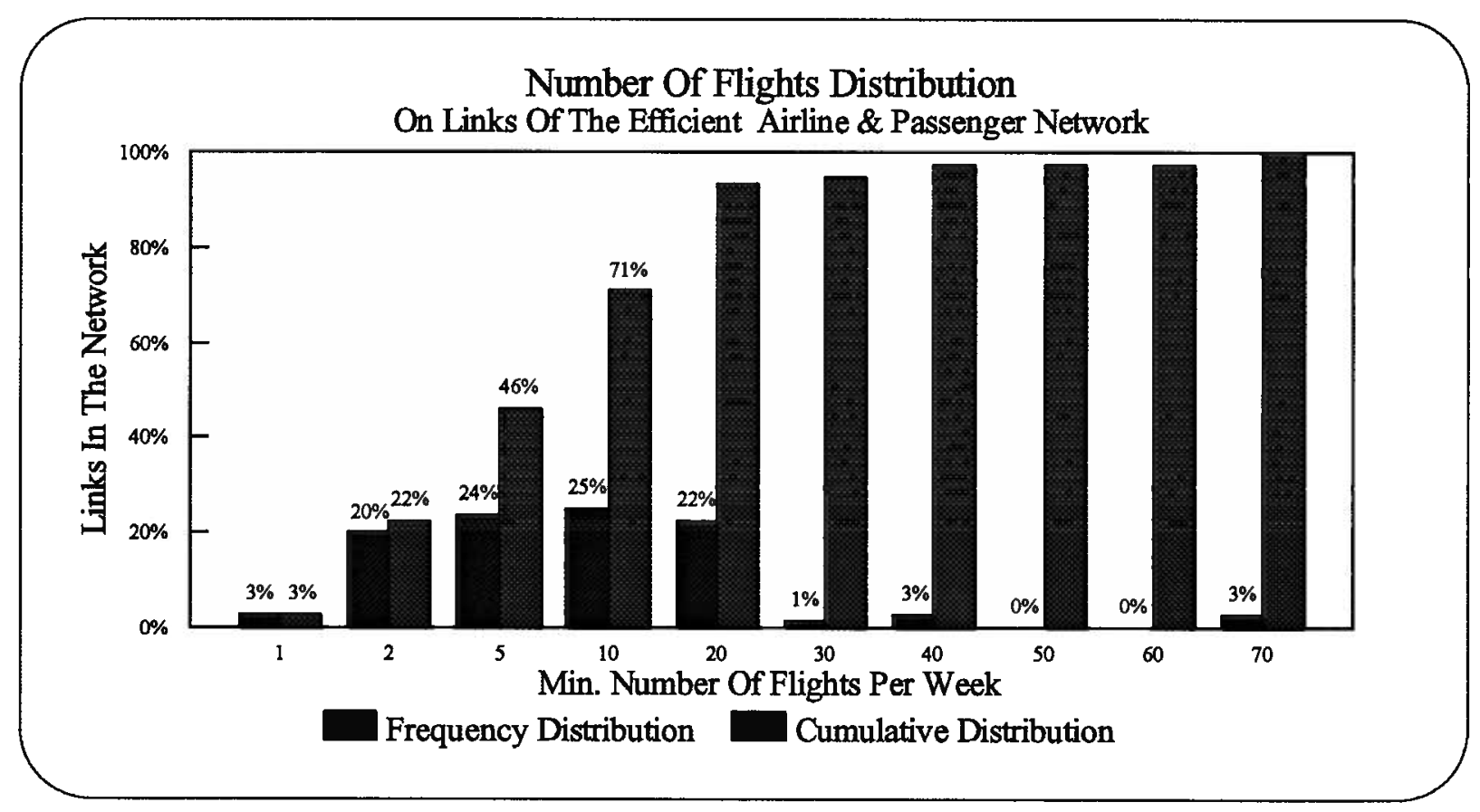

Figure 5. 18 : Estimated Number Of Flights Distribution In Efficient Combined Cost Network 
Higher flight frequencies resulted in reducing the schedule delays and improved the service levels. By adapting this network structure, the total schedule delay can be reduced by $76 \%$ from 3736 hours per week in the existing network to 881 hours per week in this network. Figure 5.19 shows the estimated schedule delay distribution throughout the network. The figure indicates that the fraction of links that have schedule delay of 50 hours per week has been reduced from $38 \%$ in the existing network to $3 \%$. The figure also indicates that $62 \%$ of the routes in the network can experience an average schedule delays up to 10 hours per week.

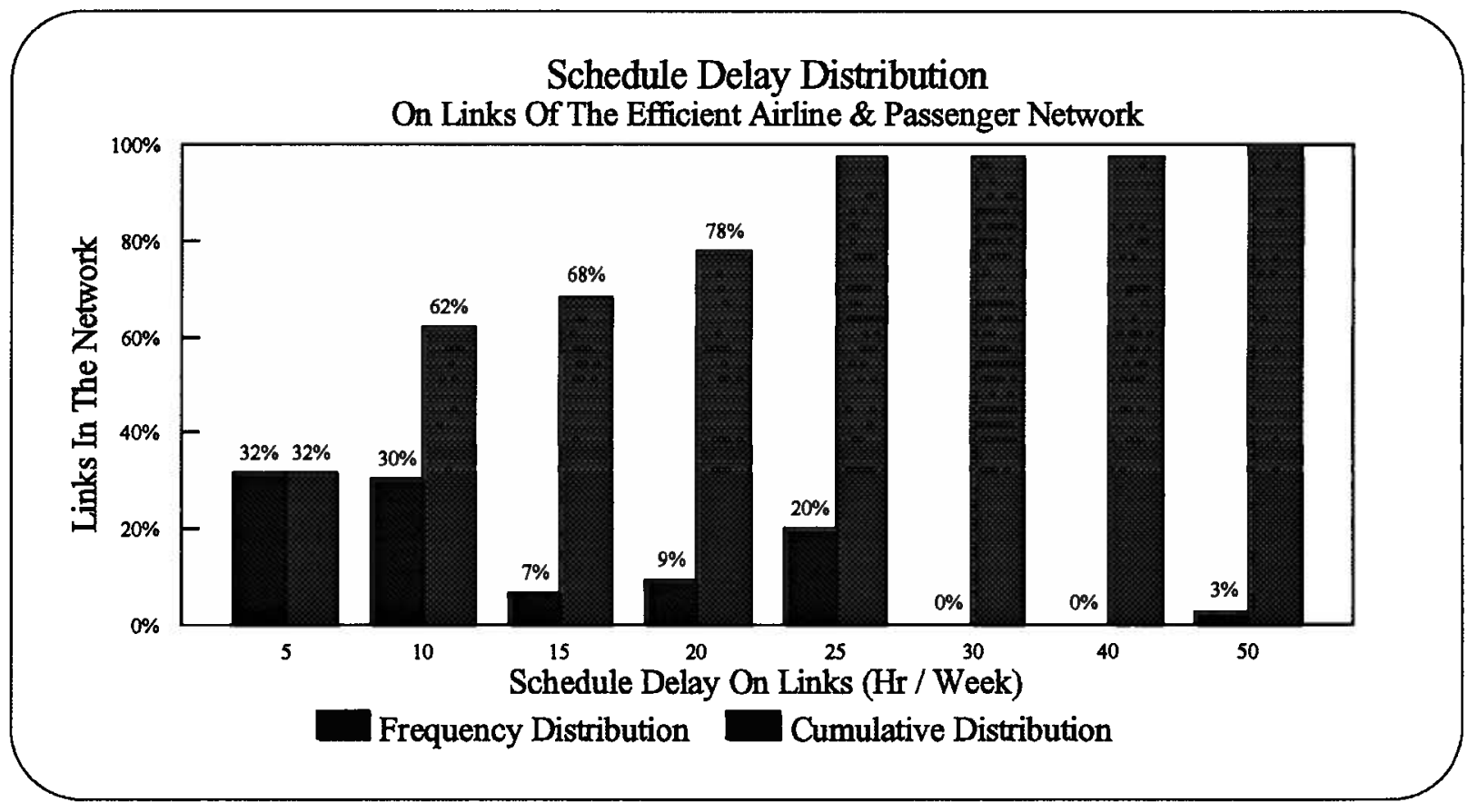

Figure 5. 19 : Estimated Schedule Delay Distribution In The Efficient Combined Cost Network

Traffic consolidations and reduction in the number of flights have reduced the total cost of airline operations. By adapting this network, the airline can reduce its current estimated cost of operations from 33.0 millions SR per week to 32.8 millions SR per week. Although this network has the highest degree of traffic consolidations as indicated by its load factors distribution in figure 5.17, its savings in operation cost is not as high as those of the efficient 
airline network. This is so because some of the routes in this network are not necessarily the least costly to operate on. These routes were included in the network to particularly reduce the travel cost of the passengers. In essence a trade off between the two costs have taken place to reduce the total sum of the two costs. To understand this trade off it is helpful to remember that the objective of developing this network is to minimize the total costs of both the airline and the passengers. Figure 5.20 shows the estimated distribution of the airline operating cost in terms of the operating cost per passenger-mile. A casual comparison of this figure with figure 5.12 can be misleading. This is so because this figure indicates that the majority of the links in this network have lower per passenger-mile cost than those links in the efficient airline network. Therefore, this network should have lower total cost of operations than the efficient airline network. However, this is not the case, this network has higher cost of operations. The higher cost can be explained by the absolute numbers of links and their operating costs in each network. This network has less number of links that have low operating costs than the other network. For example, the efficient airline network has 63 links with an operational cost of 1.0 SR per passenger mile. This network has only 52 links that falls in the same cost category. 


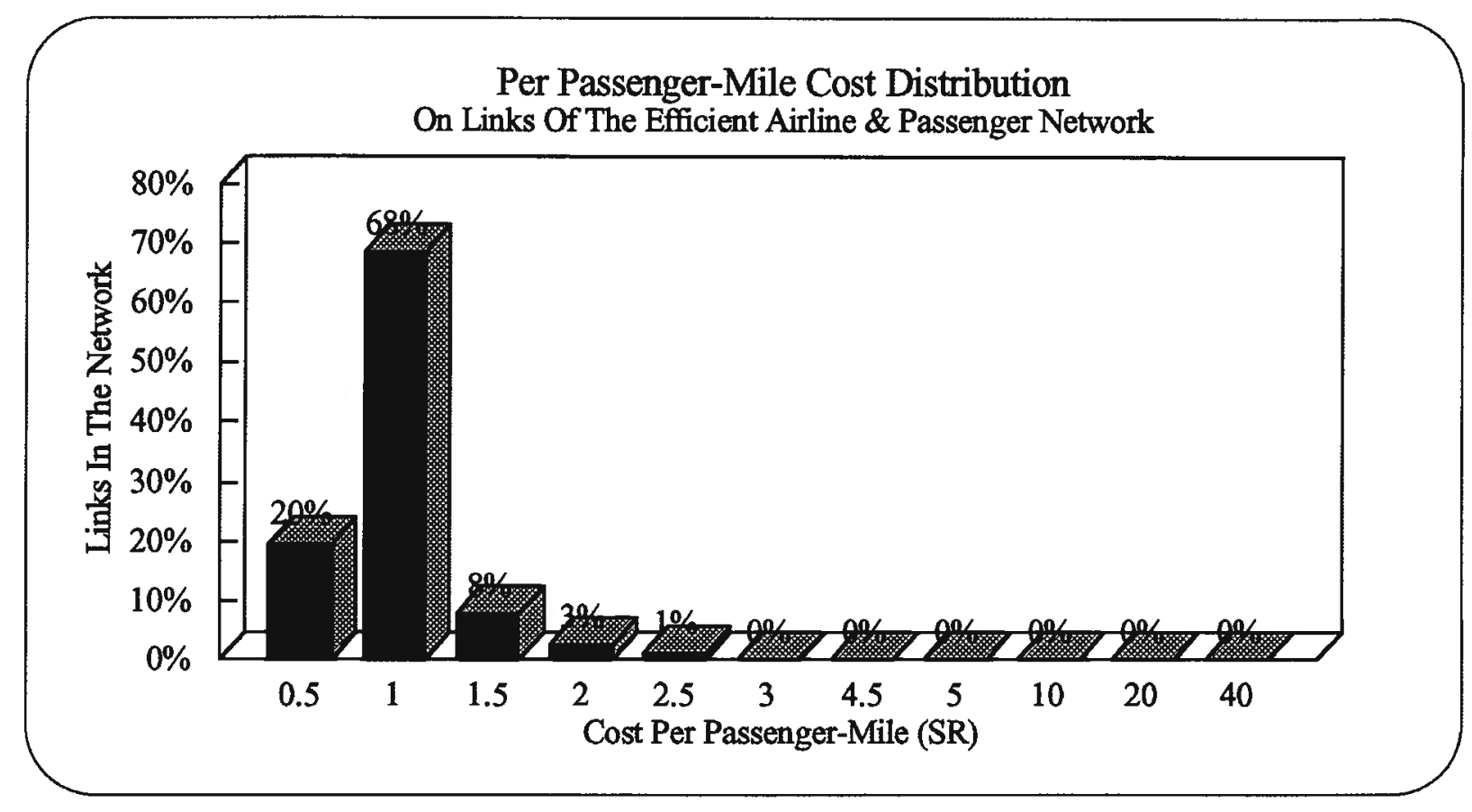

Figure 5. 20 : Estimated Cost Per Passenger-Mile Distribution

In The Efficient Combined Cost Network

To summarize, the algorithm successfully produced a network that fulfilled its design objective by reducing the total cost of travel for both the airline and the passenger. By adapting this network the airline can save 0.2 million SR per week as compared to their current operations. Passengers' savings are much higher, this network will reduce the total schedule delays and its associated cost by $76 \%$.

\subsubsection{Summary}

The above networks analysis clearly indicates that the developed routing algorithms have successfully created networks that meet their specific design objectives. The new networks reduce both the airline operating cost, and passengers' travel cost. Any of these 
networks should not be considered or looked at in isolation as a final network design by it self. The three network designs must be viewed as a decision making tool that helps the decision maker. To select a network for operations, all network designs must be evaluated against a set of predetermined goals and objectives that relates to airline operations and passengers' service levels. Finally, table 5.1 offers comparisons between key attributes of the different networks.

\begin{tabular}{||l|c|c|c|c||}
\hline \hline & $\begin{array}{c}\text { Existing } \\
\text { Network }\end{array}$ & $\begin{array}{c}\text { Airline } \\
\text { Network }\end{array}$ & $\begin{array}{c}\text { Passengers } \\
\text { Network }\end{array}$ & $\begin{array}{c}\text { Combined } \\
\text { Network }\end{array}$ \\
\hline Number of Direct Routes & 140 & 106 & 67 & 76 \\
Number of Transfer Routes & 32 & 66 & 105 & 96 \\
\hline Average Load Factor (\%) & 66 & 83 & 84 & 89 \\
\hline Number of Flights & 778 & 745 & 766 & 744 \\
\hline Average Sched. Delay (hr/week) & 26.96 & 20.38 & 12.60 & 11.60 \\
Total Sched. Delay (hr/week) & 3736 & 2160 & 843 & 881 \\
\hline Average Operating Cost (SR/Passenger - mi ) & 2.54 & 0.84 & 1.58 & 0.71 \\
Total Operating Cost (Million SR/week) & 33.0 & 32.4 & 34.4 & 32.8 \\
\hline Total Passenger-miles Flown & $54,875,896$ & $55,231,064$ & $59,562,624$ & $56,543,592$ \\
\hline
\end{tabular}

Table 5.1 : Attributes of Existing And Design Networks

It is interesting to note in this table that the average operating cost per passenger-mile of the efficient combined network ( $0.71 \mathrm{SR})$ is lower than that of the efficient airline network $(0.84$ SR). Hence, one might think that the combined network is less costly to operate than the efficient airline network but, this is not the case. In fact, the total operating cost of the efficient airline network is estimated to be 32.4 Million SR/week while the total operating cost of the combined network is estimated to be 32.8 Million SR/week. The lower average operating cost per passenger-mile of the combined network can be explained by the fact that the size of this network in terms of the passenger-miles flown $(56,543,592)$ is larger than the size of the efficient airline network $(55,231,064)$. 


\section{CONCLUSIONS}

This thesis developed efficient routing algorithms for routing air traffic in situations where demand levels are sparse and spread over large geographical areas. Three algorithms were developed and used to create three efficient network structures. The networks were designed to attain specific objectives. The first network minimized the total cost of airline operations. The minimum airline cost network consolidated traffic demands at a few ideal hub locations, and reduced the minimum number of required flights that meet all traffic demands. The second network minimized the cost of travel of the passengers by reducing their travel and schedule delay times. In this network trips were assigned to the links with the least travel and schedule delay times. The third network minimized the total combined cost of both the airline and the passengers. This was done by consolidating the traffic at a few ideal hub locations to minimize the airline unit costs of operations, and by assigning the trips to the routes that have the least schedule delays, and travel times.

The case study showed that all algorithms have successfully achieved their design objectives, and made it possible to explore the influence of the structural shape of air transport networks on the operational cost of the airline, the passengers cost of travel, and the level of service. Also, in the analysis part of the study, the following conclusions were reached:

1. The attributes of air transport networks influence both the passengers' travel cost and the airline operating cost. The total cost and network structure are not independent. 
Hence, air transport networks must be developed in such a fashion that total costs are minimized and travel service within the network maximized.

2. In networks with sparse demand for travel, operating costs are greatly influenced by the shape of the network. Increased direct point to point connectivity in such networks will increase the airline operating cost, reduce load factors, and increase schedule delays.

3. In sparse demand networks, the consolidations of traffic demands through the usage of the hub and spoke routing strategy will increase load factors, increase the frequency of service, reduce schedule delays, and minimize the airline operating cost.

4. The usage of unit costs links the demand distribution throughout the network to the process of creating the network. This will result in the creation of a network that is "demand driven". Hence, if the demand distribution change, the network shape will also change to accommodate the new demand distribution. This is so because any change in the demand distribution will cause a change in the unit costs.

5. The new passengers' network design showed that traffic consolidation alone does not automatically reduces the airline operating cost. In order to do so, the network routes must be selected in such a fashion that minimizes the unit costs of transport.

6. In Saudi Arabia the existing airline network was designed to meet all passengers demands regardless of the sparse volumes. As a result of government policies, the political integration of the country as well as the social well-being and the comfort of 
the population were given greater considerations over the economical operations of the airline. Consequently, all the kingdom's centres of population are directly connected to one another even though demand levels do not necessarily justify direct connection.

7. Figures 6.1 shows the cancelled links of the existing network in the new airline's, passengers', and combined networks. The figure also shows the population concentrations around the different airports in Saudi Arabia. It is interesting to note that the majority of the cancelled links provide direct connections between areas with low population concentrations in the northern part of the country. These low population concentrations explain the cancellations of the direct links in the new networks since such population levels can only generate low traffic demands which result in higher unit costs and low frequency of service on these links. The same figure also shows the added links to the new networks. These links were added as substitutes for some of the deleted links. 


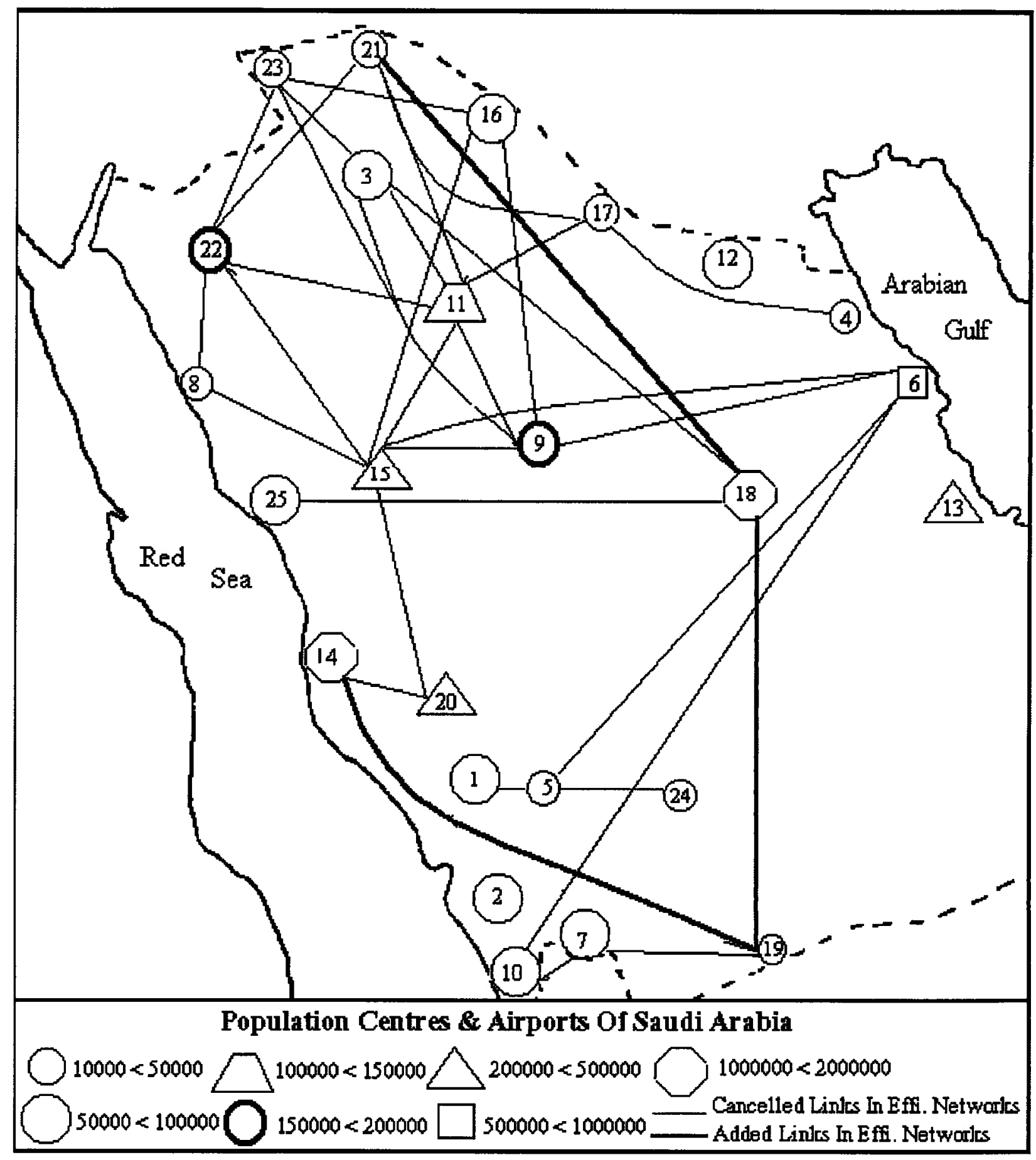

Figure 6.1: Cancelled Links From The Existing Network In The Efficient Networks 


\section{FURTHER RESEARCH}

In this thesis, demand volumes and distributions were treated as static parameters. However, air traffic demand is dynamic and responds to changes in a wide variety of factors including cost of travel, and frequency of service. As previously mentioned, all design networks will improve the frequency of service. These improvements can positively stimulate the demand levels. And since any changes in the demands can alter the unit costs on the various links in the network, the network configuration may change. If demand levels grow substantially, more direct links will be created. However, if the growth is marginal, hubbing will be reinforced. Demand levels do not change quickly, therefore, demand levels and distribution throughout the network should be periodically monitored. The routing algorithms developed in this thesis may be linked to a demand forecasting model to revise the network structure. Also, to further improve the airline operations in Saudi Arabia, a flight scheduling model must be developed and linked to the routing algorithms as can be seen in Figure 7.1.

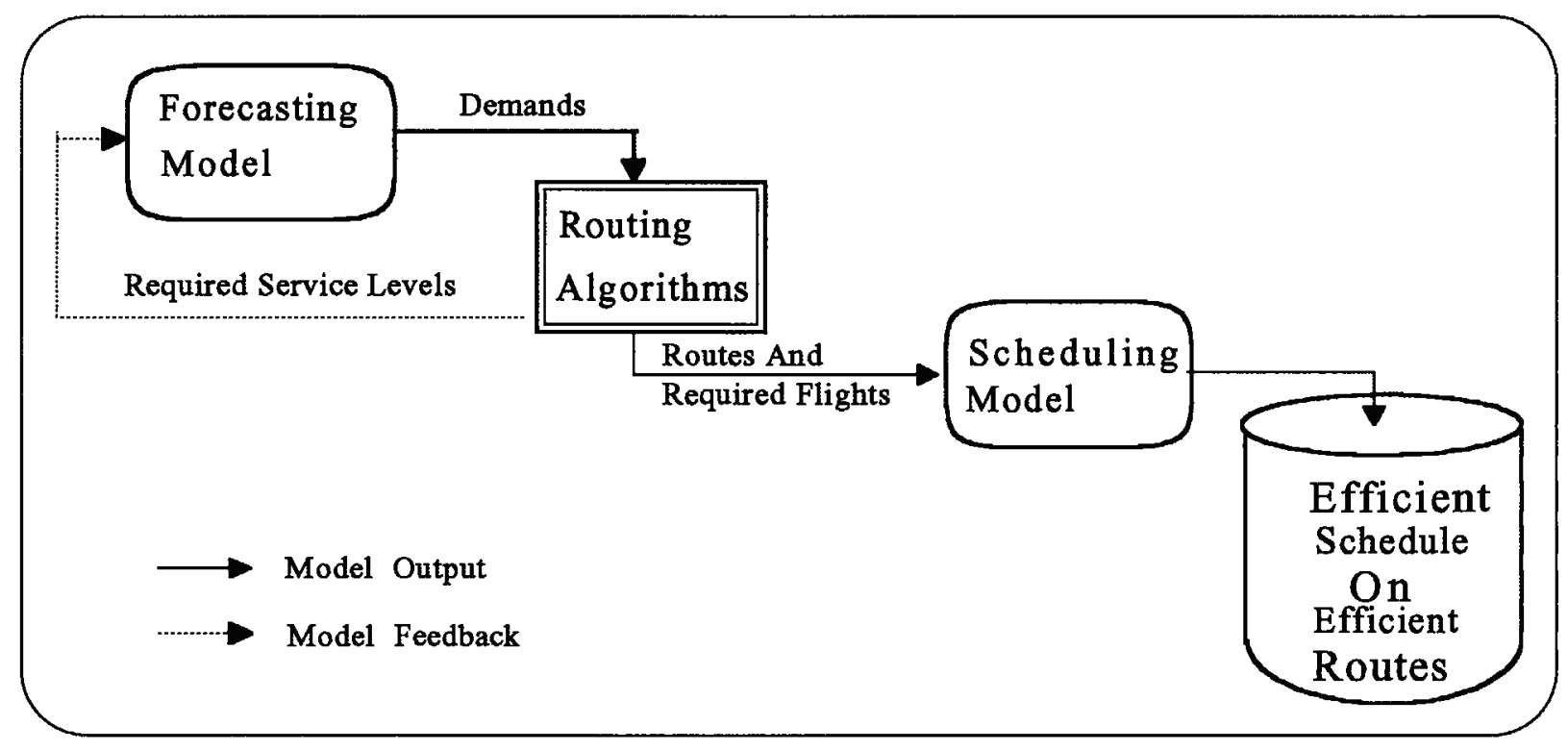

Figure 7.1: Required Models For Efficient Airline Operations 
The developed networks must be refined by refining some of the parameters used in their creation. For example accurate measure of the value of travel time in Saudi Arabia must be determined. This is important because the value of travel time is a western concept, its applicability and its magnitude in a country like Saudi Arabia must be determined for accurately converting time units of schedule delays and travel times into their equivalent monetary values. Also, the full average hourly wage rate was used in this thesis as a measure of the value of travel time. It must be noted that the model is not limited to this value, but this value was used because several studies on the value of air travel time including Gronau's ${ }^{51}$ suggested justifiably the usage of the full wage rate. The common justification in these studies is the notion that travel time is mostly wasted in the sense that it can not be used for conducting any productive work. These studies have also argued that the majority of air travellers earn more money than travellers using other modes of travel. Therefore, air travellers time is argued to be more valuable and thus the full wage rate was used. However, with the development of notebook computers it can be argued that some portion of the travel time can be used for conducting productive work. In this case, the full wage rate is not an accurate measure. Therefore, an accurate measure of the value of air travel time must be determined and used in such a case.

The impact of using smaller air craft size on the shape of the network can be investigated. In this thesis it was assumed that the airline will use the same type of aircraft that it is currently using. It must be noted that the model is not limited to the current aircraft size, and any aircraft size can be used as an input to the model. Using smaller aircraft will definitely change the shape of the design networks simply because different aircraft types have different operating costs which will be reflected on the unit costs of operations on the different links. 


\begin{abstract}
Also, when using smaller aircraft traffic may not need to be consolidated as much as is the case when using larger aircraft. Hence, more direct routes can be established in the network with the usage of smaller aircraft size.
\end{abstract}

This model was particularly developed for routing air traffic in situations where travel demand is sparse and air services are monopolized by one, or a small number of airlines. Its applicability in competitive and large demand situations is not known at this time, but it can be investigated.

Finally, while the developed networks were for Saudi Arabia, the applicability of the algorithms to other areas with similarly sparse demands such as Australia, Northern Canada could be investigated. 


\section{REFERENCES}

Yaw Jeng, Chawn., "An Idealized Model for Understanding Impacts of Key Network Parameters on Airline Routing"., Transportation Research Record 1158., Transportation Reseach Board., National Research Council., Wash, D.C., 1988.

Schwieterman, Joseph P., "Airline Routes and Metropolitan Areas : Changing Access To Non-Stop Service Under Deregulation"., Transportation Research Record 1161., Transportation Reseach Board, National Research Council, Wash, D.C., 1988.

Kanafani, Adib., and Hansen, Mark., "Hubbing And airline Costs", Institute Of Transportation Studies., University Of California., Berkeley., Research Report UCB-ITS-RR-85-12., August 1985.

Shaw, Stephen., Airline Marketing And Management., Pitman Publishing., 1985.

Baily, Elizabeth E., Graham, David R., and Kaplan, Daniel P., Deregulating The Airlines., 1985.

Higgins, R. G., and Toh, R. S., "The Impact of Hub and Spoke Network Centralization and Route Monopoly on Domestic Airline Profitability"., Transportation Journal Summer 1985.

Wheeler, Colin F., "Strategies for Maximizing the Profitability of Airline Hub and spoke Networks"., Transportation Research Record 1214., Transportation Research Record 1158., Transportation Reseach Board., National Research Council., Wash, D.C., 1989.

See Wheeler., Reference 7 Above.

Morrison, S., and Winston, C., The Economic Effects of airline Deregulation, Brookngs Institution., Washington, D. C., 1986., P. 18.

See Wheeler., Reference 7 Above.

Oum, Tae., and Tretheway, Michael., "Airline Hub and Spoke Systems"., Transportation Research Forum., Vol. XXX, No. 2., 1990.

Gordon, S., and De Neufvill, R., "Design of Air Transportation Networks"., Transportation Research., Vol. 7., 1973.

See Higgins., and Toh., Reference 6 Above.

See Yaw Jeng., Reference 1 Above.

Richardson, Robert., "An Optimization Approach to Routing Aircraft"., Transportation Science., Vol. 10., P 52 - 71., 1976.

See Richardson., Reference 15 Above. 
Gordon, Steven., and De Neufville, Richard.,"Design Of Air Transportation Networks"., Transportation Research, Vol. 7, pp. 207-222., 1973.

Ferguson, A., and Dantzig, G. B., "The Problem Of Routing Aircraft, A Mathematical Solution"., Rand Report P-561, Rand Corporation., Santa Monica, California, Sept., 1954.

Etschmaier,M. M.,"Schedule Construction And Evaluation For Short And Medium Range Corporate Planning"., AGIFORS 10., 1970.

Kushige, T., "A Solution Of Most Profitable Aircraft Routing"., AGIFORS 3, 1963.

See Jeng., Reference 1 above.

Ghobrial, Atef., Balakrishnan, Nagraj., and Kanafani, Adib., "A Heuristic Model For Frequency Planning And Aircraft Routing In Small Size Airlines"., Transportation Planning And Technology., Vol. 16, PP. 235 - 249., 1992.

Bailey, E., Graham,D., and Kaplan, D.,"Deregulating The Airlines" MIT Press, Cambridge., MA., 1985.

Ghobrial,A.,"Analysis Of The Air Network Structure: The Hubbing Phenomenon"., Ph.D. Dissertation., University Of California., Berkeley., UCB-ITS-DS-83-2, PP. 138., 1983.

Chou,Y.H.,"The Hierarchical-Hub Model For Airline Networks"., Transportation Planning And Technology., Vol. 14, PP. 243 - 258., 1990.

Taaffe, E.J., and Gauthier, H. L., Geography of Transportation., Prentice-Hall., New Jersey., 1973.

Todoroki, T., Hanzawa, Y., and Fukuda, A., "Analysis Of Domestic Air Transportation Network In Indonesia"., The 6th World Conference On Transportation Research., Lyon France., 1992.

Nesa Wu., and Coppins, Richard., Linear Programming And Extensions., McGrawHill., 1981.

De Neufville, Richard., Applied Systems Analysis., McGraw-Hill., 1990.

See De Neuville., Reference 29 Above.

See De Neuville., Reference 29 Above.

Shaw, Stephen., Airline Marketing And Management., Pitman., 1985.

Gordon, S., and De Neufville, R., "Design Of Air Transportation Networks"., Transportation Research., Vol.7., 1973.

Oum, Tae H., and Tretheway, Michael W., "Airline Hub And Spoke Systems"., Journal Of The Transportation Research Forum., Vol. XXX., No. 2., 1990. 
Tarski, I., The Time Factor In Transportation Processes., Elsevier., Warsaw., Poland., 1987.

Bruzelius, Nils., The Value of travel Time., Croom Helm., London., 1979.

Beesley, M. E., " The Value of Time Spent Travelling: some New Evidence"., Economica., Vol. 32, PP. 174-85., 1965.

See Tarski., Reference 35 Above.

A. J. Harrison., and D. A. Quarmby., "The Value Of Time"., From Richard Layard (Editor)., Cost Benefit Analysis., Penguin., 1972.

A. J. Harrison., and D. A. Quarmby., "The Value Of Time"., From Richard Layard (Editor)., Cost Benefit Analysis., Penguin., 1972.

Gronau, R., "The value of Time In Passenger Transportation: The Demand For Air Travel"., National Bureau of Economic Research., NewYork., 1970.

Personal Interviews With Officials of Saudi Arabian Airlines., Jeddah., Saudi Arabia, August., 1992.

Personal Interviews With Saudi Arabian Airline Officials., Jeddah., Saudi Arabia., Aug., 1992.

Oum, Tae H., and Tretheway, Michael., "Airline Hub And Spoke Systems"., Journal Of The Transportation Research Forum., Vol. XXX., No. 2., 1990.

International Air Transport Association., World Air Transport Statistics., 1988.

International Air Transport Association., World Air Transport Statistics., 1988.

Kocks Consult GMBH., "Saudi Arabian National Transportation Plan"., Vol. 1 Executive Summary., Ministry Of Planning., Kingdom Of Saudi Arabia., 1982.

Saudia., "Timetable"., Saudi Arabian Airline., Jeddah., Saudi Arabia., July 1992.

Personal Interviews With Airline Officials, Jeddah., Saudi Arabia., August 1992.

Mark Hansen., And Adib Kanafani., "Hubbing And Airline Costs"., Journal of Transportation Engineering., Volume 115., No. 6., November., 1989.

See Gronau, R., Reference 41 Above. 
APPENDIX A 


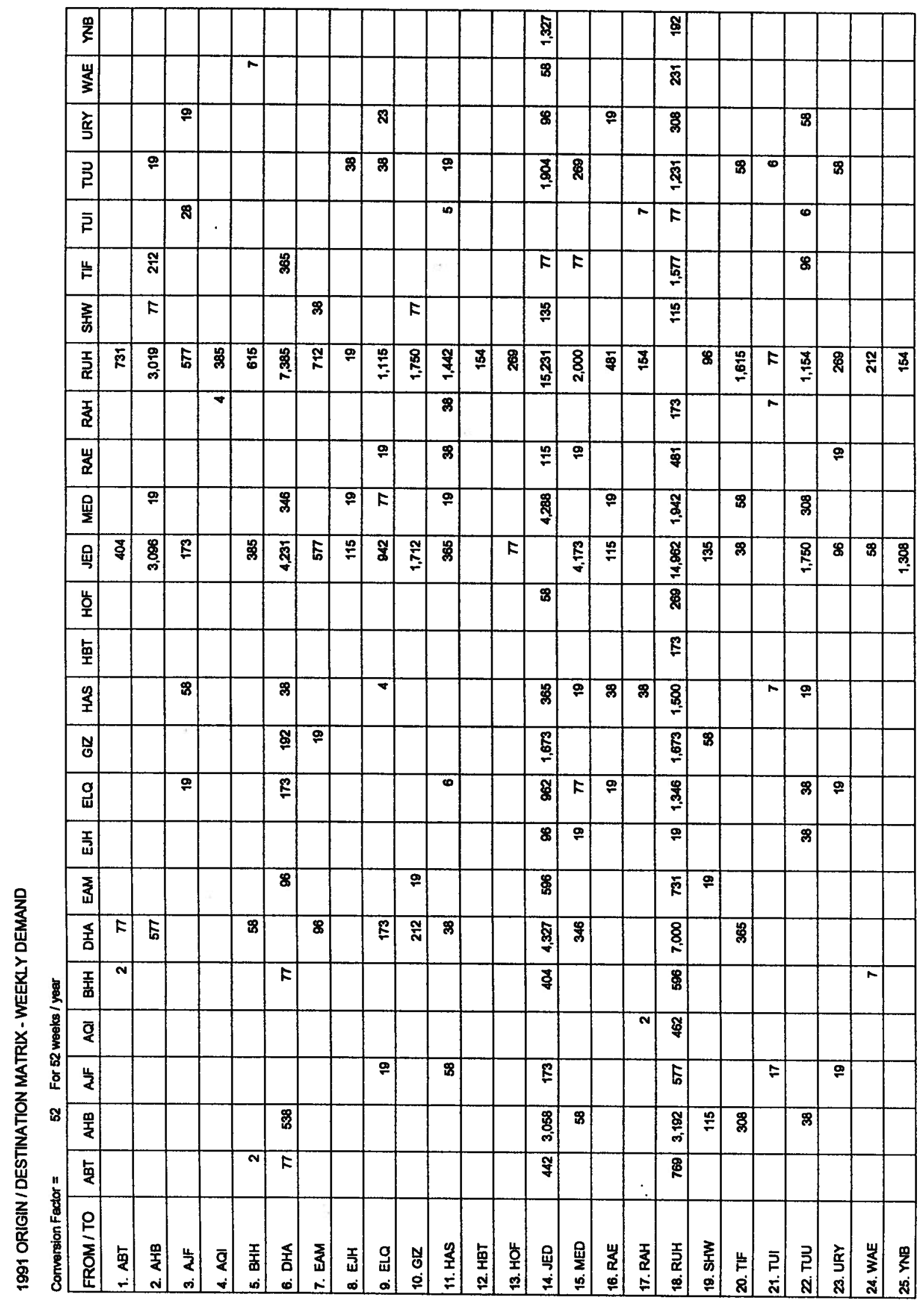


DATA FILE

Number of airports: 25

Value of Eime: 28.40

\begin{tabular}{|c|c|c|c|c|c|c|c|c|c|c|}
\hline From & To & Flow & Cost & Seats & Time & Dist & From & To & Flow & Cost \\
\hline 1 & 2 & 00 & $0.0 \overline{0}$ & 0 & $0.00^{-}$ & 0 & 2 & -1 & 0 & 0.00 \\
\hline 1 & 3 & 0 & 0.00 & 0 & 0.00 & 0 & 3 & 1 & 0 & 0.00 \\
\hline 1 & 4 & 0 & 0.00 & 0 & 0.00 & 0 & 4 & 1 & 0 & 0.00 \\
\hline 1 & 5 & 2 & 8.33 & 102 & 0.49 & 125 & 5 & 1 & 2 & 8.33 \\
\hline 1 & 6 & 77 & 66.97 & 102 & 1.81 & 607 & 6 & 1 & 77 & 66.97 \\
\hline 1 & 7 & 0 & 0.00 & 0 & 0.00 & 0 & 7 & 1 & 0 & 0.00 \\
\hline 1 & 8 & 0 & 0.00 & 0 & 0.00 & 0 & 8 & 1 & 0 & 0.00 \\
\hline 1 & 9 & 0 & 0.00 & 0 & 0.00 & 0 & 9 & 1 & 0 & 0.00 \\
\hline 1 & 10 & 0 & 0.00 & 0 & 0.00 & 0 & 10 & 1 & 0 & 0.00 \\
\hline 1 & 11 & 0 & 0.00 & 0 & 0.00 & 0 & 11 & 1 & 0 & 0.00 \\
\hline 1 & 12 & 0 & 0.00 & 0 & 0.00 & 0 & 12 & 1 & 0 & 0.00 \\
\hline 1 & 13 & 0 & 0.00 & 0 & 0.00 & 0 & 13 & 1 & 0 & 0.00 \\
\hline 1 & 14 & 404 & 37.13 & 258 & 0.79 & 106 & 14 & 1 & 442 & 37.24 \\
\hline 1 & 15 & 0 & 0.00 & 0 & 0.00 & 0 & 15 & 1 & 0 & 0.00 \\
\hline 1 & 16 & 0 & 0.00 & 0 & 0.00 & 0 & 16 & 1 & 0 & 0.00 \\
\hline 1 & 17 & 0 & 0.00 & 0 & 0.00 & 0 & 17 & 1 & 0 & 0.00 \\
\hline 1 & 18 & 731 & 43.23 & 201 & 1.31 & 362 & 18 & 1 & 769 & 52.37 \\
\hline 1 & 19 & 0 & 0.00 & 0 & 0.00 & 0 & 19 & 1 & 0 & 0.00 \\
\hline 1 & 20 & 0 & 0.00 & 0 & 0.00 & 0 & 20 & 1 & 0 & 0.00 \\
\hline 1 & 21 & 0 & 0.00 & 0 & 0.00 & 0 & 21 & 1 & 0 & 0.00 \\
\hline 1 & 22 & 0 & 0.00 & 0 & 0.00 & 0 & 22 & 1 & 0 & 0.00 \\
\hline 1 & 23 & 0 & 0.00 & 0 & 0.00 & 0 & 23 & 1 & 0 & 0.00 \\
\hline 1 & 24 & 0 & 0.00 & 0 & 0.00 & 0 & 24 & 1 & 0 & 0.00 \\
\hline 1 & 25 & 0 & 0.00 & 0 & 0.00 & 0 & 25 & 1 & 0 & 0.00 \\
\hline 2 & 3 & 0 & 0.00 & 0 & 0.00 & 0 & 3 & 2 & 0 & 0.00 \\
\hline 2 & 4 & 0 & 0.00 & 0 & 0.00 & 0 & 4 & 2 & 0 & 0.00 \\
\hline 2 & 5 & 0 & 0.00 & 0 & 0.00 & 0 & 5 & 2 & 0 & 0.00 \\
\hline 2 & 6 & 577 & 91.52 & 244 & 2.08 & 734 & 6 & 2 & 538 & 87.36 \\
\hline 2 & 7 & 0 & 0.00 & 0 & 0.00 & 0 & 7 & 2 & 0 & 0.00 \\
\hline 2 & 8 & 0 & 0.00 & 0 & 0.00 & 0 & 8 & 2 & 0 & 0.00 \\
\hline 2 & 9 & 0 & 0.00 & 0 & 0.00 & 0 & 9 & 2 & 0 & 0.00 \\
\hline 2 & 10 & 0 & 0.00 & 0 & 0.00 & 0 & 10 & 2 & 0 & 0.00 \\
\hline 2 & 11 & 0 & 0.00 & 0 & 0.00 & 0 & 11 & 2 & 0 & 0.00 \\
\hline 2 & 12 & 0 & 0.00 & 0 & 0.00 & 0 & 12 & 2 & 0 & 0.00 \\
\hline 2 & 13 & 0 & 0.00 & 0 & 0.00 & 0 & 13 & 2 & 0 & 0.00 \\
\hline 2 & 14 & 3096 & 45.67 & 202 & 1.10 & 286 & 14 & 2 & 3058 & 40.96 \\
\hline 2 & 15 & 19 & 54.36 & 102 & 1.51 & 466 & 15 & 2 & 58 & 54.36 \\
\hline 2 & 16 & 0 & 0.00 & 0 & 0.00 & 0 & 16 & 2 & 0 & 0.00 \\
\hline 2 & 17 & 0 & 0.00 & 0 & 0.00 & 0 & 17 & 2 & 0 & 0.00 \\
\hline 2 & 18 & 3019 & 58.74 & 202 & 1.54 & 461 & 18 & 2 & 3192 & 68.04 \\
\hline 2 & 19 & 77 & 15.68 & 102 & 0.99 & 298 & 19 & 2 & 115 & 17.68 \\
\hline 2 & 20 & 212 & 30.30 & 202 & 0.94 & 229 & 20 & 2 & 308 & 33.94 \\
\hline 2 & 21 & 0 & 0.00 & 0 & 0.00 & 0 & 21 & 2 & 0 & 0.00 \\
\hline 2 & 22 & 19 & 73.26 & 102 & 2.22 & 798 & 22 & 2 & 38 & 73.26 \\
\hline 2 & 23 & 0 & 0.00 & 0 & 0.00 & 0 & 23 & 2 & 0 & 0.00 \\
\hline 2 & 24 & 0 & 0.00 & 0 & 0.00 & 0 & 24 & 2 & 0 & 0.00 \\
\hline 2 & 25 & 0 & 0.00 & 0 & 0.00 & 0 & 25 & 2 & 0 & 0.00 \\
\hline 3 & 4 & 0 & 0.00 & 0 & 0.00 & 0 & 4 & 3 & 0 & 0.00 \\
\hline 3 & 5 & 0 & 0.00 & 0 & 0.00 & 0 & 5 & 3 & 0 & 0.00 \\
\hline 3 & 6 & 0 & 0.00 & 0 & 0.00 & 0 & 6 & 3 & 0 & 0.00 \\
\hline 3 & 7 & 0 & 0.00 & 0 & 0.00 & 0 & 7 & 3 & 0 & 0.00 \\
\hline 3 & 8 & 0 & 0.00 & 0 & 0.00 & 0 & 8 & 3 & 0 & 0.00 \\
\hline 3 & 9 & 19 & 24.00 & 102 & 1.08 & 333 & 9 & 3 & 19 & 20.13 \\
\hline
\end{tabular}


APPENDIX A:

DATA FILE

\begin{tabular}{|c|c|c|c|c|c|c|c|c|c|c|}
\hline 3 & 10 & 0 & 0.00 & 0 & 0.00 & 0 & 10 & 3 & 0 & 0.00 \\
\hline 3 & 11 & 58 & 18.75 & 102 & 0.80 & 184 & 11 & 3 & 58 & 15.12 \\
\hline 3 & 12 & 0 & 0.00 & 0 & 0.00 & 0 & 12 & 3 & 0 & 0.00 \\
\hline 3 & 13 & 0 & 0.00 & 0 & 0.00 & 0 & 13 & 3 & 0 & 0.00 \\
\hline 3 & 14 & 173 & 28.56 & 102 & 1.68 & 548 & 14 & 3 & 173 & 27.54 \\
\hline 3 & 15 & 0 & 0.00 & 0 & 0.00 & 0 & 15 & 3 & 0 & 0.00 \\
\hline 3 & 16 & 0 & 0.00 & 0 & 0.00 & 0 & 16 & 3 & 0 & 0.00 \\
\hline 3 & 17 & 0 & 0.00 & 0 & 0.00 & 0 & 17 & 3 & 0 & 0.00 \\
\hline 3 & 18 & 577 & 32.66 & 102 & 1.58 & 483 & 18 & 3 & 577 & 25.53 \\
\hline 3 & 19 & 0 & 0.00 & 0 & 0.00 & 0 & 19 & 3 & 0 & 0.00 \\
\hline 3 & 20 & 0 & 0.00 & 0 & 0.00 & 0 & 20 & 3 & 0 & 0.00 \\
\hline 3 & 21 & 28 & 9.38 & 102 & 0.67 & 149 & 21 & 3 & 17 & 14.74 \\
\hline 3 & 22 & 0 & 0.00 & 0 & 0.00 & 0 & 22 & 3 & 0 & 0.00 \\
\hline 3 & 23 & 19 & 18.04 & 102 & 0.78 & 158 & 23 & 3 & 19 & 11.25 \\
\hline 3 & 24 & 0 & 0.00 & 0 & 0.00 & 0 & 24 & 3 & 0 & 0.00 \\
\hline 3 & 25 & 0 & 0.00 & 0 & 0.00 & 0 & 25 & 3 & 0 & 0.00 \\
\hline 4 & 5 & 0 & 0.00 & 0 & 0.00 & 0 & 5 & 4 & 0 & 0.00 \\
\hline 4 & 6 & 0 & 0.00 & 0 & 0.00 & 0 & 6 & 4 & 0 & 0.00 \\
\hline 4 & 7 & 0 & 0.00 & 0 & 0.00 & 0 & 7 & 4 & 0 & 0.00 \\
\hline 4 & 8 & 0 & 0.00 & 0 & 0.00 & 0 & 8 & 4 & 0 & 0.00 \\
\hline 4 & 9 & 0 & 0.00 & 0 & 0.00 & 0 & 9 & 4 & 0 & 0.00 \\
\hline 4 & 10 & 0 & 0.00 & 0 & 0.00 & 0 & 10 & 4 & 0 & 0.00 \\
\hline 4 & 11 & 0 & 0.00 & 0 & 0.00 & 0 & 11 & 4 & 0 & 0.00 \\
\hline 4 & 12 & 0 & 0.00 & 0 & 0.00 & 0 & 12 & 4 & 0 & 0.00 \\
\hline 4 & 13 & 0 & 0.00 & 0 & 0.00 & 0 & 13 & 4 & 0 & 0.00 \\
\hline 4 & 14 & 0 & 0.00 & 0 & 0.00 & 0 & 14 & 4 & 0 & 0.00 \\
\hline 4 & 15 & 0 & 0.00 & 0 & 0.00 & 0 & 15 & 4 & 0 & 0.00 \\
\hline 4 & 16 & 0 & 0.00 & 0 & 0.00 & 0 & 16 & 4 & 0 & 0.00 \\
\hline 4 & 17 & 4 & 20.16 & 102 & 0.84 & 640 & 17 & 4 & 2 & 22.56 \\
\hline 4 & 18 & 385 & 22.22 & 102 & 0.92 & 219 & 18 & 4 & 462 & 16.73 \\
\hline 4 & 19 & 0 & 0.00 & 0 & 0.00 & 0 & 19 & 4 & 0 & 0.00 \\
\hline 4 & 20 & 0 & 0.00 & 0 & 0.00 & 0 & 20 & 4 & 0 & 0.00 \\
\hline 4 & 21 & 0 & 0.00 & 0 & 0.00 & 0 & 21 & 4 & 0 & 0.00 \\
\hline 4 & 22 & 0 & 0.00 & 0 & 0.00 & 0 & 22 & 4 & 0 & 0.00 \\
\hline 4 & 23 & 0 & 0.00 & 0 & 0.00 & 0 & 23 & 4 & 0 & 0.00 \\
\hline 4 & 24 & 0 & 0.00 & 0 & 0.00 & 0 & 24 & 4 & 0 & 0.00 \\
\hline 4 & 25 & 0 & 0.00 & 0 & 0.00 & 0 & 25 & 4 & 0 & 0.00 \\
\hline 5 & 6 & 58 & 32.64 & 102 & 1.92 & 661 & 6 & 5 & 77 & 28.80 \\
\hline 5 & 7 & 0 & 0.00 & 0 & 0.00 & 0 & 7 & 5 & 0 & 0.00 \\
\hline 5 & 8 & 0 & 0.00 & 0 & 0.00 & 0 & 8 & 5 & 0 & 0.00 \\
\hline 5 & 9 & 0 & 0.00 & 0 & 0.00 & 0 & 9 & 5 & 0 & 0.00 \\
\hline 5 & 10 & 0 & 0.00 & 0 & 0.00 & 0 & 10 & 5 & 0 & 0.00 \\
\hline 5 & 11 & 0 & 0.00 & 0 & 0.00 & 0 & 11 & 5 & 0 & 0.00 \\
\hline 5 & 12 & 0 & 0.00 & 0 & 0.00 & 0 & 12 & 5 & 0 & 0.00 \\
\hline 5 & 13 & 0 & 0.00 & 0 & 0.00 & 0 & 13 & 5 & 0 & 0.00 \\
\hline 5 & 14 & 385 & 17.58 & 102 & 0.99 & 227 & 14 & 5 & 404 & 18.03 \\
\hline 5 & 15 & 0 & 0.00 & 0 & 0.00 & 0 & 15 & 5 & 0 & 0.00 \\
\hline 5 & 16 & 0 & 0.00 & 0 & 0.00 & 0 & 16 & 5 & 0 & 0.00 \\
\hline 5 & 17 & 0 & 0.00 & 0 & 0.00 & 0 & 17 & 5 & 0 & 0.00 \\
\hline 5 & 18 & 615 & 20.44 & 102 & 1.34 & 361 & 18 & 5 & 596 & 21.32 \\
\hline 5 & 19 & 0 & 0.00 & 0 & 0.00 & 0 & 19 & 5 & 0 & 0.00 \\
\hline 5 & 20 & 0 & 0.00 & 0 & 0.00 & 0 & 20 & 5 & 0 & 0.00 \\
\hline 5 & 21 & 0 & 0.00 & 0 & 0.00 & 0 & 21 & 5 & 0 & 0.00 \\
\hline 5 & 22 & 0 & 0.00 & 0 & 0.00 & 0 & 22 & 5 & 0 & 0.00 \\
\hline 5 & 23 & 0 & 0.00 & 0 & 0.00 & 0 & 23 & 5 & 0 & 0.00 \\
\hline 5 & 24 & 7 & 10.98 & 102 & 0.61 & 149 & 24 & 5 & 7 & 10.72 \\
\hline 5 & 25 & 0 & 0.00 & 0 & 0.00 & 0 & 25 & 5 & 0 & 0.00 \\
\hline 6 & 7 & 96 & 28.28 & 102 & 2.02 & 704 & 7 & 6 & 96 & 38.38 \\
\hline 6 & 8 & 0 & 0.00 & 0 & 0.00 & 0 & 8 & 6 & 0 & 0.00 \\
\hline 6 & 9 & 173 & 21.75 & 102 & 1.25 & 391 & 9 & 6 & 173 & 30.45 \\
\hline
\end{tabular}


APPENDIX A:

DATA FILE

\begin{tabular}{|c|c|c|c|c|c|c|c|c|c|c|}
\hline 6 & 10 & 192 & 32.62 & 102 & 2.14 & 808 & 10 & 6 & 212 & 30.07 \\
\hline 6 & 11 & 38 & 24.90 & 102 & 1.66 & 538 & 11 & 6 & 38 & 29.88 \\
\hline 6 & 12 & 0 & 0.00 & 0 & 0.00 & 0 & 12 & 6 & 0 & 0.00 \\
\hline 6 & 13 & 0 & 0.00 & 0 & 0.00 & 0 & 13 & 6 & 0 & 0.00 \\
\hline 6 & 14 & 4231 & 96.80 & 253 & 2.01 & 763 & 14 & 6 & 4327 & 86.56 \\
\hline 6 & 15 & 346 & 55.92 & 180 & 1.82 & 620 & 15 & 6 & 346 & 41.58 \\
\hline 6 & 16 & 0 & 0.00 & 0 & 0.00 & 0 & 16 & 6 & 0 & 0.00 \\
\hline 6 & 17 & 0 & 0.00 & 0 & 0.00 & 0 & 17 & 6 & 0 & 0.00 \\
\hline 6 & 18 & 7385 & 38.76 & 251 & 0.99 & 257 & 18 & 6 & 7000 & 39.11 \\
\hline 6 & 19 & 0 & 0.00 & 0 & 0.00 & 0 & 19 & 6 & 0 & 0.00 \\
\hline 6 & 20 & 365 & 89.30 & 102 & 1.90 & 650 & 20 & 6 & 365 & 89.30 \\
\hline 6 & 21 & 0 & 0.00 & 0 & 0.00 & 0 & 21 & 6 & 0 & 0.00 \\
\hline 6 & 22 & 0 & 0.00 & 0 & 0.00 & 0 & 22 & 6 & 0 & 0.00 \\
\hline 6 & 23 & 0 & 0.00 & 0 & 0.00 & 0 & 23 & 6 & 0 & 0.00 \\
\hline 6 & 24 & 0 & 0.00 & 0 & 0.00 & 0 & 24 & 6 & 0 & 0.00 \\
\hline 6 & 25 & 0 & 0.00 & 0 & 0.00 & 0 & 25 & 6 & 0 & 0.00 \\
\hline 7 & 8 & 0 & 0.00 & 0 & 0.00 & 0 & 8 & 7 & 0 & 0.00 \\
\hline 7 & 9 & 0 & 0.00 & 0 & 0.00 & 0 & 9 & 7 & 0 & 0.00 \\
\hline 7 & 10 & 19 & 12.75 & 102 & 0.75 & 159 & 10 & 7 & 19 & 12.75 \\
\hline 7 & 11 & 0 & 0.00 & 0 & 0.00 & 0 & 11 & 7 & 0 & 0.00 \\
\hline 7 & 12 & 0 & 0.00 & 0 & 0.00 & 0 & 12 & 7 & 0 & 0.00 \\
\hline 7 & 13 & 0 & 0.00 & 0 & 0.00 & 0 & 13 & 7 & 0 & 0.00 \\
\hline 7 & 14 & 577 & 24.12 & 102 & 1.33 & 389 & 14 & 7 & 596 & 21.56 \\
\hline 7 & 15 & 0 & 0.00 & 0 & 0.00 & 0 & 15 & 7 & 0 & 0.00 \\
\hline 7 & 16 & 0 & 0.00 & 0 & 0.00 & 0 & 16 & 7 & 0 & 0.00 \\
\hline 7 & 17 & 0 & 0.00 & 0 & 0.00 & 0 & 17 & 7 & 0 & 0.00 \\
\hline 7 & 18 & 712 & 23.97 & 102 & 1.53 & 514 & 18 & 7 & 731 & 24.75 \\
\hline 7 & 19 & 38 & 12.24 & 102 & 0.72 & 158 & 19 & 7 & 19 & 12.75 \\
\hline 7 & 20 & 0 & 0.00 & 0 & 0.00 & 0 & 20 & 7 & 0 & 0.00 \\
\hline 7 & 21 & 0 & 0.00 & 0 & 0.00 & 0 & 21 & 7 & 0 & 0.00 \\
\hline 7 & 22 & 0 & 0.00 & 0 & 0.00 & 0 & 22 & 7 & 0 & 0.00 \\
\hline 7 & 23 & 0 & 0.00 & 0 & 0.00 & 0 & 23 & 7 & 0 & 0.00 \\
\hline 7 & 24 & 0 & 0.00 & 0 & 0.00 & 0 & 24 & 7 & 0 & 0.00 \\
\hline 7 & 25 & 0 & 0.00 & 0 & 0.00 & 0 & 25 & 7 & 0 & 0.00 \\
\hline 8 & 9 & 0 & 0.00 & 0 & 0.00 & 0 & 9 & 8 & 0 & 0.00 \\
\hline 8 & 10 & 0 & 0.00 & 0 & 0.00 & 0 & 10 & 8 & 0 & 0.00 \\
\hline 8 & 11 & 0 & 0.00 & 0 & 0.00 & 0 & 11 & 8 & 0 & 0.00 \\
\hline 8 & 12 & 0 & 0.00 & 0 & 0.00 & 0 & 12 & 8 & 0 & 0.00 \\
\hline 8 & 13 & 0 & 0.00 & 0 & 0.00 & 0 & 13 & 8 & 0 & 0.00 \\
\hline 8 & 14 & 115 & 24.50 & 102 & 1.08 & 324 & 14 & 8 & 96 & 24.64 \\
\hline 8 & 15 & 19 & 21.16 & 102 & 0.89 & 235 & 15 & 8 & 19 & 18.27 \\
\hline 8 & 16 & 0 & 0.00 & 0 & 0.00 & 0 & 16 & 8 & 0 & 0.00 \\
\hline 8 & 17 & 0 & 0.00 & 0 & 0.00 & 0 & 17 & 8 & 0 & 0.00 \\
\hline 8 & 18 & 19 & 35.91 & 102 & 1.89 & 646 & 18 & 8 & 19 & 28.35 \\
\hline 8 & 19 & 0 & 0.00 & 0 & 0.00 & 0 & 19 & 8 & 0 & 0.00 \\
\hline 8 & 20 & 0 & 0.00 & 0 & 0.00 & 0 & 20 & 8 & 0 & 0.00 \\
\hline 8 & 21 & 0 & 0.00 & 0 & 0.00 & 0 & 21 & 8 & 0 & 0.00 \\
\hline 8 & 22 & 38 & 16.03 & 102 & 0.66 & 175 & 22 & 8 & 38 & 15.63 \\
\hline 8 & 23 & 0 & 0.00 & 0 & 0.00 & 0 & 23 & 8 & 0 & 0.00 \\
\hline 8 & 24 & 0 & 0.00 & 0 & 0.00 & 0 & 24 & 8 & 0 & 0.00 \\
\hline 8 & 25 & 0 & 0.00 & 0 & 0.00 & 0 & 25 & 8 & 0 & 0.00 \\
\hline 9 & 10 & 0 & 0.00 & 0 & 0.00 & 0 & 10 & 9 & 0 & 0.00 \\
\hline 9 & 11 & 4 & 12.78 & 102 & 0.71 & 175 & 11 & 9 & 6 & 12.75 \\
\hline 9 & 12 & 0 & 0.00 & 0 & 0.00 & 0 & 12 & 9 & 0 & 0.00 \\
\hline 9 & 13 & 0 & 0.00 & 0 & 0.00 & 0 & 13 & 9 & 0 & 0.00 \\
\hline 9 & 14 & 942 & 51.12 & 201 & 1.34 & 443 & 14 & 9 & 962 & 42.16 \\
\hline 9 & 15 & 77 & 49.95 & 251 & 1.12 & 284 & 15 & 9 & 77 & 51.52 \\
\hline 9 & 16 & 19 & 19.80 & 102 & 1.12 & 365 & 16 & 9 & 19 & 19.32 \\
\hline 9 & 17 & 0 & 0.00 & 0 & 0.00 & 0 & 17 & 9 & 0 & 0.0 \\
\hline 9 & 18 & 1115 & 21.59 & 201 & 0.86 & 210 & 18 & 9 & 1348 & 23.5 \\
\hline
\end{tabular}


APPENDIX A:

DATA FILE

\begin{tabular}{|c|c|c|c|c|c|c|c|c|c|c|}
\hline 9 & 19 & 0 & 0.00 & 0 & 0.00 & 0 & 19 & 9 & 0 & 0.00 \\
\hline 9 & 20 & 0 & 0.00 & 0 & 0.00 & 0 & 20 & 9 & 0 & 0.00 \\
\hline 9 & 21 & 0 & 0.00 & 0 & 0.00 & 0 & 21 & 9 & 0 & 0.00 \\
\hline 9 & 22 & 38 & 22.88 & 102 & 1.29 & 466 & 22 & 9 & 38 & 19.55 \\
\hline 9 & 23 & 23 & 22.35 & 102 & 1.49 & 529 & 23 & 9 & 19 & 19.20 \\
\hline 9 & 24 & 0 & 0.00 & 0 & 0.00 & 0 & 24 & 9 & 0 & 0.00 \\
\hline 9 & 25 & 0 & 0.00 & 0 & 0.00 & 0 & 25 & 9 & 0 & 0.00 \\
\hline 10 & 11 & 0 & 0.00 & 0 & 0.00 & 0 & 11 & 10 & 0 & 0.00 \\
\hline 10 & 12 & 0 & 0.00 & 0 & 0.00 & 0 & 12 & 10 & 0 & 0.00 \\
\hline 10 & 13 & 0 & 0.00 & 0 & 0.00 & 0 & 13 & 10 & 0 & 0.00 \\
\hline 10 & 14 & 1712 & 22.80 & 102 & 1.19 & 336 & 14 & 10 & 1673 & 20.32 \\
\hline 10 & 15 & 0 & 0.00 & 0 & 0.00 & 0 & 15 & 10 & 0 & 0.00 \\
\hline 10 & 16 & 0 & 0.00 & 0 & 0.00 & 0 & 16 & 10 & 0 & 0.00 \\
\hline 10 & 17 & 0 & 0.00 & 0 & 0.00 & 0 & 17 & 10 & 0 & 0.00 \\
\hline 10 & 18 & 1750 & 25.21 & 102 & 1.45 & 565 & 18 & 10 & 1673 & 19.01 \\
\hline 10 & 19 & 77 & 17.28 & 102 & 0.89 & 150 & 19 & 10 & 58 & 12.87 \\
\hline 10 & 20 & 0 & 0.00 & 0 & 0.00 & 0 & 20 & 10 & 0 & 0.00 \\
\hline 10 & 21 & 0 & 0.00 & 0 & 0.00 & 0 & 21 & 10 & 0 & 0.00 \\
\hline 10 & 22 & 0 & 0.00 & 0 & 0.00 & 0 & 22 & 10 & 0 & 0.00 \\
\hline 10 & 23 & 0 & 0.00 & 0 & 0.00 & 0 & 23 & 10 & 0 & 0.00 \\
\hline 10 & 24 & 0 & 0.00 & 0 & 0.00 & 0 & 24 & 10 & 0 & 0.00 \\
\hline 10 & 25 & 0 & 0.00 & 0 & 0.00 & 0 & 25 & 10 & 0 & 0.00 \\
\hline 11 & 12 & 0 & 0.00 & 0 & 0.00 & 0 & 12 & 11 & 0 & 0.00 \\
\hline 11 & 13 & 0 & 0.00 & 0 & 0.00 & 0 & 13 & 11 & 0 & 0.00 \\
\hline 11 & 14 & 365 & 34.95 & 180 & 1.28 & 393 & 14 & 11 & 365 & 27.24 \\
\hline 11 & 15 & 19 & 29.82 & 180 & 0.85 & 231 & 15 & 11 & 19 & 22.64 \\
\hline 11 & 16 & 38 & 16.82 & 102 & 1.21 & 245 & 16 & 11 & 38 & 31.97 \\
\hline 11 & 17 & 38 & 12.92 & 102 & 0.73 & 192 & 17 & 11 & 38 & 19.00 \\
\hline 11 & 18 & 1442 & 33.65 & 180 & 1.16 & 386 & 18 & 11 & 1500 & 33.34 \\
\hline 11 & 19 & 0 & 0.00 & 0 & 0.00 & 0 & 19 & 11 & 0 & 0.00 \\
\hline 11 & 20 & 0 & 0.00 & 0 & 0.00 & 0 & 20 & 11 & 0 & 0.00 \\
\hline 11 & 21 & 5 & 16.95 & 102 & 1.13 & 291 & 21 & 11 & 7 & 21.34 \\
\hline 11 & 22 & 19 & 49.45 & 258 & 1.08 & 306 & 22 & 11 & 19 & 44.95 \\
\hline 11 & 23 & 0 & 0.00 & 0 & 0.00 & 0 & 23 & 11 & 0 & 0.00 \\
\hline 11 & 24 & 0 & 0.00 & 0 & 0.00 & 0 & 24 & 11 & 0 & 0.00 \\
\hline 11 & 25 & 0 & 0.00 & 0 & 0.00 & 0 & 25 & 11 & 0 & 0.00 \\
\hline 12 & 13 & 0 & 0.00 & 0 & 0.00 & 0 & 13 & 12 & 0 & 0.00 \\
\hline 12 & 14 & 0 & 0.00 & 0 & 0.00 & 0 & 14 & 12 & 0 & 0.00 \\
\hline 12 & 15 & 0 & 0.00 & 0 & 0.00 & 0 & 15 & 12 & 0 & 0.00 \\
\hline 12 & 16 & 0 & 0.00 & 0 & 0.00 & 0 & 16 & 12 & 0 & 0.00 \\
\hline 12 & 17 & 0 & 0.00 & 0 & 0.00 & 0 & 17 & 12 & 0 & 0.00 \\
\hline 12 & 18 & 154 & 13.92 & 102 & 0.91 & 245 & 18 & 12 & 173 & 15.04 \\
\hline 12 & 19 & 0 & 0.00 & 0 & 0.00 & 0 & 19 & 12 & 0 & 0.00 \\
\hline 12 & 20 & 0 & 0.00 & 0 & 0.00 & 0 & 20 & 12 & 0 & 0.00 \\
\hline 12 & 21 & 0 & 0.00 & 0 & 0.00 & 0 & 21 & 12 & 0 & 0.00 \\
\hline 12 & 22 & 0 & 0.00 & 0 & 0.00 & 0 & 22 & 12 & 0 & 0.00 \\
\hline 12 & 23 & 0 & 0.00 & 0 & 0.00 & 0 & 23 & 12 & 0 & 0.00 \\
\hline 12 & 24 & 0 & 0.00 & 0 & 0.00 & 0 & 24 & 12 & 0 & 0.00 \\
\hline 12 & 25 & 0 & 0.00 & 0 & 0.00 & 0 & 25 & 12 & 0 & 0.00 \\
\hline 13 & 14 & 77 & 32.64 & 102 & 2.04 & 717 & 14 & 13 & 58 & 32.64 \\
\hline 13 & 15 & 0 & 0.00 & 0 & 0.00 & 0 & 15 & 13 & 0 & 0.00 \\
\hline 13 & 16 & 0 & 0.00 & 0 & 0.00 & 0 & 16 & 13 & 0 & 0.00 \\
\hline 13 & 17 & 0 & 0.00 & 0 & 0.00 & 0 & 17 & 13 & 0 & 0.00 \\
\hline 13 & 18 & 269 & 15.93 & 102 & 0.82 & 191 & 18 & 13 & 269 & 16.04 \\
\hline 13 & 19 & 0 & 0.00 & 0 & 0.00 & 0 & 19 & 13 & 0 & 0.00 \\
\hline 13 & 20 & 0 & 0.00 & 0 & 0.00 & 0 & 20 & 13 & 0 & 0.00 \\
\hline 13 & 21 & 0 & 0.00 & 0 & 0.00 & 0 & 21 & 13 & 0 & 0.00 \\
\hline 13 & 22 & 0 & 0.00 & 0 & 0.00 & 0 & 22 & 13 & 0 & 0.00 \\
\hline 13 & 23 & 0 & 0.00 & 0 & 0.00 & 0 & 23 & 13 & 0 & 0.00 \\
\hline 13 & 24 & 0 & 0.00 & 0 & 0.00 & 0 & 24 & 13 & 0 & 0.00 \\
\hline
\end{tabular}


APPENDIX A:

DATA FILE

\begin{tabular}{|c|c|c|c|c|c|c|c|c|c|c|}
\hline 13 & 25 & 0 & 0.00 & 0 & 0.00 & 0 & 25 & 13 & 0 & 0.00 \\
\hline 14 & 15 & 4288 & 40.45 & 253 & 0.86 & 213 & 15 & 14 & 4173 & 41.12 \\
\hline 14 & 16 & 115 & 35.68 & 102 & 2.23 & 805 & 16 & 14 & 115 & 42.37 \\
\hline 14 & 17 & 0 & 0.00 & 0 & 0.00 & 0 & 17 & 14 & 0 & 0.00 \\
\hline 14 & 18 & 15231 & 73.37 & 253 & 1.59 & 522 & 18 & 14 & 14962 & 76.78 \\
\hline 14 & 19 & 135 & 29.92 & 102 & 1.76 & 586 & 19 & 14 & 135 & 29.92 \\
\hline 14 & 20 & 77 & 26.39 & 201 & 0.64 & 95 & 20 & 14 & 38 & 29.50 \\
\hline 14 & 21 & 0 & 0.00 & 0 & 0.00 & 0 & 21 & 14 & 0 & 0.00 \\
\hline 14 & 22 & 1904 & 53.07 & 249 & 1.52 & 503 & 22 & 14 & 1750 & 52.64 \\
\hline 14 & 23 & 96 & 35.82 & 102 & 1.99 & 692 & 23 & 14 & 96 & 35.82 \\
\hline 14 & 24 & 58 & 25.08 & 102 & 1.32 & 377 & 24 & 14 & 58 & 22.44 \\
\hline 14 & 25 & 1327 & 13.86 & 102 & 0.77 & 197 & 25 & 14 & 1308 & 14.88 \\
\hline 15 & 16 & 19 & 19.04 & 102 & 1.27 & 448 & 16 & 15 & 19 & 25.96 \\
\hline 15 & 17 & 0 & 0.00 & 0 & 0.00 & 0 & 17 & 15 & 0 & 0.00 \\
\hline 15 & 18 & 2000 & 41.61 & 201 & 1.45 & 440 & 18 & 15 & 1942 & 53.71 \\
\hline 15 & 19 & 0 & 0.00 & 0 & 0.00 & 0 & 19 & 15 & 0 & 0.00 \\
\hline 15 & 20 & 77 & 48.02 & 102 & 0.92 & 218 & 20 & 15 & 58 & 31.24 \\
\hline 15 & 21 & 0 & 0.00 & 0 & 0.00 & 0 & 21 & 15 & 0 & 0.00 \\
\hline 15 & 22 & 269 & 36.02 & 201 & 1.03 & 315 & 22 & 15 & 308 & 34.79 \\
\hline 15 & 23 & 0 & 0.00 & 0 & 0.00 & 0 & 23 & 15 & 0 & 0.00 \\
\hline 15 & 24 & 0 & 0.00 & 0 & 0.00 & 0 & 24 & 15 & 0 & 0.00 \\
\hline 15 & 25 & 0 & 0.00 & 0 & 0.00 & 0 & 25 & 15 & 0 & 0.00 \\
\hline 16 & 17 & 0 & 0.00 & 0 & 0.00 & 0 & 17 & 16 & 0 & 0.00 \\
\hline 16 & 18 & 481 & 29.90 & 102 & 1.61 & 547 & 18 & 16 & 481 & 24.53 \\
\hline 16 & 19 & 0 & 0.00 & 0 & 0.00 & 0 & 19 & 16 & 0 & 0.00 \\
\hline 16 & 20 & 0 & 0.00 & 0 & 0.00 & 0 & 20 & 16 & 0 & 0.00 \\
\hline 16 & 21 & 0 & 0.00 & 0 & 0.00 & 0 & 21 & 16 & 0 & 0.00 \\
\hline 16 & 22 & 0 & 0.00 & 0 & 0.00 & 0 & 22 & 16 & 0 & 0.00 \\
\hline 16 & 23 & 19 & 18.66 & 102 & 0.86 & 230 & 23 & 16 & 19 & 10.68 \\
\hline 16 & 24 & 0 & 0.00 & 0 & 0.00 & 0 & 24 & 16 & 0 & 0.00 \\
\hline 16 & 25 & 0 & 0.00 & 0 & 0.00 & 0 & 25 & 16 & 0 & 0.00 \\
\hline 17 & 18 & 154 & 30.59 & 102 & 1.41 & 393 & 18 & 17 & 173 & 20.45 \\
\hline 17 & 19 & 0 & 0.00 & 0 & 0.00 & 0 & 19 & 17 & 0 & 0.00 \\
\hline 17 & 20 & 0 & 0.00 & 0 & 0.00 & 0 & 20 & 17 & 0 & 0.00 \\
\hline 17 & 21 & 7 & 25.83 & 102 & 1.23 & 321 & 21 & 17 & 7 & 23.31 \\
\hline 17 & 22 & 0 & 0.00 & 0 & 0.00 & 0 & 22 & 17 & 0 & 0.00 \\
\hline 17 & 23 & 0 & 0.00 & 0 & 0.00 & 0 & 23 & 17 & 0 & 0.00 \\
\hline 17 & 24 & 0 & 0.00 & 0 & 0.00 & 0 & 24 & 17 & 0 & 0.00 \\
\hline 17 & 25 & 0 & 0.00 & 0 & 0.00 & 0 & 25 & 17 & 0 & 0.00 \\
\hline 18 & 19 & 115 & 19.50 & 102 & 1.50 & 462 & 19 & 18 & 96 & 24.00 \\
\hline 18 & 20 & 1577 & 56.21 & 201 & 1.46 & 451 & 20 & 18 & 1615 & 53.66 \\
\hline 18 & 21 & 77 & 32.16 & 102 & 2.01 & 701 & 21 & 18 & 77 & 42.21 \\
\hline 18 & 22 & 1231 & 55.96 & 201 & 1.75 & 671 & 22 & 18 & 1154 & 47.85 \\
\hline 18 & 23 & 308 & 29.03 & 102 & 1.90 & 736 & 23 & 18 & 269 & 22.28 \\
\hline 18 & 24 & 231 & 18.30 & 102 & 1.13 & 293 & 24 & 18 & 212 & 17.68 \\
\hline 18 & 25 & 192 & 25.48 & 102 & 1.60 & 545 & 25 & 18 & 154 & 22.08 \\
\hline 19 & 20 & 0 & 0.00 & 0 & 0.00 & 0 & 20 & 19 & 0 & 0.00 \\
\hline 19 & 21 & 0 & 0.00 & 0 & 0.00 & 0 & 21 & 19 & 0 & 0.00 \\
\hline 19 & 22 & 0 & 0.00 & 0 & 0.00 & 0 & 22 & 19 & 0 & 0.00 \\
\hline 19 & 23 & 0 & 0.00 & 0 & 0.00 & 0 & 23 & 19 & 0 & 0.00 \\
\hline 19 & 24 & 0 & 0.00 & 0 & 0.00 & 0 & 24 & 19 & 0 & 0.00 \\
\hline 19 & 25 & 0 & 0.00 & 0 & 0.00 & 0 & 25 & 19 & 0 & 0.00 \\
\hline 20 & 21 & 0 & 0.00 & 0 & 0.00 & 0 & 21 & 20 & 0 & 0.00 \\
\hline 20 & 22 & 58 & 61.05 & 258 & 1.65 & 535 & 22 & 20 & 96 & 61.05 \\
\hline 20 & 23 & 0 & 0.00 & 0 & 0.00 & 0 & 23 & 20 & 0 & 0.00 \\
\hline 20 & 24 & 0 & 0.00 & 0 & 0.00 & 0 & 24 & 20 & 0 & 0.00 \\
\hline 20 & 25 & 0 & 0.00 & 0 & 0.00 & 0 & 25 & 20 & 0 & 0.00 \\
\hline 21 & 22 & 6 & 20.79 & 102 & 0.99 & 317 & 22 & 21 & 6 & 13.16 \\
\hline 21 & 23 & 0 & 0.00 & 0 & 0.00 & 0 & 23 & 21 & 0 & 0.00 \\
\hline 21 & 24 & 0 & 0.00 & 0 & 0.00 & 0 & 24 & 21 & 0 & 0.00 \\
\hline
\end{tabular}


APPENDIX A:

DATA FILE

$\begin{array}{rrrrrrlllrr}21 & 25 & 0 & 0.00 & 0 & 0.00 & 0 & 25 & 21 & 0 & 0.00 \\ 22 & 23 & 58 & 14.25 & 102 & 0.95 & 198 & 23 & 22 & 58 & 13.30 \\ 22 & 24 & 0 & 0.00 & 0 & 0.00 & 0 & 24 & 22 & 0 & 0.00 \\ 22 & 25 & 0 & 0.00 & 0 & 0.00 & 0 & 25 & 22 & 0 & 0.00 \\ 23 & 24 & 0 & 0.00 & 0 & 0.00 & 0 & 24 & 23 & 0 & 0.00 \\ 23 & 25 & 0 & 0.00 & 0 & 0.00 & 0 & 25 & 23 & 0 & 0.00 \\ 24 & 25 & 0 & 0.00 & 0 & 0.00 & 0 & 25 & 24 & 0 & 0.00\end{array}$


APPENDIX B 


\section{APPENDIX B : PROBLEM FORMULATION FILE EFFICIENT AIRLINE NETWORK}

MIN

+4165.00Y1T5+1212.28Y1T6T5+360.54Y1T14T5+ 449.82Y1T18T5+869.74Y1T6+589.00Y1T5T6+ 508.17Y1T14T6+427.78Y1T18T6+183.81Y1T14+ 260.32Y1T5T14+1092.96Y1T6T14+533.33Y1T18T14+ 236.55Y1T18+303.51Y1T5T18+820.67Y1T6T18+ 457.75Y1T14T18+475.84Y2T6+589.26Y2T14T6+ 817.54Y2T15T6+454.04Y2T18T6+1101.60Y2T20T6+ 236.02Y2T14+781.80Y2T6T14+705.03Y2T15T14+ 603.92Y2T18T14+454.46Y2T19T14+306.32Y2T20 T14+ 946.32Y2T22T14+2861.05Y2T15+920.29Y2T6T15+ 403.63Y2T14T15+583.25Y2T18T15+668.05Y2T20T15+ $2140.68 \mathrm{Y} 2 \mathrm{~T} 22 \mathrm{~T} 15+291.85 \mathrm{Y} 2 \mathrm{~T} 18+538.23 \mathrm{Y} 2 \mathrm{~T} 6 \mathrm{~T} 18+$ 525.00Y2T14T18+744.06Y2T15T18+395.85Y2T19T18+ 427.96Y2T20T18+964.23Y2T22T18+203.64Y2T19+ 653.69Y2T14T19+506.69Y2T18T19+285.85Y2T20+ 1392.58Y2T6T20+417.33Y2T14T20+1204.45Y2T15T20+ 573.66Y2T18T20+1347.86Y2T22T20+3855.79Y2T22+ 455.36Y2T14T22+1680.67Y2T15T22+622.74Y2T18T22+ 1055.19Y2T20T22+1263.16Y3T9+753.51Y3T11T9+ 512.38Y3T14T9+449.54Y3T18T9+980.00Y3T23T9+ 323.28Y3T11+517.82Y3T9T11+564.10Y3T14T11+ 552.62Y3T18T11+437.38Y3T21T11+330.17Y3T14+ 525.09Y3T9T14+438.39Y3T11T14+652.75Y3T18T14+ 587.40Y3T23T14+339.62Y3T18+356.45Y3T9T18+ 406.69Y3T11T18+597.04Y3T14T18+544.81Y3T21T18+ 418.63Y3T23T18+335.00Y3T21+731.66Y3T11T21+ 936.47Y3T18T21+949.47Y3T23+1163.72Y3T9T23+ $920.46 \mathrm{Y} 3 \mathrm{~T} 14 \mathrm{~T} 23+683.90 \mathrm{Y} 3 \mathrm{~T} 18 \mathrm{~T} 23+5040.00 \mathrm{Y} 4 \mathrm{~T} 17+$ $459.56 \mathrm{Y} 4 \mathrm{~T} 18 \mathrm{~T} 17+230.86 \mathrm{YT} 18+547.82 \mathrm{Y} 4 \mathrm{~T} 17 \mathrm{~T} 18+$ 4165.00Y5T1+1391.72Y5T6T1+349.45Y5T14T1+ $503.60 \mathrm{Y} 5 \mathrm{~T} 18 \mathrm{~T} 1+562.76 \mathrm{Y} 5 \mathrm{~T} 6+1130.98 \mathrm{YTT} 1 \mathrm{~T} 6+$ 553.74Y5T14T6+373.30Y5T18T6+182.65Y5T14+ 274.34Y5T1T14+766.84Y5T6T14+509.58Y5T18T14+ 365.31Y5T24T14+232.65Y5T18+319.33Y5T1T18+ 494.53Y5T6T18+467.50Y5T14T18+315.98Y5T24T18+ 1568.57Y5T24+565.23Y5T14T24+460.70Y5T18T24+ 869.74Y6T1+479.47Y6T5T1+619.72Y6T14T1+ 465.34Y6T18T1+487.14Y6T2+590.69Y6T14T2+ 863.54Y6T15T2+503.13Y6T18T2+1090.62Y6T20T2+ 374.03Y6T5+975.18Y6T1T5+591.88Y6T14T5+ 377.58Y6T18T5+294.58Y6T7+561.53Y6T10T7+ 620.77Y6T14T7+424.78Y6T18T7+251.45Y6T9+ 496.49Y6T11 T9+618.51Y6T14T9+529.32Y6T15T9+ 283.00Y6T18T9+339.79Y6T10+475.86Y6T7T10+ $600.95 \mathrm{Y} 6 \mathrm{~T} 14 \mathrm{~T} 10+352.25 \mathrm{Y} 6 \mathrm{~T} 18 \mathrm{~T} 10+655.26 \mathrm{Y} 6 \mathrm{~T} 11+$ $613.53 \mathrm{Y} 6 \mathrm{~T} 9 \mathrm{~T} 11+588.26 \mathrm{Y} 6 \mathrm{~T} 14 \mathrm{~T} 11+834.07 \mathrm{Y} 6 \mathrm{~T} 15 \mathrm{~T} 11+$ $351.75 \mathrm{Y} 6 \mathrm{~T} 18 \mathrm{~T} 11+388.94 \mathrm{Y} 6 \mathrm{~T} 14+812.65 \mathrm{Y} 6 \mathrm{~T} 1 \mathrm{~T} 14+$ 596.99Y6T2T14+462.66Y6T5T14+521.83Y6T7T14+ 474.24Y6T9T14+550.85Y6T10T14+442.69Y6T11T14+ 484.02Y6T15T14+460.86Y6T18T14+1045.80Y6T20 T14+ 323.24Y6T15+991.02Y6T2T15+487.62Y6T9T15+ 504.47Y6T11T15+567.69Y6T14T15+437.12Y6T18T15+ 1188.49Y6T20T15+157.45Y6T18+882.52Y6T1T18+ 657.45Y6T2T18+487.45Y6T5T18+516.57Y6T7T18+ $325.05 Y 6 \mathrm{~T} 9 \mathrm{~T} 18+571.26 \mathrm{Y} 6 \mathrm{~T} 10 \mathrm{~T} 18+435.48 \mathrm{Y} 6 \mathrm{~T} 11 \mathrm{~T} 18+$ 675.31Y6T14T18+519.41Y6T15T18+1144.02Y6T20T18+ 978.63Y6T20+544.52Y6T2T20+579.29Y6T14T20+ 857.81Y6T15T20+444.48Y6T18T20+399.79Y7T6+ 612.26Y7T10T6+603.14Y7T14T6+397.16Y7T18T6+ 671.05Y7T10+1131.27Y7T6T10+446.98Y7T14T10+ 453.32Y7T18T10+381.88Y7T19T10+250.81Y7T14+ 801.86Y7T6T14+357.45Y7T10T14+548.09Y7T18T14+ 433.47Y7T19T14+235.66Y7T18+537.97Y7T6T18+ 395.53Y7T10 T18+537.79Y7T14T18+368.18Y7T19T18+
322.11Y7T19+524.21Y7T10T19+620.43Y7T14T19+ $510.58 \mathrm{Y} 7 \mathrm{~T} 18 \mathrm{~T} 19+426.09 \mathrm{Y} 8 \mathrm{~T} 14+478.84 \mathrm{Y} 8 \mathrm{~T} 15 \mathrm{~T} 14+$ 841.52Y8T18T14+435.34Y8T22T14+1113.68Y8T15+ 534.72Y8T14T15+1218.89Y8T18T15+494.01Y8T22T15+ $1890.00 \mathrm{Y} 8 \mathrm{~T} 18+659.15 \mathrm{Y} 8 \mathrm{~T} 14 \mathrm{~T} 18+783.54 \mathrm{Y} 8 \mathrm{~T} 15 \mathrm{~T} 18+$ $525.99 \mathrm{Y} 8 \mathrm{~T} 22 \mathrm{~T} 18+421.84 \mathrm{Y} 8 \mathrm{~T} 22+538.88 \mathrm{Y} 8 \mathrm{~T} 14 \mathrm{~T} 22+$ $605.89 \mathrm{Y} 8 \mathrm{~T} 15 \mathrm{~T} 22+938.68 \mathrm{Y} 8 \mathrm{~T} 18 \mathrm{~T} 22+1059.47 \mathrm{Y} 9 \mathrm{~T} 3+$ 752.02Y9T11 T3+552.85Y9T14T3+371.25Y9T18T3+ 828.20Y9T23T3+352.02Y9T6+569.24Y9T11T6+ 621.33Y9T14T6+440.15Y9T15T6+275.46Y9T18T6+ $3195.00 \mathrm{Y} 9 \mathrm{~T} 11+1177.64 \mathrm{Y} 9 \mathrm{~T} 3 \mathrm{~T} 11+936.92 \mathrm{Y} 9 \mathrm{~T} 6 \mathrm{~T} 11+$ 491.65Y9T14T11+1601.01Y9T15T11+1622.06Y9T16T11+ 315.27Y9T18T11+2499.11Y9T22T11+271.34Y9T14+ 491.23Y9T3T14+693.37Y9T6T14+349.02Y9T11T14+ 413.91Y9T15T14+646.97Y9T16T14+419.60Y9T18T14+ 448.57Y9T22T14+611.20Y9T23T14+648.70Y9T15+ 762.00Y9T6T15+468.40Y9T11T15+467.81Y9T14T15+ 476.67Y9T16T15+401.30Y9T18T15+578.64Y9T22T15+ 1042.11Y9T16+850.74Y9T11T16+798.51Y9T14T16+ 1021.37Y9T15T16+359.53Y9T18T16+813.20Y9T23T16+ 116.18Y9T18+541.16Y9T3T18+462.38Y9T6T18+ 323.03Y9T11 T18+565.13Y9T14T18+423.25Y9T15T18+ 509.27Y9T16T18+491.19Y9T22T18+461.05Y9T23T18+ 602.11Y9T22+1171.83Y9T11T22+479.44Y9T14T22+ 669.01Y9T15T22+421.03Y9T18T22+504.94Y9T23T22+ 971.74Y9T23+908.81Y9T3T23+866.89Y9T14T23+ 915.71Y9T16 T23+464.65Y9T18T23+551.01Y9T22T23+ 425.52Y10T6+664.03Y10T7T6+568.42Y10T14T6+ 414.25Y10T18T6+671.05Y10T7+882.35Y10T6T7+ 469.32Y10T14T7+520.52Y10T18T7+515.53Y10T19T7+ 226.40Y10T14+687.86Y10T6T14+367.58Y10T7T14+ 551.50Y10T1814+481.65Y10T1914+259.30Y10T18+ 463.52Y10T6T18+373.13Y10T7T18+517.73Y10T1418+ 417.27Y10T1918+224.42Y10T19+345.68Y10T7T19+ $652.80 \mathrm{Y} 10 \mathrm{~T} 1419+451.50 \mathrm{Y} 10 \mathrm{~T} 1819+260.69 \mathrm{Y} 11 \mathrm{~T} 3+$ 460.65Y11T9T3+605.53Y11T14T3+483.33Y11T18T3+ 465.58Y11T21T3+786.32Y11 T6+722.71Y11T9T6+ 617.12Y11T14T6+848.00Y11T15T6+365.78Y11T18T6+ 2125.00Y11T9+1196.25Y11T3T9+922.11Y11T6T9+ $500.38 \mathrm{Y} 11 \mathrm{~T} 14 \mathrm{~T} 9+1813.52 \mathrm{Y} 11 \mathrm{~T} 15 \mathrm{~T} 9+1155.07 \mathrm{Y} 11 \mathrm{~T} 16 \mathrm{~T} 9+$ 331.06Y11T18T9+2422.32Y11T22T9+287.26Y11T14+ 497.24Y11T3T14+696.75Y11T6T14+411.25Y11T9T14+ 396.07Y11T1514+608.30Y11T1614+510.42Y11T1814+ 481.55Y11T2214+1569.47Y11T15+983.83Y11T6T15+ 1030.31Y11T9T15+442.10Y11T1415+978.25Y11T1615+ 481.18Y11T1815+1514.10Y11T2215+442.63Y11T16+ 637.14Y11T9T16+726.58Y11T1416+857.19Y11T1516+ 488.21Y11T1816+340.00Y11T17+495.39Y11T1817+ 912.19Y11T2117+210.02Y11T18+474.73Y11T3T18+ $460.92 \mathrm{Y} 11 \mathrm{~T} 6 \mathrm{~T} 18+241.84 \mathrm{Y} 11 \mathrm{~T} 9 \mathrm{~T} 18+503.19 \mathrm{Y} 11 \mathrm{~T} 1418+$ 401.30Y11T1518+465.90Y11T1618+437.61Y11T1718+ $592.53 \mathrm{Y} 11 \mathrm{~T} 2118+442.70 \mathrm{Y} 11 \mathrm{~T} 2218+3390.00 \mathrm{Y} 11 \mathrm{~T} 21+$ $524.24 \mathrm{Y} 11 \mathrm{~T} 3 \mathrm{~T} 21+2452.97 \mathrm{Y} 11 \mathrm{~T} 1721+601.49 \mathrm{Y} 11 \mathrm{~T} 1821+$ $3256.78 \mathrm{Y} 11 \mathrm{~T} 2221+2602.63 \mathrm{Y} 11 \mathrm{~T} 22+911.40 \mathrm{Y} 11 \mathrm{~T} 9 \mathrm{~T} 22+$ 493.83Y11T1422+1034.88Y11T1522+520.67Y11T1822+ 1537.85Y11T2122+180.78Y12T18+423.90Y13T14+ 490.49Y13T1814+177.66Y13T18+670.82Y13T1418+ $168.51 \mathrm{Y} 14 \mathrm{~T} 1+285.61 \mathrm{Y} 14 \mathrm{~T} 5 \mathrm{~T} 1+1119.08 \mathrm{Y} 14 \mathrm{~T} 6 \mathrm{~T} 1+$ $592.96 \mathrm{Y} 14 \mathrm{~T} 18 \mathrm{~T} 1+214.31 \mathrm{Y} 14 \mathrm{~T} 2+716.04 \mathrm{Y} 14 \mathrm{~T} 6 \mathrm{~T} 2+$ 706.00Y14T15T2+630.33Y14T18T2+478.16Y14T19T2+ 306.10Y14T20T2+947.45Y14T22T2+318.38Y14T3+ $432.56 \mathrm{Y} 14 \mathrm{~T} 9 \mathrm{~T} 3+348.26 \mathrm{Y}_{14 \mathrm{~T} 11 \mathrm{~T} 3+562.87 \mathrm{Y} 14 \mathrm{~T} 18 \mathrm{~T} 3+}+$ 516.67Y14T23T3+178.51Y14T5+258.14Y14T1T5+ 647.01Y14T6T5+504.15Y14T18T5+401.84Y14T24T5+ $360.08 \mathrm{Y} 14 \mathrm{~T} 6+817.46 \mathrm{Y} 14 \mathrm{~T} 1 \mathrm{~T} 6+597.12 \mathrm{Y} 14 \mathrm{~T} 2 \mathrm{~T} 6+$ 


\section{APPENDIX B : PROBLEM FORMULATION FILE EFFICIENT AIRLINE NETWORK}

499.19Y14T5T6+596.40Y14T7T6+519.72Y14T9T6+ 497.93Y14T10T6+451.10Y14T11 T6+395.68Y14T15T6+ 451.44Y14T18T6+1007.32Y14T20T6+217.05Y14T7+ 637.72Y14T6T7+351.10Y14T10T7+553.17Y14T18T7+ 472.56Y14T19T7+256.67Y14T8+483.82Y14T15T8+ 785.05Y14T18T8+472.10Y14T22T8+219.13Y14T9+ 535.82Y14T3T9+573.64Y14T6T9+295.93Y14T11T9+ 409.73Y14T15T9+561.36Y14T16T9+417.01Y14T18T9+ 417.71Y14T22T9+568.14Y14T23T9+206.48Y14T10+ 678.56Y14T6T10+346.65Y14T7T10+478.29Y14T1810+ 424.27Y14T1910+223.89Y14T11+528.77Y14T3T11+ $597.67 \mathrm{Y} 14 \mathrm{~T} 6 \mathrm{~T} 11+360.93 \mathrm{Y} 14 \mathrm{~T} 9 \mathrm{~T} 11+342.05 \mathrm{Y} 14 \mathrm{~T} 1511+$ 688.99Y14T1611+488.32Y14T1811+468.01Y14T2211+ 562.76Y14T13+488.94Y14T1813+160.37Y14T15+ 749.02Y14T2T15+665.42Y14T6T15+452.93Y14T8T15+ 422.80Y14T9T15+318.38Y14T1115+615.73Y14T1615+ 560.45Y14T1815+442.10Y14T2015+388.37Y14T2215+ $620.52 \mathrm{Y} 14 \mathrm{~T} 16+530.40 \mathrm{Y} 14 \mathrm{~T} 9 \mathrm{~T} 16+390.12 \mathrm{Y} 14 \mathrm{~T} 1116+$ 449.54Y14T1516+538.59Y14T1816+668.69Y14T2316+ 293.85Y14T18+361.60Y14T1T18+496.70Y14T2T18+ 591.99Y14T3T18+378.82Y14T5T18+501.17Y14T6T18+ 448.55Y14T7T18+595.96Y14T8T18+319.20Y14T9T18+ 447.47Y14T1018+339.65Y14T1118+476.45Y14T1318+ $369.32 \mathrm{Y} 14 \mathrm{~T} 1518+646.05 \mathrm{Y} 14 \mathrm{~T} 1618+530.47 \mathrm{Y} 14 \mathrm{~T} 1918+$ 400.31Y14T2018+453.17Y14T2218+571.38Y14T2318+ 420.08Y14T2418+353.15Y14T2518+443.26Y14T19+ 427.14Y14T2T19+377.45Y14T7T19+446.83Y14T1019+ 525.26Y14T1819+342.73Y14T20+418.73Y14T2T20+ 1363.97Y14T6T20+790.44Y14T1520+598.23Y14T1820+ 567.21Y14T2220+222.98Y14T22+930.21Y14T2T22+ 411.49Y14T8T22+456.29Y14T9T22+361.79Y14T1122+ $345.65 \mathrm{Y} 14 \mathrm{~T} 1522+576.77 \mathrm{Y} 14 \mathrm{~T} 1822+382.15 \mathrm{Y} 14 \mathrm{~T} 2022+$ 493.78Y14T2322+373.12Y14T23+620.88Y14T3T23+ $614.72 \mathrm{Y} 14 \mathrm{~T} 9 \mathrm{~T} 23+831.82 \mathrm{Y} 14 \mathrm{~T} 1623+579.43 \mathrm{Y} 14 \mathrm{~T} 1823+$ 423.88Y14T2223+432.41 Y14T24+364.05Y14T5T24+ 482.70Y14T1824+146.22Y14T25+544.06Y14T1825+ 937.24Y15T2+748.49Y15T6T2+375.54Y15T14T2+ 578.31Y15T18T2+896.87Y15T20T2+983.43Y15T22T2+ 240.35Y15T6+934.84Y15T2T6+595.62Y15T9T6+ 497.33Y15T11T6+515.73Y15T14T6+372.56Y15T18T6+ $1446.80 \mathrm{Y} 15 \mathrm{~T} 20 \mathrm{~T} 6+961.58 \mathrm{Y} 15 \mathrm{~T} 8+595.28 \mathrm{Y} 15 \mathrm{~T} 14 \mathrm{~T} 8+$ 972.75Y15T18T8+524.35Y15T22T8+669.09Y15T9+ 555.89 Y $15 \mathrm{~T} 6 \mathrm{~T} 9+389.45 \mathrm{Y} 15 \mathrm{~T} 11 \mathrm{~T} 9+407.94 \mathrm{Y} 15 \mathrm{~T} 14 \mathrm{~T} 9+$ 399.58Y15T16 T9+352.75Y15T18T9+548.21Y15T22T9+ 1191.58Y15T11+778.60Y15T6T11+1092.32Y15T9T11+ 379.57Y15T1411+1061.93Y15T1611+424.24Y15T1811+ 1433.03Y15T2211+167.51Y15T14+765.80Y15T2T14+ 630.85Y15T6T14+428.73Y15T8T14+465.93Y15T9T14+ $329.86 \mathrm{Y} 15 \mathrm{~T} 1114+615.65 \mathrm{Y} 15 \mathrm{~T} 1614+513.91 \mathrm{Y} 15 \mathrm{~T} 1814+$ $621.67 Y 15 \mathrm{~T} 2014+399.80 \mathrm{Y} 15 \mathrm{~T} 2214+1002.11 \mathrm{Y} 15 \mathrm{~T} 16+$ 1057.72Y15T9T16+890.88Y15T1116+699.29Y15T1416+ 472.00Y15T1816+208.05Y15T18+847.28Y15T2T18+ 405.07Y15T6T18+536.70Y15T8T18+334.14Y15T9T18+ $330.09 \mathrm{Y} 15 \mathrm{~T} 1118+460.34 \mathrm{Y} 15 \mathrm{~T} 1418+489.90 \mathrm{Y} 15 \mathrm{~T} 1618+$ 752.70Y15T2018+433.24Y15T2218+623.64Y15T20+ 1015.02Y15T2T20+1305.07Y15T6T20+335.84Y15T1420+ $526.23 \mathrm{Y} 15 \mathrm{~T} 1820+561.10 \mathrm{Y} 15 \mathrm{~T} 2220+267.81 \mathrm{Y} 15 \mathrm{~T} 22+$ 1428.08Y15T2T22+399.17Y 15T8T22+595.91Y15T9T22+ $500.62 \mathrm{Y} 15 \mathrm{~T} 1122+386.43 \mathrm{Y} 15 \mathrm{~T} 1422+518.52 \mathrm{Y} 15 \mathrm{~T} 1822+$ 928.54Y15T2022+1016.84Y16T9+1070.88Y16 T11T9+ 847.27Y16T14T9+1219.82Y16T15T9+419.75Y16T18T9+ 996.32Y16T23T9+841.32Y16T11+643.23Y16T9T11+ 756.64Y16T1411+852.63Y16T1511+540.76Y16T1811+ 736.87Y16T14+578.54Y16T9T14+636.35Y16T1114+ $550.49 \mathrm{Y} 16 \mathrm{~T} 1514+606.56 \mathrm{Y} 16 \mathrm{~T} 1814+787.80 \mathrm{Y} 16 \mathrm{~T} 2314+$
1366.32Y16T15+1028.73Y16T9T15+1345.61Y16T1115+ 801.44Y16T1415+572.89Y16T1815+310.81Y16 T18+ $301.42 \mathrm{Y} 16 \mathrm{~T} 9 \mathrm{~T} 18+562.08 \mathrm{Y} 16 \mathrm{~T} 1118+720.73 \mathrm{Y} 16 \mathrm{~T} 1418+$ 477.63Y16T1518+424.25Y16T2318+982.11Y16T23+ $1040.56 \mathrm{Y} 16 \mathrm{~T} 9 \mathrm{~T} 23+1255.34 \mathrm{Y} 16 \mathrm{~T} 1423+654.11 \mathrm{Y} 16 \mathrm{~T} 1823+$ $11280.00 \mathrm{Y} 17 \mathrm{~T} 4+572.46 \mathrm{Y} 17 \mathrm{~T} 18 \mathrm{~T} 4+500.00 \mathrm{Y} 17 \mathrm{~T} 11+$ 513.74Y17T1811+1048.22Y17T2111+397.27Y17T18+ 536.58Y17T4T18+387.67Y17T1118+869.05Y17T2118+ $3690.00 \mathrm{Y} 17 \mathrm{~T} 21+1834.72 \mathrm{Y} 17 \mathrm{~T} 1121+762.86 \mathrm{Y} 17 \mathrm{~T} 1821+$ 272.41Y18T1+305.10Y18T5T1+868.50Y $18 \mathrm{~T} 6 \mathrm{~T} 1+$ 461.25Y $18 \mathrm{~T} 14 \mathrm{~T} 1+341.05 \mathrm{Y} 18 \mathrm{~T} 2+532.06 \mathrm{Y} 18 \mathrm{~T} 6 \mathrm{~T} 2+$ $507.68 \mathrm{Y} 18 \mathrm{~T} 14 \mathrm{~T} 2+807.24 \mathrm{Y} 18 \mathrm{~T} 15 \mathrm{~T} 2+371.01 \mathrm{Y} 18 \mathrm{~T} 19 \mathrm{~T} 2+$ 457.43Y $18 \mathrm{~T} 20 \mathrm{~T} 2+1016.79 \mathrm{Y} 18 \mathrm{~T} 22 \mathrm{~T} 2+265.48 \mathrm{Y} 18 \mathrm{~T} 3+$ $325.14 \mathrm{Y} 18 \mathrm{~T} 9 \mathrm{~T} 3+359.30 \mathrm{Y} 18 \mathrm{~T} 11 \mathrm{~T} 3+600.11 \mathrm{Y} 18 \mathrm{~T} 14 \mathrm{~T} 3+$ 493.11Y18T21T3+408.48Y18T23T3+181.06Y18T4+ 468.54Y18T17T4+214.63Y18T5+352.14Y18T1T5+ 459.17Y18T6T5+486.28Y18T14T5+305.82Y18T24T5+ 156.44Y18T6+925.31Y18T1T6+726.98Y18T2T6+ 534.22Y18T5T6+621.91Y18T7T6+420.04Y18T9T6+ 484.53Y18T10T6+481.21Y18T11T6+648.04Y18T14T6+ $502.36 \mathrm{Y} 18 \mathrm{~T} 15 \mathrm{~T} 6+1166.92 \mathrm{Y} 18 \mathrm{~T} 20 \mathrm{~T} 6+270.86 \mathrm{Y} 18 \mathrm{~T} 7+$ $464.59 \mathrm{Y} 18 \mathrm{~T} 6 \mathrm{~T} 7+325.78 \mathrm{Y} 18 \mathrm{~T} 10 \mathrm{~T} 7+535.70 \mathrm{Y} 18 \mathrm{~T} 14 \mathrm{~T} 7+$ $343.45 \mathrm{Y} 18 \mathrm{~T} 19 \mathrm{~T} 7+1492.11 \mathrm{Y} 18 \mathrm{~T} 8+736.03 \mathrm{Y} 18 \mathrm{~T} 14 \mathrm{~T} 8+$ 754.68Y18T15T8+587.59Y18T22T8+122.45Y18T9+ 497.78Y18T3T9+373.79Y18T6T9+319.13Y18T11 T9+ $525.00 \mathrm{Y} 18 \mathrm{~T} 14 \mathrm{~T} 9+494.46 \mathrm{Y} 18 \mathrm{~T} 15 \mathrm{~T} 9+439.27 \mathrm{Y} 18 \mathrm{~T} 16 \mathrm{~T} 9+$ 479.55Y18T22T9+494.65Y18T23T9+193.17Y18T10+ 490.15Y18T6T10+375.19Y18T7T10+505.03Y18T1410+ $322.70 \mathrm{Y} 18 \mathrm{~T} 1910+200.04 \mathrm{Y} 18 \mathrm{~T} 11+450.68 \mathrm{Y} 18 \mathrm{~T} 3 \mathrm{~T} 11+$ 415.48Y18T6T11+251.65Y18T9T11+468.49Y18T1411+ 415.02Y18T1511+580.24Y18T1611+405.46Y18T1711+ 538.70Y18T2111+464.42Y18T2211+173.87Y18T12+ $178.88 \mathrm{Y} 18 \mathrm{~T} 13+706.77 \mathrm{Y} 18 \mathrm{~T} 1413+307.90 \mathrm{Y} 18 \mathrm{~T} 14+$ 407.98Y18T1T14+564.93Y18T2 T14+532.54Y18T3T14+ $382.64 \mathrm{Y} 18 \mathrm{~T} 5 \mathrm{~T} 14+540.02 \mathrm{Y} 18 \mathrm{~T} 6 \mathrm{~T} 14+480.37 \mathrm{Y} 18 \mathrm{~T} 7 \mathrm{~T} 14+$ 518.68Y18T8T14+375.69Y18T9T14+411.67Y18T1014+ 382.43Y18T1114+479.18Y18T1314+433.39Y18T1514+ 657.36Y18T1614+486.71Y18T1914+429.59Y18T2014+ 494.11Y18T2214+637.23Y18T2314+400.58Y18T2414+ 396.86Y18T2514+276.57Y18T15+898.98Y18T2T15+ 475.18Y18T6T15+504.95Y18T8T15+344.50Y18T9T15+ 361.00Y18T1115+466.64Y18T1415+507.73Y18T1615+ $599.92 \mathrm{Y} 18 \mathrm{~T} 2015+467.73 \mathrm{Y} 18 \mathrm{~T} 2215+254.99 \mathrm{Y} 18 \mathrm{~T} 16+$ $326.92 \mathrm{Y} 18 \mathrm{~T} 9 \mathrm{~T} 16+396.41 \mathrm{Y} 18 \mathrm{~T} 1116+667.45 \mathrm{Y} 18 \mathrm{~T} 1416+$ 478.57Y18T1516+401.15Y18T2316+236.42Y18T17+ 412.22Y18T4T17+382.98Y18T1117+644.92Y18T2117+ 339.13Y18T19+513.10Y18T2T19+423.30Y18T7T19+ $371.38 \mathrm{Y} 18 \mathrm{~T} 1019+664.59 \mathrm{Y} 18 \mathrm{~T} 1419+285.15 \mathrm{Y} 18 \mathrm{~T} 20+$ 494.84Y18T2T20+1079.27Y18T6T20+449.99Y18T1420+ 768.29Y18T1520+534.44Y18T2220+417.66Y18T21+ $451.92 \mathrm{Y} 18 \mathrm{~T} 3 \mathrm{~T} 21+396.98 \mathrm{Y} 18 \mathrm{~T} 1121+552.90 \mathrm{Y} 18 \mathrm{~T} 1721+$ $458.03 \mathrm{Y} 18 \mathrm{~T} 2221+318.21 \mathrm{Y} 18 \mathrm{~T} 22+1100.33 \mathrm{Y} 18 \mathrm{~T} 2 \mathrm{~T} 22+$ 459.06 Y18T8T22+353.25Y18T9T22+393.13Y18T1122+ 528.27Y18T1422+462.94Y18T1522+517.06Y18T2022+ $538.12 \mathrm{Y} 18 \mathrm{~T} 2122+435.94 \mathrm{Y} 18 \mathrm{~T} 2322+377.01 \mathrm{Y} 18 \mathrm{~T} 23+$ $480.30 \mathrm{Y} 18 \mathrm{~T} 3 \mathrm{~T} 23+398.24 \mathrm{Y} 18 \mathrm{~T} 9 \mathrm{~T} 23+661.37 \mathrm{Y} 18 \mathrm{~T} 1423+$ $476.98 \mathrm{Y} 18 \mathrm{~T} 1623+446.63 \mathrm{Y} 18 \mathrm{~T} 2223+237.66 \mathrm{Y} 18 \mathrm{~T} 24+$ $370.42 \mathrm{Y} 18 \mathrm{~T} 5 \mathrm{~T} 24+568.62 \mathrm{Y} 18 \mathrm{~T} 1424+265.42 \mathrm{Y} 18 \mathrm{~T} 25+$ $440.87 \mathrm{Y} 18 \mathrm{~T} 1425+307.48 \mathrm{Y} 19 \mathrm{~T} 2+565.58 \mathrm{Y} 19 \mathrm{~T} 14 \mathrm{~T} 2+$ 691.00Y19T18T2+671.05Y19T7+502.67Y19T10T7+ 633.97Y19T14T7+681.39Y19T18T7+221.90Y19T10+ 331.17Y19T7T10+509.61Y19T1410+498.38Y19T1810+ 443.26Y19T14+438.32Y19T2T14+402.72Y19T7T14+ 367.91Y19T1014+616.84Y19T1814+250.00Y19T18+ 553.09Y19T2T18+459.07Y19T7T18+426.62Y19T1018+ 


\section{APPENDIX B : PROBLEM FORMULATION FILE EFFICIENT AIRLINE NETWORK}

$680.58 \mathrm{Y} 19 \mathrm{~T} 1418+220.39 \mathrm{Y} 20 \mathrm{~T} 2+1341.88 \mathrm{Y} 20 \mathrm{~T} 6 \mathrm{~T} 2+$ $377.39 \mathrm{Y} 20 \mathrm{~T} 14 \mathrm{~T} 2+935.52 \mathrm{Y} 20 \mathrm{~T} 15 \mathrm{~T} 2+628.96 \mathrm{Y} 20 \mathrm{~T} 18 \mathrm{~T} 2+$ $1180.54 \mathrm{Y} 20 \mathrm{~T} 22 \mathrm{~T} 2+978.63 \mathrm{Y} 20 \mathrm{~T} 6+590.34 \mathrm{Y} 20 \mathrm{~T} 2 \mathrm{~T} 6+$ $570.12 \mathrm{Y} 20 \mathrm{~T} 14 \mathrm{~T} 6+603.19 \mathrm{Y} 20 \mathrm{~T} 15 \mathrm{~T} 6+430.32 \mathrm{Y} 20 \mathrm{~T} 18 \mathrm{~T} 6+$ 776.32Y20T14+429.34Y20T2T14+1271.83Y20T6T14+ 491.42Y20T1514+599.28Y20T1814+871.46Y20T2214+ $538.62 \mathrm{Y} 20 \mathrm{~T} 15+891.44 \mathrm{Y} 20 \mathrm{~T} 2 \mathrm{~T} 15+1470.80 \mathrm{Y} 20 \mathrm{~T} 6 \mathrm{~T} 15+$ 474.83Y20T1415+557.22Y20T1815+716.40Y20T2215+ $299.03 \mathrm{Y} 20 \mathrm{~T} 18+468.04 \mathrm{Y} 20 \mathrm{~T} 2 \mathrm{~T} 18+1057.06 \mathrm{Y} 20 \mathrm{~T} 6 \mathrm{~T} 18+$ 452.42Y20T1418+524.63Y20T1518+497.37Y20T2218+ 1052.59Y20T22+1136.89Y20T2T22+523.68Y20 T1422+ 758.93Y20T1522+592.56Y20T1822+867.06Y21T3+ 1090.77Y21T11T3+706.92Y21T18T3+3048.57Y21T11+ 902.63Y21T3T11+2087.22Y21T1711+701.61Y21T1811+ $3328.08 \mathrm{Y} 21 \mathrm{~T} 2211+3330.00 \mathrm{Y} 21 \mathrm{~T} 17+1811.40 \mathrm{Y} 21 \mathrm{~T} 1117+$ 729.72Y21T1817+548.18Y21T18+506.38Y21T3T18+ 453.42Y21T1118+674.77Y21T1718+522.58Y21T2218+ 3465.00Y21T22+3619.54Y21T1122+825.22Y21T1822+ 1927.89Y22T2+447.21 Y22T14T2+767.35Y22T15T2+ $577.90 \mathrm{Y} 22 \mathrm{~T} 18 \mathrm{~T} 2+651.78 \mathrm{Y} 22 \mathrm{~T} 20 \mathrm{~T} 2+411.32 \mathrm{Y} 22 \mathrm{~T} 8+$ 603.29Y22T14T8+521.62Y22T15 T8+738.22Y22T18T8+ 514.47Y22T9+1078.37Y22T11T9+446.33Y22T14T9+ 649.10Y22T15T9+359.95Y22T18T9+485.28Y22T23T9+ 2365.79Y22T11+898.63Y22T9T11+450.87Y22T1411+ 808.5TY22T1511+442.29Y22T1811+1347.17Y22T2111+ 240.64Y22T14+963.70Y22T2T14+406.95Y22T8T14+ 462.67Y22T9T14+376.17Y22T1114+352.57Y22T1514+ 554.98Y22T $1814+413.06 \mathrm{Y} 22 \mathrm{~T} 2014+510.55 \mathrm{Y} 22 \mathrm{~T} 2314+$ $225.91 \mathrm{Y} 22 \mathrm{~T} 15+1511.89 \mathrm{Y} 22 \mathrm{~T} 2 \mathrm{~T} 15+439.53 \mathrm{Y} 22 \mathrm{~T} 8 \mathrm{~T} 15+$ 485.49Y22T9T15+457.31Y22T1115+397.43Y22T1415+ $548.29 \mathrm{Y} 22 \mathrm{~T} 1815+643.65 \mathrm{Y} 22 \mathrm{~T} 2015+248.79 \mathrm{Y} 22 \mathrm{~T} 18+$ 1033.12Y22T2T18+524.71Y22T8T18+310.99Y22T9T18+ 386.04Y22T1118+508.58Y22T $1418+401.45 \mathrm{Y} 22 \mathrm{~T} 1518+$ $515.50 \mathrm{Y} 22 \mathrm{~T} 2018+581.90 \mathrm{Y} 22 \mathrm{~T} 2118+360.29 \mathrm{Y} 22 \mathrm{~T} 2318+$ $635.94 \mathrm{Y} 22 \mathrm{~T} 20+1290.19 \mathrm{Y} 22 \mathrm{~T} 2 \mathrm{~T} 20+380.67 \mathrm{Y} 22 \mathrm{~T} 1420+$ $813.49 \mathrm{Y} 22 \mathrm{~T} 1520+570.34 \mathrm{Y} 22 \mathrm{~T} 1820+2193.33 \mathrm{Y} 22 \mathrm{~T} 21+$ $3338.91 \mathrm{Y} 22 \mathrm{~T} 1121+634.97 \mathrm{Y} 22 \mathrm{~T} 1821+245.69 \mathrm{Y} 22 \mathrm{~T} 23+$ 479.57Y22 T9T23+698.12Y22T1423+593.63Y22T1823+ $592.11 \mathrm{Y} 23 \mathrm{~T} 3+1035.00 \mathrm{Y} 23 \mathrm{~T} 9 \mathrm{~T} 3+909.83 \mathrm{Y} 23 \mathrm{~T} 14 \mathrm{~T} 3+$ 489.10Y23T18T3+1010.53Y23T9+927.63Y23T3T9+ 837.84Y23T14T9+789.47Y23 T16 $9+352.83 \mathrm{Y} 23 \mathrm{~T} 18 \mathrm{~T} 9+$ 515.71Y23T22T9+373.12Y23T14+514.17Y23T3T14+ $629.40 \mathrm{Y} 23 \mathrm{~T} 9 \mathrm{~T} 14+788.16 \mathrm{Y} 23 \mathrm{~T} 1614+550.10 \mathrm{Y} 23 \mathrm{~T} 1814+$ $400.85 \mathrm{Y} 23 \mathrm{~T} 2214+562.11 \mathrm{Y} 23 \mathrm{~T} 16+1026.32 \mathrm{Y} 23 \mathrm{~T} 9 \mathrm{~T} 16+$ $1155.49 \mathrm{Y} 23 \mathrm{~T} 1416+477.38 \mathrm{Y} 23 \mathrm{~T} 1816+248.48 \mathrm{Y} 23 \mathrm{~T} 18+$ 464.63Y23T3T18+309.20Y23T9T18+686.03Y23T1418+ $430.18 Y 23 \mathrm{~T} 1618+431.70 \mathrm{Y} 23 \mathrm{~T} 2218+229.31 \mathrm{Y} 23 \mathrm{~T} 22+$ 487.68Y23T9T22+681.59Y23T1422+576.43Y23T1822+ $1531.43 \mathrm{Y} 24 \mathrm{~T} 5+564.57 \mathrm{Y} 24 \mathrm{~T} 14 \mathrm{~T} 5+454.33 \mathrm{Y} 24 \mathrm{~T} 18 \mathrm{~T} 5+$ 386.90Y24T14+363.34Y24T5T14+503.16Y24T1814+ $250.19 \mathrm{Y} 24 \mathrm{~T} 18+369.29 \mathrm{Y} 24 \mathrm{~T} 5 \mathrm{~T} 18+543.90 \mathrm{Y} 24 \mathrm{~T} 1418+$ 147.89Y25T14+533.28Y25T1814+286.75Y25T18+ 443.57Y25T1418

ST

Y1T6-Y1T6T5 > $>0$

Y6T5-Y1T6T5 $>=0$

Y1T14-Y1T14T5 $>=0$

Y14T5-Y1T14T5 > $=0$

Y1T18-Y1T18T5 $>=0$

Y18T5-Y1T18T5 > =0

Y1T5-Y1T5T6 > $=0$

Y5T6-Y1T5T6 > $>0$

Y1T14-Y1T14T6 > $=0$

Y14T6-Y1T14T6 > $=0$

Y1T18-Y1T18T6 > $=0$
Y18T6-Y1T18T6>=0

Y1T5-Y1T5T14 $>=0$

Y5T14-Y1T5T14 $>=0$

Y1T6-Y1T6T14 >= 0

Y6T14-Y1T6T14 >= 0

Y1T18-Y1T18T14>=0

Y18T14-Y1T18T14 > $=0$

Y1T5-Y1T5T18 > $=0$

Y5T18-Y1T5T18 > $=0$

Y1T6-Y1T6T18 > $=0$

Y6T18-Y1T6T18 > 0

Y1T14-Y1T14T18 $>0$

Y14T18-Y1T14T18 $>=0$

Y2T14-Y2T14T6 $>=0$

Y14T6-Y2T14T6> $>0$

Y2T15-Y2T15T6 $>=0$

Y15T6-Y2T15T6>=0

Y2T18-Y2T18T6 > $=0$

Y18T6-Y2T18T6 > $=0$

Y2T20-Y2T20T6 > $=0$

Y20T6-Y2T20T6 > 0

Y2T6-Y2T6T14 $>=0$

Y6T14-Y2T6T14 $>=0$

Y2T15-Y2T15T14 > $=0$

Y15T14-Y2T15T14 > $=0$

$Y 2 T 18-Y 2 T 18 T 14>0$

$\mathrm{Y} 18 \mathrm{~T} 14-\mathrm{Y} 2 \mathrm{~T} 18 \mathrm{~T} 14>=0$

Y2T19-Y2T19T14 $>=0$

Y19T14-Y2T19T14 $>=0$

Y2T20-Y2T20T14 $>=0$

Y20T14-Y2T20T14 > $=0$

Y2T22-Y2T22T14 > 0

$\mathrm{Y} 22 \mathrm{~T} 14-\mathrm{Y} 2 \mathrm{~T} 2 \mathrm{~T} 14>=0$

Y2T6-Y2T6T15 $>=0$

Y6T15-Y2T6T15 > $=0$

Y2T14-Y2T14T15 > = 0

Y14T15-Y2T14T15 $>0$

Y2T18-Y2T18T15 $>=0$

$\mathrm{Y} 18 \mathrm{~T} 15-\mathrm{Y} 2 \mathrm{~T} 18 \mathrm{~T} 15>=0$

Y2T20-Y2T20T15 $>=0$

Y20T15-Y2T20T15 > = 0

Y2T22-Y2T22T15 $>=0$

Y22T15-Y2T2T15 $>=0$

Y2T6-Y2T6T18 $>=0$

Y6T18-Y2T6T18 $>=0$

Y2T14-Y2T14T18 > $=0$

Y14T18-Y2T14T18 > $=0$

Y2T15-Y2T15T18 $>=0$

Y15T18-Y2T15T18 $>=0$

Y2T19-Y2T19T18 $>=0$

Y19T18-Y2T19T18 > $=0$

Y2T20-Y2T20T18 $>=0$

Y20T18-Y2T20T18 > $=0$

$\mathrm{Y} 2 \mathrm{~T} 22-\mathrm{Y} 2 \mathrm{~T} 22 \mathrm{~T} 18>0$

Y22T18-Y2T22T18 > $=0$

Y2T14-Y2T14T19 > $=0$

Y14T19-Y2T14T19 > 0

Y2T18-Y2T18T19 > $=0$

Y18T19-Y2T18T19 >= 0

Y2T6-Y2T6T20 > $=0$

Y6T20-Y2T6T20 $>=0$

Y2T14-Y2T14T20 $>=0$

Y14T20-Y2T14T20 > $=0$

Y2T15-Y2T15T20 $>=0$

Y15T20-Y2T15T20 > $=0$ 


\section{APPENDIX B : PROBLEM FORMULATION FILE} EFFICIENT AIRLINE NETWORK

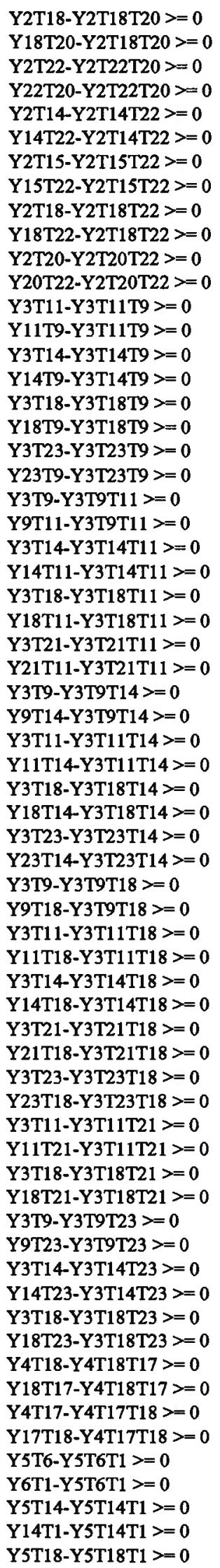

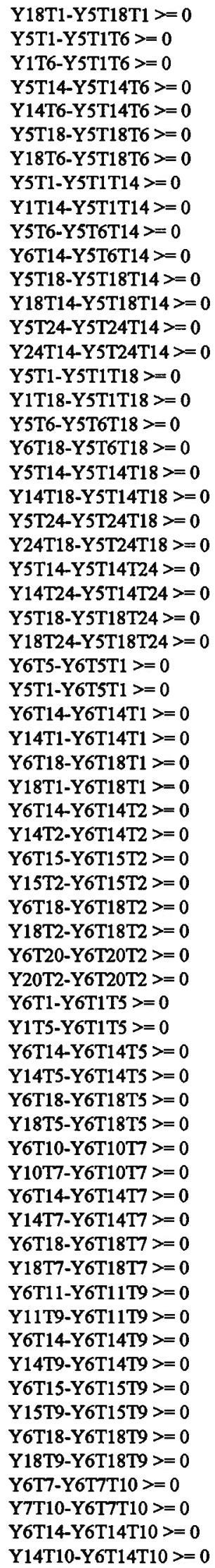


APPENDIX B : PROBLEM FORMULATION FILE EFFICIENT AIRLINE NETWORK

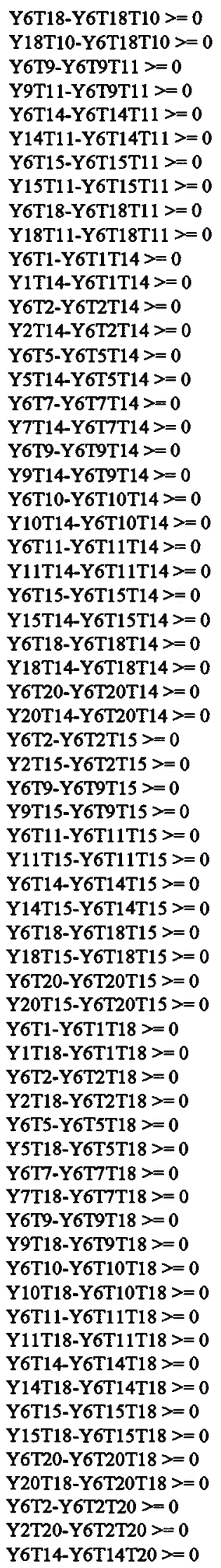

$\mathrm{Y} 14 \mathrm{~T} 20-\mathrm{Y} 6 \mathrm{~T} 14 \mathrm{~T} 20>=0$

Y6T15-Y6T15T20 > $=0$

Y15T20-Y6T15T20 > $=0$

Y6T18-Y6T18T20 > 0

$\mathrm{Y} 18 \mathrm{~T} 20-\mathrm{Y} 6 \mathrm{~T} 18 \mathrm{~T} 20>=0$

Y7T10-Y7T10T6 $>=0$

Y10T6-Y7T10T6 $>=0$

Y7T14-Y7T14T6 > $=0$

Y14T6-Y7T14T6 > $=0$

Y7T18-Y7T18T6 > $=0$

$\mathrm{Y} 18 \mathrm{~T} 6-\mathrm{Y} 7 \mathrm{~T} 18 \mathrm{~T} 6>=0$

Y7T6-Y7T6T10 $>=0$

Y6T10-Y7T6T10 > $=0$

Y7T14-Y7T14T10 > $=0$

Y14T10-Y7T14T10 > $=0$

Y7T18-Y7T18T10 $>=0$

Y18T10-Y7T18T10 $>=0$

Y7T19-Y7T19T10> $>0$

Y19T10-Y7T19T10 > $=0$

Y7T6-Y7T6T14 > =0

Y6T14-Y7T6T14 > $=0$

Y7T10-Y7T10T14 > $=0$

Y10T14-Y7T10T14 $>=0$

Y7T18-Y7T18T14 > $=0$

$Y 18 T 14-Y 7 T 18 T 14>=0$

Y7T19-Y7T19T14 $>=0$

Y19T14-Y7T19T14 > $=0$

Y7T6-Y7T6T18 > $=0$

Y6T18-Y7T6T18 >= 0

Y7T10-Y7T10T18 > $=0$

Y10T18-Y7T10T18 > $=0$

Y7T14-Y7T14T18 $>=0$

Y14T18-Y7T14T18 $>=0$

Y7T19-Y7T19T18 > $=0$

$Y 19 T 18-Y 7 T 19 T 18>=0$

Y7T10-Y7T10T19 > $=0$

Y10T19-Y7T10T19>=0

Y7T14-Y7T14T19 > $=0$

Y14T19-Y7T14T19 > $=0$

Y7T18-Y7T18T19 > $=0$

Y18T19-Y7T18T19 > $=0$

Y8T15-Y8T15T14 $>=0$

Y15T14-Y $8 T 15 \mathrm{~T} 14>=0$

Y8T18-Y8T18T14 $>=0$

Y18T14-Y8T18T14 > $=0$

Y8T22-Y8T22T14 > $=0$

Y22T14-Y8T22T14 > $=0$

Y8T14-Y8T14T15 > $=0$

$Y 14 T 15-Y 8 T 14 T 15>=0$

Y8T18-Y8T18T15 $>=0$

Y18T15-Y8T18T15 > $=0$ Y8T22-Y8T22T15 > 0

$\mathrm{Y} 22 \mathrm{~T} 15-\mathrm{Y} 8 \mathrm{~T} 22 \mathrm{~T} 15>=0$

Y8T14-Y8T14T18 $>=0$

Y14T18-Y8T14T18 > $=0$

Y8T15-Y8T15T18 $>=0$

Y15T18-Y8T15T18 > $=0$

Y8T22-Y8T22T18 > $=0$

$\mathrm{Y} 22 \mathrm{~T} 18-\mathrm{Y} 8 \mathrm{~T} 22 \mathrm{~T} 18>=0$

Y8T14-Y8T14T22 > $=0$

Y14T22-Y8T14T22 > $=0$

Y8T15-Y8T15T22 > 0

Y15T22-Y8T15T22 > $=0$

Y8T18-Y8T18T22 $>=0$

Y18T22-Y8T18T22 > $=0$ 


\section{APPENDIX B : PROBLEM FORMULATION FILE EFFICIENT AIRLINE NETWORK}

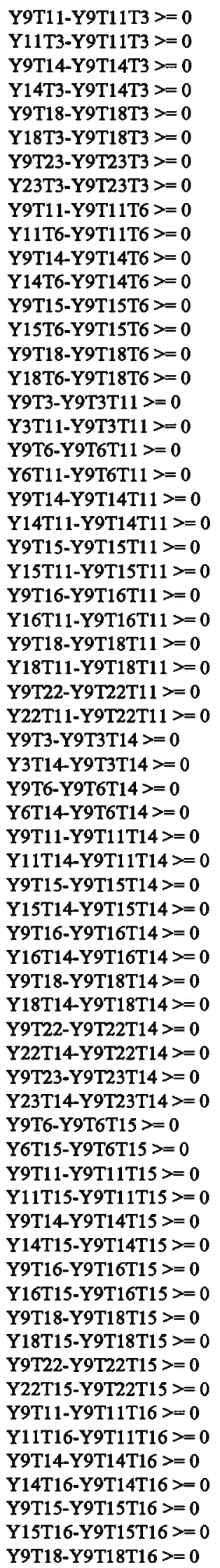

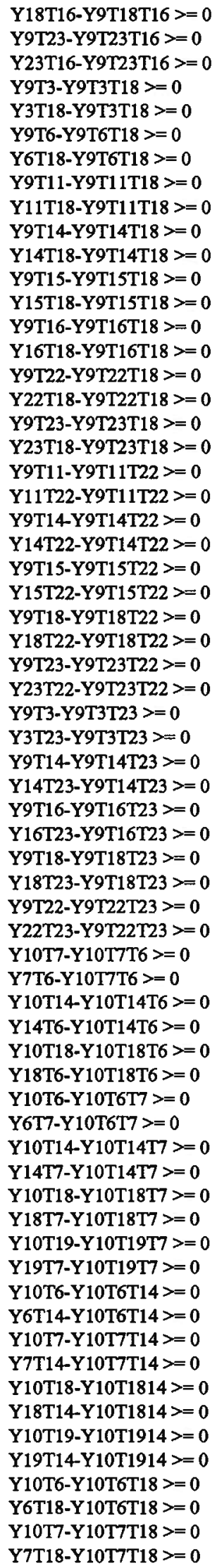


APPENDIX B : PROBLEM FORMULATION FILE EFFICIENT AIRLINE NETWORK

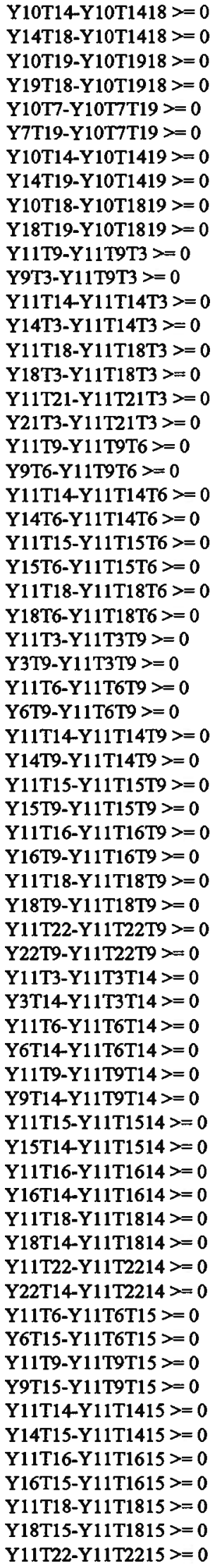

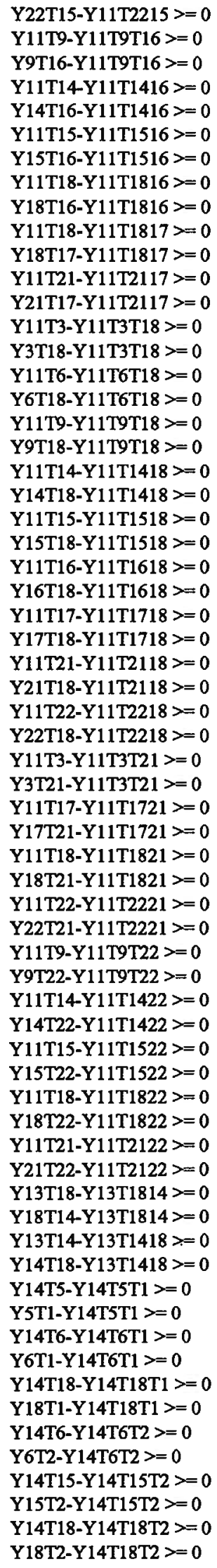


APPENDIX B : PROBLEM FORMULATION FILE EFFICIENT AIRLINE NETWORK

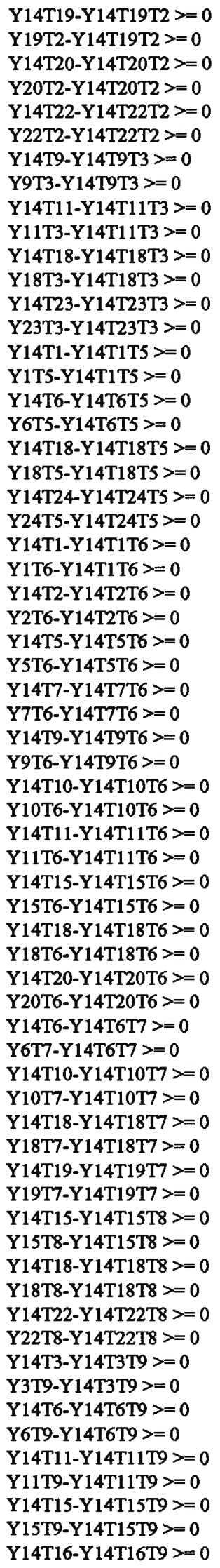

Y16T9-Y14T16T9

Y14T18-Y14T18T9 > $=0$

Y18T9-Y14T18T9>=0

$Y 14 T 22-Y 14 T 22 T 9>=0$

Y22T9-Y14T22T9> $>0$

Y14T23-Y14T23T9 $>=0$

Y23T9-Y14T23T9 $>=0$

Y14T6-Y14T6T10 > $=0$

Y6T10-Y14T6T10 > $=0$

$\mathrm{Y} 14 \mathrm{~T} 7-\mathrm{Y} 14 \mathrm{~T} 7 \mathrm{~T} 10>=0$

Y7T10-Y14T7T10 > $=0$

Y14T18-Y14T1810>=0

$Y 18 T 10-Y 14 T 1810>=0$

Y14T19-Y14T1910>=0

Y19T10-Y14T1910>=0

Y14T3-Y14T3T11 $>=0$

Y3T11-Y14T3T11 $>=0$

Y14T6-Y14T6T11 >=0

Y $6 \mathrm{~T} 11-\mathrm{Y} 14 \mathrm{~T} 6 \mathrm{~T} 11>=0$

Y14T9-Y14T9T11 > $=0$

Y9T11-Y14T9T11 >= 0

Y14T15-Y14T1511 > $=0$

Y15T11-Y14T1511 $>=0$

Y14T16-Y14T1611 > $=0$

Y16T11-Y14T1611 $>=0$

Y14T18-Y14T1811 > $=0$

$Y 18 T 11-Y 14 T 1811>=0$

$Y 14 T 22-Y 14 T 2211>=0$

Y22T11-Y14T2211 > $=0$

$Y 14 T 18-Y 14 T 1813>00$

Y18T13-Y14T1813>=0

$\mathrm{Y} 14 \mathrm{~T} 2-\mathrm{Y} 14 \mathrm{~T} 2 \mathrm{~T} 15>=0$

$\mathrm{Y} 2 \mathrm{~T} 15-\mathrm{Y} 14 \mathrm{~T} 2 \mathrm{~T} 15>=0$

Y14T6-Y14T6T15 $>=0$

Y6T15-Y14T6T15 $>=0$

$Y 14 T 8-Y 14 T 8 T 15>=0$

Y8T15-Y14T8T15 > $=0$

Y14T9-Y14T9T15 > $=0$

Y9T15-Y14T9T15 $>=0$

$Y 14 T 11-Y 14 T 1115>=0$

Y11T15-Y14T1115>=0

$Y 14 T 16-Y 14 T 1615>=0$

Y16T15-Y14T1615 >=0

$Y 14 T 18 \cdot Y 14 T 1815>=0$

$Y 18 T 15-Y 14 T 1815>=0$

$Y 14 T 20-Y 14 T 2015>=0$

Y20T15-Y14T2015 > $=0$

$Y 14 T 22-Y 14 T 2215>=0$

$Y 22 T 15-Y 14 T 2215>=0$

Y14T9-Y14T9T16 $>=0$

Y9T16-Y14T9T16 $>=0$

$\mathrm{Y} 14 \mathrm{~T} 11-\mathrm{Y} 14 \mathrm{~T} 1116>=0$

Y11T16-Y14T1116>=0

$Y 14 T 15-Y 14 T 1516>=0$

Y15T16-Y14T1516 > $=0$

$Y 14 T 18-Y 14 T 1816>=0$

$\mathrm{Y} 18 \mathrm{~T} 16-\mathrm{Y} 14 \mathrm{~T} 1816>=0$

$Y 14 T 23-Y 14 T 2316>=0$

Y23T16-Y14T2316>=0

Y14T1-Y14T1T18 > $=0$

$Y 1 T 18-Y 14 T 1 T 18>=0$

Y14T2-Y14T2T18 > $=0$

Y2T18-Y14T2T18 $>=0$

Y14T3-Y14T3T18 > $=0$

Y3T18-Y14T3T18 >= 0 
APPENDIX B : PROBLEM FORMULATION FILE EFFICIENT AIRLINE NETWORK

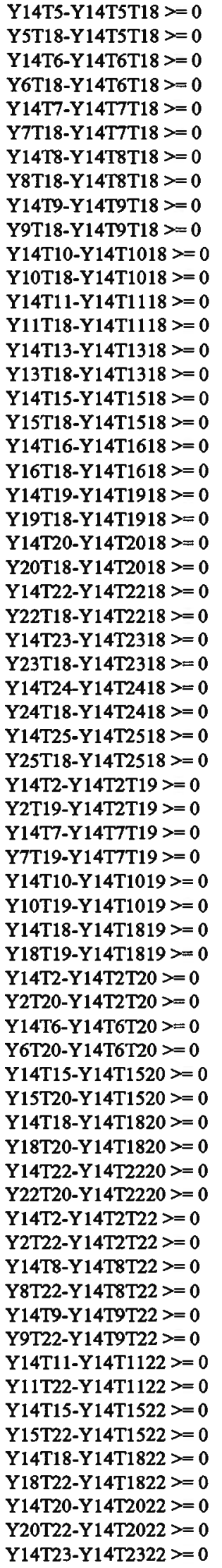

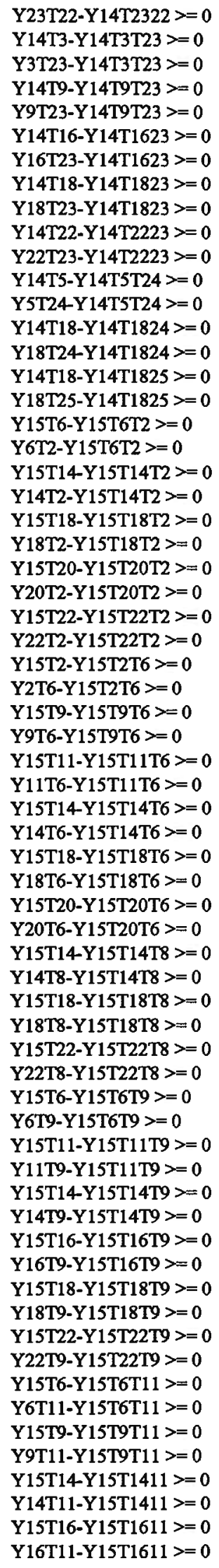


APPENDIX B : PROBLEM FORMULATION FILE EFFICIENT AIRLINE NETWORK

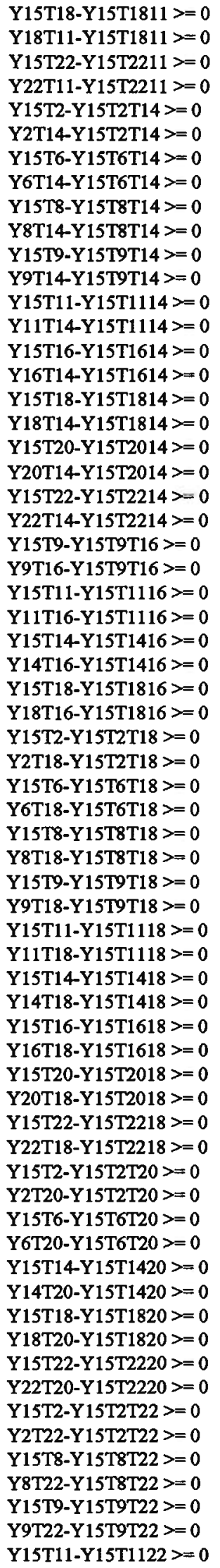

Y11T22-Y15T1122 > $=0$ $Y 15 T 14-Y 15 T 1422>=0$ $\mathrm{Y} 14 \mathrm{~T} 22-\mathrm{Y} 15 \mathrm{~T} 1422>=0$ $Y 15 T 18-Y 15 T 1822>=0$ Y18T22-Y15T1822 > $=0$ Y15T20-Y15T2022 > $=0$ Y20T22-Y15T2022 > $=0$ Y16T11-Y16T11T9 $>=0$ Y11T9-Y16T11T9 > $=0$ Y16T14-Y16T14T9 > $=0$ Y14T9-Y16T14T9 > $=0$ Y16T15-Y16T15T9 $>=0$ Y15T9-Y16T15T9 $>=0$ $\mathrm{Y} 16 \mathrm{~T} 18-\mathrm{Y} 16 \mathrm{~T} 18 \mathrm{~T} 9>=0$ Y18T9-Y16T18T9 > $=0$ Y16T23-Y16T23T9 $>=0$ Y23T9-Y16T23T9 > $=0$

Y16T9-Y16T9T11 > $=0$

Y9T11-Y16T9T11 $>=0$

Y16T14-Y16T1411 > $=0$ Y14T11-Y16T1411 >= 0 Y16T15-Y16T1511 > $=0$ Y15T11-Y16T1511 > $=0$ Y16T18-Y16T1811 >=0 Y18T11-Y16T1811 $>=0$ Y16T9-Y16T9T14 $>=0$ Y9T14Y16T9T14 $>=0$ Y16T11-Y16T1114 $>=0$ Y11T14-Y16T1114 $>=0$ $Y 16 \mathrm{~T} 15-\mathrm{Y} 16 \mathrm{~T} 1514>=0$ $Y 15 T 14-Y 16 T 1514>=0$ Y16T18-Y16T1814 > $=0$ $\mathrm{Y} 18 \mathrm{~T} 14-\mathrm{Y} 16 \mathrm{~T} 1814>=0$ Y16T23-Y16T2314 > $=0$ $Y 23 \mathrm{~T} 14-\mathrm{Y} 16 \mathrm{~T} 2314>=0$ Y16T9-Y16T9T15 > $=0$ Y9T15-Y16T9T15 > $=0$ Y16T11-Y16T1115 >= 0 Y11T15-Y16T1115 > $=0$ Y16T14-Y16T1415 >=0 Y14T15-Y16T1415 $>=0$ Y16T18-Y16T1815 > $=0$ Y18T15-Y16T1815>=0 Y16T9-Y16T9T18 >= 0 Y9T18-Y16T9T18 $>=0$ Y16T11-Y16T1118>=0 $Y 11 T 18-Y 16 \mathrm{~T} 1118>=0$ Y16T14-Y16T1418 > $=0$ $Y 14 T 18-Y 16 T 1418>=0$ $\mathrm{Y} 16 \mathrm{~T} 15-\mathrm{Y} 16 \mathrm{~T} 1518>=0$ $Y 15 T 18-Y 16 T 1518>=0$ Y16T23-Y16T2318 > $=0$ Y23T18-Y16T2318 >=0 Y16T9-Y16T9T23 > $=0$ Y9T23-Y16T9T23 > $=0$ Y16T14-Y16T1423 > $=0$ $Y 14 T 23-Y 16 T 1423>=0$ Y16T18-Y16T1823 $>=0$ Y18T23-Y16T1823 $>=0$ Y17T18-Y17T18T4 $>=0$ Y18T4-Y17T18T4 > $=0$ Y17T18-Y17T1811 > $=0$ Y18T11-Y17T1811 > $=0$ Y17T21-Y17T2111 $>=0$ Y21T11-Y17T2111 $>=0$ 


\section{APPENDIX B : PROBLEM FORMULATION FILE EFFICIENT AIRLINE NETWORK}

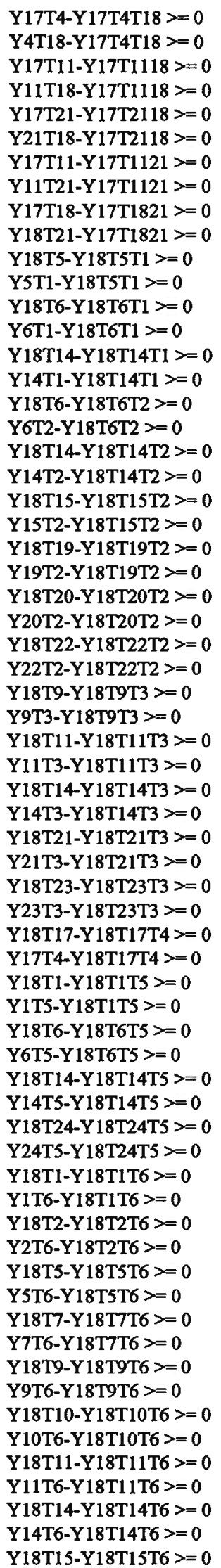

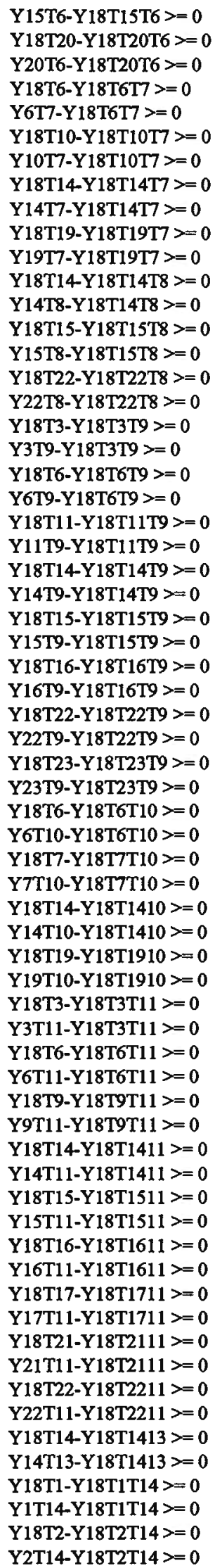


APPENDIX B : PROBLEM FORMULATION FILE EFFICIENT AIRLINE NETWORK

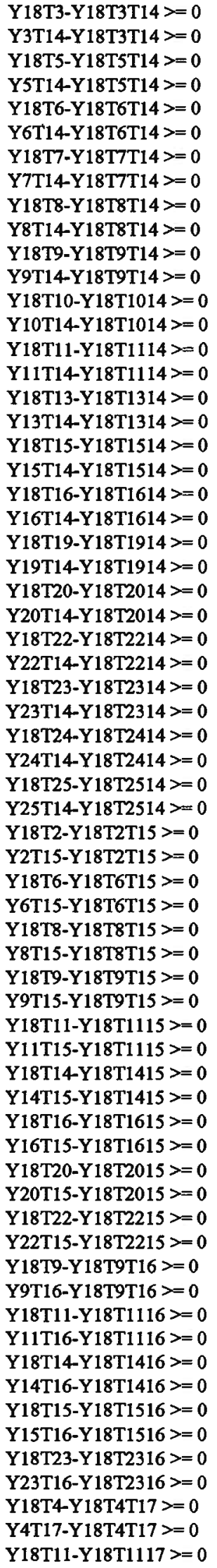

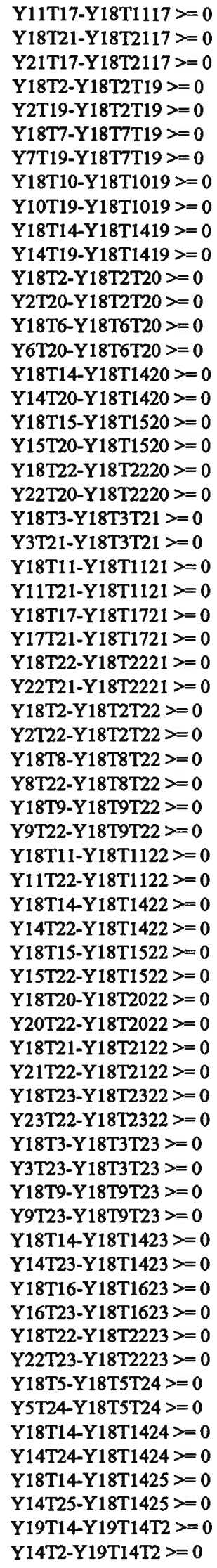


APPENDIX B : PROBLEM FORMULATION FILE EFFICIENT AIRLINE NETWORK

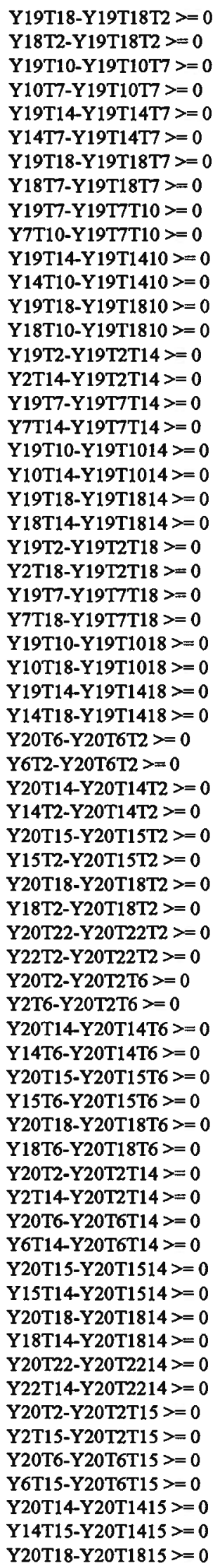

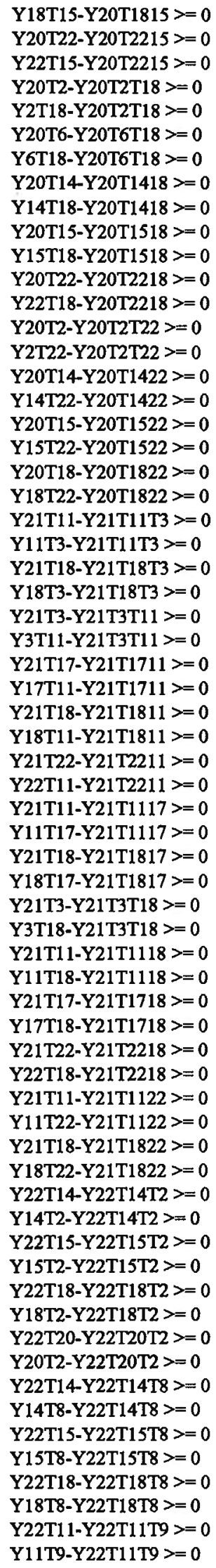


APPENDIX B : PROBLEM FORMULATION FILE EFFICIENT AIRLINE NETWORK

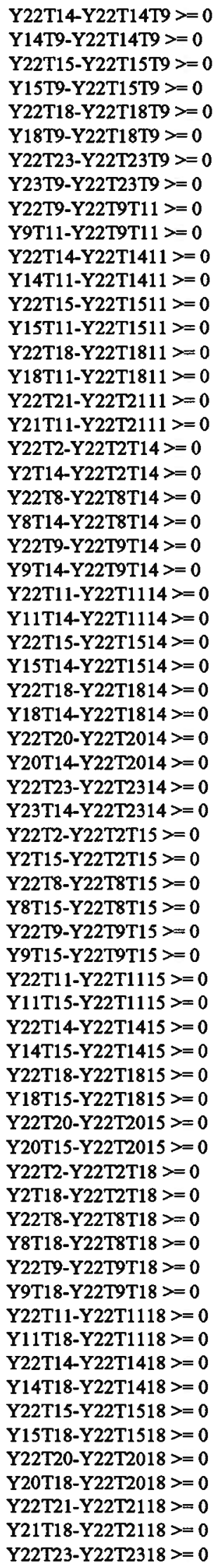

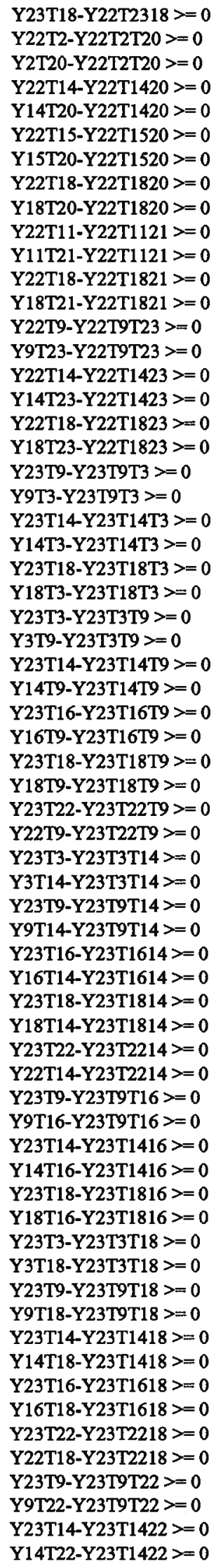




\section{APPENDIX B : PROBLEM FORMULATION FILE EFFICIENT AIRLINE NETWORK}

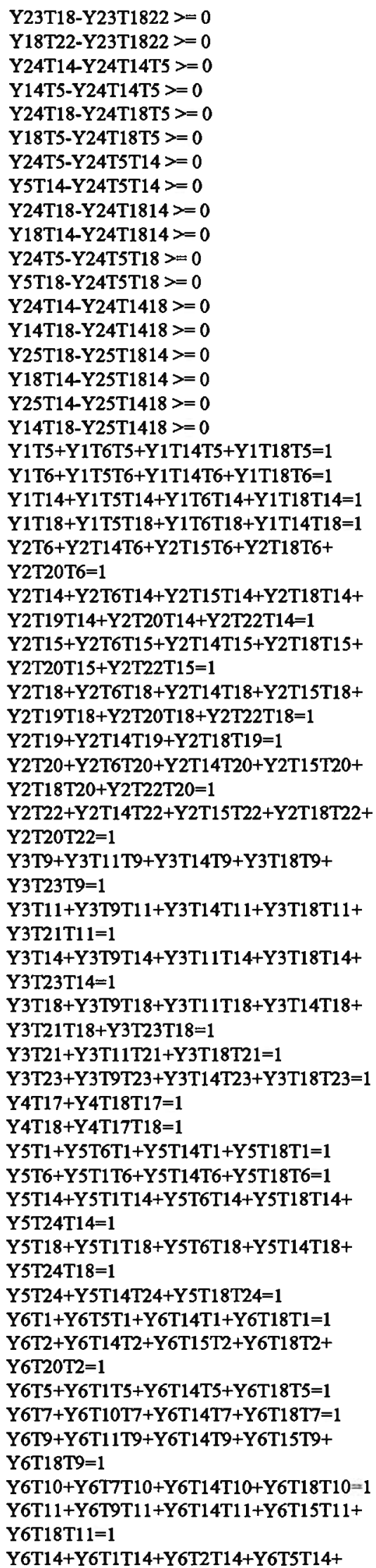

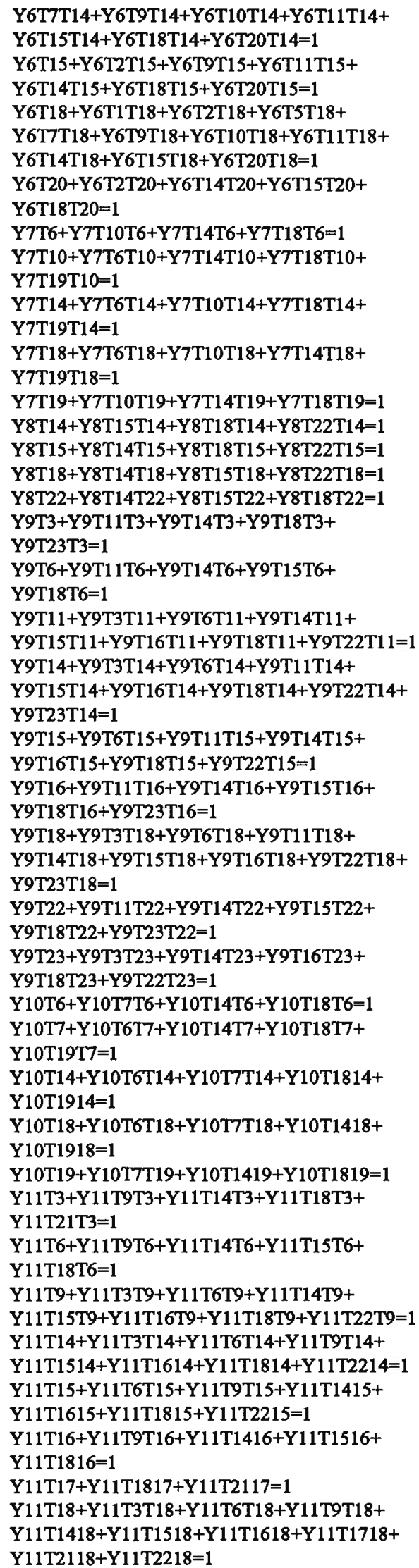




\section{APPENDIX B : PROBLEM FORMULATION FILE EFFICIENT AIRLINE NETWORK}

$\mathrm{Y} 11 \mathrm{~T} 21+\mathrm{Y} 11 \mathrm{~T} 3 \mathrm{~T} 21+\mathrm{Y} 11 \mathrm{~T} 1721+\mathrm{Y} 11 \mathrm{~T} 1821+$

$Y 11 T 2221=1$

$\mathrm{Y} 11 \mathrm{~T} 22+\mathrm{Y} 11 \mathrm{~T} 9 \mathrm{~T} 22+\mathrm{Y} 11 \mathrm{~T} 1422+\mathrm{Y} 11 \mathrm{~T} 1522+$

$\mathrm{Y} 11 \mathrm{~T} 1822+\mathrm{Y} 11 \mathrm{~T} 2122=1$

$\mathrm{Y} 12 \mathrm{~T} 18=1$

$\mathrm{Y} 13 \mathrm{~T} 14+\mathrm{Y} 13 \mathrm{~T} 1814=1$

$\mathrm{Y} 13 \mathrm{~T} 18+\mathrm{Y} 13 \mathrm{~T} 1418=1$

$\mathrm{Y} 14 \mathrm{~T} 1+\mathrm{Y} 14 \mathrm{~T} 5 \mathrm{~T} 1+\mathrm{Y} 14 \mathrm{~T} 6 \mathrm{~T} 1+\mathrm{Y} 14 \mathrm{~T} 18 \mathrm{~T} 1=1$

$\mathrm{Y} 14 \mathrm{~T} 2+\mathrm{Y} 14 \mathrm{~T} 6 \mathrm{~T} 2+\mathrm{Y} 14 \mathrm{~T} 15 \mathrm{~T} 2+\mathrm{Y} 14 \mathrm{~T} 18 \mathrm{~T} 2+$

$\mathrm{Y} 14 \mathrm{~T} 19 \mathrm{~T} 2+\mathrm{Y} 14 \mathrm{~T} 20 \mathrm{~T} 2+\mathrm{Y} 14 \mathrm{~T} 22 \mathrm{~T} 2=1$

$\mathrm{Y} 14 \mathrm{~T} 3+\mathrm{Y} 14 \mathrm{~T} 9 \mathrm{~T} 3+\mathrm{Y} 14 \mathrm{~T} 11 \mathrm{~T} 3+\mathrm{Y} 14 \mathrm{~T} 18 \mathrm{~T} 3+$

$\mathrm{Y} 14 \mathrm{~T} 23 \mathrm{~T} 3=1$

Y14T5+Y14T1T5+Y14T6T5+Y14T18T5+

$\mathrm{Y} 14 \mathrm{~T} 24 \mathrm{~T} 5=1$

Y14T6+Y14T1T6+Y14T2T6+Y14T5T6+

$\mathrm{Y} 14 \mathrm{~T} 7 \mathrm{~T} 6+\mathrm{Y} 14 \mathrm{~T} 9 \mathrm{~T} 6+\mathrm{Y} 14 \mathrm{~T} 10 \mathrm{~T} 6+\mathrm{Y} 14 \mathrm{~T} 11 \mathrm{~T} 6+$

$Y 14 T 15 \mathrm{~T} 6+Y 14 \mathrm{~T} 18 \mathrm{~T} 6+\mathrm{Y} 14 \mathrm{~T} 20 \mathrm{~T} 6=1$

$\mathrm{Y} 14 \mathrm{~T} 7+\mathrm{Y} 14 \mathrm{~T} 6 \mathrm{~T} 7+\mathrm{Y} 14 \mathrm{~T} 10 \mathrm{~T} 7+\mathrm{Y} 14 \mathrm{~T} 18 \mathrm{~T} 7+$

$\mathrm{Y} 14 \mathrm{~T} 19 \mathrm{~T} 7=1$

$\mathrm{Y} 14 \mathrm{~T} 8+\mathrm{Y} 14 \mathrm{~T} 15 \mathrm{~T} 8+\mathrm{Y} 14 \mathrm{~T} 18 \mathrm{~T} 8+\mathrm{Y} 14 \mathrm{~T} 2 \mathrm{~T} 8=1$

Y14T9+Y14T3T9+Y14T6T9+Y14T11T9+

Y14T15T9+Y14T16T9+Y14T18T9+Y14T22T9+

$Y 14 T 23 T 9=1$

$\mathrm{Y} 14 \mathrm{~T} 10+\mathrm{Y} 14 \mathrm{~T} 6 \mathrm{~T} 10+\mathrm{Y} 14 \mathrm{~T} 7 \mathrm{~T} 10+\mathrm{Y} 14 \mathrm{~T} 1810+$

Y14T1910=1

$\mathrm{Y} 14 \mathrm{~T} 11+\mathrm{Y} 14 \mathrm{~T} 3 \mathrm{~T} 11+\mathrm{Y} 14 \mathrm{~T} 6 \mathrm{~T} 11+\mathrm{Y} 14 \mathrm{~T} 9 \mathrm{~T} 11+$

$\mathrm{Y} 14 \mathrm{~T} 1511+\mathrm{Y} 14 \mathrm{~T} 1611+\mathrm{Y} 14 \mathrm{~T} 1811+\mathrm{Y} 14 \mathrm{~T} 2211=1$

Y14T13+Y14T1813=1

Y14T15+Y14T2T15+Y14T6T15+Y14T8T15+

Y14T9T15+Y14T1115+Y14T1615+Y14T1815+

$Y 14 T 2015+Y 14 T 2215=1$

Y14T16+Y14T9T16+Y14T1116+Y14T1516+

$\mathrm{Y} 14 \mathrm{~T} 1816+\mathrm{Y} 14 \mathrm{~T} 2316=1$

$\mathrm{Y} 14 \mathrm{~T} 18+\mathrm{Y} 14 \mathrm{~T} 1 \mathrm{~T} 18+\mathrm{Y} 14 \mathrm{~T} 2 \mathrm{~T} 18+\mathrm{Y} 14 \mathrm{~T} 3 \mathrm{~T} 18+$

$\mathrm{Y} 14 \mathrm{~T} 5 \mathrm{~T} 18+\mathrm{Y} 14 \mathrm{~T} 6 \mathrm{~T} 18+\mathrm{Y} 14 \mathrm{~T} 7 \mathrm{~T} 18+\mathrm{Y} 14 \mathrm{~T} 8 \mathrm{~T} 18+$

Y14T9T18+Y14T1018+Y14T1118+Y14T1318+

Y14T1518+Y14T1618+Y14T1918+Y14T2018+

$\mathrm{Y} 14 \mathrm{~T} 2218+\mathrm{Y} 14 \mathrm{~T} 2318+\mathrm{Y} 14 \mathrm{~T} 2418+\mathrm{Y} 14 \mathrm{~T} 2518=1$

Y14T19+Y14T2T19+Y14T7T19+Y14T1019+

$\mathrm{Y} 14 \mathrm{~T} 1819=1$

Y14T20+Y14T2T20+Y14T6T20+Y14T1520+

$\mathrm{Y} 14 \mathrm{~T} 1820+\mathrm{Y} 14 \mathrm{~T} 2220=1$

$\mathrm{Y} 14 \mathrm{~T} 22+\mathrm{Y} 14 \mathrm{~T} 2 \mathrm{~T} 22+\mathrm{Y} 14 \mathrm{~T} 8 \mathrm{~T} 22+\mathrm{Y} 14 \mathrm{~T} 9 \mathrm{~T} 22+$

Y14T1122+Y14T1522+Y14T1822+Y14T2022+

$\mathrm{Y} 14 \mathrm{~T} 2322=1$

Y14T23+Y14T3T23+Y14T9T23+Y14T1623+

$\mathrm{Y} 14 \mathrm{~T} 1823+\mathrm{Y} 14 \mathrm{~T} 2223=1$

Y14T24+Y14T5T24+Y14T1824=1

$\mathrm{Y} 14 \mathrm{~T} 25+\mathrm{Y} 14 \mathrm{~T} 1825=1$

$\mathrm{Y} 15 \mathrm{~T} 2+\mathrm{Y} 15 \mathrm{~T} 6 \mathrm{~T} 2+\mathrm{Y} 15 \mathrm{~T} 14 \mathrm{~T} 2+\mathrm{Y} 15 \mathrm{~T} 18 \mathrm{~T} 2+$

$\mathrm{Y} 15 \mathrm{~T} 20 \mathrm{~T} 2+\mathrm{Y} 15 \mathrm{~T} 22 \mathrm{~T} 2=1$

Y15T6+Y15T2T6+Y15T9T6+Y15T11T6+

Y15T14T6+Y15T18T6+Y15T20T6=1

$\mathrm{Y} 15 \mathrm{~T} 8+\mathrm{Y} 15 \mathrm{~T} 14 \mathrm{~T} 8+\mathrm{Y} 15 \mathrm{~T} 18 \mathrm{~T} 8+\mathrm{Y} 15 \mathrm{~T} 2 \mathrm{~T} 8=1$

$\mathrm{Y} 15 \mathrm{~T} 9+\mathrm{Y} 15 \mathrm{~T} 6 \mathrm{~T} 9+\mathrm{Y} 15 \mathrm{~T} 11 \mathrm{~T} 9+\mathrm{Y} 15 \mathrm{~T} 14 \mathrm{~T} 9+$

$\mathrm{Y} 15 \mathrm{~T} 16 \mathrm{~T} 9+\mathrm{Y} 15 \mathrm{~T} 18 \mathrm{~T} 9+\mathrm{Y} 15 \mathrm{~T} 22 \mathrm{~T} 9=1$

$\mathrm{Y} 15 \mathrm{~T} 11+\mathrm{Y} 15 \mathrm{~T} 6 \mathrm{~T} 11+\mathrm{Y} 15 \mathrm{~T} 9 \mathrm{~T} 11+\mathrm{Y} 15 \mathrm{~T} 1411+$

$\mathrm{Y} 15 \mathrm{~T} 1611+\mathrm{Y} 15 \mathrm{~T} 1811+\mathrm{Y} 15 \mathrm{~T} 2211=1$

Y15T14+Y15T2T14+Y15T6T14+Y15T8T14+ Y15T9T14+Y15T1114+Y15T1614+Y15T1814+ Y15T2014+Y15T2214=1

Y15T16+Y15T9T16+Y15T1116+Y15T1416+

$Y 15 T 1816=1$

$\mathrm{Y} 15 \mathrm{~T} 18+\mathrm{Y} 15 \mathrm{~T} 2 \mathrm{~T} 18+\mathrm{Y} 15 \mathrm{~T} 6 \mathrm{~T} 18+\mathrm{Y} 15 \mathrm{~T} 8 \mathrm{~T} 18+$

Y15T9T18+Y15T1118+Y15T1418+Y15T1618+
$\mathrm{Y} 15 \mathrm{~T} 2018+\mathrm{Y} 15 \mathrm{~T} 2218=1$

$\mathrm{Y} 15 \mathrm{~T} 20+\mathrm{Y} 15 \mathrm{~T} 2 \mathrm{~T} 20+\mathrm{Y} 15 \mathrm{~T} 6 \mathrm{~T} 20+\mathrm{Y} 15 \mathrm{~T} 1420+$

$\mathrm{Y} 15 \mathrm{~T} 1820+\mathrm{Y} 15 \mathrm{~T} 2220=1$

Y15T22+Y15T2T22+Y15T8T22+Y15T9T22+

$Y 15 T 1122+Y 15 T 1422+Y 15 T 1822+Y 15 T 2022=1$

$\mathrm{Y} 16 \mathrm{~T} 9+\mathrm{Y} 16 \mathrm{~T} 11 \mathrm{~T} 9+\mathrm{Y} 16 \mathrm{~T} 14 \mathrm{~T} 9+\mathrm{Y} 16 \mathrm{~T} 15 \mathrm{~T} 9+$

$\mathrm{Y} 16 \mathrm{~T} 18 \mathrm{~T} 9+\mathrm{Y} 16 \mathrm{~T} 23 \mathrm{~T} 9=1$

Y16T11+Y16T9T11+Y16T1411+Y16T1511+

$\mathrm{Y} 16 \mathrm{~T} 1811=1$

Y16T14+Y16T9T14+Y16T1114+Y16T1514+

$\mathrm{Y} 16 \mathrm{~T} 1814+\mathrm{Y} 16 \mathrm{~T} 2314=1$

Y16T15+Y16T9T15+Y16T1115+Y16T1415+

Y16T1815=1

Y16T18+Y16T9T18+Y16T1118+Y 16T1418+

$\mathrm{Y} 16 \mathrm{~T} 1518+\mathrm{Y} 16 \mathrm{~T} 2318=1$

$\mathrm{Y} 16 \mathrm{~T} 23+\mathrm{Y} 16 \mathrm{~T} 9 \mathrm{~T} 23+\mathrm{Y} 16 \mathrm{~T} 1423+\mathrm{Y} 16 \mathrm{~T} 1823=1$

$\mathrm{Y} 17 \mathrm{~T} 4+\mathrm{Y} 17 \mathrm{~T} 18 \mathrm{~T} 4=1$

$Y 17 T 11+Y 17 T 1811+Y 17 T 2111=1$

$\mathrm{Y} 17 \mathrm{~T} 18+\mathrm{Y} 17 \mathrm{~T} 4 \mathrm{~T} 18+\mathrm{Y} 17 \mathrm{~T} 1118+\mathrm{Y} 17 \mathrm{~T} 2118=1$

$\mathrm{Y} 17 \mathrm{~T} 21+\mathrm{Y} 17 \mathrm{~T} 1121+\mathrm{Y} 17 \mathrm{~T} 1821=1$

$\mathrm{Y} 18 \mathrm{~T} 1+\mathrm{Y} 18 \mathrm{~T} 5 \mathrm{~T} 1+\mathrm{Y} 18 \mathrm{~T} 6 \mathrm{~T} 1+\mathrm{Y} 18 \mathrm{~T} 14 \mathrm{Tl}=1$

$\mathrm{Y} 18 \mathrm{~T} 2+\mathrm{Y} 18 \mathrm{~T} 6 \mathrm{~T} 2+\mathrm{Y} 18 \mathrm{~T} 14 \mathrm{~T} 2+\mathrm{Y} 18 \mathrm{~T} 15 \mathrm{~T} 2+$

$\mathrm{Y} 18 \mathrm{~T} 19 \mathrm{~T} 2+\mathrm{Y} 18 \mathrm{~T} 20 \mathrm{~T} 2+\mathrm{Y} 18 \mathrm{~T} 22 \mathrm{~T} 2=1$

$\mathrm{Y} 18 \mathrm{~T} 3+\mathrm{Y} 18 \mathrm{~T} 9 \mathrm{~T} 3+\mathrm{Y} 18 \mathrm{~T} 11 \mathrm{~T} 3+\mathrm{Y} 18 \mathrm{~T} 14 \mathrm{~T} 3+$

$\mathrm{Y} 18 \mathrm{~T} 21 \mathrm{~T} 3+\mathrm{Y} 18 \mathrm{~T} 23 \mathrm{~T} 3=1$

$\mathrm{Y} 18 \mathrm{~T} 4+\mathrm{Y} 18 \mathrm{~T} 17 \mathrm{~T} 4=1$

Y18T5+Y18T1T5+Y18T6T5+Y18T14T5+

$\mathrm{Y} 18 \mathrm{~T} 24 \mathrm{~T} 5=1$

Y18T6+Y18T1T6+Y18T2T6+Y18T5T6+

$\mathrm{Y} 18 \mathrm{TTT} 6+\mathrm{Y} 18 \mathrm{~T} 9 \mathrm{~T} 6+\mathrm{Y} 18 \mathrm{~T} 10 \mathrm{~T} 6+\mathrm{Y} 18 \mathrm{~T} 11 \mathrm{~T} 6+$

$\mathrm{Y} 18 \mathrm{~T} 14 \mathrm{~T} 6+\mathrm{Y} 18 \mathrm{~T} 15 \mathrm{~T} 6+\mathrm{Y} 18 \mathrm{~T} 20 \mathrm{~T} 6=1$

$\mathrm{Y} 18 \mathrm{~T} 7+\mathrm{Y} 18 \mathrm{~T} 6 \mathrm{~T} 7+\mathrm{Y} 18 \mathrm{~T} 10 \mathrm{~T} 7+\mathrm{Y} 18 \mathrm{~T} 14 \mathrm{~T} 7+$

$\mathrm{Y} 18 \mathrm{~T} 19 \mathrm{~T} 7=1$

$\mathrm{Y} 18 \mathrm{~T} 8+\mathrm{Y} 18 \mathrm{~T} 14 \mathrm{~T} 8+\mathrm{Y} 18 \mathrm{~T} 15 \mathrm{~T} 8+\mathrm{Y} 18 \mathrm{~T} 22 \mathrm{~T} 8=1$

$\mathrm{Y} 18 \mathrm{~T} 9+\mathrm{Y} 18 \mathrm{~T} 3 \mathrm{~T} 9+\mathrm{Y} 18 \mathrm{~T} 6 \mathrm{~T} 9+\mathrm{Y} 18 \mathrm{~T} 11 \mathrm{~T} 9+$

Y18T14T9+Y18T15T9+Y18T16T9+Y18T22T9+

$\mathrm{Y} 18 \mathrm{~T} 23 \mathrm{~T} 9=1$

$\mathrm{Y} 18 \mathrm{~T} 10+\mathrm{Y} 18 \mathrm{~T} 6 \mathrm{~T} 10+\mathrm{Y} 18 \mathrm{~T} 7 \mathrm{~T} 10+\mathrm{Y} 18 \mathrm{~T} 1410+$

$\mathrm{Y} 18 \mathrm{~T} 1910=1$

$\mathrm{Y} 18 \mathrm{~T} 11+\mathrm{Y} 18 \mathrm{~T} 3 \mathrm{~T} 11+\mathrm{Y} 18 \mathrm{~T} 6 \mathrm{~T} 11+\mathrm{Y} 18 \mathrm{~T} 9 \mathrm{~T} 11+$

$\mathrm{Y} 18 \mathrm{~T} 1411+\mathrm{Y} 18 \mathrm{~T} 1511+\mathrm{Y} 18 \mathrm{~T} 1611+\mathrm{Y} 18 \mathrm{~T} 1711+$

$Y 18 T 2111+Y 18 T 2211=1$

$Y 18 \mathrm{~T} 12=1$

$Y 18 \mathrm{~T} 13+Y 18 \mathrm{~T} 1413=1$

Y18T14+Y18T1T14+Y18T2T14+Y 18T3T14+ Y18T5T14+Y18T6T14+Y18T7T14+Y18T8T14+ Y18T9T14+Y 18T1014+Y18T1114+Y18T1314+ Y18T1514+Y18T1614+Y18T1914+Y18T2014+ $\mathrm{Y} 18 \mathrm{~T} 2214+\mathrm{Y} 18 \mathrm{~T} 2314+\mathrm{Y} 18 \mathrm{~T} 2414+\mathrm{Y} 18 \mathrm{~T} 2514=1$ $\mathrm{Y} 18 \mathrm{~T} 15+\mathrm{Y} 18 \mathrm{~T} 2 \mathrm{~T} 15+\mathrm{Y} 18 \mathrm{~T} 6 \mathrm{~T} 15+\mathrm{Y} 18 \mathrm{~T}$ TT15+ Y18T9T15+Y18T1115+Y18T1415+Y18T1615+ $\mathrm{Y} 18 \mathrm{~T} 2015+\mathrm{Y} 18 \mathrm{~T} 2215=1$

Y18T16+Y18T9T16+Y18T1116+Y18T1416+

$\mathrm{Y} 18 \mathrm{~T} 1516+\mathrm{Y} 18 \mathrm{~T} 2316=1$

$\mathrm{Y} 18 \mathrm{~T} 17+\mathrm{Y} 18 \mathrm{~T} 4 \mathrm{~T} 17+\mathrm{Y} 18 \mathrm{~T} 1117+\mathrm{Y} 18 \mathrm{~T} 2117=1$ Y18T19+Y 18T2T19+Y 18T7T19+Y18T1019+ $\mathrm{Y} 18 \mathrm{~T} 1419=1$ $\mathrm{Y} 18 \mathrm{~T} 20+\mathrm{Y} 18 \mathrm{~T} 2 \mathrm{~T} 20+\mathrm{Y} 18 \mathrm{~T} 6 \mathrm{~T} 20+\mathrm{Y} 18 \mathrm{~T} 1420+$ $\mathrm{Y} 18 \mathrm{~T} 1520+\mathrm{Y} 18 \mathrm{~T} 2220=1$

Y18T21+Y18T3T21+Y18T1121+Y18T1721+ $\mathrm{Y} 18 \mathrm{~T} 2221=1$

Y18T22+Y18T2T22+Y18T8T22+Y18T9T22+ Y18T1122+Y18T1422+Y18T1522+Y18T2022+ $Y 18 T 2122+Y 18 T 2322=1$

Y18T23+Y18T3T23+Y18T9T23+Y18T1423+ 


\section{APPENDIX B : PROBLEM FORMULATION FILE EFFICIENT AIRLINE NETWORK}

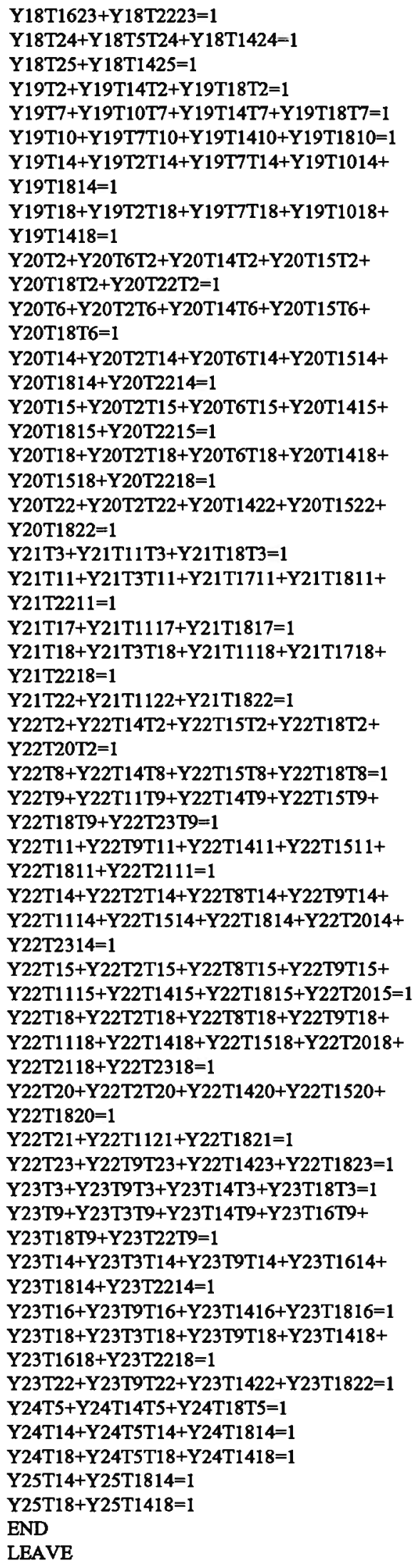




\section{APPENDIX B: PROBLEM FORMULATION FILE EFFICIENT PASSENGERS NETWORK}

MTN

+709.72Y1T5+844.33Y1T6T5+433.95Y1T14T5+ 288.26Y1T18T5+747.20Y1T6+806.84Y1T5T6+ 462.92Y1T14T6+235.72Y1T18T6+370.34Y1T14+ 255.03Y1T5T14+278.89Y1T6T14+238.56Y1T18T14+ 211.15Y1T18+179.77Y1T5T18+207.32Y1T6T18+ 237.99Y1T14T18+291.01Y2T6+159.32Y2T14T6+ 250.77Y2T15T6+142.85Y2T18T6+293.66Y2T20T6+ 74.73Y2T14+201.36Y2T6T14+124.11Y2T15T14+ 145.69Y2T18T14+134.90Y2T19T14+130.07Y2T20T14+ $177.22 \mathrm{Y} 2 \mathrm{~T} 22 \mathrm{~T} 14+738.68 \mathrm{Y} 2 \mathrm{~T} 15+380.56 \mathrm{Y} 2 \mathrm{~T} 6 \mathrm{~T} 15+$ $140.86 \mathrm{Y} 2 \mathrm{~T} 14 \mathrm{~T} 15+184.32 \mathrm{Y} 2 \mathrm{~T} 18 \mathrm{~T} 15+791.22 \mathrm{Y} 2 \mathrm{~T} 20 \mathrm{~T} 15+$ $830.70 \mathrm{Y} 2 \mathrm{~T} 22 \mathrm{~T} 15+90.12 \mathrm{Y} 2 \mathrm{~T} 18+172.39 \mathrm{Y} 2 \mathrm{~T} 6 \mathrm{~T} 18+$ 133.20Y2T14T18+140.86Y2T15T18+127.52Y2T19T18+ 153.36Y2T20T18+183.75Y2T22T18+723.92Y2T19+ 351.02Y2T14T19+469.74Y2T18T19+374.60Y2T20+ $326.03 \mathrm{Y} 2 \mathrm{~T} 6 \mathrm{~T} 20+432.82 \mathrm{Y} 2 \mathrm{~T} 14 \mathrm{~T} 20+338.81 \mathrm{Y} 2 \mathrm{~T} 15 \mathrm{~T} 20+$ 198.80 $2 \mathrm{~T} 18 \mathrm{~T} 20+493.31 \mathrm{Y} 2 \mathrm{~T} 22 \mathrm{~T} 20+758.85 \mathrm{Y} 2 \mathrm{~T} 22+$ 202.21Y2T14T22+810.54Y2T15T22+235.44Y2T18T22+ $811.96 \mathrm{Y} 2 \mathrm{~T} 20 \mathrm{~T} 22+726.47 \mathrm{Y} 3 \mathrm{~T} 9+781.28 \mathrm{Y} 3 \mathrm{~T} 11 \mathrm{~T} 9+$ 469.17Y3T14T9+225.50Y3T18T9+802.87Y3T23T9+ 718.52Y3T11+789.24Y3T9T11+353.86Y3T14T11+ 219.82Y3T18T11+789.52Y3T21T11+395.61Y3T14+ 452.13Y3T9T14+328.87Y3T11T14+217.83Y3T18T14+ 462.07Y3T23T14+160.84Y3T18+211.30Y3T9T18+ 197.66Y3T11T18+220.67Y3T14T18+232.31Y3T21T18+ 232.31Y3T23T18+714.83Y3T21+793.21Y3T11T21+ 485.36Y3T18T21+717.95Y3T23+811.39Y3T9T23+ 487.63Y3T14T23+311.83Y3T18T23+719.66Y4T17+ 449.57Y4T18T17+200.08Y4T18+276.90Y4T17T18+ 709.72Y5T1+844.33Y5T6T1+433.95Y5T14T1+ 288.26Y5T18T1+750.33Y5T6+803.72Y5T1T6+ 255.60Y5T14T6+208.17Y5T18T6+202.07Y5T14+ 249.35Y5T1T14+282.01Y5T6T14+182.61Y5T18T14+ 267.81Y5T24T14+137.46Y5T18+193.12Y5T1T18+ 224.64Y5T6T18+172.67Y5T14T18+191.42Y5T24T18+ 713.12Y5T24+804.00Y5T14T24+339.95Y5T18T24+ 747.20Y6T1+806.84Y6T5T1+349.32Y6T14T1+ $235.72 \mathrm{Y} 6 \mathrm{~T} 18 \mathrm{~T} 1+291.01 \mathrm{Y} 6 \mathrm{~T} 2+159.32 \mathrm{Y} 6 \mathrm{~T} 14 \mathrm{~T} 2+$ $264.97 \mathrm{Y} 6 \mathrm{~T} 15 \mathrm{~T} 2+142.85 \mathrm{Y} 6 \mathrm{~T} 18 \mathrm{~T} 2+251.06 \mathrm{Y} 6 \mathrm{~T} 20 \mathrm{~T} 2+$ 750.33Y6T5+803.72Y6T1T5+255.60Y6T14T5+ 208.17Y6T18T5+753.17Y6T7+465.48Y6T10T7+ 236.86Y6T14T7+185.17Y6T18T7+383.40Y6T9+ 450.71Y6T11 T9+251.34Y6T14T9+821.90Y6T15T9+ $180.34 \mathrm{Y} 6 \mathrm{~T} 18 \mathrm{~T} 9+408.68 \mathrm{Y} 6 \mathrm{~T} 10+348.47 \mathrm{Y} 6 \mathrm{~T} 7 \mathrm{~T} 10+$ $161.88 \mathrm{Y} 6 \mathrm{~T} 14 \mathrm{~T} 10+140.30 \mathrm{Y} 6 \mathrm{~T} 18 \mathrm{~T} 10+742.94 \mathrm{Y} 6 \mathrm{~T} 11+$ 794.06Y6T9T11+363.24Y6T14T11+814.23Y6T15T11+ 174.66Y6T18T11+98.01Y6T14+144.84Y6T1T14+ 161.31Y6T2T14+139.44Y6T5T14+151.94Y6T7T14+ 130.36Y6T9T14+151.37Y6T10T14+140.30Y6T11T14+ 132.91Y6T15T14+130.07Y6T18T14+143.14Y6T20T14+ 399.59Y6T15+314.96Y6T2T15+450.71Y6T9T15+ 341.08Y6T11T15+152.51Y6T14T15+168.70Y6T18T15+ $293.09 \mathrm{Y} 6 \mathrm{~T} 20 \mathrm{~T} 15+51.31 \mathrm{Y} 6 \mathrm{~T} 18+145.41 \mathrm{Y} 6 \mathrm{~T} 1 \mathrm{~T} 18+$ 159.61Y6T2T18+135.18Y6T5T18+143.42Y6T7T18+ $116.72 \mathrm{Y} 6 \mathrm{~T} 9 \mathrm{~T} 18+144.56 \mathrm{Y} 6 \mathrm{~T} 10 \mathrm{~T} 18+122.69 \mathrm{Y} 6 \mathrm{~T} 11 \mathrm{~T} 18+$ 159.04Y6T14T18+149.67Y6T15T18+152.22Y6T20T18+ 227.91 Y $6 \mathrm{~T} 20+355.57 \mathrm{Y} 6 \mathrm{~T} 2 \mathrm{~T} 20+345.06 \mathrm{Y} 6 \mathrm{~T} 14 \mathrm{~T} 20+$ 290.82Y6T15T20+168.98Y6T18T20+753.17Y7T6+ 465.48Y7T10T6+236.86Y7T14T6+199.37Y7T18T6+ 717.10Y7T10+501.54Y7T6T10+227.77Y7T14T10+ 212.43Y7T18T10+784.12Y7T19T10+153.74Y7T14+ 256.45Y7T6T14+211.30Y7T10T14+173.81Y7T18T14+ 212.43Y7T19T14+142.85Y7T18+213.28Y7T6T18+ 190.28Y7T10T18+168.13Y7T14T18+190.85Y7T19T18+
716.25Y7T19+784.98Y7T10T19+471.16Y7T14T19+ 469.45Y7T18T19+378.57Y8T14+433.10Y8T15T14+ 482.23Y8T18T14+445.31Y8T22T14+721.08Y8T15+ 438.50Y8T14T15+833.26Y8T18T15+786.40Y8T22T15+ 749.48Y8 $18+459.23 \mathrm{Y} 8 \mathrm{~T} 14 \mathrm{~T} 18+804.86 \mathrm{Y} 8 \mathrm{~T} 15 \mathrm{~T} 18+$ 806.84Y8T22T18+714.54Y8T22+457.24Y8T14T22+ 792.93Y8T15T22+841.78Y8T18T22+726.47Y9T3+ 781.28Y9T11T3+469.17Y9T14T3+225.50Y9T18T3+ 802.87Y9T23T3+383.40Y9T6+450.71Y9T11T6+ 251.34Y9T14T6+821.90Y9T15T6+194.54Y9T18T6+ 715.96Y9T11+791.79Y9T3T11+821.04Y9T6T11+ 344.21Y9T14T11+794.35Y9T15T11+804.57Y9T16 T11+ 213.57Y9T18T11+805.71Y9T22T11+177.22Y9T14+ 177.78Y9T3T14+191.98Y9T6T14+184.32Y9T11T14+ 226.63Y9T15T14+194.54Y9T16T14+168.98Y9T18T14+ 179.20Y9T22T14+198.23Y9T23T14+727.61Y9T15+ 356.99Y9T6T15+782.70Y9T11T15+218.68Y9T14T15+ 806.28Y9T16T15+221.80Y9T18T15+449.29Y9T22T15+ 727.61Y9T16+792.93Y9T11T16+484.79Y9T14T16+ $806.28 \mathrm{Y} 9 \mathrm{~T} 15 \mathrm{~T} 16+240.55 \mathrm{Y} 9 \mathrm{~T} 18 \mathrm{~T} 16+805.14 \mathrm{Y} 9 \mathrm{~T} 23 \mathrm{~T} 16+$ 140.39Y9T18+174.94Y9T3T18+148.82Y9T6T18+ 152.51Y9T11T18+182.61Y9T14T18+243.39Y9T15T18+ 176.93Y9T16T18+185.74Y9T22T18+195.68Y9T23T18+ 732.44Y9T22+789.24Y9T11T22+251.62Y9T14T22+ 799.46Y9T15T22+230.32Y9T18T22+807.70Y9T23T $22+$ 738.12Y9T23+791.22Y9T3T23+477.97Y9T14T23+ 794.63Y9T16T23+291.38Y9T18T23+802.02Y9T22T23+ 292.71Y10T6+348.47Y10T7T6+161.88Y10T14T6+ 140.30Y10T18T6+717.10Y10T7+501.54Y10T6T7+ 213.57Y10T14T7+212.43Y10T18T7+784.12Y10T19T7+ 74.73Y10T14+188.86Y10T6T14+130.07Y10T7T14+ 143.14Y10T1814+146.26Y10T1914+79.84Y10T18+ 159.89 Y10T6T18+135.75Y10T7T18+135.75Y10T1418+ 138.88Y10T1918+721.08Y10T19+780.15Y10T7T19+ 353.58Y10T1419+467.18Y10T1819+718.52Y11T3+ 789.24Y11T9T3+353.86Y11T14T3+219.82Y11T18T3+ 789.52Y11T21T3+742.94Y11T6+794.06Y11T9T6+ 363.24Y11T14T6+814.23Y11T15T6+174.66Y11T18T6+ $715.96 \mathrm{Y} 11 \mathrm{~T} 9+791.79 \mathrm{Y} 11 \mathrm{~T} 3 \mathrm{~T} 9+821.04 \mathrm{Y} 11 \mathrm{~T} 6 \mathrm{~T} 9+$ 344.21Y11T14T9+794.35Y11T15T9+804.57Y11T16T9+ 199.37Y11T18T9+805.71Y11T22T9+268.29Y11T14+ 240.83Y11T3T14+317.23Y11T6T14+271.22Y11T9T14+ 318.36Y11T1514+310.70Y11T1614+177.50Y11T1814+ 457.24Y11T2214+719.94Y11T15+837.23Y11T6T15+ 790.37Y11T9T15+330.58Y11T1415+808.83Y11T1615+ 187.72Y11T1815+798.32Y11T2215+730.16Y11T16+ 790.37Y11T9T16+483.08Y11T1416+798.61Y11T1516+ 234.87Y11T1816+716.53Y11T17+342.79Y11T1817+ 805.42Y11T2117+110.26Y11T18+152.79Y11T3T18+ 160.46Y11T6T18+129.79Y11T9T18+180.91Y11T1418+ $178.92 \mathrm{Y} 11 \mathrm{~T} 1518+165.29 \mathrm{Y} 11 \mathrm{~T} 1618+145.98 \mathrm{Y} 11 \mathrm{~T} 1718+$ 174.38Y11T2118+236.57Y11T2218+727.89Y11T21+ 780.15Y11T3T21+794.06Y11T1721+828.43Y11T1821+ 797.19Y11T2221+726.47Y11T22+795.20Y11T9T22+ 349.32Y11T1422+791.79Y11T1522+224.64Y11T1822+ 798.61Y11T2122+373.74Y12T18+753.74Y13T14+ 281.44Y13T1814+255.22Y13T18+316.09Y13T1418+ $370.34 \mathrm{Y} 14 \mathrm{Tl}+212.43 \mathrm{Y} 14 \mathrm{~T} 5 \mathrm{Tl}+264.69 \mathrm{Y} 14 \mathrm{~T} 6 \mathrm{Tl}+$ 224.36Y14T18T1+74.73Y14T2+201.36Y14T6T2+ 124.11Y14T15T2+145.69Y $14 \mathrm{~T} 18 \mathrm{~T} 2+134.90 \mathrm{Y} 14 \mathrm{~T} 19 \mathrm{~T} 2+$ $130.07 \mathrm{Y} 14 \mathrm{~T} 20 \mathrm{~T} 2+177.22 \mathrm{Y} 14 \mathrm{~T} 22 \mathrm{~T} 2+395.61 \mathrm{Y} 14 \mathrm{~T} 3+$ 452.13Y14T9T3+328.87Y14T11T3+217.83Y14T18T3+ 462.07Y14T23T3+202.07Y $14 \mathrm{~T} 5+249.35 \mathrm{Y} 14 \mathrm{~T} 1 \mathrm{~T} 5+$ 282.01Y14T6T5+182.61Y14T18T5+225.21Y14T24T5+ 95.74Y14T6+144.84Y14T1T6+161.31Y14T2T6+ 


\section{APPENDIX B: PROBLEM FORMULATION FILE EFFICIENT PASSENGERS NETWORK}

139.44Y14T5T6+151.94Y14T7T6+130.36Y14T9T6+ 151.37Y14T10T6+140.30Y14T11T6+132.91Y14T15T6+ $130.07 \mathrm{Y} 14 \mathrm{~T} 18 \mathrm{~T} 6+143.14 \mathrm{Y} 14 \mathrm{~T} 20 \mathrm{~T} 6+153.74 \mathrm{Y} 14 \mathrm{~T} 7+$ $256.45 \mathrm{Y} 14 \mathrm{~T} 6 \mathrm{~T} 7+197.10 \mathrm{Y} 14 \mathrm{~T} 10 \mathrm{~T} 7+173.81 \mathrm{Y} 14 \mathrm{~T} 18 \mathrm{~T} 7+$ 212.43Y14T19T7+726.47Y14T8+433.10Y14T15T8+ 482.23Y14T18T8+445.31Y14T22T8+177.22Y14T9+ 177.78Y14T3T9+191.98Y14T6T9+184.32Y14T11T9+ 226.63Y14T15T9+194.54Y14T16 T9+168.98Y14T18T9+ $179.20 \mathrm{Y} 14 \mathrm{~T} 22 \mathrm{~T} 9+198.23 \mathrm{Y} 14 \mathrm{~T} 23 \mathrm{~T} 9+74.73 \mathrm{Y} 14 \mathrm{~T} 10+$ 188.86Y14T6T10+130.07Y14T7T10+143.14Y14T1810+ 146.26Y14T1910+268.29Y14T11+240.83Y14T3T11+ 317.23Y14T6 T11+271.22Y14T9T11+318.36Y14T1511+ $310.70 \mathrm{Y} 14 \mathrm{~T} 1611+177.50 \mathrm{Y} 14 \mathrm{~T} 1811+457.24 \mathrm{Y} 14 \mathrm{~T} 2211+$ 753.74Y $14 \mathrm{~T} 13+281.44 \mathrm{Y} 14 \mathrm{~T} 1813+65.35 \mathrm{Y} 14 \mathrm{~T} 15+$ $130.92 \mathrm{Y} 14 \mathrm{~T} 2 \mathrm{~T} 15+165.57 \mathrm{Y} 14 \mathrm{~T} 6 \mathrm{~T} 15+112.75 \mathrm{Y} 14 \mathrm{~T} 8 \mathrm{~T} 15+$ $140.86 \mathrm{Y} 14 \mathrm{~T} 9 \mathrm{~T} 15+131.49 \mathrm{Y} 14 \mathrm{~T} 1115+156.20 \mathrm{Y} 14 \mathrm{~T} 1615+$ 143.14Y14T1815+115.30Y14T2015+143.42Y14T2215+ 411.23Y14T16+453.26Y14T9T16+454.12Y14T1116+ 443.89Y14T1516+247.08Y14T1816+464.34Y14T2316+ $56.56 \mathrm{Y} 14 \mathrm{~T} 18+102.24 \mathrm{Y} 14 \mathrm{~T} 1 \mathrm{~T} 18+117.58 \mathrm{Y} 14 \mathrm{~T} 2 \mathrm{~T} 18+$ 135.18Y14T3T18+108.77Y14T5T18+127.80Y14T6T18+ 123.82Y14T7T18+126.95Y14T8T18+105.08Y14T9T18+ $117.58 \mathrm{Y} 14 \mathrm{~T} 1018+111.90 \mathrm{Y} 14 \mathrm{~T} 1118+123.82 \mathrm{Y} 14 \mathrm{~T} 1318+$ $108.20 \mathrm{Y} 14 \mathrm{~T} 1518+151.66 \mathrm{Y} 14 \mathrm{~T} 1618+135.18 \mathrm{Y} 14 \mathrm{~T} 1918+$ 102.24Y14T2018+135.47Y14T2218+153.08Y14T2318+ 112.18Y14T2418+109.91Y14T2518+397.88Y14T19+ 329.16Y14T2T19+441.62Y14T7T19+328.87Y14T1019+ $357.56 \mathrm{Y} 14 \mathrm{~T} 1819+713.98 \mathrm{Y} 14 \mathrm{~T} 20+441.34 \mathrm{Y} 14 \mathrm{~T} 2 \mathrm{~T} 20+$ 281.44Y14T6T20+433.95Y14T1520+200.22Y14T1820+ 828.43Y14T2220+130.14Y14T22+165.29Y14T2T22+ $120.42 \mathrm{Y} 14 \mathrm{~T} 8 \mathrm{~T} 22+159.89 \mathrm{Y} 14 \mathrm{~T} 9 \mathrm{~T} 22+194.82 \mathrm{Y} 14 \mathrm{~T} 1122+$ 153.08Y14T1522+180.06Y14T1822+192.84Y14T2022+ 154.50Y14T2322+752.32Y14T23+453.26Y14T3T23+ 463.77Y14T9T23+471.16Y14T1623+312.12Y14T1823+ 453.55Y14T2223+733.29Y14T24+783.84Y14T5T24+ $347.05 \mathrm{Y} 14 \mathrm{~T} 1824+71.57 \mathrm{Y} 14 \mathrm{~T} 25+175.80 \mathrm{Y} 14 \mathrm{~T} 1825+$ 738.68Y15T2+380.56Y15T6T2+140.86Y15T14T2+ 184.32Y15T18T2+436.22Y15T20T2+830.70Y15T22T2+ $399.59 \mathrm{Y} 15 \mathrm{~T} 6+314.96 \mathrm{Y} 15 \mathrm{~T} 2 \mathrm{~T} 6+450.71 \mathrm{Y} 15 \mathrm{~T} 9 \mathrm{~T} 6+$ 341.08Y15T11T6+152.51Y15T14T6+168.70Y15T18T6+ $250.49 \mathrm{Y} 15 \mathrm{~T} 20 \mathrm{~T} 6+721.08 \mathrm{Y} 15 \mathrm{~T} 8+438.50 \mathrm{Y} 15 \mathrm{~T} 14 \mathrm{~T} 8+$ 833.26Y15T18T8+786.40Y15T22T8+727.61Y15T9+ $356.99 \mathrm{Y} 15 \mathrm{~T} 6 \mathrm{~T} 9+782.70 \mathrm{Y} 15 \mathrm{~T} 11 \mathrm{~T} 9+218.68 \mathrm{Y} 15 \mathrm{~T} 14 \mathrm{~T} 9+$ 806.28Y15T16T9+193.40Y15T18T9+449.29Y15T22T9+ 719.94Y15T11+837.23Y15T6T11+790.37Y15T9T11+ $330.58 \mathrm{Y} 15 \mathrm{~T} 1411+808.83 \mathrm{Y} 15 \mathrm{~T} 1611+187.72 \mathrm{Y} 15 \mathrm{~T} 1811+$ 798.32Y15T2211+65.35Y15T14+130.92Y15T2T14+ 165.57Y15T6T14+1 12.75Y15T8T14+140.86Y15T9T14+ 131.49Y15T1114+156.20Y15T1614+143.14Y15T1814+ 115.30Y15T2014+143.42Y15T2214+731.87Y15T16+ 802.02Y15T9T16+796.90Y15T1116+471.16Y15T1416+ 257.30Y15T1816+110.76Y15T18+157.62Y15T2T18+ $165.00 \mathrm{Y} 15 \mathrm{~T} 6 \mathrm{~T} 18+149.95 \mathrm{Y} 15 \mathrm{~T} 8 \mathrm{~T} 18+169.83 \mathrm{Y} 15 \mathrm{~T} 9 \mathrm{~T} 18+$ 156.48Y15T1118+126.38Y15T1418+152.79Y15T1618+ 138.59Y15T2018+178.35Y15T2218+721.93Y15T20+ 452.98Y15T2T20+375.45Y15T6T20+781.00Y15T1420+ 196.24Y15T1820+814.51Y15T2220+377.15Y15T22+ $375.73 \mathrm{Y} 15 \mathrm{~T} 2 \mathrm{~T} 22+313.82 \mathrm{Y} 15 \mathrm{~T} 8 \mathrm{~T} 22+451.84 \mathrm{Y} 15 \mathrm{~T} 9 \mathrm{~T} 22+$ 438.21Y15T1122+181.19Y15T1422+218.68Y15T1822+ 456.39Y15T2022+727.61Y16T9+792.93Y16T11 T9+ 484.79Y16 $14 \mathrm{~T} 9+806.28 \mathrm{Y} 16 \mathrm{~T} 15 \mathrm{~T} 9+240.55 \mathrm{Y} 16 \mathrm{~T} 18 \mathrm{~T} 9+$ 805.14Y16T23T9+730.16Y16T11+790.37Y16T9T11+ 483.08Y16T1411+798.61Y16T1511+234.87Y $16 \mathrm{~T} 1811+$ 411.23Y16 $14+453.26 \mathrm{Y} 16 \mathrm{~T} 9 \mathrm{~T} 14+454.12 \mathrm{Y} 16 \mathrm{~T} 1114+$ 443.89Y16T1514+247.08Y16T1814+464.34Y16T2314+
731.87Y16T15+802.02Y16T9T15+796.90Y16T1115+ 471.16Y16T1415+257.30Y16T1815+184.88Y16T18+ $226.63 \mathrm{Y} 16 \mathrm{~T} 9 \mathrm{~T} 18+223.51 \mathrm{Y} 16 \mathrm{~T} 1118+264.69 \mathrm{Y} 16 \mathrm{~T} 1418+$ $247.65 \mathrm{Y} 16 \mathrm{~T} 1518+248.78 \mathrm{Y} 16 \mathrm{~T} 2318+720.22 \mathrm{Y} 16 \mathrm{~T} 23+$ $812.52 \mathrm{Y} 16 \mathrm{~T} 9 \mathrm{~T} 23+503.25 \mathrm{Y} 16 \mathrm{~T} 1423+312.68 \mathrm{Y} 16 \mathrm{~T} 1823+$ 719.66Y17T4+449.57Y17T18T4+716.53Y17T11+ 456.39Y17T1811+805.42Y17T2111+387.94Y17T18+ 433.38Y17T4T18+437.08Y17T1118+475.42Y17T2118+ 730.73Y17T21+791.22Y17T1121+835.53Y17T1821+ 211.15Y18T1+179.77Y18T5T1+193.12Y18T6T1+ 237.99Y18T14T1+87.22Y18T2+172.39Y18T6T2+ 133.20Y18T14T2+140.86Y18T15T2+127.52Y18T19T2+ $139.16 \mathrm{Y} 18 \mathrm{~T} 20 \mathrm{~T} 2+183.75 \mathrm{Y} 18 \mathrm{~T} 22 \mathrm{~T} 2+160.84 \mathrm{Y} 18 \mathrm{~T} 3+$ 211.30Y18T9T3+197.66Y18T11T3+220.67Y18T14T3+ 232.31Y18T21 T3+232.31Y18T23T3+165.29Y18T4+ 234.30Y18T17T4+154.02Y18T5+207.32Y18T1T5+ 224.64Y18T6T5+172.67Y18T14T5+205.62Y18T24T5+ $52.97 \mathrm{Y} 18 \mathrm{~T} 6+145.41 \mathrm{Y} 18 \mathrm{~T} 1 \mathrm{~T} 6+159.61 \mathrm{Y} 18 \mathrm{~T} 2 \mathrm{~T} 6+$ 135.18Y18T5T6+143.42Y18T7T6+116.72Y18T9T6+ 144.56Y18T10T6+136.89Y18T11 T6+159.04Y18T14T6+ $149.67 \mathrm{Y} 18 \mathrm{~T} 15 \mathrm{~T} 6+152.22 \mathrm{Y} 18 \mathrm{~T} 20 \mathrm{~T} 6+130.43 \mathrm{Y} 18 \mathrm{~T} 7+$ $199.08 \mathrm{Y} 18 \mathrm{~T} 6 \mathrm{~T} 7+190.28 \mathrm{Y} 18 \mathrm{~T} 10 \mathrm{~T} 7+168.13 \mathrm{Y} 18 \mathrm{~T} 14 \mathrm{~T} 7+$ $190.85 \mathrm{Y} 18 \mathrm{~T} 19 \mathrm{~T} 7+749.48 \mathrm{Y} 18 \mathrm{~T} 8+459.23 \mathrm{Y} 18 \mathrm{~T} 14 \mathrm{~T} 8+$ $804.86 \mathrm{Y} 18 \mathrm{~T} 15 \mathrm{~T} 8+806.84 \mathrm{Y} 18 \mathrm{~T} 22 \mathrm{~T} 8+123.82 \mathrm{Y} 18 \mathrm{~T} 9+$ $160.74 \mathrm{Y} 18 \mathrm{~T} 3 \mathrm{~T} 9+148.82 \mathrm{Y} 18 \mathrm{~T} 6 \mathrm{~T} 9+138.31 \mathrm{Y} 18 \mathrm{~T} 11 \mathrm{~T} 9+$ 182.61Y18T14T9+229.19Y18T15T9+162.73Y18T16T9+ 171.54Y18T22T9+181.48Y18T23T9+82.11Y18T10+ $159.89 \mathrm{Y} 18 \mathrm{~T} 6 \mathrm{~T} 10+135.75 \mathrm{Y} 18 \mathrm{~T} 7 \mathrm{~T} 10+135.75 \mathrm{Y} 18 \mathrm{~T} 1410+$ $138.88 \mathrm{Y} 18 \mathrm{~T} 1910+110.26 \mathrm{Y} 18 \mathrm{~T} 11+152.79 \mathrm{Y} 18 \mathrm{~T} 3 \mathrm{~T} 11+$ $160.46 \mathrm{Y} 18 \mathrm{~T} 6 \mathrm{~T} 11+129.79 \mathrm{Y} 18 \mathrm{~T} 9 \mathrm{~T} 11+180.91 \mathrm{Y} 18 \mathrm{~T} 1411+$ $178.92 \mathrm{Y} 18 \mathrm{~T} 1511+165.29 \mathrm{Y} 18 \mathrm{~T} 1611+145.98 \mathrm{Y} 18 \mathrm{~T} 1711+$ $174.38 \mathrm{Y} 18 \mathrm{~T} 2111+236.57 \mathrm{Y} 18 \mathrm{~T} 2211+373.74 \mathrm{Y} 18 \mathrm{~T} 12+$ $255.22 \mathrm{Y} 18 \mathrm{~T} 13+316.09 \mathrm{Y} 18 \mathrm{~T} 1413+56.75 \mathrm{Y} 18 \mathrm{~T} 14+$ 102.24Y18T1T14+117.58Y18T2 T14+135.18Y18T3T14+ 108.77Y18T5T14+127.80Y18T6T14+123.82Y18T7T14+ $126.95 \mathrm{Y} 18 \mathrm{~T} 8 \mathrm{~T} 14+105.08 \mathrm{Y} 18 \mathrm{~T} 9 \mathrm{~T} 14+117.58 \mathrm{Y} 18 \mathrm{~T} 1014+$ $111.90 \mathrm{Y} 18 \mathrm{~T} 1114+123.82 \mathrm{Y} 18 \mathrm{~T} 1314+108.20 \mathrm{Y} 18 \mathrm{~T} 1514+$ $151.66 \mathrm{Y} 18 \mathrm{~T} 1614+135.18 \mathrm{Y} 18 \mathrm{~T} 1914+102.24 \mathrm{Y} 18 \mathrm{~T} 2014+$ 135.47Y18T2214+153.08Y18T2314+112.18Y18T2414+ $109.91 \mathrm{Y} 18 \mathrm{~T} 2514+110.76 \mathrm{Y} 18 \mathrm{~T} 15+157.62 \mathrm{Y} 18 \mathrm{~T} 2 \mathrm{~T} 15+$ $165.00 \mathrm{Y} 18 \mathrm{~T} 6 \mathrm{~T} 15+149.95 \mathrm{Y} 18 \mathrm{~T} 8 \mathrm{~T} 15+169.83 \mathrm{Y} 18 \mathrm{~T} 9 \mathrm{~T} 15+$ $156.48 \mathrm{Y} 18 \mathrm{~T} 1115+126.38 \mathrm{Y} 18 \mathrm{~T} 1415+152.79 \mathrm{Y} 18 \mathrm{~T} 1615+$ $138.59 \mathrm{Y} 18 \mathrm{~T} 2015+178.35 \mathrm{Y} 18 \mathrm{~T} 2215+184.88 \mathrm{Y} 18 \mathrm{~T} 16+$ 226.63Y18T9T16+223.51Y18T1116+264.69Y18T1416+ $247.65 \mathrm{Y} 18 \mathrm{~T} 1516+248.78 \mathrm{Y} 18 \mathrm{~T} 2316+387.94 \mathrm{Y} 18 \mathrm{~T} 17+$ 433.38Y18T4T17+323.48Y18T1117+475.42Y18T2117+ $390.50 \mathrm{Y} 18 \mathrm{~T} 19+455.25 \mathrm{Y} 18 \mathrm{~T} 2 \mathrm{~T} 19+447.30 \mathrm{Y} 18 \mathrm{~T} 7 \mathrm{~T} 19+$ $449.86 \mathrm{Y} 18 \mathrm{~T} 1019+364.94 \mathrm{Y} 18 \mathrm{~T} 1419+128.44 \mathrm{Y} 18 \mathrm{~T} 20+$ $184.03 \mathrm{Y} 18 \mathrm{~T} 2 \mathrm{~T} 20+153.08 \mathrm{Y} 18 \mathrm{~T} 6 \mathrm{~T} 20+176.93 \mathrm{Y} 18 \mathrm{~T} 1420+$ $138.31 \mathrm{Y} 18 \mathrm{~T} 1520+238.56 \mathrm{Y} 18 \mathrm{~T} 2220+752.88 \mathrm{Y} 18 \mathrm{~T} 21+$ 447.30Y18T3T21+803.44Y18T1121+813.38Y18T1721+ $816.22 \mathrm{Y} 18 \mathrm{~T} 2221+149.10 \mathrm{Y} 18 \mathrm{~T} 22+191.98 Y 18 \mathrm{~T} 2 \mathrm{~T} 22+$ $157.62 \mathrm{Y} 18 \mathrm{~T} 8 \mathrm{~T} 22+146.26 \mathrm{Y} 18 \mathrm{~T} 9 \mathrm{~T} 22+234.02 \mathrm{Y} 18 \mathrm{~T} 1122+$ $173.52 \mathrm{Y} 18 \mathrm{~T} 1422+198.23 \mathrm{Y} 18 \mathrm{~T} 1522+258.72 \mathrm{Y} 18 \mathrm{~T} 2022+$ $170.40 \mathrm{Y} 18 \mathrm{~T} 2122+166.14 \mathrm{Y} 18 \mathrm{~T} 2322+227.91 \mathrm{Y} 18 \mathrm{~T} 23+$ $280.02 Y 18 \mathrm{~T} 3 \mathrm{~T} 23+279.74 \mathrm{Y} 18 \mathrm{~T} 9 \mathrm{~T} 23+314.67 \mathrm{Y} 18 \mathrm{~T} 1423+$ $283.15 Y 18 \mathrm{~T} 1623+289.68 \mathrm{Y} 18 \mathrm{~T} 2223+264.03 \mathrm{Y} 18 \mathrm{~T} 24+$ 325.18Y18T5T24+352.44Y18T1424+393.34Y18T25+ $152.22 \mathrm{Y} 18 \mathrm{~T} 1425+376.02 \mathrm{Y} 19 \mathrm{~T} 2+351.02 \mathrm{Y} 19 \mathrm{~T} 14 \mathrm{~T} 2+$ $356.14 \mathrm{Y} 19 \mathrm{~T} 18 \mathrm{~T} 2+716.25 \mathrm{Y} 19 \mathrm{~T} 7+784.98 \mathrm{Y} 19 \mathrm{~T} 10 \mathrm{~T} 7+$ 471.16Y19T14T7+469.45Y19T18T7+721.08Y19T10+ 780.15Y19T7T10+467.18Y19T1410+467.18Y19T1810+ $397.88 \mathrm{Y} 19 \mathrm{~T} 14+329.16 \mathrm{Y} 19 \mathrm{~T} 2 \mathrm{~T} 14+441.62 \mathrm{Y} 19 \mathrm{~T} 7 \mathrm{~T} 14+$ 442.47Y19T1014+357.56Y19T1814+738.40Y19T18+ 341.65Y19T2T18+447.30Y19T7T18+449.86Y19T1018+ 


\section{APPENDIX B: PROBLEM FORMULATION FILE EFFICIENT PASSENGERS NETWORK}

364.94Y19T1418+374.60Y20T2+326.03Y20T6T2+ 432.82Y20T14T2+282.01Y20T15T2+184.60Y20T18T2+ 493.31Y20T22T2+227.91Y20T6+298.77Y20T2T6+ 345.06Y20T14T6+290.82Y20T15T6+168.98Y20T18T6+ 713.98Y20T14+441.34Y20T2T14+324.04Y20T6T14+ 788.95Y20T1514+200.22Y20T1814+828.43Y20T2214+ 721.93Y20T15+807.98Y20T2T15+375.45Y20T6T15+ 781.00Y20T1415+196.24Y20T1815+814.51Y20T2215+ $118.78 \mathrm{Y} 20 \mathrm{~T} 18+169.83 \mathrm{Y} 20 \mathrm{~T} 2 \mathrm{~T} 18+153.08 \mathrm{Y} 20 \mathrm{~T} 6 \mathrm{~T} 18+$ 176.93Y20T1418+138.31Y20T1518+238.56Y20T2218+ 742.66Y20T22+828.14Y20 T2T22+799.74Y $20 \mathrm{~T} 1422+$ 438.78Y20T1522+233.16Y20T1822+714.83Y21T3+ 793.21Y21T11T3+840.36Y21T18T3+727.89Y21T11+ 780.15Y21T3T11+794.06Y21T1711+828.43Y21T1811+ 797.19Y21T2211+730.73Y21T17+791.22Y21T1117+ 835.53Y21T1817+752.88Y21T18+802.30Y21T3T18+ 803.44Y21T1118+813.38Y21T1718+816.22Y21T2218+ 723.92Y21T22+801.16Y21T1122+845.18Y21T1822+ 758.85Y22T2+202.21Y22T14T2+810.54Y22T15T2+ $249.64 \mathrm{Y} 22 \mathrm{~T} 18 \mathrm{~T} 2+811.96 \mathrm{Y} 22 \mathrm{~T} 20 \mathrm{~T} 2+714.54 \mathrm{Y} 22 \mathrm{~T} 8+$ 457.24Y22T14T8+792.93Y22T15T8+841.78Y22T18T8+ 732.44Y22T9+789.24Y22T11T9+251.62Y22T14T9+ 799.46Y22T15T9+230.32Y22T18T9+807.70Y22T23T9+ 726.47Y22T11+795.20Y22T9T11+349.32Y22T1411+ 791.79Y22T1511+238.84Y22T1811+798.61Y22T $2111+$ 130.14Y22T14+165.29Y22T2T14+120.42Y22T8T14+ $159.89 \mathrm{Y} 22 \mathrm{~T} 9 \mathrm{~T} 14+209.02 \mathrm{Y} 22 \mathrm{~T} 1114+153.08 \mathrm{Y} 22 \mathrm{~T} 1514+$ $180.06 \mathrm{Y} 22 \mathrm{~T} 1814+192.84 \mathrm{Y} 22 \mathrm{~T} 2014+154.50 \mathrm{Y} 22 \mathrm{~T} 2314+$ $377.15 \mathrm{Y} 22 \mathrm{~T} 15+318.93 \mathrm{Y} 22 \mathrm{~T} 2 \mathrm{~T} 15+257.02 \mathrm{Y} 22 \mathrm{~T} 8 \mathrm{~T} 15+$ 451.84Y22T9T15+438.21Y22T1115+181.19Y22T1415+ 218.68Y22T1815+456.39Y22T2015+165.67Y22T18+ 206.18Y22T2T18+171.82Y22T8T18+160.46Y22T9T18+ $234.02 \mathrm{Y} 22 \mathrm{~T} 1118+187.72 \mathrm{Y} 22 \mathrm{~T} 1418+198.23 \mathrm{Y} 22 \mathrm{~T} 1518+$ 258.72Y22T2018+184.60Y22T2118+180.34Y22T2318+ 742.66Y22T20+473.14Y22T2T20+799.74Y22T1420+ 438.78Y22T1520+233.16Y22T1820+723.92Y22T $21+$ $801.16 \mathrm{Y} 22 \mathrm{~T} 1121+845.18 \mathrm{Y} 22 \mathrm{~T} 1821+722.78 \mathrm{Y} 22 \mathrm{~T} 23+$ 817.35Y22T9T23+483.08Y22T1423+316.66Y22T1823+ 717.95Y23 $3+811.39 \mathrm{Y} 23 \mathrm{~T} 9 \mathrm{~T} 3+487.63 \mathrm{Y} 23 \mathrm{~T} 14 \mathrm{~T} 3+$ 368.63Y23T $18 \mathrm{~T} 3+738.12 \mathrm{Y} 23 \mathrm{~T} 9+791.22 \mathrm{Y} 23 \mathrm{~T} 3 \mathrm{~T} 9+$ 477.97Y23T14T9+794.63Y23T16 T9+348.18Y23T18T9+ 802.02Y23T22T9+752.32Y23T14+453.26Y23T3T14+ 463.77Y23T9T14+471.16Y23T1614+312.12Y23T1814+ 453.55Y23T2214+720.22Y23T16+812.52Y23T9T16+ 503.25Y23T1416+369.48Y23T1816+285.89Y23T18+ $336.82 \mathrm{Y} 23 \mathrm{~T} 3 \mathrm{~T} 18+336.54 \mathrm{Y} 23 \mathrm{~T} 9 \mathrm{~T} 18+314.67 \mathrm{Y} 23 \mathrm{~T} 1418+$ $339.95 Y 23 \mathrm{~T} 1618+289.68 \mathrm{Y} 23 \mathrm{~T} 2218+722.78 \mathrm{Y} 23 \mathrm{~T} 22+$ 817.35Y23T9T22+483.08Y23T1422+316.66Y23T1822+ 713.12Y24T5+804.00Y24T14T5+339.95Y24T18T5+ 733.29Y24T14+783.84Y24T5T14+347.05Y24T1814+ $264.03 \mathrm{Y} 24 \mathrm{~T} 18+325.18 \mathrm{Y} 24 \mathrm{~T} 5 \mathrm{~T} 18+352.44 \mathrm{Y} 24 \mathrm{~T} 1418+$ 75.39Y25T14+175.80Y25T1814+393.34Y25T18+ 152.22Y25T1418

ST

Y1T6-Y1T6T5 > $=0$

Y6T5-Y1T6T5 > $=0$

Y1T14-Y1T14T5 $>=0$

Y14T5-Y1T14T5 $>=0$

Y1T18-Y1T18T5 $>=0$

Y18T5-Y1T18T5 $>=0$

Y1T5-Y1T5T6 $>=0$

Y5T6-Y1T5T6 > $=0$

Y1T14-Y1T14T6 $>=0$

Y14T6-Y1T14T6 > $=0$

Y1T18-Y1T18T6 > $=0$
Y18T6-Y1T18T6>=0

Y1T5-Y1T5T14> $>0$

Y5T14-Y1T5T14 > 0

Y1T6-Y1T6T14 > $=0$

Y6T14-Y1T6T14 > $=0$

$\mathrm{Y} 1 \mathrm{~T} 18-\mathrm{Y} 1 \mathrm{~T} 18 \mathrm{~T} 14>=0$

Y18T14-Y1T18T14 $>=0$

Y1T5-Y1T5T18 > $=0$

Y5T18-Y1T5T18 > $=0$

Y1T6-Y1T6T18 > $=0$

Y6T18-Y1T6T18 $>=0$

Y1T14-Y1T14T18 $>=0$

Y14T18-Y1T14T18 > $=0$

Y2T14-Y2T14T6 $>=0$

Y14T6-Y2T14T6 > $=0$

Y2T15-Y2T15T6 > $=0$

Y15T6-Y2T15T6 > $=0$

Y2T18-Y2T18T6 > $=0$

Y18T6-Y2T18T6 > $=0$

Y2T20-Y2T20T6 $>=0$

Y20T6-Y2T20T6 > $=0$

Y2T6-Y2T6T14 $>=0$

Y6T14-Y2T6T14 >=0

Y2T15-Y2T15T14 > $=0$

Y15T14-Y2T15T14 $>=0$

Y2T18-Y2T18T14 $>=0$

$\mathrm{Y} 18 \mathrm{~T} 14-\mathrm{Y} 2 \mathrm{~T} 18 \mathrm{~T} 14>=0$

Y2T19-Y2T19T14 $>=0$

Y19T14-Y2T19T14 $>=0$

Y2T20-Y2T20T14 $>=0$

Y20T14-Y2T20T14 $>=0$

Y2T22-Y2T22T14 $>=0$

$\mathrm{Y} 22 \mathrm{~T} 14-\mathrm{Y} 2 \mathrm{~T} 22 \mathrm{~T} 14>=0$

Y2T6-Y2T6T15 > $=0$

Y6T15-Y2T6T15 > $=0$

Y2T14-Y2T14T15 > $=0$

Y14T15-Y2T14T15 > $=0$

$\mathrm{Y} 2 \mathrm{~T} 18-\mathrm{Y} 2 \mathrm{~T} 18 \mathrm{~T} 15>=0$

$\mathrm{Y} 18 \mathrm{~T} 15-\mathrm{Y} 2 \mathrm{~T} 18 \mathrm{~T} 15>=0$

Y2T20-Y2T20T15 $>=0$

Y20T15-Y2T20T15 $>=0$

Y2T22-Y2T22T15 $>=0$

Y22T15-Y2T22T15 $>=0$

Y2T6-Y2T6T18 $>=0$

Y6T18-Y2T6T18 > $=0$

Y2T14Y2T14T18 $>=0$

Y14T18-Y2T14T18 >- 0

Y2T15-Y2T15T18 $>=0$

Y15T18-Y2T15T18 > $=0$

Y2T19-Y2T19T18 $>=0$

Y19T18-Y2T19T18 > $=0$

Y2T20-Y2T20T18 > $=0$

Y20T18-Y2T20T18 $>=0$

$\mathrm{Y} 2 \mathrm{~T} 22-\mathrm{Y} 2 \mathrm{~T} 22 \mathrm{~T} 18>=0$

Y22T18-Y2T22T18 > $=0$

Y2T14-Y2T14T19 > $=0$

Y14T19-Y2T14T19>=0

Y2T18-Y2T18T19 $>=0$

Y18T19-Y2T18T19 $>=0$

Y2T6-Y2T6T20 > $=0$

Y6T20-Y2T6T20 > $=0$

Y2T14-Y2T14T20> $>0$

$\mathrm{Y} 14 \mathrm{~T} 20-\mathrm{Y} 2 \mathrm{~T} 14 \mathrm{~T} 20>0$

Y2T15-Y2T15T20 $>=0$

Y15T20-Y2T15T20 > $=0$ 
APPENDIX B: PROBLEM FORMULATION FILE EFFICIENT PASSENGERS NETWORK

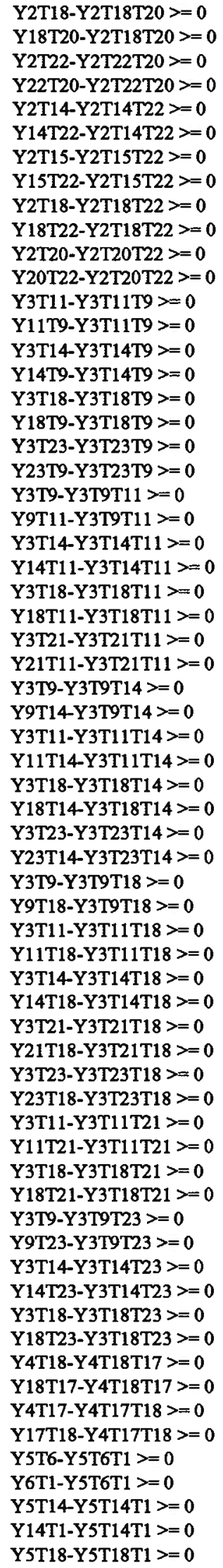

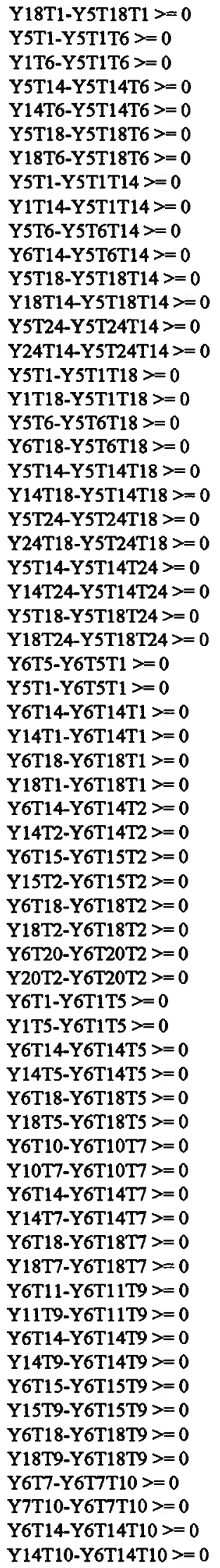


APPENDIX B: PROBLEM FORMULATION FILE EFFICIENT PASSENGERS NETWORK

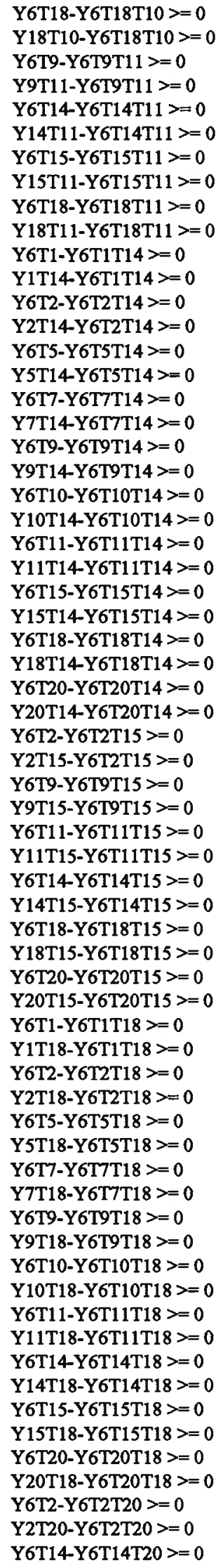

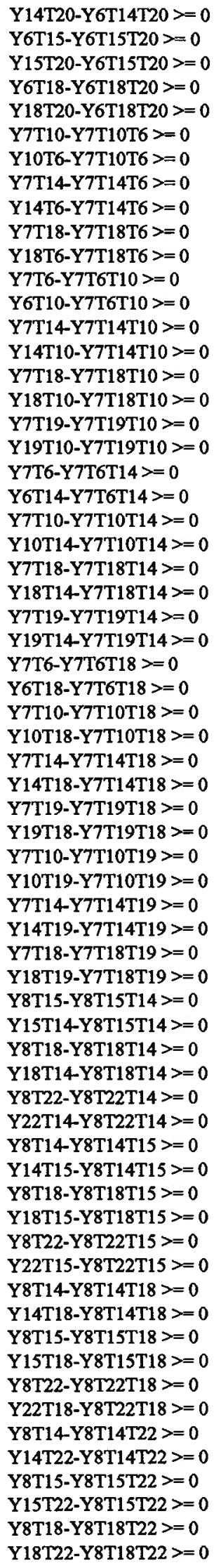




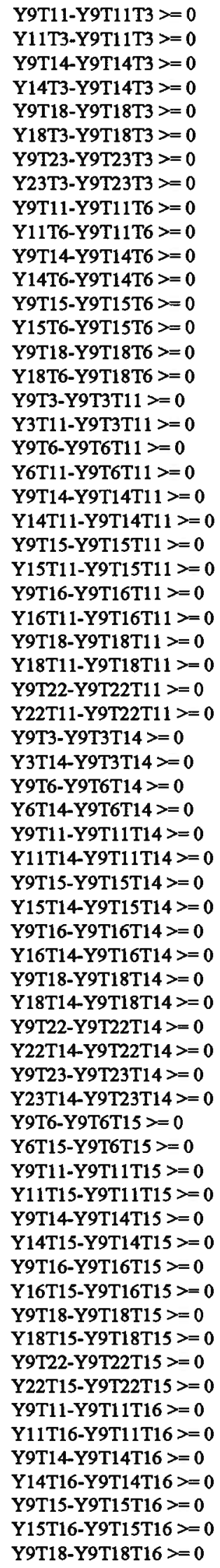

\section{APPENDIX B: PROBLEM FORMULATION FILE EFFICIENT PASSENGERS NETWORK}

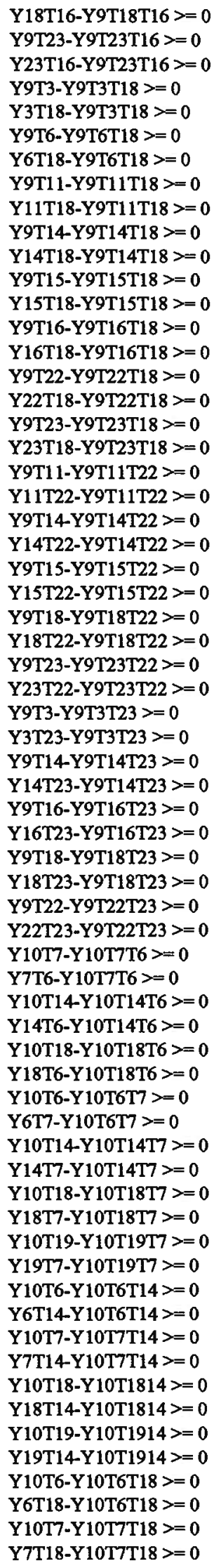




\section{APPENDIX B: PROBLEM FORMULATION FILE EFFICIENT PASSENGERS NETWORK}

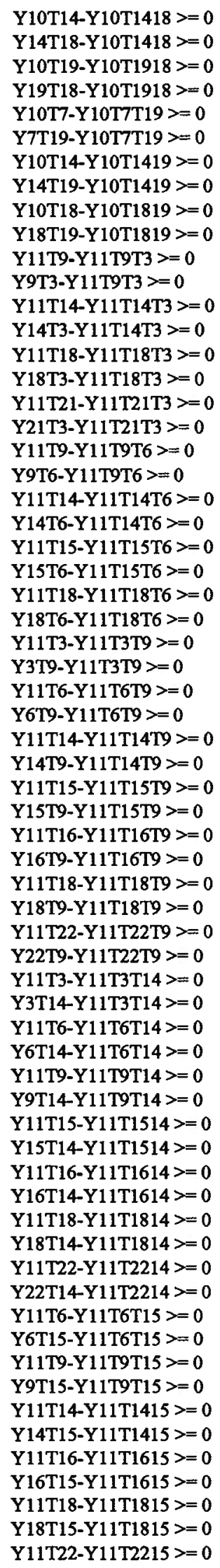

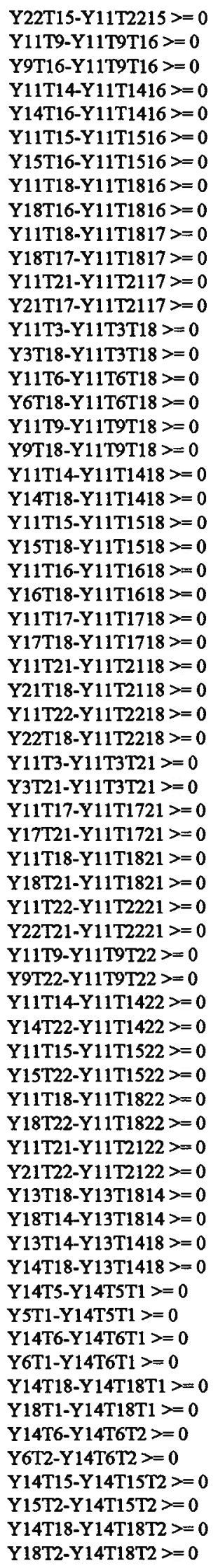




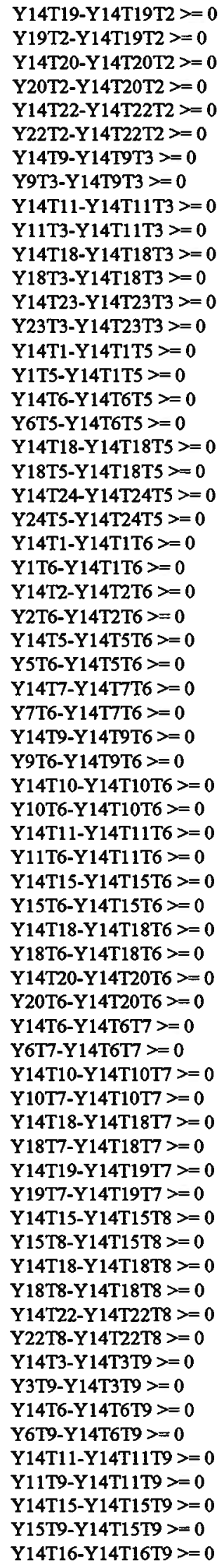

APPENDIX B: PROBLEM FORMULATION FILE EFFICIENT PASSENGERS NETWORK

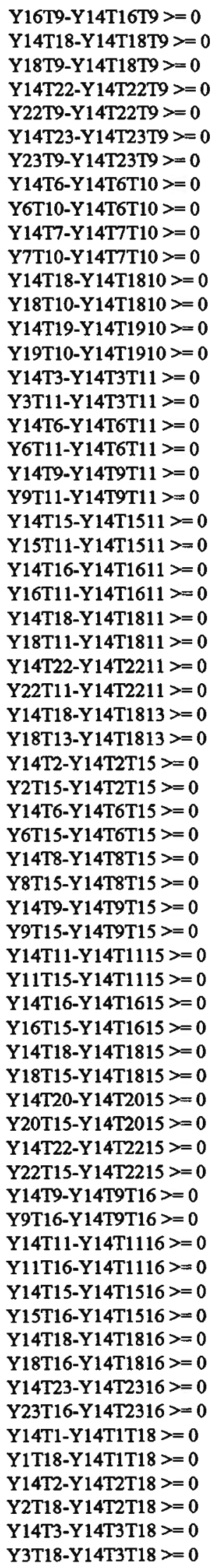




\section{APPENDIX B: PROBLEM FORMULATION FILE EFFICIENT PASSENGERS NETWORK}

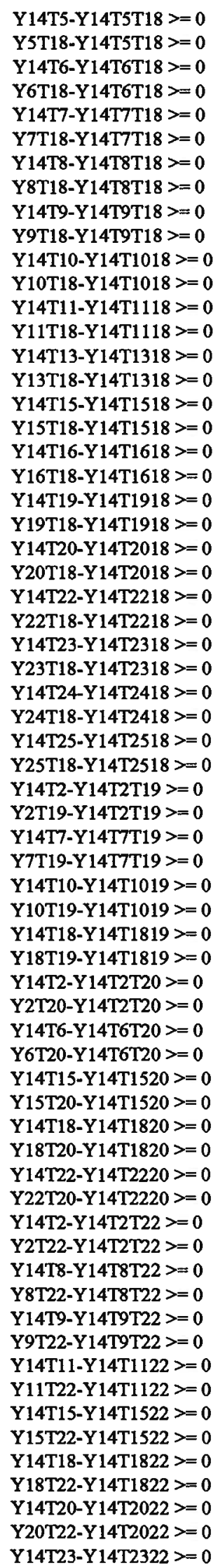

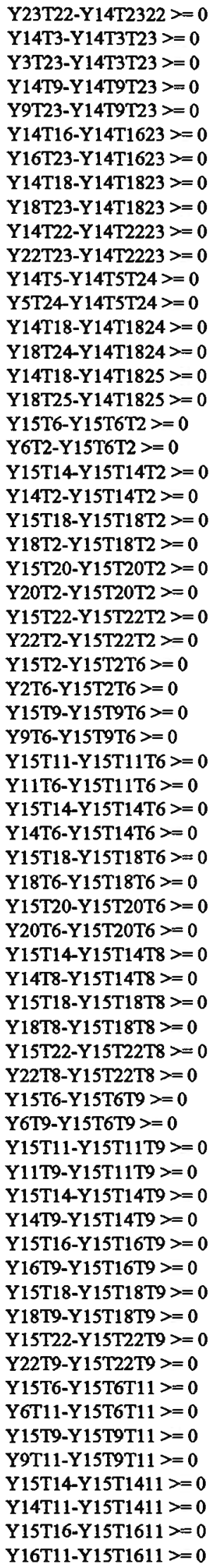




\section{APPENDIX B: PROBLEM FORMULATION FILE EFFICIENT PASSENGERS NETWORK}

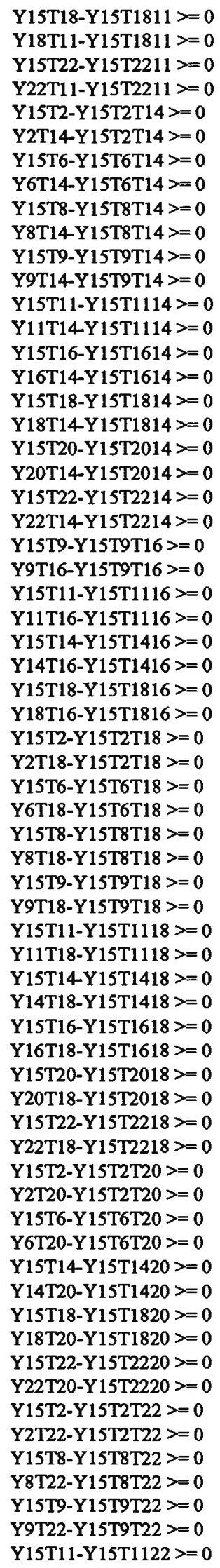

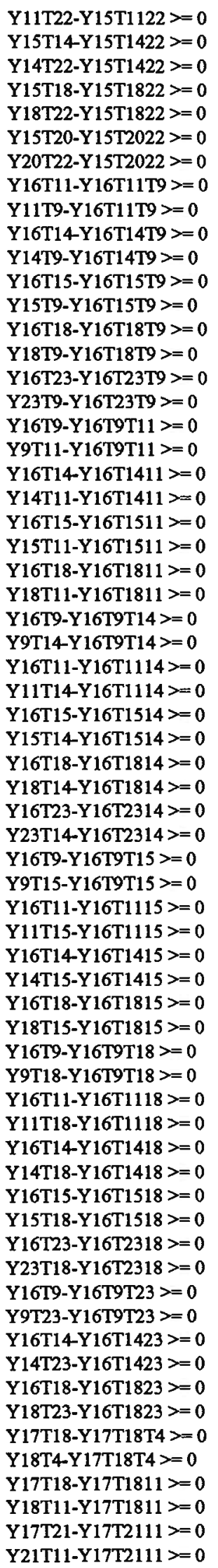


APPENDIX B: PROBLEM FORMULATION FILE EFFICIENT PASSENGERS NETWORK

Y17T4-Y17T4T18 >=0 Y4T18-Y17T4T18>=0 Y17T11-Y17T1118>=0 $Y 11 T 18-Y 17 T 1118>=0$ $\mathrm{Y} 17 \mathrm{~T} 21-\mathrm{Y} 17 \mathrm{~T} 2118>=0$ Y21T18-Y17T2118 $>=0$ $\mathrm{Y} 17 \mathrm{~T} 11-\mathrm{Y} 17 \mathrm{~T} 1121>=0$ Y11T21-Y17T1121 >=0 Y17T18-Y17T1821 >=0 Y18T21-Y17T1821 > $=0$ Y18T5-Y18T5T1 >=0 Y5T1-Y18T5T1 >=0 Y18T6-Y18T6T1 $>=0$ $\mathrm{Y} 6 \mathrm{~T} 1-\mathrm{Y} 18 \mathrm{~T} 6 \mathrm{~T} 1>=0$ Y18T14-Y18T14T1 > 0 Y14T1-Y18T14T1 >= 0 $\mathrm{Y} 18 \mathrm{~T} 6-\mathrm{Y} 18 \mathrm{~T} 6 \mathrm{~T} 2>=0$ Y6T2-Y18T6T2>=0 $\mathrm{Y} 18 \mathrm{~T} 14-\mathrm{Y} 18 \mathrm{~T} 14 \mathrm{~T} 2>=0$ Y14T2-Y18T14T2 $>=0$ Y18T15-Y18T15T2 > $=0$ $\mathrm{Y} 15 \mathrm{~T} 2-\mathrm{Y} 18 \mathrm{~T} 15 \mathrm{~T} 2>=0$ Y18T19-Y18T19T2 $>=0$ $\mathrm{Y} 19 \mathrm{~T} 2-\mathrm{Y} 18 \mathrm{~T} 19 \mathrm{~T} 2>=0$ Y18T20-Y18T20T2 $>=0$ Y20T2-Y18T20T2 $>=0$ $\mathrm{Y} 18 \mathrm{~T} 22-\mathrm{Y} 18 \mathrm{~T} 22 \mathrm{~T} 2>=0$ Y22T2-Y18T22T2 > 0

Y18T9-Y18T9T3 $>=0$

Y9T3-Y18T9T3 $>=0$

$\mathrm{Y} 18 \mathrm{~T} 11-\mathrm{Y} 18 \mathrm{~T} 11 \mathrm{~T} 3>=0$ Y11T3-Y18T11T3 $>=0$ $Y 18 T 14-Y 18 T 14 T 3>=0$ Y14T3-Y18T14T3 $>=0$ Y18T21-Y18T21T3 $>=0$ Y21T3-Y18T21T3 > $=0$ Y18T23-Y18T23T3 $>=0$ $\mathrm{Y} 23 \mathrm{~T} 3-\mathrm{Y} 18 \mathrm{~T} 23 \mathrm{~T} 3>=0$ $\mathrm{Y} 18 \mathrm{~T} 17-\mathrm{Y} 18 \mathrm{~T} 17 \mathrm{~T} 4>=0$ Y17T4-Y18T17T4 $>=0$ Y18T1-Y18T1T5 $>=0$ Y1T5-Y18T1T5 > = 0 Y18T6-Y18T6T5 > $=0$ Y6T5-Y18T6T5 $>=0$ Y18T14-Y18T14T5 >=0 Y14T5-Y18T14T5 $>=0$ Y18T24-Y18T24T5 $>=0$ Y24T5-Y $18 \mathrm{~T} 24 \mathrm{~T} 5>=0$ $\mathrm{Y} 18 \mathrm{~T} 1-\mathrm{Y} 18 \mathrm{~T} 1 \mathrm{~T} 6>=0$ Y1T6-Y18T1T6 >= 0 Y18T2-Y18T2T6 $>=0$ Y2T6-Y18T2T6 > $=0$ Y18T5-Y18T5T6 $>=0$ Y5T6-Y18T5T6 > $=0$ Y18T7-Y18T7T6 $>=0$ Y7T6-Y18T7T6 > $=0$ Y18T9-Y18T9T6 > = 0 Y9T6-Y18T9T6 > = 0 Y18T10-Y18T10T6 > $=0$ Y10T6-Y18T10T6 > $=0$ Y18T11-Y18T11T6 >= 0 Y11T6-Y18T11T6 $>=0$ Y18T14-Y18T14T6 $>=0$ Y14T6-Y18T14T6 > $=0$ Y18T15-Y18T15T6 >= 0

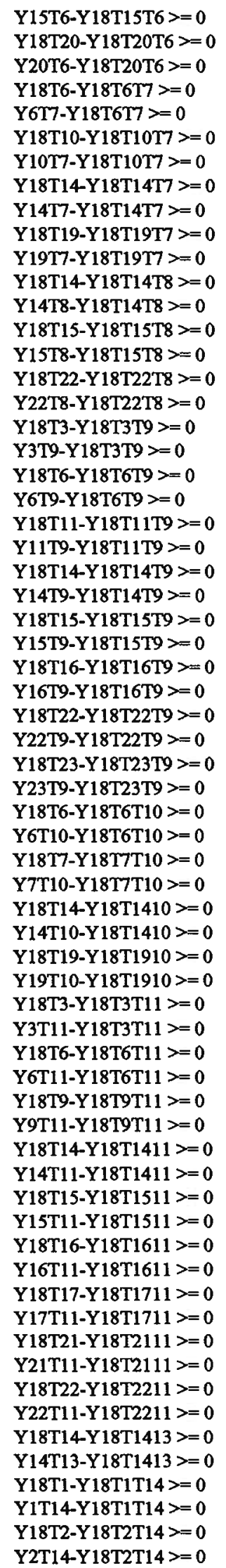




\section{APPENDIX B: PROBLEM FORMULATION FILE EFFICIENT PASSENGERS NETWORK}

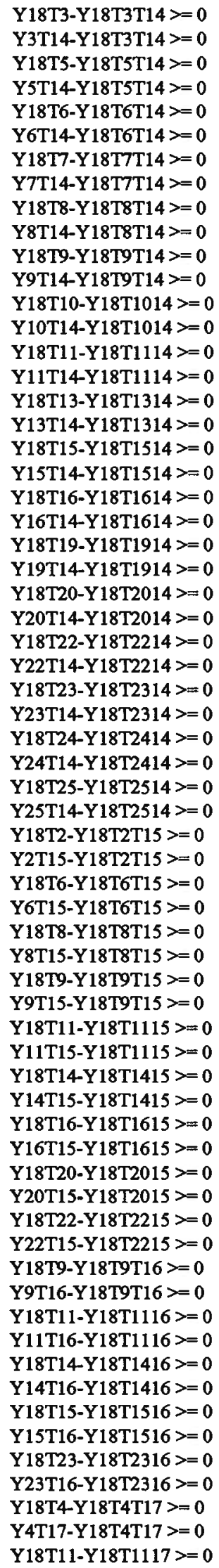

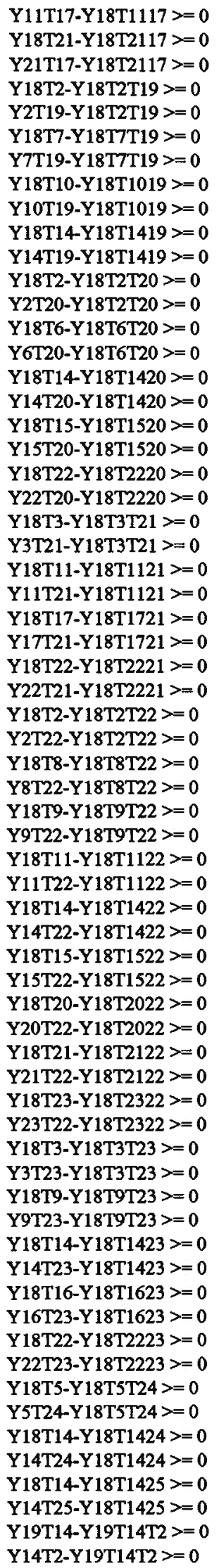




\section{APPENDIX B: PROBLEM FORMULATION FILE EFFICIENT PASSENGERS NETWORK}

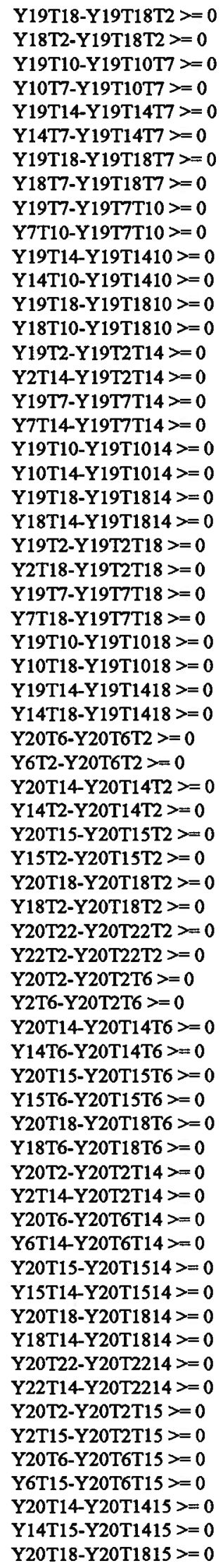

Y20T22-Y20T1815 > $=0$

Y22T15-Y20T2215 > $=0$

Y20T2-Y20T2T18 > $=0$

Y2T18-Y20T2T18 > $=0$

Y20T6-Y20T6T18 $>=0$

Y6T18-Y20T6T18 > $=0$

Y20T14-Y20T1418 > $=0$

Y 14T18-Y20T1418 >= 0

Y20T15-Y20T1518 $>=0$

Y15T18-Y20T1518>=0

Y20T22-Y20T2218 $>=0$

Y22T18-Y20T2218 > $=0$

Y20T2-Y20T2T22 > $=0$

Y2T22-Y20T2T22 > $=0$

Y20T14-Y20T1422 $>=0$

Y14T22-Y20T1422 $>=0$

Y20T15-Y20T1522> $>0$

$\mathrm{Y} 15 \mathrm{~T} 22-\mathrm{Y} 20 \mathrm{~T} 1522>=0$

Y20T18-Y20T1822 > $=0$

$Y 18 T 22-Y 20 T 1822>=0$

Y21T11-Y21T11T3 $>=0$

Y11T3-Y21T11T3 $>=0$

Y21T18-Y21T18T3 $>=0$

Y18T3-Y21T18T3 $>=0$

Y21T3-Y21T3T11 $>=0$

Y3T11-Y21T3T11 $>=0$

Y21T17-Y21T1711 $>=0$

Y17T11-Y21T1711 $>=0$

Y21T18-Y21T1811 > $=0$

$Y 18 T 11-Y 21 T 1811>=0$

Y21T22-Y21T2211 > $=0$

Y22T11-Y21T2211 > $=0$

Y21T11-Y21T1117 $>=0$

$Y 11 T 17-Y 21 T 1117>=0$

Y21T18-Y21T1817 > $=0$

$Y 18 T 17-Y 21 T 1817>=0$

Y21T3-Y21T3T18 > $=0$

Y3T18-Y21T3T18 $>=0$

Y21T11-Y21T1118>=0

Y11T18-Y21T1118 $>=0$

Y21T17-Y21T1718 > = 0

Y17T18-Y21T1718 > $=0$

Y21T22-Y21T2218 > 0

Y22T18-Y21T2218>= 0

Y21T11-Y21T1122 > $=0$

Y11T22-Y21T1122 $>=0$

Y21T18-Y21T1822 $>=0$

$\mathrm{Y} 18 \mathrm{~T} 22-\mathrm{Y} 21 \mathrm{~T} 1822>=0$

$\mathrm{Y} 22 \mathrm{~T} 14-\mathrm{Y} 22 \mathrm{~T} 14 \mathrm{~T} 2>=0$

$\mathrm{Y} 14 \mathrm{~T} 2-\mathrm{Y} 22 \mathrm{~T} 14 \mathrm{~T} 2>=0$

$\mathrm{Y} 22 \mathrm{~T} 15-\mathrm{Y} 22 \mathrm{~T} 15 \mathrm{~T} 2>=0$

$\mathrm{Y} 15 \mathrm{~T} 2-\mathrm{Y} 22 \mathrm{~T} 15 \mathrm{~T} 2>0$

Y22T18-Y22T18T2 $>=0$

$\mathrm{Y} 18 \mathrm{~T} 2-\mathrm{Y} 22 \mathrm{~T} 18 \mathrm{~T} 2>=0$

$\mathrm{Y} 22 \mathrm{~T} 20-\mathrm{Y} 22 \mathrm{~T} 20 \mathrm{~T} 2>=0$

Y20T2-Y22T20T2 $>=0$

Y22T14-Y22T14T8 $>0$

Y14T8-Y22T14T8 $>=0$

$Y 22 \mathrm{~T} 15-\mathrm{Y} 22 \mathrm{~T} 15 \mathrm{~T} 8>=0$

Y15T8-Y22T15T8 $>=0$

Y22T18-Y22T18T8 > $=0$

$\mathrm{Y} 18 \mathrm{~T} 8-\mathrm{Y} 22 \mathrm{~T} 18 \mathrm{~T} 8>=0$

Y22T11-Y22T11T9 $>=0$

Y11T9-Y22T11T9 $>=0$ 


\section{APPENDIX B: PROBLEM FORMULATION FILE EFFICIENT PASSENGERS NETWORK}

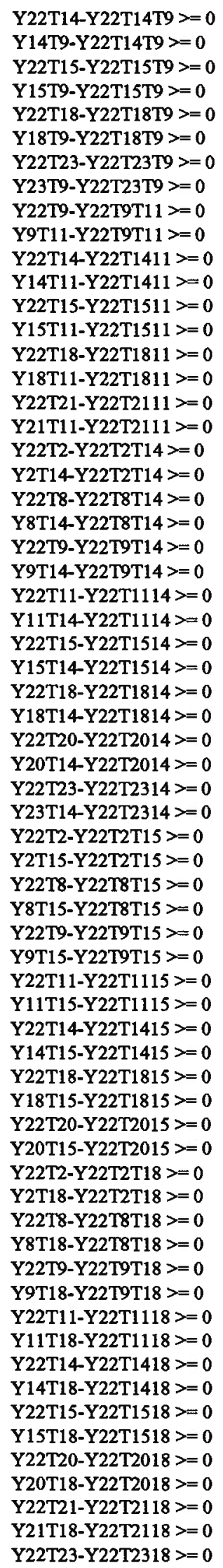

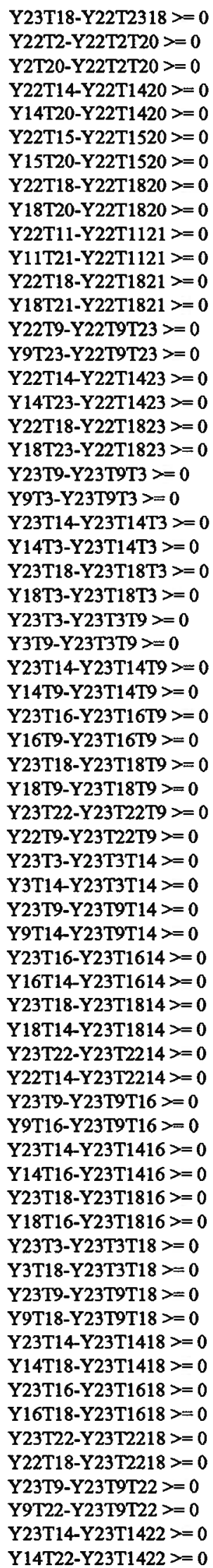




\section{APPENDIX B: PROBLEM FORMULATION FILE EFFICIENT PASSENGERS NETWORK}

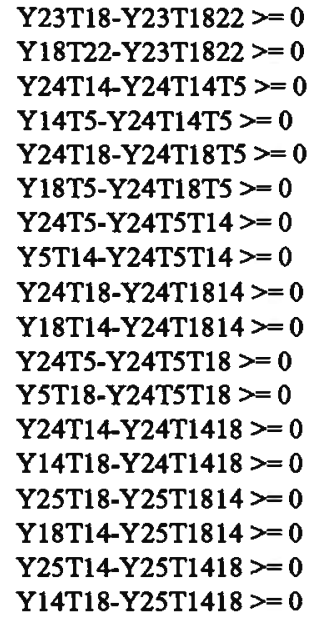

Y6T7T14+Y6T9T14+Y6T10T14+Y6T11T14+ Y6T15T14+Y6T18T14+Y6T20T14=1 Y6T15+Y6T2T15+Y6T9T15+Y6T11T15+ $\mathrm{Y} 6 \mathrm{~T} 14 \mathrm{~T} 15+\mathrm{Y} 6 \mathrm{~T} 18 \mathrm{~T} 15+\mathrm{Y} 6 \mathrm{~T} 20 \mathrm{~T} 15=1$ Y6T18+Y6T1T18+Y6T2T18+Y6T5T18+ Y6T7T18+Y6T9T18+Y6T10T18+Y6T11T18+ Y6T14T18+Y6T15T18+Y6T20T18=1 Y6T20+Y6T2T20+Y6T14T20+Y6T15T20+ $\mathrm{Y} 6 \mathrm{~T} 18 \mathrm{~T} 20=1$ Y7T6+Y7T10T6+Y7T14T6+Y7T18T6=1 Y7T10+Y7T6T10+Y7T14T10+Y7T18T10+ Y7T19T10=1 Y7T14+Y7T6T14+Y7T10T14+Y7T18T14+ Y7T19T14=1 Y7T18+Y7T6T18+Y7T10T18+Y7T14T18+ Y7T19T18=1 Y7T19+Y7T10T19+Y7T14T19+Y7T18T19=1 Y8T14+Y8T15T14+Y8T18T14+Y8T22T14=1 $\mathrm{Y} 8 \mathrm{~T} 15+\mathrm{Y} 8 \mathrm{~T} 14 \mathrm{~T} 15+\mathrm{Y} 8 \mathrm{~T} 18 \mathrm{~T} 15+\mathrm{Y} 8 \mathrm{~T} 22 \mathrm{~T} 15=1$ $\mathrm{Y} 8 \mathrm{~T} 18+\mathrm{Y} 8 \mathrm{~T} 14 \mathrm{~T} 18+\mathrm{Y} 8 \mathrm{~T} 15 \mathrm{~T} 18+\mathrm{Y} 8 \mathrm{~T} 22 \mathrm{~T} 18=1$ $\mathrm{Y} 8 \mathrm{~T} 22+\mathrm{Y} 8 \mathrm{~T} 14 \mathrm{~T} 22+\mathrm{Y} 8 \mathrm{~T} 15 \mathrm{~T} 22+\mathrm{Y} 8 \mathrm{~T} 18 \mathrm{~T} 22=1$ Y9T3+Y9T11T3+Y9T14T3+Y9T18T3+ $\mathrm{Y} 9 \mathrm{~T}_{23 \mathrm{~T}} \mathrm{3}=1$ Y9T6+Y9T11T6+Y9T14T6+Y9T15T6+ Y9T18T6=1 Y9T11+Y9T3T11+Y9T6T11+Y9T14T11+ Y9T15T11+Y9T16T11+Y9T18T11+Y9T22T11=1 Y9T14+Y9T3T14+Y9T6T14+Y9T11T14+ Y9T15T14+Y9T16T14+Y9T18T14+Y9T22T14+ Y9T23T14 $=1$ Y9T15+Y9T6T15+Y9T11T15+Y9T14T15+ Y9T16T15+Y9T18T15+Y9T22T15=1 Y9T16+Y9T11T16+Y9T14T16+Y9T15T16+ Y9T18T16+Y9T23T16=1 Y9T18+Y9T3T18+Y9T6T18+Y9T11T18+ Y9T14T18+Y9T15T18+Y9T16T18+Y9T22T18+ Y9T23T18=1 Y9T22+Y9T11T22+Y9T14T22+Y9T15T22+ Y $9 \mathrm{~T} 18 \mathrm{~T} 22+\mathrm{Y} 9 \mathrm{~T} 23 \mathrm{~T} 22=1$ Y9T23+Y9T3T23+Y9T14T23+Y9T16T23+ $\mathrm{Y} 9 \mathrm{~T} 18 \mathrm{~T} 23+\mathrm{Y} 9 \mathrm{~T} 22 \mathrm{~T} 23=1$ Y10T6+Y10T7T6+Y10T14T6+Y10T18T6=1 $\mathrm{Y} 10 \mathrm{~T} 7+\mathrm{Y} 10 \mathrm{~T} 6 \mathrm{~T} 7+\mathrm{Y} 10 \mathrm{~T} 14 \mathrm{~T} 7+\mathrm{Y} 10 \mathrm{~T} 18 \mathrm{~T} 7+$ $\mathrm{Y} 10 \mathrm{~T} 19 \mathrm{~T} 7=1$ Y10T14+Y10T6T14+Y10T7T14+Y10T1814+ $\mathrm{Y} 10 \mathrm{~T} 1914=1$ $\mathrm{Y} 10 \mathrm{~T} 18+\mathrm{Y} 10 \mathrm{~T} 6 \mathrm{~T} 18+\mathrm{Y} 10 \mathrm{~T} 7 \mathrm{~T} 18+\mathrm{Y} 10 \mathrm{~T} 1418+$ $\mathrm{Y} 10 \mathrm{~T} 1918=1$ $\mathrm{Y} 10 \mathrm{~T} 19+\mathrm{Y} 10 \mathrm{~T} 7 \mathrm{~T} 19+\mathrm{Y} 10 \mathrm{~T} 1419+\mathrm{Y} 10 \mathrm{~T} 1819=1$ Y11T3+Y11T9T3+Y11T14T3+Y11T18T3+ $\mathrm{Y} 11 \mathrm{~T} 21 \mathrm{~T} 3=1$ Y11T6+Y11T9T6+Y11T14T6+Y11T15T6+ Y11T18T6=1 Y11T9+Y11T3T9+Y11T6T9+Y11T14T9+ Y11T15T9+Y11T16T9+Y11T18T9+Y11T22T9=1 Y11T14+Y11T3T14+Y11T6T14+Y11T9T14+ $\mathrm{Y} 11 \mathrm{~T} 1514+\mathrm{Y} 11 \mathrm{~T} 1614+\mathrm{Y} 11 \mathrm{~T} 1814+\mathrm{Y} 11 \mathrm{~T} 2214=1$ Y11T15+Y11T6T15+Y11T9T15+Y11T1415+ Y11T1615+Y11T1815+Y11T2215=1 Y11T16+Y11T9T16+Y11T1416+Y11T1516+ Y11T1816=1 Y11T17+Y11T1817+Y11T2117=1 Y11T18+Y11T3T18+Y11T6T18+Y11T9T18+ Y11T1418+Y11T1518+Y11T1618+Y11T1718+ Y11T2118+Y11T2218=1 


\section{APPENDIX B: PROBLEM FORMULATION FILE EFFICIENT PASSENGERS NETWORK}

$\mathrm{Y} 11 \mathrm{~T} 21+\mathrm{Y} 11 \mathrm{~T} 3 \mathrm{~T} 21+\mathrm{Y} 11 \mathrm{~T} 1721+\mathrm{Y} 11 \mathrm{~T} 1821+$ $Y 11 T 2221=1$

Y11T22+Y11T9T22+Y11T1422+Y11T1522+

$\mathrm{Y} 11 \mathrm{~T} 1822+\mathrm{Y} 11 \mathrm{~T} 2122=1$

$\mathrm{Y} 12 \mathrm{~T} 18=1$

$\mathrm{Y} 13 \mathrm{~T} 14+\mathrm{Y} 13 \mathrm{~T} 1814=1$

$\mathrm{Y} 13 \mathrm{~T} 18+\mathrm{Y} 13 \mathrm{~T} 1418=1$

$\mathrm{Y} 14 \mathrm{~T} 1+\mathrm{Y} 14 \mathrm{~T} 5 \mathrm{~T} 1+\mathrm{Y} 14 \mathrm{~T} 6 \mathrm{~T} 1+\mathrm{Y} 14 \mathrm{~T} 18 \mathrm{~T} 1=1$

$\mathrm{Y} 14 \mathrm{~T} 2+\mathrm{Y} 14 \mathrm{~T} 6 \mathrm{~T} 2+\mathrm{Y} 14 \mathrm{~T} 15 \mathrm{~T} 2+\mathrm{Y} 14 \mathrm{~T} 18 \mathrm{~T} 2+$

$Y 14 T 19 T 2+Y 14 T 20 T 2+Y 14 T 22 T 2=1$

Y14T3+Y14T9T3+Y14T11T3+Y14T18T3+

$Y 14 T 23 T 3=1$

Y14T5+Y14T1T5+Y14T6T5+Y14T18T5+

Y14T24T5=1

Y14T6+Y14T1T6+Y14T2T6+Y14T5T6+

Y14T7T6+Y14T9T6+Y14T10T6+Y14T11T6+

$\mathrm{Y} 14 \mathrm{~T} 15 \mathrm{~T} 6+\mathrm{Y} 14 \mathrm{~T} 18 \mathrm{~T} 6+\mathrm{Y} 14 \mathrm{~T} 20 \mathrm{~T} 6=1$

$\mathrm{Y} 14 \mathrm{~T} 7+\mathrm{Y} 14 \mathrm{~T} 6 \mathrm{~T} 7+\mathrm{Y} 14 \mathrm{~T} 10 \mathrm{~T} 7+\mathrm{Y} 14 \mathrm{~T} 18 \mathrm{~T} 7+$

$\mathrm{Y} 14 \mathrm{~T} 19 \mathrm{~T} 7=1$

$\mathrm{Y} 14 \mathrm{~T} 8+\mathrm{Y} 14 \mathrm{~T} 15 \mathrm{~T} 8+\mathrm{Y} 14 \mathrm{~T} 18 \mathrm{~T} 8+\mathrm{Y} 14 \mathrm{~T} 22 \mathrm{~T} 8=1$

$\mathrm{Y} 14 \mathrm{~T} 9+\mathrm{Y} 14 \mathrm{~T} 3 \mathrm{~T} 9+\mathrm{Y} 14 \mathrm{~T} 6 \mathrm{~T} 9+\mathrm{Y} 14 \mathrm{~T} 11 \mathrm{~T} 9+$

Y14T15T9+Y14T16T9+Y14T18T9+Y14T22T9+

Y14T23T9=1

Y14T10+Y14T6T10+Y14T7T10+Y14T1810+

Y14T1910=1

$\mathrm{Y} 14 \mathrm{~T} 11+\mathrm{Y} 14 \mathrm{~T} 3 \mathrm{~T} 11+\mathrm{Y} 14 \mathrm{~T} 6 \mathrm{~T} 11+\mathrm{Y} 14 \mathrm{~T} 9 \mathrm{~T} 11+$

$\mathrm{Y} 14 \mathrm{~T} 1511+\mathrm{Y} 14 \mathrm{~T} 1611+\mathrm{Y} 14 \mathrm{~T} 1811+\mathrm{Y} 14 \mathrm{~T} 2211=1$

$Y 14 T 13+Y 14 T 1813=1$

Y14T15+Y14T2T15+Y14T6T15+Y14T8T15+

Y14T9T15+Y14T1115+Y14T1615+Y14T1815+

$\mathrm{Y} 14 \mathrm{~T} 2015+\mathrm{Y} 14 \mathrm{~T} 2215=1$

Y14T16+Y14T9T16+Y14T1116+Y14T1516+

$Y 14 T 1816+Y 14 T 2316=1$

Y14T18+Y14T1T18+Y14T2T18+Y14T3T18+ Y14T5T18+Y14T6T18+Y14T7T18+Y14T8T18+ $Y 14 T 9 T 18+Y 14 T 1018+Y 14 T 1118+Y 14 T 1318+$ Y14T1518+Y14T1618+Y14T1918+Y14T2018+ $Y 14 T 2218+Y 14 T 2318+Y 14 T 2418+Y 14 T 2518=1$ Y14T19+Y14T2T19+Y14T7T19+Y14T1019+ $\mathrm{Y} 14 \mathrm{~T} 1819=1$

Y14T20+Y14T2T20+Y14T6T20+Y14T1520+ $Y 14 T 1820+Y 14 T 2220=1$

$\mathrm{Y} 14 \mathrm{~T} 22+\mathrm{Y} 14 \mathrm{~T} 2 \mathrm{~T} 22+\mathrm{Y} 14 \mathrm{~T} 8 \mathrm{~T} 22+\mathrm{Y} 14 \mathrm{~T} 9 \mathrm{~T} 22+$ Y14T1122+Y14T1522+Y14T1822+Y14T2022+ $\mathrm{Y} 14 \mathrm{~T} 2322=1$

Y14T23+Y14T3T23+Y14T9T23+Y14T1623+

$\mathrm{Y} 14 \mathrm{~T} 1823+\mathrm{Y} 14 \mathrm{~T} 2223=1$

$\mathrm{Y} 14 \mathrm{~T} 24+\mathrm{Y} 14 \mathrm{~T} 5 \mathrm{~T} 24+\mathrm{Y} 14 \mathrm{~T} 1824=1$

$\mathrm{Y} 14 \mathrm{~T} 25+\mathrm{Y} 14 \mathrm{~T} 1825=1$

$\mathrm{Y} 15 \mathrm{~T} 2+\mathrm{Y} 15 \mathrm{~T} 6 \mathrm{~T} 2+\mathrm{Y} 15 \mathrm{~T} 14 \mathrm{~T} 2+\mathrm{Y} 15 \mathrm{~T} 18 \mathrm{~T} 2+$

$Y 15 T 20 T 2+Y 15 T 22 T 2=1$

Y15T6+Y15T2T6+Y15T9T6+Y15T11T6+

$\mathrm{Y} 15 \mathrm{~T} 14 \mathrm{~T} 6+\mathrm{Y} 15 \mathrm{~T} 18 \mathrm{~T} 6+\mathrm{Y} 15 \mathrm{~T} 20 \mathrm{~T} 6=1$

$\mathrm{Y} 15 \mathrm{~T} 8+\mathrm{Y} 15 \mathrm{~T} 14 \mathrm{~T} 8+\mathrm{Y} 15 \mathrm{~T} 18 \mathrm{~T} 8+\mathrm{Y} 15 \mathrm{~T} 22 \mathrm{~T} 8=1$

Y15T9+Y15T6T9+Y15T11T9+Y15T14T9+

$\mathrm{Y} 15 \mathrm{~T} 16 \mathrm{~T} 9+\mathrm{Y} 15 \mathrm{~T} 18 \mathrm{~T} 9+\mathrm{Y} 15 \mathrm{~T} 22 \mathrm{~T} 9=1$

$\mathrm{Y} 15 \mathrm{~T} 11+\mathrm{Y} 15 \mathrm{~T} 6 \mathrm{~T} 11+\mathrm{Y} 15 \mathrm{~T} 9 \mathrm{~T} 11+\mathrm{Y} 15 \mathrm{~T} 1411+$

$Y 15 T 1611+Y 15 T 1811+Y 15 T 2211=1$

Y15T14+Y15T2T14+Y15T6T14+Y15T8T14+

Y15T9T14+Y15T1114+Y15T1614+Y15T1814+

Y15T2014+Y15T2214=1

$\mathrm{Y} 15 \mathrm{~T} 16+\mathrm{Y} 15 \mathrm{~T} 9 \mathrm{~T} 16+\mathrm{Y} 15 \mathrm{~T} 1116+\mathrm{Y} 15 \mathrm{~T} 1416+$

$Y 15 \mathrm{~T} 1816=1$

$\mathrm{Y} 15 \mathrm{~T} 18+\mathrm{Y} 15 \mathrm{~T} 2 \mathrm{~T} 18+\mathrm{Y} 15 \mathrm{~T} 6 \mathrm{~T} 18+\mathrm{Y} 15 \mathrm{~T} 8 \mathrm{~T} 18+$

Y15T9T18+Y15T1118+Y15T1418+Y15T1618+
$Y 15 T 2018+Y 15 T 2218=1$

Y15T20+Y15T2T20+Y15T6T20+Y15T1420+

$Y 15 T 1820+Y 15 T 2220=1$

Y15T22+Y15T2T22+Y15T8T22+Y15T9T22+

$\mathrm{Y} 15 \mathrm{~T} 1122+\mathrm{Y} 15 \mathrm{~T} 1422+\mathrm{Y} 15 \mathrm{~T} 1822+\mathrm{Y} 15 \mathrm{~T} 2022=1$

Y16T9+Y16T11T9+Y16T14T9+Y16T15T9+

$\mathrm{Y} 16 \mathrm{~T} 18 \mathrm{~T} 9+\mathrm{Y} 16 \mathrm{~T} 23 \mathrm{~T} 9=1$

$\mathrm{Y} 16 \mathrm{~T} 11+\mathrm{Y} 16 \mathrm{~T} 9 \mathrm{~T} 11+\mathrm{Y} 16 \mathrm{~T} 1411+\mathrm{Y} 16 \mathrm{~T} 1511+$

$\mathrm{Y} 16 \mathrm{~T} 1811=1$

Y16T14+Y16T9T14+Y16T1114+Y16T1514+

$\mathrm{Y} 16 \mathrm{~T} 1814+\mathrm{Y} 16 \mathrm{~T} 2314=1$

Y16T15+Y16T9T15+Y16T1115+Y16T1415+

$Y 16 \mathrm{~T} 1815=1$

$\mathrm{Y} 16 \mathrm{~T} 18+\mathrm{Y} 16 \mathrm{~T} 9 \mathrm{~T} 18+\mathrm{Y} 16 \mathrm{~T} 1118+\mathrm{Y} 16 \mathrm{~T} 1418+$

$\mathrm{Y} 16 \mathrm{~T} 1518+\mathrm{Y} 16 \mathrm{~T} 2318=1$

Y16T23+Y16T9T23+Y16T1423+Y16T1823=1

$\mathrm{Y} 17 \mathrm{~T} 4+\mathrm{Y} 17 \mathrm{~T} 18 \mathrm{~T} 4=1$

$\mathrm{Y} 17 \mathrm{~T} 11+\mathrm{Y} 17 \mathrm{~T} 1811+\mathrm{Y} 17 \mathrm{~T} 2111=1$

$\mathrm{Y} 17 \mathrm{~T} 18+\mathrm{Y} 17 \mathrm{~T} 4 \mathrm{~T} 18+\mathrm{Y} 17 \mathrm{~T} 1118+\mathrm{Y} 17 \mathrm{~T} 2118=1$

$\mathrm{Y} 17 \mathrm{~T} 21+\mathrm{Y} 17 \mathrm{~T} 1121+\mathrm{Y} 17 \mathrm{~T} 1821=1$

$\mathrm{Y} 18 \mathrm{~T} 1+\mathrm{Y} 18 \mathrm{~T} 5 \mathrm{~T} 1+\mathrm{Y} 18 \mathrm{~T} 6 \mathrm{~T} 1+\mathrm{Y} 18 \mathrm{~T} 14 \mathrm{~T} 1=1$

$\mathrm{Y} 18 \mathrm{~T} 2+\mathrm{Y} 18 \mathrm{~T} 6 \mathrm{~T} 2+\mathrm{Y} 18 \mathrm{~T} 14 \mathrm{~T} 2+\mathrm{Y} 18 \mathrm{~T} 15 \mathrm{~T} 2+$

$\mathrm{Y} 18 \mathrm{~T} 19 \mathrm{~T} 2+\mathrm{Y} 18 \mathrm{~T} 20 \mathrm{~T} 2+\mathrm{Y} 18 \mathrm{~T} 22 \mathrm{~T} 2=1$

$\mathrm{Y} 18 \mathrm{~T} 3+\mathrm{Y} 18 \mathrm{~T} 9 \mathrm{~T} 3+\mathrm{Y} 18 \mathrm{~T} 11 \mathrm{~T} 3+\mathrm{Y} 18 \mathrm{~T} 14 \mathrm{~T} 3+$

$\mathrm{Y} 18 \mathrm{~T} 21 \mathrm{~T} 3+\mathrm{Y} 18 \mathrm{~T} 23 \mathrm{~T} 3=1$

$\mathrm{Y} 18 \mathrm{~T} 4+\mathrm{Y} 18 \mathrm{~T} 17 \mathrm{~T} 4=1$

Y18T5+Y18T1T5+Y18T6T5+Y18T14T5+

Y18T24T5=1

$\mathrm{Y} 18 \mathrm{~T} 6+\mathrm{Y} 18 \mathrm{~T} 1 \mathrm{~T} 6+\mathrm{Y} 18 \mathrm{~T} 2 \mathrm{~T} 6+\mathrm{Y} 18 \mathrm{~T} 5 \mathrm{~T} 6+$

Y18T7T6+Y18T9T6+Y18T10T6+Y18T11T6+

$\mathrm{Y} 18 \mathrm{~T} 14 \mathrm{~T} 6+\mathrm{Y} 18 \mathrm{~T} 15 \mathrm{~T} 6+\mathrm{Y} 18 \mathrm{~T} 20 \mathrm{~T} 6=1$

$\mathrm{Y} 18 \mathrm{~T} 7+\mathrm{Y} 18 \mathrm{~T} 6 \mathrm{~T} 7+\mathrm{Y} 18 \mathrm{~T} 10 \mathrm{~T} 7+\mathrm{Y} 18 \mathrm{~T} 14 \mathrm{~T} 7+$

$\mathrm{Y} 18 \mathrm{~T} 19 \mathrm{~T} 7=1$

$\mathrm{Y} 18 \mathrm{~T} 8+\mathrm{Y} 18 \mathrm{~T} 14 \mathrm{~T} 8+\mathrm{Y} 18 \mathrm{~T} 15 \mathrm{~T} 8+\mathrm{Y} 18 \mathrm{~T} 22 \mathrm{~T} 8=1$

Y18T9+Y18T3T9+Y18T6T9+Y18T11T9+

Y18T14T9+Y18T15T9+Y18T16T9+Y18T22T9+

Y18T23T9=1

$\mathrm{Y} 18 \mathrm{~T} 10+\mathrm{Y} 18 \mathrm{~T} 6 \mathrm{~T} 10+\mathrm{Y} 18 \mathrm{~T} 7 \mathrm{~T} 10+\mathrm{Y} 18 \mathrm{~T} 1410+$

$Y 18 \mathrm{~T} 1910=1$

Y18T11+Y18T3T11+Y18T6T11+Y18T9T11+

$\mathrm{Y} 18 \mathrm{~T} 1411+\mathrm{Y} 18 \mathrm{~T} 1511+\mathrm{Y} 18 \mathrm{~T} 1611+\mathrm{Y} 18 \mathrm{~T} 1711+$

$Y 18 T 2111+Y 18 T 2211=1$

$Y 18 T 12=1$

$\mathrm{Y} 18 \mathrm{~T} 13+\mathrm{Y} 18 \mathrm{~T} 1413=1$

Y18T14+Y18T1T14+Y18T2T14+Y18T3T14+ Y18T5T14+Y18T6T14+Y18T7T14+Y18T8T14+ Y18T9T14+Y18T1014+Y18T1114+Y18T1314+ Y18T1514+Y18T1614+Y18T1914+Y18T2014+ $\mathrm{Y} 18 \mathrm{~T} 2214+\mathrm{Y} 18 \mathrm{~T} 2314+\mathrm{Y} 18 \mathrm{~T} 2414+\mathrm{Y} 18 \mathrm{~T} 2514=1$ Y18T15+Y18T2T15+Y18T6T15+Y18T8T15+ Y18T9T15+Y18T1115+Y18T1415+Y18T1615+ $\mathrm{Y} 18 \mathrm{~T} 2015+\mathrm{Y} 18 \mathrm{~T} 2215=1$

Y18T16+Y18T9T16+Y18T1116+Y18T1416+ $\mathrm{Y} 18 \mathrm{~T} 1516+\mathrm{Y} 18 \mathrm{~T} 2316=1$

$\mathrm{Y} 18 \mathrm{~T} 17+\mathrm{Y} 18 \mathrm{~T} 4 \mathrm{~T} 17+\mathrm{Y} 18 \mathrm{~T} 1117+\mathrm{Y} 18 \mathrm{~T} 2117=1$ Y18T19+Y18T2T19+Y18T7T19+Y18T1019+ $\mathrm{Y} 18 \mathrm{~T} 1419=1$

$\mathrm{Y} 18 \mathrm{~T} 20+\mathrm{Y} 18 \mathrm{~T} 2 \mathrm{~T} 20+\mathrm{Y} 18 \mathrm{~T} 6 \mathrm{~T} 20+\mathrm{Y} 18 \mathrm{~T} 1420+$ $\mathrm{Y} 18 \mathrm{~T} 1520+\mathrm{Y} 18 \mathrm{~T} 2220=1$

$\mathrm{Y} 18 \mathrm{~T} 21+\mathrm{Y} 18 \mathrm{~T} 3 \mathrm{~T} 21+\mathrm{Y} 18 \mathrm{~T} 1121+\mathrm{Y} 18 \mathrm{~T} 1721+$ $\mathrm{Y} 18 \mathrm{~T} 2221=1$

$\mathrm{Y} 18 \mathrm{~T} 22+\mathrm{Y} 18 \mathrm{~T} 2 \mathrm{~T} 22+\mathrm{Y} 18 \mathrm{~T} 8 \mathrm{~T} 22+\mathrm{Y} 18 \mathrm{~T} 9 \mathrm{~T} 22+$ $\mathrm{Y} 18 \mathrm{~T} 1122+\mathrm{Y} 18 \mathrm{~T} 1422+\mathrm{Y} 18 \mathrm{~T} 1522+\mathrm{Y} 18 \mathrm{~T} 2022+$

$\mathrm{Y} 18 \mathrm{~T} 2122+\mathrm{Y} 18 \mathrm{~T} 2322=1$

Y18T23+Y18T3T23+Y18T9T23+Y18T1423+ 


\section{APPENDIX B: PROBLEM FORMULATION FILE EFFICIENT PASSENGERS NETWORK}

$\mathrm{Y} 18 \mathrm{~T} 1623+\mathrm{Y} 18 \mathrm{~T} 2223=1$

$\mathrm{Y} 18 \mathrm{~T} 24+\mathrm{Y} 18 \mathrm{~T} 5 \mathrm{~T} 24+\mathrm{Y} 18 \mathrm{~T} 1424=1$

$Y 18 T 25+Y 18 T 1425=1$

$\mathrm{Y} 19 \mathrm{~T} 2+\mathrm{Y} 19 \mathrm{~T} 14 \mathrm{~T} 2+\mathrm{Y} 19 \mathrm{~T} 18 \mathrm{~T} 2=1$

$\mathrm{Y} 19 \mathrm{~T} 7+\mathrm{Y} 19 \mathrm{~T} 10 \mathrm{~T} 7+\mathrm{Y} 19 \mathrm{~T} 14 \mathrm{~T} 7+\mathrm{Y} 19 \mathrm{~T} 18 \mathrm{~T} 7=1$

$\mathrm{Y} 19 \mathrm{~T} 10+\mathrm{Y} 19 \mathrm{~T} 7 \mathrm{~T} 10+\mathrm{Y} 19 \mathrm{~T} 1410+\mathrm{Y} 19 \mathrm{~T} 1810=1$ Y19T14+Y19T2T14+Y19T7T14+Y19T1014+ Y19T1814=1

Y19T18+Y19T2T18+Y19T7T18+Y19T1018+ $Y 19 T 1418=1$

Y20T2+Y20T6T2+Y20T14T2+Y20T15T2+

$\mathrm{Y} 20 \mathrm{~T} 18 \mathrm{~T} 2+\mathrm{Y} 20 \mathrm{~T} 22 \mathrm{~T} 2=1$

Y20T6+Y20T2T6+Y20T14T6+Y20T15T6+

$\mathrm{Y} 20 \mathrm{~T} 18 \mathrm{~T} 6=1$

Y20T14+Y20T2T14+Y20T6T14+Y20T1514+ $\mathrm{Y} 20 \mathrm{~T} 1814+\mathrm{Y} 20 \mathrm{~T} 2214=1$

Y20T15+Y20T2T15+Y20T6T15+Y20T1415+

$\mathrm{Y} 20 \mathrm{~T} 1815+\mathrm{Y} 20 \mathrm{~T} 2215=1$

Y20T18+Y20T2T18+Y20T6T18+Y20T1418+

$\mathrm{Y} 20 \mathrm{~T} 1518+\mathrm{Y} 20 \mathrm{~T} 2218=1$

$\mathrm{Y} 20 \mathrm{~T} 22+\mathrm{Y} 20 \mathrm{~T} 2 \mathrm{~T} 22+\mathrm{Y} 20 \mathrm{~T} 1422+\mathrm{Y} 20 \mathrm{~T} 1522+$

$\mathrm{Y} 20 \mathrm{~T} 1822=1$

$\mathrm{Y} 21 \mathrm{~T} 3+\mathrm{Y} 21 \mathrm{~T} 11 \mathrm{~T} 3+\mathrm{Y} 21 \mathrm{~T} 18 \mathrm{~T} 3=1$

Y21T11+Y21T3T11+Y21T1711+Y21T1811+

$\mathrm{Y} 21 \mathrm{~T} 2211=1$

$\mathrm{Y} 21 \mathrm{~T} 17+\mathrm{Y} 21 \mathrm{~T} 1117+\mathrm{Y} 21 \mathrm{~T} 1817=1$

$\mathrm{Y} 21 \mathrm{~T} 18+\mathrm{Y} 21 \mathrm{~T} 3 \mathrm{~T} 18+\mathrm{Y} 21 \mathrm{~T} 1118+\mathrm{Y} 21 \mathrm{~T} 1718+$

$Y 21 T 2218=1$

$\mathrm{Y} 21 \mathrm{~T} 22+\mathrm{Y} 21 \mathrm{~T} 1122+\mathrm{Y} 21 \mathrm{~T} 1822=1$

$\mathrm{Y} 22 \mathrm{~T} 2+\mathrm{Y} 22 \mathrm{~T} 14 \mathrm{~T} 2+\mathrm{Y} 22 \mathrm{~T} 15 \mathrm{~T} 2+\mathrm{Y} 22 \mathrm{~T} 18 \mathrm{~T} 2+$

$\mathrm{Y} 22 \mathrm{~T} 20 \mathrm{~T} 2=1$

$\mathrm{Y} 22 \mathrm{~T} 8+\mathrm{Y} 22 \mathrm{~T} 14 \mathrm{~T} 8+\mathrm{Y} 22 \mathrm{~T} 15 \mathrm{~T} 8+\mathrm{Y} 22 \mathrm{~T} 18 \mathrm{~T} 8=1$

$\mathrm{Y} 22 \mathrm{~T} 9+\mathrm{Y} 22 \mathrm{~T} 11 \mathrm{~T} 9+\mathrm{Y} 22 \mathrm{~T} 14 \mathrm{~T} 9+\mathrm{Y} 22 \mathrm{~T} 15 \mathrm{~T} 9+$

$\mathrm{Y} 22 \mathrm{~T} 18 \mathrm{~T} 9+\mathrm{Y} 22 \mathrm{~T} 23 \mathrm{~T} 9=1$

$\mathrm{Y} 22 \mathrm{~T} 11+\mathrm{Y} 22 \mathrm{~T} 9 \mathrm{~T} 11+\mathrm{Y} 22 \mathrm{~T} 1411+\mathrm{Y} 22 \mathrm{~T} 1511+$

$\mathrm{Y} 22 \mathrm{~T} 1811+\mathrm{Y} 22 \mathrm{~T} 2111=1$

Y22T14+Y22T2T14+Y22T8T14+Y22T9T14+ Y22T1114+Y22T1514+Y22T1814+Y22T2014+ $\mathrm{Y} 22 \mathrm{~T} 2314=1$

Y22T15+Y22T2T15+Y22T8T15+Y22T9T15+ $\mathrm{Y} 22 \mathrm{~T} 1115+\mathrm{Y} 22 \mathrm{~T} 1415+\mathrm{Y} 22 \mathrm{~T} 1815+\mathrm{Y} 22 \mathrm{~T} 2015=1$ $\mathrm{Y} 22 \mathrm{~T} 18+\mathrm{Y} 22 \mathrm{~T} 2 \mathrm{~T} 18+\mathrm{Y} 22 \mathrm{~T} 8 \mathrm{~T} 18+\mathrm{Y} 22 \mathrm{~T} 9 \mathrm{~T} 18+$ $\mathrm{Y} 22 \mathrm{~T} 1118+\mathrm{Y} 22 \mathrm{~T} 1418+\mathrm{Y} 22 \mathrm{~T} 1518+\mathrm{Y} 22 \mathrm{~T} 2018+$ $\mathrm{Y} 22 \mathrm{~T} 2118+\mathrm{Y} 22 \mathrm{~T} 2318=1$

$\mathrm{Y} 22 \mathrm{~T} 20+\mathrm{Y} 22 \mathrm{~T} 2 \mathrm{~T} 20+\mathrm{Y} 22 \mathrm{~T} 1420+\mathrm{Y} 22 \mathrm{~T} 1520+$ $\mathrm{Y} 22 \mathrm{~T} 1820=1$

$\mathrm{Y} 22 \mathrm{~T} 21+\mathrm{Y} 22 \mathrm{~T} 1121+\mathrm{Y} 22 \mathrm{~T} 1821=1$ $\mathrm{Y} 22 \mathrm{~T} 23+\mathrm{Y} 22 \mathrm{~T} 9 \mathrm{~T} 23+\mathrm{Y} 22 \mathrm{~T} 1423+\mathrm{Y} 22 \mathrm{~T} 1823=1$ $\mathrm{Y} 23 \mathrm{~T} 3+\mathrm{Y} 23 \mathrm{~T} 9 \mathrm{~T} 3+\mathrm{Y} 23 \mathrm{~T} 14 \mathrm{~T} 3+\mathrm{Y} 23 \mathrm{~T} 18 \mathrm{~T} 3=1$ Y23T9+Y23T3T9+Y23T14T9+Y23T16T9+ $\mathrm{Y} 23 \mathrm{~T} 18 \mathrm{~T} 9+\mathrm{Y} 23 \mathrm{~T} 22 \mathrm{~T} 9=1$

Y23T14+Y23T3T14+Y23T9T14+Y23T1614+ Y23T1814+Y23T2214=1

$\mathrm{Y} 23 \mathrm{~T} 16+\mathrm{Y} 23 \mathrm{~T} 9 \mathrm{~T} 16+\mathrm{Y} 23 \mathrm{~T} 1416+\mathrm{Y} 23 \mathrm{~T} 1816=1$ Y23T18+Y23T3T18+Y23T9T18+Y23T1418+

$\mathrm{Y} 23 \mathrm{~T} 1618+\mathrm{Y} 23 \mathrm{~T} 2218=1$

Y23T22+Y23T9T22+Y23T1422+Y23T1822=1

Y24T5+Y24T14T5+Y24T18T5=1

Y24T14+Y24T5T14+Y24T1814=1

Y24T18+Y24T5T18+Y24T1418=1

Y25T14+Y25T1814=1

Y25T18+Y25T1418=1

END

LEAVE 


\section{APPENDIX B: PROBLEM FORMULATION FILE EFFICIENT COMBINED NETWORK}

MIN

+4874.72Y1T5+2056.61Y1T6T5+794.49Y1T14T5+

738.08Y1T18T5+1616.94Y1T6+1395.84Y1T5T6+ 971.09Y1T14T6+663.50Y1T18T6+554.15Y1T14+ 515.35Y1T5T14+1371.85Y1T6T14+771.89Y1T18T14+ 447.71Y1T18+483.29Y1T5T18+1027.99Y1T6T18+ 695.74Y1T14T18+766.85Y2T6+748.59Y2T14T6+ 1068.31Y2T15T6+596.89Y2T18T6+1395.25Y2T20T6+ 310.75Y2T14+983.16Y2T6T14+829.14Y2T15T14+ 749.61Y2T18T14+589.36Y2T19T14+436.39Y2T20T14+ 1123.54Y2T22T14+3599.74Y2T15+1300.85Y2T6T15+ 544.50Y2T14T15+767.57Y2T18T15+1459.28Y2T20T15+ 2971.38Y2T22T15+381.97Y2T18+710.62Y2T6T18+ 658.20Y2T14T18+884.93Y2T15T18+523.36Y2T19T18+ 581.32Y2T20T18+1147.98Y2T22T18+927.55Y2T19+ 1004.71Y2T14T19+976.43Y2T18T19+660.45Y2T20+ 1718.61Y2T6T20+850.15Y2T14T20+1543.26Y2T15T20+ 772.46Y2T18T20+1841.17Y2T22T20+4614.64Y2T22+ 657.57Y2T14T22+2491.20Y2T15T22+858.17Y2T18T22+ 1867.15Y2T20T22+1989.63Y3T9+1534.79Y3T11T9+ 981.55Y3T14T9+675.03Y3T18T9+1782.87Y3T23T9+ 1041.80Y3T11+1307.05Y3T9T11+917.96Y3T14T11+ 772.44Y3T18T11+1226.90Y3T21 T11+725.79Y3T14+ 977.21Y3T9T14+767.27Y3T11T14+870.58Y3T18T14+ 1049.46Y3T23T14+500.46Y3T18+567.75Y3T9T18+ 604.36Y3T11T18+817.71Y3T14T18+777.13Y3T21T18+ $650.94 \mathrm{Y} 323 \mathrm{~T} 18+1049.83 \mathrm{Y} 3 \mathrm{~T} 21+1524.87 \mathrm{Y} 311 \mathrm{~T} 21+$ 1421.83Y3 T18T21+1667.43Y3T23+1975.11Y3T9T23+ 1408.08Y3T14T23+995.73Y3T18T23+5759.66Y4T17+ 909.13Y4T18T17+430.94Y4T18+824.72Y4T17T18+ 4874.72Y5T1+2236.05Y5T6T1+783.41Y5T14T1+ 791.86Y5T18T1+1313.09Y5T6+1934.70Y5T1 T6+ 809.34Y5T14T6+581.47Y5T18T6+384.72Y5T14+ 523.69Y5T1T14+1048.85Y5T6T14+692.19Y5T18T14+ 633.13Y5T24T14+370.11Y5T18+512.45Y5T1T18+ 719.18Y5T6T18+640.17Y5T14T18+507.39Y5T24T18+ 2281.70Y5T24+1369.24Y5T14T24+800.65Y5T18T24+ $1616.94 \mathrm{Y} 6 \mathrm{~T} 1+1286.31 \mathrm{Y} 6 \mathrm{~T} 5 \mathrm{~T} 1+969.04 \mathrm{Y} 6 \mathrm{~T} 14 \mathrm{~T} 1+$ $701.06 \mathrm{Y} 6 \mathrm{~T} 18 \mathrm{~T} 1+778.14 \mathrm{Y} 6 \mathrm{~T} 2+750.01 \mathrm{Y} 6 \mathrm{~T} 14 \mathrm{~T} 2+$ 1128.51Y6T15T2+645.98Y6T18T2+1341.68Y6T20T2+ 1124.35Y6T5+1778.90Y6T1 T5+847.48Y6T14T5+ 585.75Y6T18T5+1047.75Y6T7+1027.01Y6T10T7+ 857.63Y6T14T7+609.95Y6T18T7+634.85Y6T9+ 947.19Y6T11 T9+869.85Y6T14T9+1351.21Y6T15T9+ 463.34Y6T18T9+748.47Y6 $10+824.33 \mathrm{Y} 6 \mathrm{~T} 7 \mathrm{~T} 10+$ 762.83Y6T14T10+492.54Y6T18T10+1398.21Y6T11+ 1407.59Y6T9T1 1+951.49 Y6T14T11+1648.30Y6T15T11+ 526.41Y6T18T11+486.95Y6T14+957.49Y6T1T14+ 758.30Y6T2T14+602.10Y6T5T14+673.77Y6T7T14+ 604.59Y6T9T14+702.23Y6T10T14+582.99Y6T11 T14+ 616.93Y6T15T14+590.93Y6T18T14+1188.94Y6T20T14+ 722.82Y6T15+1305.98Y6T2T15+938.32Y6T9T15+ 845.55Y6T11T15+720.19Y6T14T15+605.81Y6T18T15+ 1481.58Y6T20 T15+208.76Y6T18+1027.93Y6T1T18+ 817.06Y6T2T18+622.64Y6T5T18+659.99Y6T7T18+ 441.77Y6T9T18+715.82Y6T10T18+558.17Y6T11T18+ 834.35Y6T14T18+669.08Y6T15T18+1296.24Y6T20T18+ 1206.54Y6T20+900.08Y6T2T20+924.35Y6T14T20+ 1148.63Y6T15T20+613.46Y6T18T20+1152.96Y7T6+ 1077.73Y7T10 T6+840.00Y7T14T6+596.53Y7T18T6+ 1388.15Y7T10+1632.81Y7T6T10+674.75Y7T14T10+ 665.76Y7T18T10+1166.00Y7T19T10+404.55Y7T14+ 1058.31Y7T6T14+568.75Y7T10T14+721.90Y7T18T14+ 645.91Y7T19T14+378.51Y7T18+751.25Y7T6T18+ 585.81Y7T10T18+705.92Y7T14T18+559.03Y7T19T18+
1038.35Y7T19+1309.18Y7T10T19+1091.59Y7T14T19+ 980.03Y7T18T19+804.66Y8T14+911.94Y8T15T14+ 1323.75Y8T18T14+880.66Y8T22T14+1834.76Y8T15+ 973.22Y8T14T15+2052.15Y8T18T15+1280.41Y8T22T15+ 2639.48Y8T18+1118.38Y8T14T18+1588.40Y8T15T18+ 1332.83Y8T22T18+1136.39Y8T22+996.12Y8T14T22+ 1398.81Y8T15T22+1780.46Y8T18T22+1785.95Y9T3+ 1533.30Y9T11T3+1022.02Y9T14T3+596.74Y9T18T3+ 1631.06Y9T23T3+735.42Y9T6+1019.95Y9T11 T6+ 872.67Y9T14T6+1262.04Y9T15T6+470.00Y9T18T6+ $3910.96 \mathrm{Y} 9 \mathrm{~T} 11+1969.43 \mathrm{Y} 9 \mathrm{~T} 3 \mathrm{~T} 11+1757.97 \mathrm{Y} 9 \mathrm{~T} 6 \mathrm{~T} 11+$ 835.86Y9T14T11+2395.36Y9T15T11+2426.63Y9T16T11+ $528.84 \mathrm{Y} 9 \mathrm{~T} 18 \mathrm{~T} 11+3304.82 \mathrm{Y} 9 \mathrm{~T} 22 \mathrm{~T} 11+448.55 \mathrm{Y} 9 \mathrm{~T} 14+$ 669.01Y9T3T14+885.35Y9T6T14+533.34Y9T11T14+ 640.55Y9T15T14+841.51Y9T16 T14+588.58Y9T18T14+ 627.77Y9T22T14+809.43Y9T23T14+1376.31Y9T15+ 1118.98Y9T6T15+1251.11Y9T11T15+686.49Y9T14T15+ 1282.94Y9T16 T15+623.10Y9T18T15+1027.93Y9T22T15+ 1769.71Y9T16+1643.67Y9T11T16+1283.30Y9T14T16+ $1827.64 Y 9 \mathrm{~T} 15 \mathrm{~T} 16+600.08 \mathrm{Y} 9 \mathrm{~T} 18 \mathrm{~T} 16+1618.34 \mathrm{Y} 9 \mathrm{~T} 23 \mathrm{~T} 16+$ 256.57Y9T18+716.10Y9T3T18+611.19Y9T6T18+ 475.54Y9T11 T18+747.74Y9T14T18+666.64Y9T15T18+ 686.21Y9T16T18+676.93Y9T22T18+656.73Y9T23T18+ 1334.54Y9T22+1961.07Y9T11T22+731.06Y9T14T22+ 1468.47Y9T15T22+651.36Y9T18T22+1312.63Y9T23T22+ $1709.86 \mathrm{Y} 9 \mathrm{~T} 23+1700.03 \mathrm{Y} 9 \mathrm{~T} 3 \mathrm{~T} 23+1344.86 \mathrm{Y} 9 \mathrm{~T} 14 \mathrm{~T} 23+$ 1710.35Y9T16 $23+756.03 \mathrm{Y} 9 \mathrm{~T} 18 \mathrm{~T} 23+1353.02 \mathrm{Y} 9 \mathrm{~T} 22 \mathrm{~T} 23+$ 718.23Y10T6+1012.49Y10T7T6+730.30Y10T14T6+ $554.54 \mathrm{Y} 10 \mathrm{~T} 18 \mathrm{~T} 6+1388.15 \mathrm{Y} 10 \mathrm{~T} 7+1383.89 \mathrm{Y} 10 \mathrm{~T} 6 \mathrm{~T} 7+$ $682.88 \mathrm{Y} 10 \mathrm{~T} 14 \mathrm{~T} 7+732.95 \mathrm{Y} 10 \mathrm{~T} 18 \mathrm{~T} 7+1299.65 \mathrm{Y} 10 \mathrm{~T} 19 \mathrm{~T} 7+$ 301.13Y10T14+876.72Y10T6T14+497.65Y10T7T14+ 694.64Y10T1814+627.91Y10T1914+339.14Y10T18+ 623.41Y10T6T18+508.89Y10T7T18+653.48Y10T1418+ $556.14 \mathrm{Y} 10 \mathrm{~T} 1918+945.49 \mathrm{Y} 10 \mathrm{~T} 19+1125.83 \mathrm{Y} 10 \mathrm{~T} 7 \mathrm{~T} 19+$ 1006.38Y10T1419+918.68Y10T1819+979.21Y11T3+ 1249.88Y11T9T3+959.40Y11T14T3+703.15Y11T18T3+ 1255.10Y11T21T3+1529.26Y11T6+1516.78Y11T9T6+ $980.36 \mathrm{Y} 11 \mathrm{~T} 14 \mathrm{~T} 6+1662.23 \mathrm{Y} 11 \mathrm{~T} 15 \mathrm{~T} 6+540.44 \mathrm{Y} 11 \mathrm{~T} 18 \mathrm{~T} 6+$ 2840.96Y11T9+1988.04Y11T3T9+1743.15Y11T6T9+ 844.59Y11T14T9+2607.87Y11T15T9+1959.64Y11T16T9+ $530.42 \mathrm{Y} 11 \mathrm{~T} 18 \mathrm{~T} 9+3228.03 \mathrm{Y} 11 \mathrm{~T} 22 \mathrm{~T} 9+555.55 \mathrm{Y} 11 \mathrm{~T} 14+$ 738.07Y11T3T14+1013.98Y11T6T14+682.47Y11T9T14+ 714.44Y11T1514+919.00Y11T1614+687.92Y11T1814+ 938.79Y11T2214+2289.41Y11T15+1821.06Y11T6T15+ $1820.68 Y 11 \mathrm{~T} 9 \mathrm{~T} 15+772.67 \mathrm{Y} 11 \mathrm{~T} 1415+1787.08 \mathrm{Y} 11 \mathrm{~T} 1615+$ $668.90 \mathrm{Y} 11 \mathrm{~T} 1815+2312.42 \mathrm{Y} 11 \mathrm{~T} 2215+1172.80 \mathrm{Y} 11 \mathrm{~T} 16+$ 1427.51Y11T9T16+1209.66Y11T1416+1655.80Y11T1516+ 723.08Y11T1816+1056.53Y11T17+838.17Y11T1817+ 1717.61Y11T2117+320.28Y11T18+627.52Y11T3T18+ 621.38Y11T6T18+371.63Y11T9T18+684.10Y11T1418+ $580.22 \mathrm{Y} 11 \mathrm{~T} 1518+631.18 \mathrm{Y} 11 \mathrm{~T} 1618+583.59 \mathrm{Y} 11 \mathrm{~T} 1718+$ 766.90Y11T2118+679.27Y11T2218+4117.89Y11T21+ 1304.39Y11T3T21+3247.03Y11T1721+1429.92Y11T1821+ 4053.97Y11 T2221+3329.10Y11T22+1706.60Y11T9T22+ 843.15Y11T1422+1826.67Y11T1522+745.31Y11T1822+ 2336.46Y11T2122+554.52Y12T18+1177.63Y13T14+ 771.93Y13T1814+432.88Y13T18+986.91Y13T1418+ 538.84Y14T1+498.05Y14T5T1+1383.77Y14T6T1+ 817.32Y14T18T1+289.04Y14T2+917.39Y14T6T2+ 830.11Y14T15T2+776.02Y14T18T2+613.06Y14T19T2+ 436.17Y14T20T2+1124.67Y14T22T2+713.99Y14T3+ $884.69 \mathrm{Y} 14 \mathrm{~T} 9 \mathrm{~T} 3+677.13 \mathrm{Y} 14 \mathrm{~T} 11 \mathrm{~T} 3+780.69 \mathrm{Y} 14 \mathrm{~T} 18 \mathrm{~T} 3+$ 978.74Y14T23T3+380.58Y14T5+507.50Y14T1T5+ 929.02Y14T6T5+686.76Y14T18T5+627.05Y14T24T5+ 455.82Y14T6+962.30Y14T1T6+758.44Y14T2T6+ 


\section{APPENDIX B: PROBLEM FORMULATION FILE EFFICIENT COMBINED NETWORK}

638.64Y14T5T6+748.34Y14T7T6+650.08Y14T9T6+ 649.30Y14T10T6+591.40Y14T11T6+528.59Y14T15T6+ 581.51Y14T18T6+1150.46Y14T20T6+370.79Y14T7+ 894.18Y14T6T7+548.19Y14T10T7+726.98Y14T18T7+ 685.00Y14T19T7+983.14Y14T8+916.92Y14T15T8+ 1267.28Y14T18T8+917.41Y14T22T8+396.34Y14T9+ 713.60Y14T3T9+765.63Y14T6T9+480.25Y14T11 T9+ 636.36Y14T15T9+755.90Y14T16 T9+585.99Y14T18T9+ 596.91Y14T22T9+766.37Y14T23T9+281.20Y14T10+ 867.42Y14T6T10+476.72Y14T7T10+621.43Y14T1810+ $570.53 \mathrm{Y} 14 \mathrm{~T} 1910+492.18 \mathrm{Y} 14 \mathrm{~T} 11+769.60 \mathrm{Y} 14 \mathrm{~T} 3 \mathrm{~T} 11+$ 914.89Y14T6T11+632.15Y14T9T11+660.41Y14T1511+ 999.68Y14T1611+665.82Y14T1811+925.25Y14T2211+ 1316.49Y14T13+770.38Y14T1813+225.72Y14T15+ 879.95Y14T2T15+830.99Y14T6T15+565.68Y14T8T15+ 563.67Y14T9T15+449.87Y14T1115+771.93Y14T1615+ 703.59Y14T1815+557.41Y14T2015+531.79Y14T2215+ 1031.75Y14T16+983.66Y14T9T16+844.24Y14T1116+ 893.44Y14T1516+785.67Y14T1816+1133.03Y14T2316+ 350.41Y14T18+463.84Y14T1T18+614.27Y14T2T18+ 727.17Y14T3T18+487.59Y14T5T18+628.97Y14T6T18+ 572.38Y14T7T18+722.91Y14T8T18+424.28Y14T9T18+ $565.05 \mathrm{Y} 14 \mathrm{~T} 1018+451.55 \mathrm{Y} 14 \mathrm{~T} 1118+600.27 \mathrm{Y} 14 \mathrm{~T} 1318+$ 477.52Y14T1518+797.70Y14T1618+665.65Y14T1918+ 502.55Y14T2018+588.64Y14T2218+724.46Y14T2318+ $532.26 \mathrm{Y} 14 \mathrm{~T} 2418+463.06 \mathrm{Y} 14 \mathrm{~T} 2518+841.14 \mathrm{Y} 14 \mathrm{~T} 19+$ 756.29Y14T2T19+819.07Y14T7T19+775.70Y14T1019+ $882.82 \mathrm{Y} 14 \mathrm{~T} 1819+1056.70 \mathrm{Y} 14 \mathrm{~T} 20+860.07 \mathrm{Y} 14 \mathrm{~T} 2 \mathrm{~T} 20+$ 1645.41Y14T6T20+1224.39Y14T1520+798.45Y14T1820+ 1395.63Y14T2220+353.13Y14T22+1095.49Y14T2T22+ $531.90 \mathrm{Y} 14 \mathrm{~T} 8 \mathrm{~T} 22+616.18 \mathrm{Y} 14 \mathrm{~T} 9 \mathrm{~T} 22+556.61 \mathrm{Y} 14 \mathrm{~T} 1122+$ 498.73Y14T1522+756.83Y14T1822+574.98Y14T2022+ 648.27Y14T2322+1125.44Y14T23+1074.14Y14T3T23+ 1078.49Y14T9T23+1302.98Y14T1623+891.55Y14T1823+ 877.43Y14T2223+1165.70Y14T24+1147.89Y14T5T24+ 829.74Y14T1824+217.79Y14T25+719.86Y14T1825+ 1675.93Y15T2+1129.05Y15T6T2+516.40Y15T14T2+ 762.62Y15T18T2+1333.10Y15T20T2+1814.13Y15T22T2+ 639.93Y15T6+1249.79Y15T2T6+1046.32Y15T9T6+ 838.42Y15T11 T6+668.24Y15T14T6+541.25Y15T18T6+ 1697.28Y15T20T6+1682.65Y15T8+1033.77Y15T14T8+ 1806.01Y15T18T8+1310.75Y15T22T8+1396.70Y15T9+ 912.88Y15T6T9+1172.15Y15T11T9+626.62Y15T14T9+ 1205.86Y15T16 T9+546.15Y15T18T9+997.50Y15T22T9+ 1911.52Y15T11+1615.83Y15T6T11+1882.69Y15T9T11+ $710.14 \mathrm{Y} 15 \mathrm{~T} 1411+1870.76 \mathrm{Y} 15 \mathrm{~T} 1611+611.96 \mathrm{Y} 15 \mathrm{~T} 1811+$ 2231.36Y15T2211+232.87Y15T14+896.72Y15T2T14+ 796.42Y15T6T14+541.48Y15T8T14+606.79Y15T9T14+ 461.35Y15T1114+771.85Y15T1614+657.05Y15T1814+ 736.97Y15T2014+543.22Y15T2214+1733.97Y15T16+ 1859.74Y15T9T16+1687.78Y15T1116+1170.45Y15T1416+ 729.31Y15T1816+318.81Y15T18+1004.90Y15T2T18+ 570.08Y15T6T18+686.65Y15T8T18+503.97Y15T9T18+ 486.57Y15T1118+586.72Y15T1418+642.69Y15T1618+ 891.30Y15T2018+611.59Y15T2218+1345.56Y15T20+ 1468.00Y15T2T20+1680.52Y15T6T20+1116.84Y15T1420+ 722.47Y15T1820+1375.61Y15T2220+644.96Y15T22+ 1803.81Y15T2T22+712.99Y15T8T22+1047.76Y15T9T22+ 938.84Y15T1122+567.62Y $15 \mathrm{~T} 1422+737.20 \mathrm{Y} 15 \mathrm{~T} 1822+$ 1384.93Y15T2022+1744.45Y16 T9+1863.81Y16T11T9+ 1332.06Y16T14T9+2026.10Y16 T15T9+660.29Y16 T18T9+ 1801.46Y16T23T9+1571.48Y16 T11+1433.61Y16T9T11+ 1239.72Y16T1411+1651.24Y16T1511+775.63Y16T1811+ 1148.10Y16T14+1031.80Y16T9T14+1090.46Y16T1114+ 994.38Y16T1514+853.64Y16T1814+1252.14Y16T2314+
2098.18Y16T15+1830.75Y16T9T15+2142.52Y16T1115+ 1272.59Y16T1415+830.19Y16T1815+495.69Y16T18+ $528.05 Y 16 \mathrm{~T} 9 \mathrm{~T} 18+785.59 \mathrm{Y} 16 \mathrm{~T} 1118+985.42 \mathrm{Y} 16 \mathrm{~T} 1418+$ 725.28Y16T1518+673.04Y16T2318+1702.33Y16T23+ 1853.09Y16T9T23+1758.59Y16T1423+966.79Y16T1823+ 11999.66Y17T4+1022.03Y17T18T4+1216.53Y17T11+ 970.13Y17T1811+1853.65Y17T2111+785.22Y17T18+ 969.96Y17T4T18+824.75Y17T1118+1344.47Y17T2118+ 4420.73Y17T21+2625.95Y17T1121+1598.39Y17T1821+ 483.56Y18T1+484.87Y18T5T1+1061.62Y18T6T1+ 699.24Y18T14T1+428.28Y18T2+704.45Y18T6T2+ 640.87Y18T14T2+948.10Y18T15T2+498.53Y18T19T2+ $596.59 \mathrm{Y} 18 \mathrm{~T} 20 \mathrm{~T} 2+1200.54 \mathrm{Y} 18 \mathrm{~T} 22 \mathrm{~T} 2+426.32 \mathrm{Y} 18 \mathrm{~T} 3+$ 536.44Y18T9T3+556.97Y18T11T3+820.78Y18T14T3+ 725.42Y18T21 T3+640.79Y18T23T3+346.35Y18T4+ 702.84Y18T17T4+368.65Y18T5+559.46Y18T1T5+ 683.81Y18T6T5+658.95Y18T14T5+511.44Y18T24T5+ 209.41Y18T6+1070.72Y18T1T6+886.59Y18T2T6+ 669.41Y18T5T6+765.33Y18T7T6+536.76Y18T9T6+ $629.09 \mathrm{Y} 18 \mathrm{~T} 10 \mathrm{~T} 6+618.10 \mathrm{Y} 18 \mathrm{~T} 11 \mathrm{~T} 6+807.08 \mathrm{Y} 18 \mathrm{~T} 14 \mathrm{~T} 6+$ $652.03 \mathrm{Y} 18 \mathrm{~T} 15 \mathrm{~T} 6+1319.15 \mathrm{Y} 18 \mathrm{~T} 20 \mathrm{~T} 6+401.29 \mathrm{Y} 18 \mathrm{~T} 7+$ 663.67Y18T6T7+516.06Y18T10T7+703.82Y18T14T7+ $534.29 \mathrm{Y} 18 \mathrm{~T} 19 \mathrm{~T} 7+2241.58 \mathrm{Y} 18 \mathrm{~T} 8+1195.26 \mathrm{Y} 18 \mathrm{~T} 14 \mathrm{~T} 8+$ 1559.54Y18T15T8+1394.43Y 18T22T8+246.27Y18T9+ $658.52 \mathrm{Y} 18 \mathrm{~T} 3 \mathrm{~T} 9+522.60 \mathrm{Y} 18 \mathrm{~T} 6 \mathrm{~T} 9+457.44 \mathrm{Y} 18 \mathrm{~T} 11 \mathrm{~T} 9+$ 707.62Y18T14T9+723.64Y18T15T9+602.01Y18T16T9+ $651.09 \mathrm{Y} 18 \mathrm{~T} 22 \mathrm{~T} 9+676.12 \mathrm{Y} 18 \mathrm{~T} 23 \mathrm{~T} 9+275.28 \mathrm{Y} 18 \mathrm{~T} 10+$ $650.04 \mathrm{Y} 18 \mathrm{~T} 6 \mathrm{~T} 10+510.94 \mathrm{Y} 18 \mathrm{~T} 7 \mathrm{~T} 10+640.79 \mathrm{Y} 18 \mathrm{~T} 1410+$ 461.58Y18T1910+310.30Y18T11+603.47Y18T3T11+ $575.94 \mathrm{Y} 18 \mathrm{~T} 6 \mathrm{~T} 11+381.44 \mathrm{Y} 18 \mathrm{~T} 9 \mathrm{~T} 11+649.40 \mathrm{Y} 18 \mathrm{~T} 1411+$ $593.94 \mathrm{Y} 18 \mathrm{~T} 1511+745.53 \mathrm{Y} 18 \mathrm{~T} 1611+551.44 \mathrm{Y} 18 \mathrm{~T} 1711+$ 713.08Y18T2111+700.99Y18T2211+547.62Y18T12+ 434.11Y18T13+1022.86Y18T1413+364.65Y18T14+ $510.22 \mathrm{Y} 18 \mathrm{~T} 1 \mathrm{~T} 14+682.51 \mathrm{Y} 18 \mathrm{~T} 2 \mathrm{~T} 14+667.72 \mathrm{Y} 18 \mathrm{~T} 3 \mathrm{~T} 14+$ 491.41Y18T5T14+667.82Y18T6T14+604.19Y18T7T14+ 645.63Y18T8T14+480.77Y18T9T14+529.24Y18T1014+ 494.33Y18T1114+603.00Y18T1314+541.60Y18T1514+ $809.01 \mathrm{Y} 18 \mathrm{~T} 1614+621.90 \mathrm{Y} 18 \mathrm{~T} 1914+531.83 \mathrm{Y} 18 \mathrm{~T} 2014+$ $629.58 \mathrm{Y} 18 \mathrm{~T} 2214+790.31 \mathrm{Y} 18 \mathrm{~T} 2314+512.76 \mathrm{Y} 18 \mathrm{~T} 2414+$ 506.77Y18T2514+387.33Y18T15+1056.60Y18T2T15+ $640.19 \mathrm{Y} 18 \mathrm{~T} 6 \mathrm{~T} 15+654.90 \mathrm{Y} 18 \mathrm{~T} 8 \mathrm{~T} 15+514.33 \mathrm{Y} 18 \mathrm{~T} 9 \mathrm{~T} 15+$ $517.48 \mathrm{Y} 18 \mathrm{~T} 1115+593.02 \mathrm{Y} 18 \mathrm{~T} 1415+660.53 \mathrm{Y} 18 \mathrm{~T} 1615+$ 738.51Y18T2015+646.08Y18T2215+439.87Y18T16+ 553.55Y18T9T16+619.92Y18T1116+932.14Y18T1416+ 726.22Y18T1516+649.93Y18T2316+624.36Y18T17+ 845.61Y18T4T17+706.46Y18T1117+1120.34Y18T2117+ 729.63Y18T19+968.35Y18T2T19+870.60Y18T7T19+ 821.23Y18T1019+1029.53Y18T1419+413.59Y18T20+ $678.87 \mathrm{Y} 18 \mathrm{~T} 2 \mathrm{~T} 20+1232.34 \mathrm{Y} 18 \mathrm{~T} 6 \mathrm{~T} 20+626.93 \mathrm{Y} 18 \mathrm{~T} 1420+$ $906.59 \mathrm{Y} 18 \mathrm{~T} 1520+773.00 \mathrm{Y} 18 \mathrm{~T} 2220+1170.55 \mathrm{Y} 18 \mathrm{~T} 21+$ 899.22Y18T3T21+1200.42Y18T1121+1366.28Y18T1721+ 1274.25Y18T2221+467.31Y18T22+1292.32Y18T2T22+ 616.68Y18T8T22+499.51Y18T9T22+627.14Y18T1122+ 701.79Y18T1422+661.17Y18T1522+775.78Y18T2022+ 708.52Y18T2122+602.08Y18 T2322+604.92Y18T23+ 760.32Y18T3T23+677.98Y18T9T23+976.04Y18T1423+ $760.12 \mathrm{Y} 18 \mathrm{~T} 1623+736.31 \mathrm{Y} 18 \mathrm{~T} 2223+501.69 \mathrm{Y} 18 \mathrm{~T} 24+$ $695.60 \mathrm{Y} 18 \mathrm{~T} 5 \mathrm{~T} 24+921.06 \mathrm{Y} 18 \mathrm{~T} 1424+658.76 \mathrm{Y} 18 \mathrm{~T} 25+$ $593.09 \mathrm{Y} 18 \mathrm{~T} 1425+683.49 \mathrm{Y} 19 \mathrm{~T} 2+916.61 \mathrm{Y} 19 \mathrm{~T} 14 \mathrm{~T} 2+$ 1047.14Y19T18T2+1387.30 Y19T7+1287.65Y19T10T7+ 1105.13Y19T14T7+1150.84Y19T18T7+942.97Y19T10+ 1111.32Y19T7T10+976.79Y19T1410+965.56Y19T1810+ 841.14Y19T14+767.48Y19T2T14+844.34Y19T7T14+ 810.38Y19T1014+974.39Y19T1814+988.40Y19T18+ 894.74Y19T2T18+906.37Y19T7T18+876.47Y19T1018+ 


\section{APPENDIX B: PROBLEM FORMULATION FILE EFFICIENT COMBINED NETWORK}

1045.52Y19T1418+594.99Y20T2+1667.91Y20T6T2+ $810.20 \mathrm{Y} 20 \mathrm{~T} 14 \mathrm{~T} 2+1217.53 \mathrm{Y} 20 \mathrm{~T} 15 \mathrm{~T} 2+813.56 \mathrm{Y} 20 \mathrm{~T} 18 \mathrm{~T} 2+$ 1673.85Y20T22T2+1206.54Y20T6+889.11Y20T2T6+ 915.18Y20T14T6+894.01Y20T15T6+599.30Y20T18T6+ $1490.29 \mathrm{Y} 20 \mathrm{~T} 14+870.68 \mathrm{Y} 20 \mathrm{~T} 2 \mathrm{~T} 14+1595.87 \mathrm{Y} 20 \mathrm{~T} 6 \mathrm{~T} 14+$ 1280.37Y20T1514+799.50Y20T1814+1699.89Y20T2214+ $1260.55 \mathrm{Y} 20 \mathrm{~T} 15+1699.42 \mathrm{Y} 20 \mathrm{~T} 2 \mathrm{~T} 15+1846.25 \mathrm{Y} 20 \mathrm{~T} 6 \mathrm{~T} 15+$ 1255.83Y20T1415+753.46Y20T1815+1530.91Y20T2215+ 417.81Y20T18+637.87Y20T2T18+1210.14Y20T6T18+ 629.36Y20T1418+662.94Y20T1518+735.93Y20T2218+ 1795.25Y20T22+1965.04Y20T2T22+1323.43Y20T1422+ 1197.71Y20T1522+825.73Y20T1822+1581.89Y21T3+ 1883.98Y21T11T3+1547.28Y21T18T3+3776.46Y21T11+ 1682.78Y21T3T11+2881.29Y21T1711+1530.04Y21T1811+ 4125.27Y21T2211+4060.73Y21T17+2602.62Y21T1117+ 1565.25Y21T1817+1301.07Y21T18+1308.68Y21T3T18+ 1256.86Y21T1118+1488.15Y21T1718+1338.79Y21T2218+ 4188.92Y21T22+4420.70Y21T1122+1670.41Y21T1822+ 2686.74Y22T2+649.41Y22T14T2+1577.88Y22T15T2+ 827.53Y22T18T2+1463.74Y22T20T2+1125.86Y22T8+ $1060.53 \mathrm{Y} 22 \mathrm{~T} 14 \mathrm{~T} 8+1314.55 \mathrm{Y} 22 \mathrm{~T} 15 \mathrm{~T} 8+1580.00 \mathrm{Y} 22 \mathrm{~T} 18 \mathrm{~T} 8+$ 1246.91Y22T9+1867.61Y22T11T9+697.95Y22T14T9+ 1448.56Y22T15 T9+590.27Y22T18T9+1292.98Y22T23T9+ 3092.26Y22T11+1693.83Y22T9T11+800.19Y22T1411+ 1600.36Y22T1511+681.14Y22T1811+2145.78Y22T2111+ 370.78Y22T14+1128.99Y22T2T14+527.36Y22T8T14+ 622.56Y22T9T14+585.19Y22T1114+505.65Y22T1514+ 735.03Y22T $1814+605.90 \mathrm{Y} 22 \mathrm{~T} 2014+665.04 \mathrm{Y} 22 \mathrm{~T} 2314+$ 603.06Y22T15+1830.82Y22T2T15+696.55Y22T8T15+ 937.34Y22T9T15+895.52Y22T11 15+578.62Y22T1415+ 766.97Y22T1815+1100.04Y22T2015+414.45Y22T18+ 1239.30Y22T2T18+696.53Y22T $8 \mathrm{~T} 18+471.45 \mathrm{Y} 22 \mathrm{~T} 9 \mathrm{~T} 18+$ $620.05 \mathrm{Y} 22 \mathrm{~T} 1118+696.31 \mathrm{Y} 22 \mathrm{~T} 1418+599.69 \mathrm{Y} 22 \mathrm{~T} 1518+$ 774.23Y22T2018+766.50Y22T2118+540.63Y22T2318+ 1378.60Y22T20+1763.33Y22T2T20+1180.41Y22T1420+ 1252.27Y22T1520+803.51Y22T1820+2917.25Y22T21+ 4140.07Y22T1121+1480.15Y22T1821+968.47Y22T23+ 1296.92Y22T9T23+1181.20Y22T1423+910.29Y22T1823+ $1310.06 \mathrm{Y} 23 \mathrm{~T} 3+1846.39 \mathrm{Y} 23 \mathrm{~T} 9 \mathrm{~T} 3+1397.46 \mathrm{Y} 23 \mathrm{~T} 14 \mathrm{~T} 3+$ 857.73Y23T18T3+1748.64Y23T9+1718.86Y23T3T9+ 1315.81Y23T14T9+1584.11Y23T16T9+701.01Y23T18T9+ 1317.73Y23T22T9+1125.44Y23T14+967.43Y23T3T14+ 1093.18Y23T9T14+1259.31Y23T1614+862.22Y23T1814+ 854.40Y23T2214+1282.33Y23T16+1838.84Y23T9T16+ 1658.74Y23T1416+846.87Y23T1816+534.37Y $23 \mathrm{~T} 18+$ 801.46Y23T3T18+645.74Y23T9T18+1000.70Y23T1418+ 770.13Y23T1618+721.38Y23T2218+952.09Y23T22+ 1305.04Y23T9T22+1164.67Y23T1422+893.09Y23T1822+ 2244.55Y24T5+1368.58Y24T14T5+794.28Y24T18T5+ 1120.18Y24T14+1147.18Y24T5T14+850.20Y24T1814+ 514.21Y24T18+694.47Y24T5T18+896.34Y24T1418+ 223.28Y25T14+709.08Y25T1814+680.09Y25T18+ $595.80 \mathrm{Y} 25 \mathrm{~T} 1418$ ST

Y1T6-Y1T6T5 > $=0$

Y6T5-Y1T6T5 $>=0$

Y1T14-Y1T14T5 $>=0$

Y14T5-Y1T14T5 $>=0$

Y1T18-Y1T18T5 $>=0$

Y18T5-Y1T18T5 $>=0$

Y1T5-Y1T5T6>=0

Y5T6-Y1T5T6 $>=0$

Y1T14-Y1T14T6 $>=0$

Y14T6-Y1T14T6 > $=0$

Y1T18-Y1T18T6 >=0
$\mathrm{Y} 18 \mathrm{~T} 6-\mathrm{Y} 1 \mathrm{~T} 18 \mathrm{~T} 6>=0$

Y1T5-Y1T5T14 > $=0$

Y5T14-Y1T5T14 >=0

Y1T6-Y1T6T14 > = 0

Y6T14-Y1T6T14 $>=0$

$Y 1 T 18-Y 1 T 18 T 14>=0$

Y18T14Y $1 \mathrm{~T} 18 \mathrm{~T} 14>=0$

Y1T5-Y1T5T18 > $=0$

Y5T18-Y1T5T18 > = 0

Y1T6-Y1T6T18 > $=0$

Y6T18-Y1T6T18 $>=0$

Y1T14-Y1T14T18 > 0

Y14T18-Y1T14T18 >=0

$\mathrm{Y} 2 \mathrm{~T} 14-\mathrm{Y} 2 \mathrm{~T} 14 \mathrm{~T} 6>=0$

Y14T6-Y2T14T6 >= 0

Y2T15-Y2T15T6 > $=0$

Y15T6-Y2T15T6 > $=0$

Y2T18-Y2T18T6 > $=0$

Y18T6-Y2T18T6 $>=0$

Y2T20-Y2T20T6 $>=0$

Y20T6-Y2T20T6 > $=0$

Y2T6-Y2T6T14 $>=0$

Y6T14-Y2T6T14 $>=0$ Y2T15-Y2T15T14 $>=0$ Y15T14-Y2T15T14 $>=0$ Y2T18-Y2T18T14 $>=0$ Y18T14-Y2T18T14 > $=0$ Y2T19-Y2T19T14 $>=0$ Y19T14-Y2T19T14 $>=0$ Y2T20-Y2T20T14 $>=0$ $\mathrm{Y} 20 \mathrm{~T} 14-\mathrm{Y} 2 \mathrm{~T} 20 \mathrm{~T} 14>=0$ Y2T22-Y2T22T14 > 0 $\mathrm{Y} 22 \mathrm{~T} 14-\mathrm{Y} 2 \mathrm{~T} 2 \mathrm{~T} 14>=0$ Y2T6-Y2T6T15 > $=0$ Y6T15-Y2T6T15 >= 0 Y2T14-Y2T14T15 > $=0$ $\mathrm{Y} 14 \mathrm{~T} 15-\mathrm{Y} 2 \mathrm{~T} 14 \mathrm{~T} 15>=0$ Y2T18-Y2T18T15 > $=0$ Y18T15-Y2T18T15 $>=0$ Y2T20-Y2T20T15 > $=0$ Y20T15-Y2T20T15 > $=0$ Y2T22-Y2T22T15 $>=0$ $\mathrm{Y} 22 \mathrm{~T} 15-\mathrm{Y} 2 \mathrm{~T} 22 \mathrm{~T} 15>=0$ Y2T6-Y2T6T18 $>=0$ Y6T18-Y2T6T18 >= 0 Y2T14-Y2T14T18 > $=0$ Y14T18-Y2T14T18 $>=0$ Y2T15-Y2T15T18 $>=0$ Y15T18-Y2T15T18 > $=0$ Y2T19-Y2T19T18 $>=0$ Y19T18-Y2T19T18 > = 0 Y2T20-Y2T20T18 > $=0$ Y20T18-Y2T20T18 $>=0$ Y2T22-Y2T22T18 > $=0$ Y22T18-Y2T22T18 > $=0$ Y2T14-Y2T14T19 > $=0$ Y14T19-Y2T14T19 > $=0$ Y2T18-Y2T18T19 > $=0$ Y18T19-Y2T18T19 $>=0$ Y2T6-Y2T6T20 > $=0$ Y6T20-Y2T6T20 > $=0$ Y2T14-Y2T14T20 $>=0$ Y14T20-Y2T14T20 > $>0$ Y2T15-Y2T15T20 > $=0$ Y15T20-Y2T15T20 > $=0$ 


\section{APPENDIX B: PROBLEM FORMULATION FILE EFFICIENT COMBINED NETWORK}

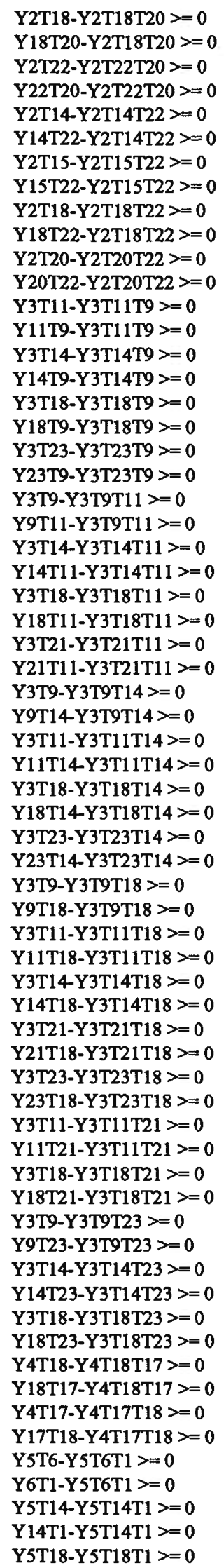

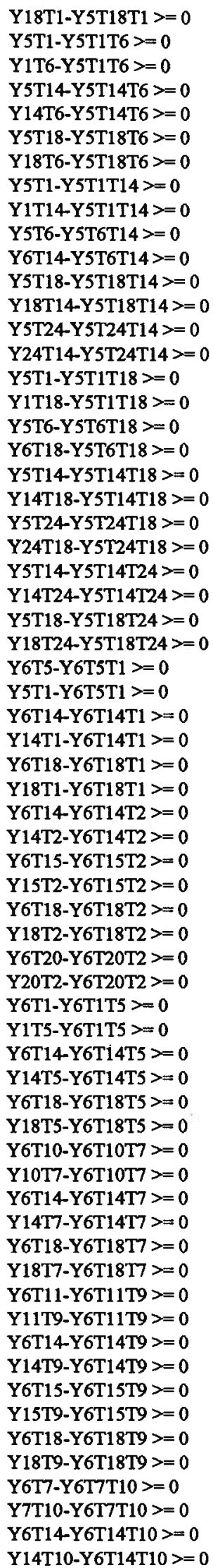




\section{APPENDIX B: PROBLEM FORMULATION FILE EFFICIENT COMBINED NETWORK}

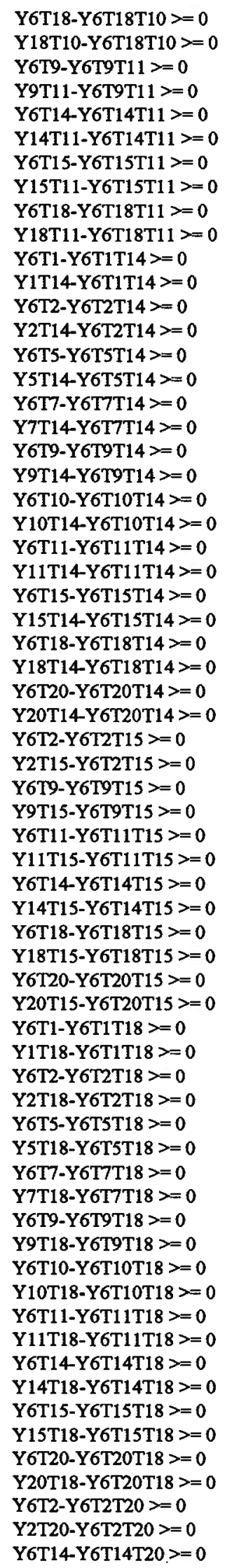

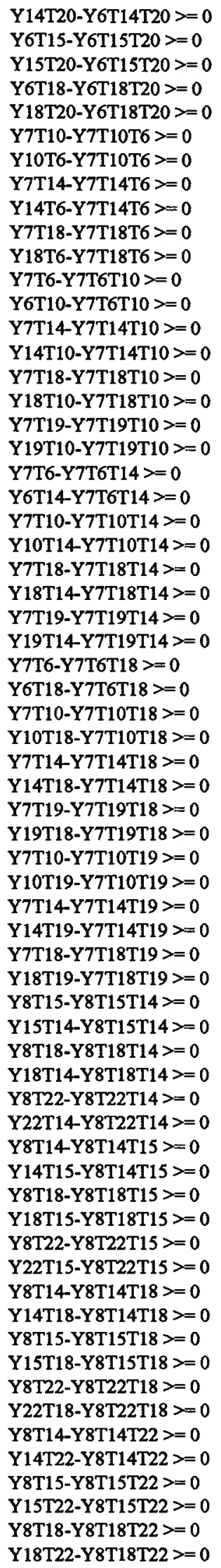




\section{APPENDIX B: PROBLEM FORMULATION FILE EFFICIENT COMBINED NETWORK}

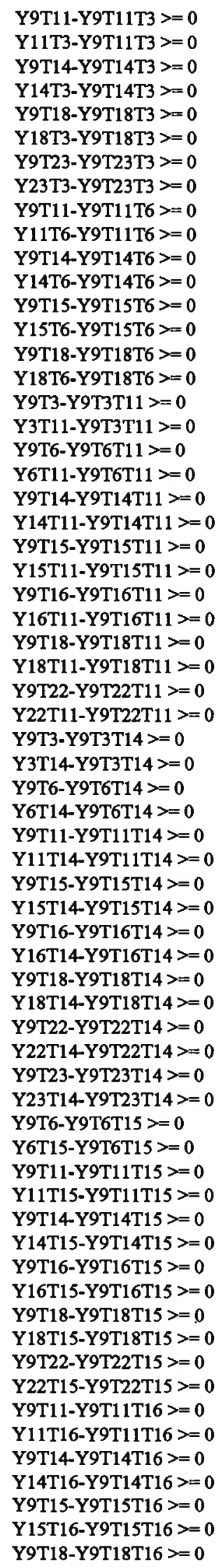

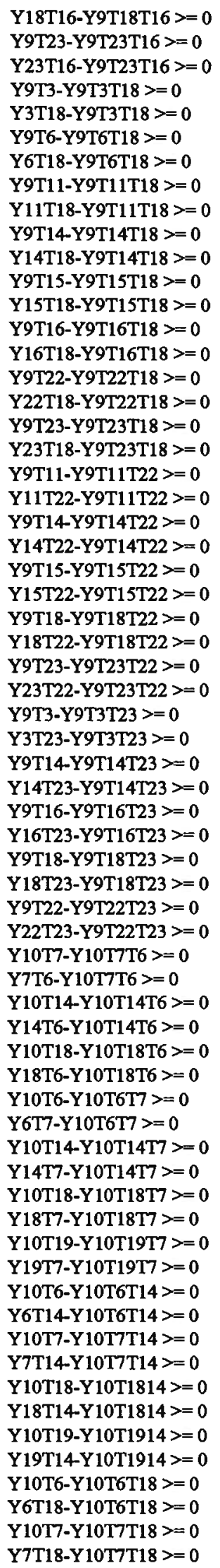




\section{APPENDIX B: PROBLEM FORMULATION FILE EFFICIENT COMBINED NETWORK}

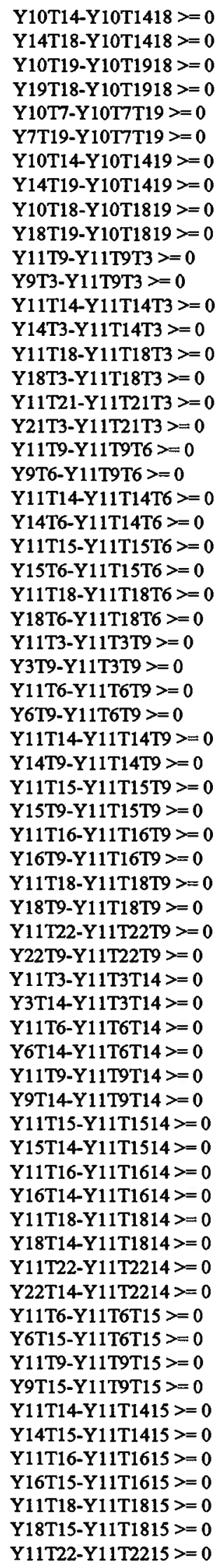

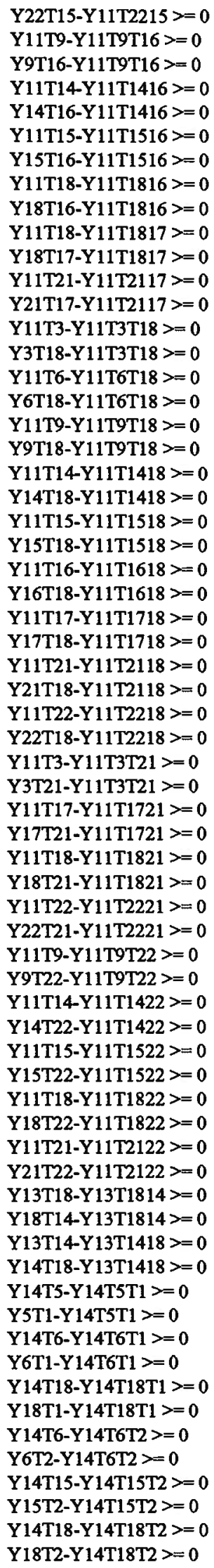




\section{APPENDIX B: PROBLEM FORMULATION FILE EFFICIENT COMBINED NETWORK}

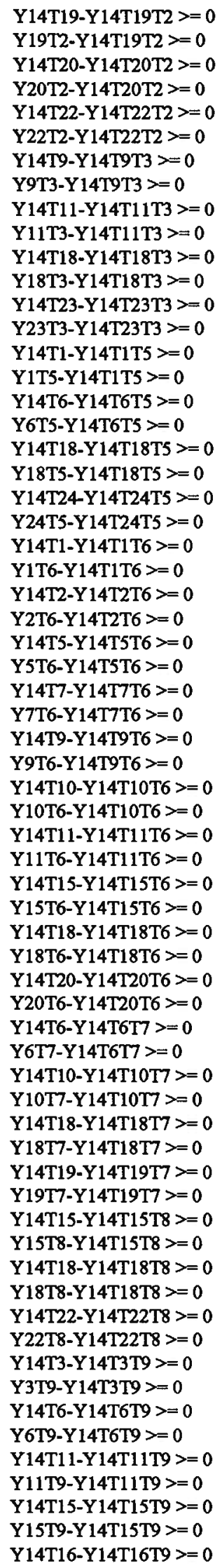

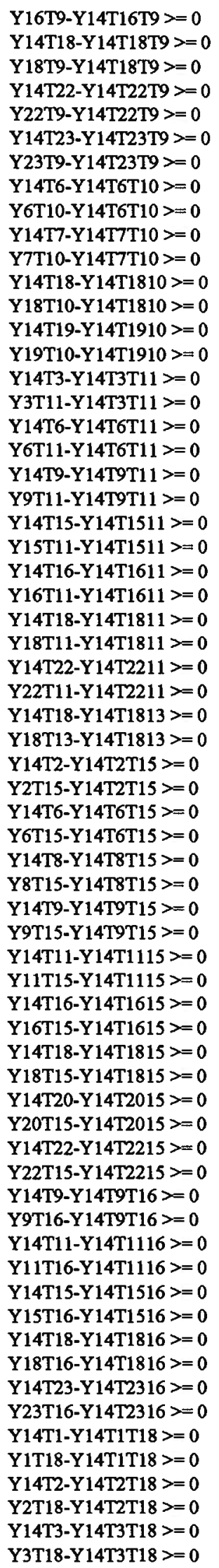




\section{APPENDIX B: PROBLEM FORMULATION FILE EFFICIENT COMBINED NETWORK}

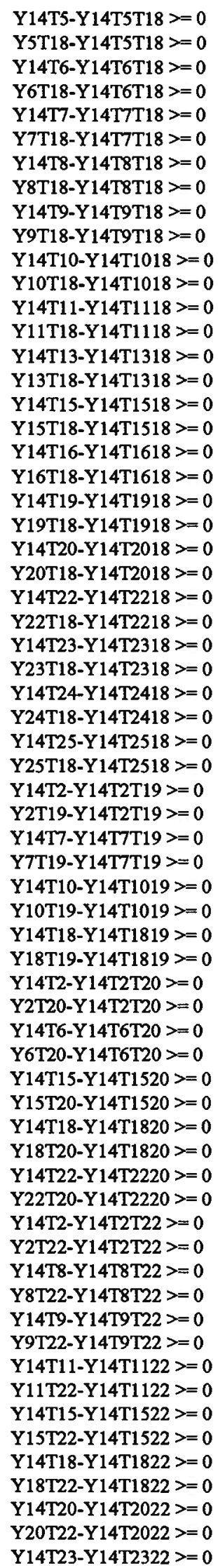

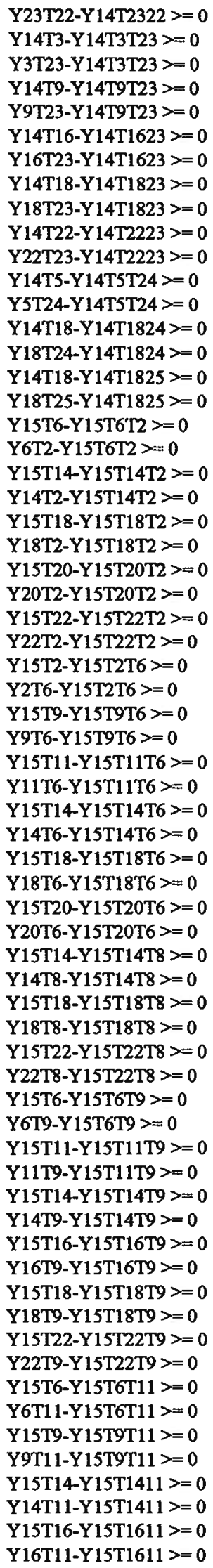




\section{APPENDIX B: PROBLEM FORMULATION FILE EFFICIENT COMBINED NETWORK}

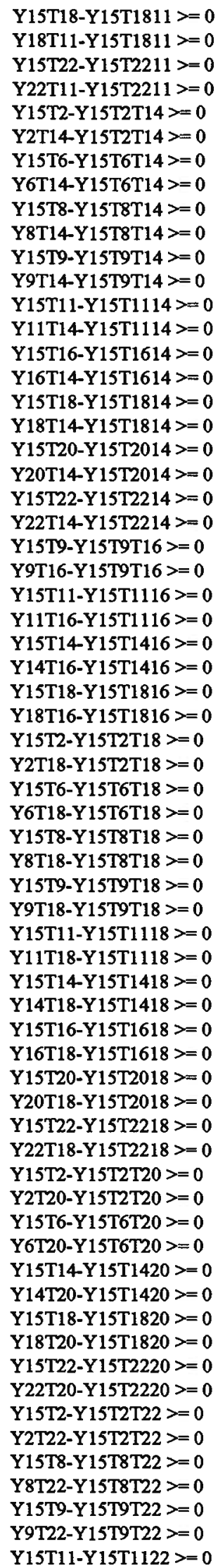

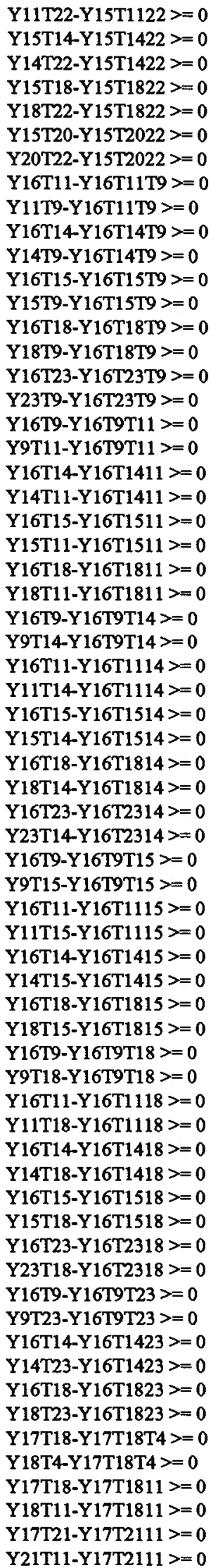




\title{
APPENDIX B: PROBLEM FORMULATION FILE EFFICIENT COMBINED NETWORK
}

\author{
Y17T4-Y17T4T18 > $=0$ \\ Y4T18-Y17T4T18 >=0 \\ Y17T11-Y17T1118>=0 \\ Y11T18-Y17T1118>=0 \\ Y17T21-Y17T2118>=0 \\ Y21T18-Y17T2118 $>=0$ \\ Y17T11-Y17T1121 >=0 \\ Y11T21-Y17T1121 >=0 \\ $\mathrm{Y} 17 \mathrm{~T} 18-\mathrm{Y} 17 \mathrm{~T} 1821>=0$ \\ Y18T21-Y17T1821 >=0 \\ Y18T5-Y18T5T1 $>=0$ \\ Y5T1-Y18T5T1 $>=0$ \\ Y18T6-Y18T6T1 >=0 \\ Y6T1-Y18T6T1 $>=0$ \\ $\mathrm{Y} 18 \mathrm{~T} 14-\mathrm{Y} 18 \mathrm{~T} 14 \mathrm{~T} 1>=0$ \\ $\mathrm{Y} 14 \mathrm{~T} 1-\mathrm{Y} 18 \mathrm{~T} 14 \mathrm{~T} 1>=0$ \\ Y18T6-Y18T6T2 >=0 \\ Y6T2-Y18T6T2 > $=0$ \\ $Y 18 \mathrm{~T} 14-Y 18 \mathrm{~T} 14 \mathrm{~T} 2>=0$ \\ Y14T2-Y18T14T2 >= 0 \\ $Y 18 T 15-Y 18 T 15 T 2>=0$ \\ Y15T2-Y18T15T2 > $=0$ \\ Y18T19-Y18T19T2 > $=0$ \\ Y19T2-Y18T19T2 > $=0$ \\ Y18T20-Y18T20T2 $>=0$ \\ $\mathrm{Y} 20 \mathrm{~T} 2-\mathrm{Y} 18 \mathrm{~T} 20 \mathrm{~T} 2>=0$ \\ Y18T22-Y18T22T2 $>=0$ \\ $\mathrm{Y} 22 \mathrm{~T} 2-\mathrm{Y} 18 \mathrm{~T} 22 \mathrm{~T} 2>=0$ \\ Y18T9-Y18T9T3 $>=0$ \\ Y9T3-Y18T9T3 > $=0$ \\ $Y 18 \mathrm{~T} 11-Y 18 \mathrm{~T} 11 \mathrm{~T} 3>=0$ \\ Y11T3-Y18T11T3 $>=0$ \\ Y18T14-Y18T14T3 $>=0$ \\ Y14T3-Y18T14T3 $>=0$ \\ $\mathrm{Y} 18 \mathrm{~T} 21-\mathrm{Y} 18 \mathrm{~T} 21 \mathrm{~T} 3>=0$ \\ Y21T3-Y18T21T3 $>=0$ \\ Y18T23-Y18T23T3 $>=0$ \\ Y23T3-Y18T23T3 $>=0$ \\ Y18T17-Y18T17T4 $>=0$ \\ Y17T4-Y18T17T4 $>=0$ \\ Y18T1-Y18T1T5 $>=0$ \\ Y1T5-Y18T1T5 $>=0$ \\ Y18T6-Y18T6T5 >= 0 \\ Y6T5-Y18T6T5 >= 0 \\ $\mathrm{Y} 18 \mathrm{~T} 14-\mathrm{Y} 18 \mathrm{~T} 14 \mathrm{~T} 5>=0$ \\ Y14T5-Y18T14T5 $>=0$ \\ Y18T24-Y18T24T5 > = 0 \\ Y24T5-Y18T24T5 > $=0$ \\ $\mathrm{Y} 18 \mathrm{~T} 1-\mathrm{Y} 18 \mathrm{~T} 1 \mathrm{~T} 6>=0$ \\ Y1T6-Y18T1T6 $>=0$ \\ Y18T2-Y18T2T6 > $=0$ \\ Y2T6-Y18T2T6 $>=0$ \\ Y18T5-Y18T5T6 $>=0$ \\ Y5T6-Y18T5T6 > $=0$ \\ Y18T7-Y18T7T6 > $=0$ \\ Y7T6-Y18T7T6 > $=0$ \\ Y18T9-Y18T9T6 $>=0$ \\ Y9T6-Y 18T9T6 > $=0$ \\ Y18T10-Y18T10T6 $>=0$ \\ Y10T6-Y18T10T6 >= 0 \\ Y18T11-Y18T11T6 $>=0$ \\ Y11T6-Y18T11T6 > $=0$ \\ Y18T14-Y18T14T6 $>=0$ \\ Y 14T6-Y18T14T6 > $=0$ \\ Y18T15-Y18T15T6 >=0
}

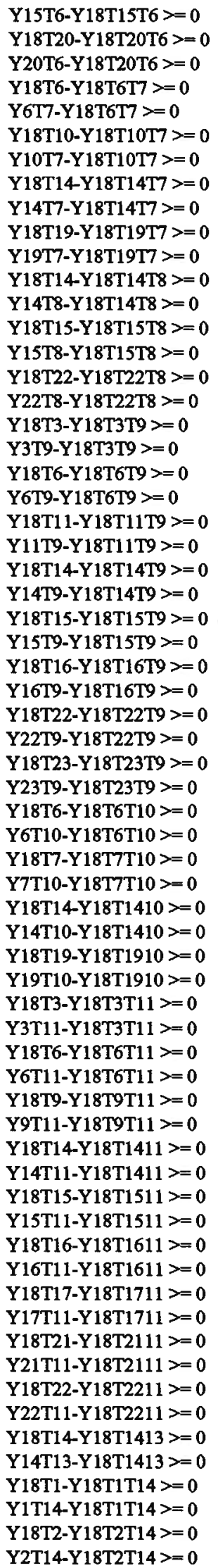




\section{APPENDIX B: PROBLEM FORMULATION FILE EFFICIENT COMBINED NETWORK}

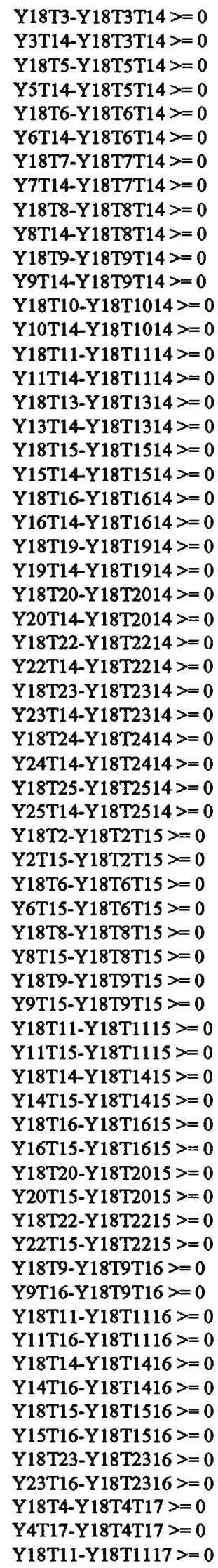

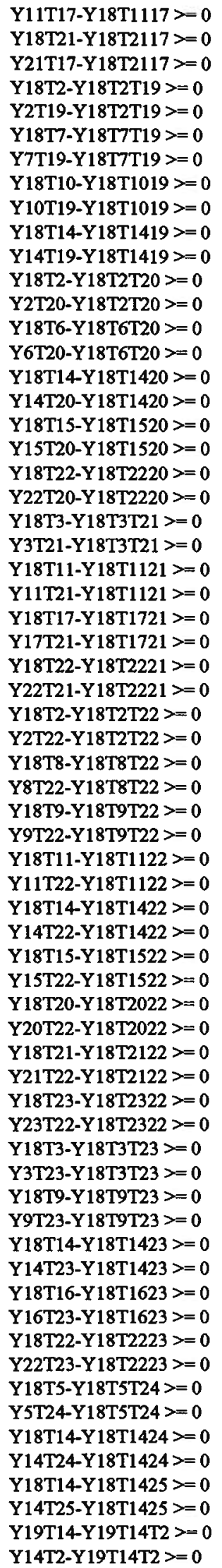




\section{APPENDIX B: PROBLEM FORMULATION FILE EFFICIENT COMBINED NETWORK}

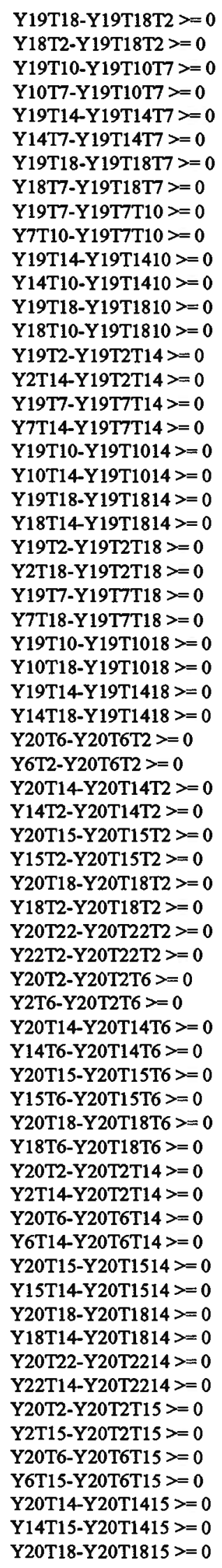

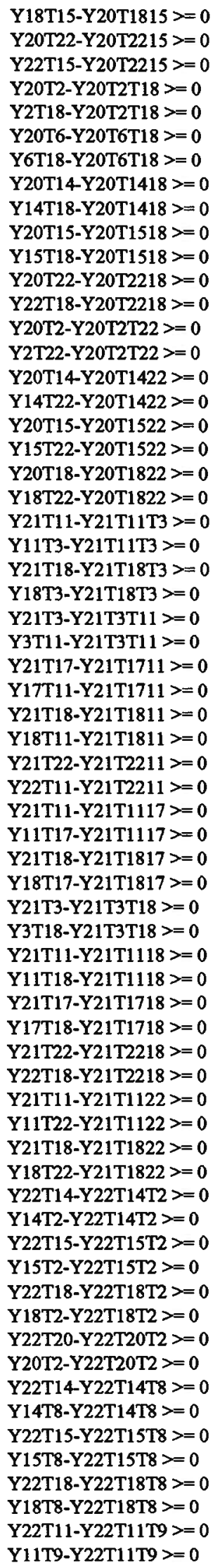




\section{APPENDIX B: PROBLEM FORMULATION FILE EFFICIENT COMBINED NETWORK}

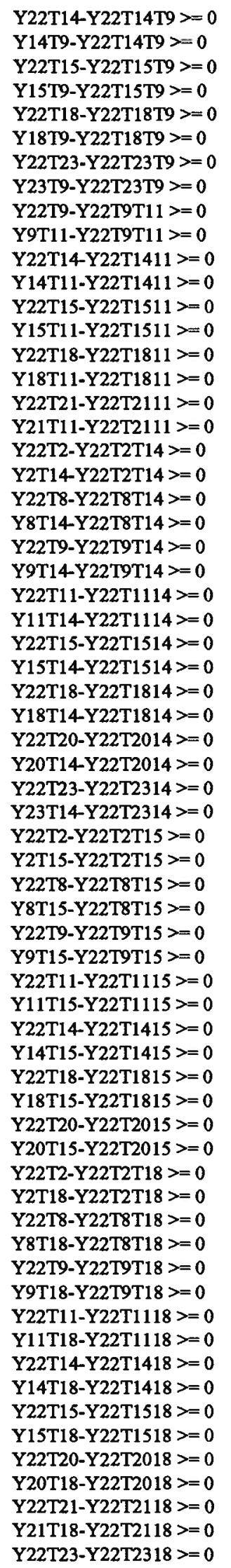

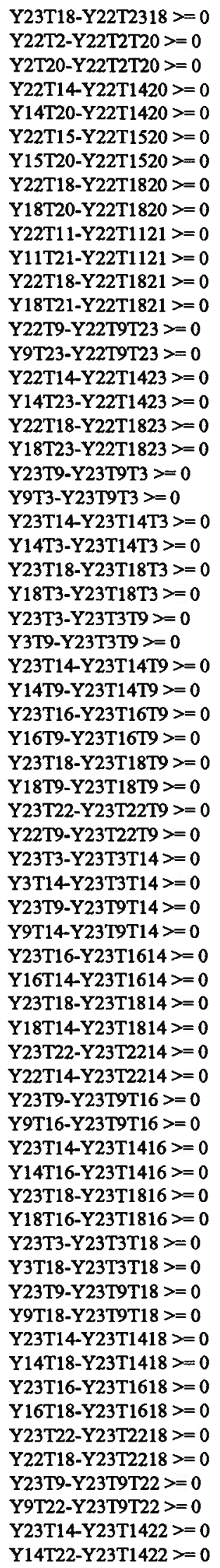




\section{APPENDIX B: PROBLEM FORMULATION FILE EFFICIENT COMBINED NETWORK}

Y23T18-Y23T1822 $>=0$ Y18T22-Y23T1822 > $=0$

Y24T14-Y24T14T5 > $=0$

$\mathrm{Y} 14 \mathrm{~T} 5-\mathrm{Y} 24 \mathrm{~T} 14 \mathrm{~T} 5>=0$

Y24T18-Y24T18T5 $>=0$

Y18T5-Y24T18T5 $>=0$

Y24T5-Y24T5T14 $>=0$

Y $5 \mathrm{~T} 14-\mathrm{Y} 24 \mathrm{~T} 5 \mathrm{~T} 14>=0$

Y24T18-Y24T1814 > $=0$

$\mathrm{Y} 18 \mathrm{~T} 14 \mathrm{Y} 24 \mathrm{~T} 1814>=0$

Y24T5-Y24T5T18 > $=0$

Y5T18-Y24T5T18 $>=0$

$\mathrm{Y} 24 \mathrm{~T} 14-\mathrm{Y} 24 \mathrm{~T} 1418>=0$

$\mathrm{Y} 14 \mathrm{~T} 18-\mathrm{Y} 24 \mathrm{~T} 1418>=0$

$\mathrm{Y} 25 \mathrm{~T} 18-\mathrm{Y} 25 \mathrm{~T} 1814>=0$

$\mathrm{Y} 18 \mathrm{~T} 14-\mathrm{Y} 25 \mathrm{~T} 1814>0$

$Y 25 T 14-Y 25 T 1418>=0$

$\mathrm{Y} 14 \mathrm{~T} 18-\mathrm{Y} 25 \mathrm{~T} 1418>=0$

$\mathrm{Y} 1 \mathrm{~T} 5+\mathrm{Y} 1 \mathrm{~T} 6 \mathrm{~T} 5+\mathrm{Y} 1 \mathrm{~T} 14 \mathrm{~T} 5+\mathrm{Y} 1 \mathrm{~T} 18 \mathrm{~T} 5=1$

Y1T6+Y1T5T6+Y1T14T6+Y1T18T6=1

Y1T14+Y1T5T14+Y1T6T14+Y1T18T14=1

$Y 1 T 18+Y 1 T 5 T 18+Y 1 T 6 T 18+Y 1 T 14 T 18=1$ Y2T6+Y2T14T6+Y2T15T6+Y2T18T6+

$\mathrm{Y} 2 \mathrm{~T} 20 \mathrm{~T} 6=1$

Y2T14+Y2T6T14+Y2T15T14+Y2T18T14+ Y2T19T14+Y2T20T14+Y2T22T14=1

Y2T15+Y2T6T15+Y2T14T15+Y2T18T15+ $\mathrm{Y} 2 \mathrm{~T} 20 \mathrm{~T} 15+\mathrm{Y} 2 \mathrm{~T} 22 \mathrm{~T} 15=1$

$\mathrm{Y} 2 \mathrm{~T} 18+\mathrm{Y} 2 \mathrm{~T} 6 \mathrm{~T} 18+\mathrm{Y} 2 \mathrm{~T} 14 \mathrm{~T} 18+\mathrm{Y} 2 \mathrm{~T} 15 \mathrm{~T} 18+$ $\mathrm{Y} 2 \mathrm{~T} 19 \mathrm{~T} 18+\mathrm{Y} 2 \mathrm{~T} 20 \mathrm{~T} 18+\mathrm{Y} 2 \mathrm{~T} 22 \mathrm{~T} 18=1$

$\mathrm{Y} 2 \mathrm{~T} 19+\mathrm{Y} 2 \mathrm{~T} 14 \mathrm{~T} 19+\mathrm{Y} 2 \mathrm{~T} 18 \mathrm{~T} 19=1$

$\mathrm{Y} 2 \mathrm{~T} 20+\mathrm{Y} 2 \mathrm{~T} 6 \mathrm{~T} 20+\mathrm{Y} 2 \mathrm{~T} 14 \mathrm{~T} 20+\mathrm{Y} 2 \mathrm{~T} 15 \mathrm{~T} 20+$ $\mathrm{Y} 2 \mathrm{~T} 18 \mathrm{~T} 20+\mathrm{Y} 2 \mathrm{~T} 22 \mathrm{~T} 20=1$

$\mathrm{Y} 2 \mathrm{~T} 22+\mathrm{Y} 2 \mathrm{~T} 14 \mathrm{~T} 22+\mathrm{Y} 2 \mathrm{~T} 15 \mathrm{~T} 22+\mathrm{Y} 2 \mathrm{~T} 18 \mathrm{~T} 22+$ Y2T20T22=1

Y3T9+Y3T11T9+Y3T14T9+Y3T18T9+

Y3T23T9=1

Y3T11+Y3T9T11+Y3T14T11+Y3T18T11+

$\mathrm{Y} 3 \mathrm{~T} 21 \mathrm{~T} 11=1$

Y3T14+Y3T9T14+Y3T11T14+Y3T18T14+

Y3T23T14=1

Y3T18+Y3T9T18+Y3T11T18+Y3T14T18+

Y3T21T18+Y3T23T18=1

$\mathrm{Y} 3 \mathrm{~T} 21+\mathrm{Y} 3 \mathrm{~T} 11 \mathrm{~T} 21+\mathrm{Y} 3 \mathrm{~T} 18 \mathrm{~T} 21=1$

Y3T23+Y3T9T23+Y3T14T23+Y3T18T23=1

$\mathrm{Y} 4 \mathrm{~T} 17+\mathrm{Y} 4 \mathrm{~T} 18 \mathrm{~T} 17=1$

$\mathrm{Y} 4 \mathrm{~T} 18+\mathrm{Y} 4 \mathrm{~T} 17 \mathrm{~T} 18=1$

$\mathrm{Y} 5 \mathrm{~T} 1+\mathrm{Y} 5 \mathrm{~T} 6 \mathrm{~T} 1+\mathrm{Y} 5 \mathrm{~T} 14 \mathrm{~T} 1+\mathrm{Y} 5 \mathrm{~T} 18 \mathrm{~T} 1=1$

Y5T6+Y5T1T6+Y5T14T6+Y5T18T6=1 Y5T14+Y5T1T14+Y5T6T14+Y5T18T14+ Y5T24T14=1 Y5T18+Y5T1T18+Y5T6T18+Y5T14T18+

$\mathrm{Y} 5 \mathrm{~T} 24 \mathrm{~T} 18=1$

Y5T24+Y5T14T24+Y5T18T24=1

$\mathrm{Y} 6 \mathrm{~T} 1+\mathrm{Y} 6 \mathrm{~T} 5 \mathrm{~T} 1+\mathrm{Y} 6 \mathrm{~T} 14 \mathrm{~T} 1+\mathrm{Y} 6 \mathrm{~T} 18 \mathrm{~T} 1=1$

$\mathrm{Y} 6 \mathrm{~T} 2+\mathrm{Y} 6 \mathrm{~T} 14 \mathrm{~T} 2+\mathrm{Y} 6 \mathrm{~T} 15 \mathrm{~T} 2+\mathrm{Y} 6 \mathrm{~T} 18 \mathrm{~T} 2+$

$\mathrm{Y} 6 \mathrm{~T} 20 \mathrm{~T} 2=1$

Y6T5+Y6T1T5+Y6T14T5+Y6T18T5=1 $\mathrm{Y} 6 \mathrm{~T} 7+\mathrm{Y} 6 \mathrm{~T} 10 \mathrm{~T} 7+\mathrm{Y} 6 \mathrm{~T} 14 \mathrm{~T} 7+\mathrm{Y} 6 \mathrm{~T} 18 \mathrm{~T} 7=1$ Y6T9+Y6T11T9+Y6T14T9+Y6T15T9+ $\mathrm{Y} 6 \mathrm{~T} 18 \mathrm{~T} 9=1$

Y6T10+Y6T7T10+Y6T14T10+Y6T18T10=1 Y $6 \mathrm{~T} 11+\mathrm{Y} 6 \mathrm{~T} 9 \mathrm{~T} 11+\mathrm{Y} 6 \mathrm{~T} 14 \mathrm{~T} 11+\mathrm{Y} 6 \mathrm{~T} 15 \mathrm{~T} 11+$ Y6T18T11=1

Y6T14+Y6T1T14+Y6T2T14+Y6T5T14+
Y6T7T14+Y6T9T14+Y6T10T14+Y6T11T14+

Y6T15T14+Y6T18T14+Y6T20T14=1

Y6T15+Y6T2T15+Y6T9T15+Y6T11T15+

Y6T14T15+Y6T18T15+Y6T20T15=1 $\mathrm{Y} 6 \mathrm{~T} 18+\mathrm{Y} 6 \mathrm{~T} 1 \mathrm{~T} 18+\mathrm{Y} 6 \mathrm{~T} 2 \mathrm{~T} 18+\mathrm{Y} 6 \mathrm{~T} 5 \mathrm{~T} 18+$ Y6T7T18+Y6T9T18+Y6T10T18+Y6T11T18+ $\mathrm{Y} 6 \mathrm{~T} 14 \mathrm{~T} 18+\mathrm{Y} 6 \mathrm{~T} 15 \mathrm{~T} 18+\mathrm{Y} 6 \mathrm{~T} 20 \mathrm{~T} 18=1$ Y6T20+Y6T2T20+Y6T14T20+Y6T15T20+ $\mathrm{Y} 6 \mathrm{~T} 18 \mathrm{~T} 20=1$ Y7T6+Y7T10T6+Y7T14T6+Y7T18T6=1 Y7T10+Y7T6T10+Y7T14T10+Y7T18T10+ $\mathrm{Y} 7 \mathrm{~T} 19 \mathrm{~T} 10=1$ Y7T14+Y7T6T14+Y7T10T14+Y7T18T14+ Y7T19T14=1 Y7T18+Y7T6T18+Y7T10T18+Y7T14T18+ $\mathrm{Y} 7 \mathrm{~T} 19 \mathrm{~T} 18=1$ $\mathrm{Y} 7 \mathrm{~T} 19+\mathrm{Y} 7 \mathrm{~T} 10 \mathrm{~T} 19+\mathrm{Y} 7 \mathrm{~T} 14 \mathrm{~T} 19+\mathrm{Y} 7 \mathrm{~T} 18 \mathrm{~T} 19=1$ Y8T14+Y8T15T14+Y8T18T14+Y8T22T14=1 Y8T15+Y8T14T15+Y8T18T15+Y8T22T15=1 $\mathrm{Y} 8 \mathrm{~T} 18+\mathrm{Y} 8 \mathrm{~T} 14 \mathrm{~T} 18+\mathrm{Y} 8 \mathrm{~T} 15 \mathrm{~T} 18+\mathrm{Y} 8 \mathrm{~T} 22 \mathrm{~T} 18=1$ $\mathrm{Y} 8 \mathrm{~T} 22+\mathrm{Y} 8 \mathrm{~T} 14 \mathrm{~T} 22+\mathrm{Y} 8 \mathrm{~T} 15 \mathrm{~T} 22+\mathrm{Y} 8 \mathrm{~T} 18 \mathrm{~T} 22=1$ Y9T3+Y9T11T3+Y9T14T3+Y9T18T3+ $Y 9 T 23 T 3=1$ Y9T6+Y9T11T6+Y9T14T6+Y9T15T6+ Y9T18T6=1 Y9T11+Y9T3T11+Y9T6T11+Y9T14T11+ Y9T15T11+Y9T16T11+Y9T18T11+Y9T22T11=1 Y9T14+Y9T3T14+Y9T6T14+Y9T11T14+ Y9T15T14+Y9T16T14+Y9T18T14+Y9T22T14+ Y9T23T14=1 Y9T15+Y9T6T15+Y9T11T15+Y9T14T15+ Y9T16T15+Y9T18T15+Y9T22T15=1 Y9T16+Y9T11T16+Y9T14T16+Y9T15T16+ Y9T18T16+Y9T23T16=1 Y9T18+Y9T3T18+Y9T6T18+Y9T11T18+ Y9T14T18+Y9T15T18+Y9T16T18+Y9T22T18+ Y9T23T18=1 Y9T22+Y9T11T22+Y9T14T22+Y9T15T22+ Y9T18T22+Y9T23T22=1 Y9T23+Y9T3T23+Y9T14T23+Y9T16T23+ $\mathrm{Y} 9 \mathrm{~T} 18 \mathrm{~T} 23+\mathrm{Y} 9 \mathrm{~T} 22 \mathrm{~T} 23=1$ $\mathrm{Y} 10 \mathrm{~T} 6+\mathrm{Y} 10 \mathrm{~T} 7 \mathrm{~T} 6+\mathrm{Y} 10 \mathrm{~T} 14 \mathrm{~T} 6+\mathrm{Y} 10 \mathrm{~T} 18 \mathrm{~T} 6=1$ $\mathrm{Y} 10 \mathrm{~T} 7+\mathrm{Y} 10 \mathrm{~T} 6 \mathrm{~T} 7+\mathrm{Y} 10 \mathrm{~T} 14 \mathrm{~T} 7+\mathrm{Y} 10 \mathrm{~T} 18 \mathrm{~T} 7+$ $\mathrm{Y} 10 \mathrm{~T} 19 \mathrm{~T} 7=1$ Y10T14+Y10T6T14+Y10T7T14+Y10T1814+ Y10T1914=1 Y10T18+Y10T6T18+Y10T7T18+Y10T1418+ $\mathrm{Y} 10 \mathrm{~T} 1918=1$ $\mathrm{Y} 10 \mathrm{~T} 19+\mathrm{Y} 10 \mathrm{~T} 7 \mathrm{~T} 19+\mathrm{Y} 10 \mathrm{~T} 1419+\mathrm{Y} 10 \mathrm{~T} 1819=1$ Y11T3+Y11T9T3+Y11T14T3+Y11T18T3+ $Y 11 T 21 T 3=1$ Y11T6+Y11T9T6+Y11T14T6+Y11T15T6+ $Y 11 T 18 \mathrm{~T} 6=1$ Y11T9+Y11T3T9+Y11T6T9+Y11T14T9+ $\mathrm{Y} 11 \mathrm{~T} 15 \mathrm{~T} 9+\mathrm{Y} 11 \mathrm{~T} 16 \mathrm{~T} 9+\mathrm{Y} 11 \mathrm{~T} 18 \mathrm{~T} 9+\mathrm{Y} 11 \mathrm{~T} 22 \mathrm{T9}=1$ Y11T14+Y11T3T14+Y11T6T14+Y11T9T14+ $Y 11 T 1514+Y 11 T 1614+Y 11 T 1814+Y 11 T 2214=1$ Y11T15+Y11T6T15+Y11T9T15+Y11T1415+ $Y 11 T 1615+Y 11 T 1815+Y 11 T 2215=1$ Y11T16+Y11T9T16+Y11T1416+Y11T1516+ Y11T1816=1 $\mathrm{Y} 11 \mathrm{~T} 17+\mathrm{Y} 11 \mathrm{~T} 1817+\mathrm{Y} 11 \mathrm{~T} 2117=1$ Y11T18+Y11T3T18+Y11T6T18+Y11T9T18+ Y11T1418+Y11T1518+Y11T1618+Y11T1718+ Y11T2118+Y11T2218=1 


\title{
APPENDIX B: PROBLEM FORMULATION FILE EFFICIENT COMBINED NETWORK
}

\author{
$\mathrm{Y} 11 \mathrm{~T} 21+\mathrm{Y} 11 \mathrm{~T} 3 \mathrm{~T} 21+\mathrm{Y} 11 \mathrm{~T} 1721+\mathrm{Y} 11 \mathrm{~T} 1821+$ \\ $Y 11 T 2221=1$ \\ Y11T22+Y11T9T22+Y11T1422+Y11T1522+ \\ $Y 11 T 1822+Y 11 T 2122=1$ \\ $Y 12 \mathrm{~T} 18=1$ \\ $\mathrm{Y} 13 \mathrm{~T} 14+\mathrm{Y} 13 \mathrm{~T} 1814=1$ \\ $Y 13 T 18+Y 13 T 1418=1$ \\ $\mathrm{Y} 14 \mathrm{~T} 1+\mathrm{Y} 14 \mathrm{~T} 5 \mathrm{~T} 1+\mathrm{Y} 14 \mathrm{~T} 6 \mathrm{~T} 1+\mathrm{Y} 14 \mathrm{~T} 18 \mathrm{~T} 1=1$ \\ $\mathrm{Y} 14 \mathrm{~T} 2+\mathrm{Y} 14 \mathrm{~T} 6 \mathrm{~T} 2+\mathrm{Y} 14 \mathrm{~T} 15 \mathrm{~T} 2+\mathrm{Y} 14 \mathrm{~T} 18 \mathrm{~T} 2+$ \\ $Y 14 T 19 T 2+Y 14 T 20 T 2+Y 14 T 22 T 2=1$ \\ Y14T3+Y14T9T3+Y14T11T3+Y14T18T3+ \\ $\mathrm{Y} 14 \mathrm{~T} 23 \mathrm{~T} 3=1$ \\ $\mathrm{Y} 14 \mathrm{~T} 5+\mathrm{Y} 14 \mathrm{~T} 1 \mathrm{~T} 5+\mathrm{Y} 14 \mathrm{~T} 6 \mathrm{~T} 5+\mathrm{Y} 14 \mathrm{~T} 18 \mathrm{~T} 5+$ \\ Y14T24T5=1 \\ Y14T6+Y14T1T6+Y14T2T6+Y14T5T6+ \\ Y14T7T6+Y14T9T6+Y14T10T6+Y14T11T6+ \\ $Y 14 T 15 T 6+Y 14 T 18 T 6+Y 14 T 20 T 6=1$ \\ $\mathrm{Y} 14 \mathrm{~T} 7+\mathrm{Y} 14 \mathrm{~T} 6 \mathrm{~T} 7+\mathrm{Y} 14 \mathrm{~T} 10 \mathrm{~T} 7+\mathrm{Y} 14 \mathrm{~T} 18 \mathrm{~T} 7+$ \\ $Y 14 T 19 T 7=1$ \\ $\mathrm{Y} 14 \mathrm{~T} 8+\mathrm{Y} 14 \mathrm{~T} 15 \mathrm{~T} 8+\mathrm{Y} 14 \mathrm{~T} 18 \mathrm{~T} 8+\mathrm{Y} 14 \mathrm{~T} 22 \mathrm{~T} 8=1$ \\ Y14T9+Y14T3T9+Y14T6T9+Y14T11T9+ \\ Y14T15T9+Y14T16T9+Y14T18T9+Y14T22T9+ \\ $\mathrm{Y} 14 \mathrm{~T} 23 \mathrm{~T} 9=1$ \\ Y14T10+Y14T6T10+Y14T7T10+Y14T1810+ \\ $Y 14 T 1910=1$ \\ Y14T11+Y14T3T11+Y14T6T11+Y14T9T11+ \\ $\mathrm{Y} 14 \mathrm{~T} 1511+\mathrm{Y} 14 \mathrm{~T} 1611+\mathrm{Y} 14 \mathrm{~T} 1811+\mathrm{Y} 14 \mathrm{~T} 2211=1$ \\ $\mathrm{Y} 14 \mathrm{~T} 13+\mathrm{Y} 14 \mathrm{~T} 1813=1$ \\ $\mathrm{Y} 14 \mathrm{~T} 15+\mathrm{Y} 14 \mathrm{~T} 2 \mathrm{~T} 15+\mathrm{Y} 14 \mathrm{~T} 6 \mathrm{~T} 15+\mathrm{Y} 14 \mathrm{~T} 8 \mathrm{~T} 15+$ \\ Y14T9T15+Y14T1115+Y14T1615+Y14T1815+ \\ $\mathrm{Y} 14 \mathrm{~T} 2015+\mathrm{Y} 14 \mathrm{~T} 2215=1$ \\ Y14T16+Y14T9T16+Y14T1116+Y14T1516+ \\ $\mathrm{Y} 14 \mathrm{~T} 1816+\mathrm{Y} 14 \mathrm{~T} 2316=1$ \\ Y14T18+Y14T1T18+Y14T2T18+Y14T3T18+ \\ Y14T5T18+Y14T6T18+Y14T7T18+Y14T8T18+ \\ Y14T9T18+Y14T1018+Y14T1118+Y14T1318+ \\ Y14T9T18+Y14T1018+Y14T1118+Y14T1318+ \\ $Y 14 T 2218+Y 14 T 2318+Y 14 T 2418+Y 14 T 2518=1$ \\ Y14T19+Y14T2T19+Y14T7T19+Y14T1019+ \\ $\mathrm{Y} 14 \mathrm{~T} 1819=1$ \\ $\mathrm{Y} 14 \mathrm{~T} 20+\mathrm{Y} 14 \mathrm{~T} 2 \mathrm{~T} 20+\mathrm{Y} 14 \mathrm{~T} 6 \mathrm{~T} 20+\mathrm{Y} 14 \mathrm{~T} 1520+$ \\ $\mathrm{Y} 14 \mathrm{~T} 1820+\mathrm{Y} 14 \mathrm{~T} 2220=1$ \\ $\mathrm{Y} 14 \mathrm{~T} 22+\mathrm{Y} 14 \mathrm{~T} 2 \mathrm{~T} 22+\mathrm{Y} 14 \mathrm{~T} 8 \mathrm{~T} 22+\mathrm{Y} 14 \mathrm{~T} 9 \mathrm{~T} 22+$ \\ $\mathrm{Y} 14 \mathrm{~T} 1122+\mathrm{Y} 14 \mathrm{~T} 1522+\mathrm{Y} 14 \mathrm{~T} 1822+\mathrm{Y} 14 \mathrm{~T} 2022+$ \\ $Y 14 T 2322=1$ \\ Y14T23+Y14T3T23+Y14T9T23+Y14T1623+ \\ $\mathrm{Y} 14 \mathrm{~T} 1823+\mathrm{Y} 14 \mathrm{~T} 2223=1$ \\ Y14T24+Y14T5T24+Y14T1824-1 \\ $\mathrm{Y} 14 \mathrm{~T} 25+\mathrm{Y} 14 \mathrm{~T} 1825=1$ \\ $\mathrm{Y} 15 \mathrm{~T} 2+\mathrm{Y} 15 \mathrm{~T} 6 \mathrm{~T} 2+\mathrm{Y} 15 \mathrm{~T} 14 \mathrm{~T} 2+\mathrm{Y} 15 \mathrm{~T} 18 \mathrm{~T} 2+$ \\ $Y 15 T 20 T 2+Y 15 T 22 T 2=1$ \\ Y15T6+Y15T2T6+Y15T9T6+Y15T11T6+ \\ $Y 15 T 14 T 6+Y 15 T 18 T 6+Y 15 T 20 T 6=1$ \\ $\mathrm{Y} 15 \mathrm{~T} 8+\mathrm{Y} 15 \mathrm{~T} 14 \mathrm{~T} 8+\mathrm{Y} 15 \mathrm{~T} 18 \mathrm{~T} 8+\mathrm{Y} 15 \mathrm{~T} 22 \mathrm{~T} 8=1$ \\ Y15T9+Y15T6T9+Y15T11T9+Y15T14T9+ \\ $Y 15 \mathrm{~T} 16 \mathrm{~T} 9+\mathrm{Y} 15 \mathrm{~T} 18 \mathrm{~T} 9+\mathrm{Y} 15 \mathrm{~T} 2 \mathrm{~T} 9=1$ \\ Y15T11+Y15T6T11+Y15T9T11+Y15T1411+ \\ $\mathrm{Y} 15 \mathrm{~T} 1611+\mathrm{Y} 15 \mathrm{~T} 1811+\mathrm{Y} 15 \mathrm{~T} 2211=1$ \\ Y15T14+Y15T2T14+Y15T6T14+Y15T8T14+ \\ Y15T14+Y15T2T14+Y15T6T14+Y15T8T14+ \\ Y15T2014+Y15T2214=1 \\ Y15T16+Y15T9T16+Y15T1116+Y15T1416+ \\ $Y 15 T 1816=1$ \\ $Y 15 \mathrm{~T} 18+Y 15 \mathrm{~T} 2 \mathrm{~T} 18+\mathrm{Y} 15 \mathrm{~T} 6 \mathrm{~T} 18+\mathrm{Y} 15 \mathrm{~T} 8 \mathrm{~T} 18+$ \\ Y15T9T18+Y15T1118+Y15T1418+Y15T1618+
}

$\mathrm{Y} 15 \mathrm{~T} 2018+\mathrm{Y} 15 \mathrm{~T} 2218=1$

$\mathrm{Y} 15 \mathrm{~T} 20+\mathrm{Y} 15 \mathrm{~T} 2 \mathrm{~T} 20+\mathrm{Y} 15 \mathrm{~T} 6 \mathrm{~T} 20+\mathrm{Y} 15 \mathrm{~T} 1420+$

$\mathrm{Y} 15 \mathrm{~T} 1820+\mathrm{Y} 15 \mathrm{~T} 2220=1$

Y15T22+Y15T2T22+Y15T8T22+Y15T9T22+

$Y 15 T 1122+Y 15 T 1422+Y 15 T 1822+Y 15 T 2022=1$

$\mathrm{Y} 16 \mathrm{~T} 9+\mathrm{Y} 16 \mathrm{~T} 11 \mathrm{~T} 9+\mathrm{Y} 16 \mathrm{~T} 14 \mathrm{~T} 9+\mathrm{Y} 16 \mathrm{~T} 15 \mathrm{~T} 9+$

$\mathrm{Y} 16 \mathrm{~T} 18 \mathrm{~T} 9+\mathrm{Y} 16 \mathrm{~T} 23 \mathrm{~T} 9=1$

Y16T11+Y 16T9T11+Y16T1411+Y16T1511+

$\mathrm{Y} 16 \mathrm{~T} 1811=1$

Y16T14+Y16T9T14+Y16T1114+Y16T1514+

Y16T1814+Y16T2314=1

Y16T15+Y16T9T15+Y16T1115+Y16T1415+

$\mathrm{Y} 16 \mathrm{~T} 1815=1$

$\mathrm{Y} 16 \mathrm{~T} 18+\mathrm{Y} 16 \mathrm{~T} 9 \mathrm{~T} 18+\mathrm{Y} 16 \mathrm{~T} 1118+\mathrm{Y} 16 \mathrm{~T} 1418+$

$\mathrm{Y} 16 \mathrm{~T} 1518+\mathrm{Y} 16 \mathrm{~T} 2318=1$

$\mathrm{Y} 16 \mathrm{~T} 23+\mathrm{Y} 16 \mathrm{~T} 9 \mathrm{~T} 23+\mathrm{Y} 16 \mathrm{~T} 1423+\mathrm{Y} 16 \mathrm{~T} 1823=1$

$\mathrm{Y} 17 \mathrm{~T} 4+\mathrm{Y} 17 \mathrm{~T} 18 \mathrm{~T} 4=1$

$\mathrm{Y} 17 \mathrm{~T} 11+\mathrm{Y} 17 \mathrm{~T} 1811+\mathrm{Y} 17 \mathrm{~T} 2111=1$

$\mathrm{Y} 17 \mathrm{~T} 18+\mathrm{Y} 17 \mathrm{~T} 4 \mathrm{~T} 18+\mathrm{Y} 17 \mathrm{~T} 1118+\mathrm{Y} 17 \mathrm{~T} 2118=1$

$\mathrm{Y} 17 \mathrm{~T} 21+\mathrm{Y} 17 \mathrm{~T} 1121+\mathrm{Y} 17 \mathrm{~T} 1821=1$

$\mathrm{Y} 18 \mathrm{~T} 1+\mathrm{Y} 18 \mathrm{~T} 5 \mathrm{~T} 1+\mathrm{Y} 18 \mathrm{~T} 6 \mathrm{~T} 1+\mathrm{Y} 18 \mathrm{~T} 14 \mathrm{~T} 1=1$

$\mathrm{Y} 18 \mathrm{~T} 2+\mathrm{Y} 18 \mathrm{~T} 6 \mathrm{~T} 2+\mathrm{Y} 18 \mathrm{~T} 14 \mathrm{~T} 2+\mathrm{Y} 18 \mathrm{~T} 15 \mathrm{~T} 2+$

$\mathrm{Y} 18 \mathrm{~T} 19 \mathrm{~T} 2+\mathrm{Y} 18 \mathrm{~T} 20 \mathrm{~T} 2+\mathrm{Y} 18 \mathrm{~T} 22 \mathrm{~T} 2=1$

$Y 18 T 3+Y 18 T 9 T 3+Y 18 T 11 T 3+Y 18 T 14 T 3+$

$Y 18 T 21 T 3+Y 18 T 23 T 3=1$

$\mathrm{Y} 18 \mathrm{~T} 4+\mathrm{Y} 18 \mathrm{~T} 17 \mathrm{~T} 4=1$

Y18T5+Y18T1T5+Y18T6T5+Y18T14T5+

$Y 18 T 24 T 5=1$

$\mathrm{Y} 18 \mathrm{~T} 6+\mathrm{Y} 18 \mathrm{~T} 1 \mathrm{~T} 6+\mathrm{Y} 18 \mathrm{~T} 2 \mathrm{~T} 6+\mathrm{Y} 18 \mathrm{~T} 5 \mathrm{~T} 6+$

Y18T7T6+Y18T9T6+Y18T10T6+Y18T11T6+

Y18T14T6+Y18T15T6+Y18T20T6=1

$\mathrm{Y} 18 \mathrm{~T} 7+\mathrm{Y} 18 \mathrm{~T} 6 \mathrm{~T} 7+\mathrm{Y} 18 \mathrm{~T} 10 \mathrm{~T} 7+\mathrm{Y} 18 \mathrm{~T} 14 \mathrm{~T} 7+$

$\mathrm{Y} 18 \mathrm{~T} 19 \mathrm{~T} 7=1$

$\mathrm{Y} 18 \mathrm{~T} 8+\mathrm{Y} 18 \mathrm{~T} 14 \mathrm{~T} 8+\mathrm{Y} 18 \mathrm{~T} 15 \mathrm{~T} 8+\mathrm{Y} 18 \mathrm{~T} 22 \mathrm{~T} 8=1$

Y18T9+Y18T3T9+Y18T6T9+Y18T11T9+

$\mathrm{Y} 18 \mathrm{~T} 14 \mathrm{~T} 9+\mathrm{Y} 18 \mathrm{~T} 15 \mathrm{~T} 9+\mathrm{Y} 18 \mathrm{~T} 16 \mathrm{~T} 9+\mathrm{Y} 18 \mathrm{~T} 22 \mathrm{~T} 9+$

$\mathrm{Y} 18 \mathrm{~T} 23 \mathrm{~T} 9=1$

$\mathrm{Y} 18 \mathrm{~T} 10+\mathrm{Y} 18 \mathrm{~T} 6 \mathrm{~T} 10+\mathrm{Y} 18 \mathrm{~T} 7 \mathrm{~T} 10+\mathrm{Y} 18 \mathrm{~T} 1410+$

$\mathrm{Y} 18 \mathrm{~T} 1910=1$

$\mathrm{Y} 18 \mathrm{~T} 11+\mathrm{Y} 18 \mathrm{~T} 3 \mathrm{~T} 11+\mathrm{Y} 18 \mathrm{~T} 6 \mathrm{~T} 11+\mathrm{Y} 18 \mathrm{~T} 9 \mathrm{~T} 11+$

$Y 18 T 1411+Y 18 T 1511+Y 18 T 1611+Y 18 T 1711+$

$\mathrm{Y} 18 \mathrm{~T} 2111+\mathrm{Y} 18 \mathrm{~T} 2211=1$

$\mathrm{Y} 18 \mathrm{~T} 12=1$

$\mathrm{Y} 18 \mathrm{~T} 13+\mathrm{Y} 18 \mathrm{~T} 1413=1$

Y18T14+Y18T1T14+Y18T2T14+Y18T3T14+ Y18T5T14+Y18T6T14+Y18T7T14+Y18T8T14+ Y18T9T14+Y18T1014+Y18T1114+Y18T1314+ Y18T1514+Y18T1614+Y18T1914+Y18T2014+ $\mathrm{Y} 18 \mathrm{~T} 2214+\mathrm{Y} 18 \mathrm{~T} 2314+\mathrm{Y} 18 \mathrm{~T} 2414+\mathrm{Y} 18 \mathrm{~T} 2514=1$ Y18T15+Y18T2T15+Y18T6T15+Y18T8T15+ Y18T9T15+Y18T1115+Y18T1415+Y18T1615+ $\mathrm{Y} 18 \mathrm{~T} 2015+\mathrm{Y} 18 \mathrm{~T} 2215=1$

Y18T16+Y18T9T16+Y18T1116+Y18T1416+

$\mathrm{Y} 18 \mathrm{~T} 1516+\mathrm{Y} 18 \mathrm{~T} 2316=1$

$\mathrm{Y} 18 \mathrm{~T} 17+\mathrm{Y} 18 \mathrm{~T} 4 \mathrm{~T} 17+\mathrm{Y} 18 \mathrm{~T} 1117+\mathrm{Y} 18 \mathrm{~T} 2117=1$ Y18T19+Y18T2T19+Y 18T7T19+Y18T1019+

$\mathrm{Y} 18 \mathrm{~T} 1419=1$

Y18T20+Y18T2T20+Y18T6T20+Y18T1420+

$\mathrm{Y} 18 \mathrm{~T} 1520+\mathrm{Y} 18 \mathrm{~T} 2220=1$

$\mathrm{Y} 18 \mathrm{~T} 21+\mathrm{Y} 18 \mathrm{~T} 3 \mathrm{~T} 21+\mathrm{Y} 18 \mathrm{~T} 1121+\mathrm{Y} 18 \mathrm{~T} 1721+$

$\mathrm{Y} 18 \mathrm{~T} 2221=1$

$\mathrm{Y} 18 \mathrm{~T} 22+\mathrm{Y} 18 \mathrm{~T} 2 \mathrm{~T} 22+\mathrm{Y} 18 \mathrm{~T} 8 \mathrm{~T} 22+\mathrm{Y} 18 \mathrm{~T} 9 \mathrm{~T} 22+$

Y18T1122+Y18T1422+Y18T1522+Y18T2022+

$\mathrm{Y} 18 \mathrm{~T} 2122+\mathrm{Y} 18 \mathrm{~T} 2322=1$

Y18T23+Y18T3T23+Y18T9T23+Y18T1423+ 


\section{APPENDIX B: PROBLEM FORMULATION FILE EFFICIENT COMBINED NETWORK}

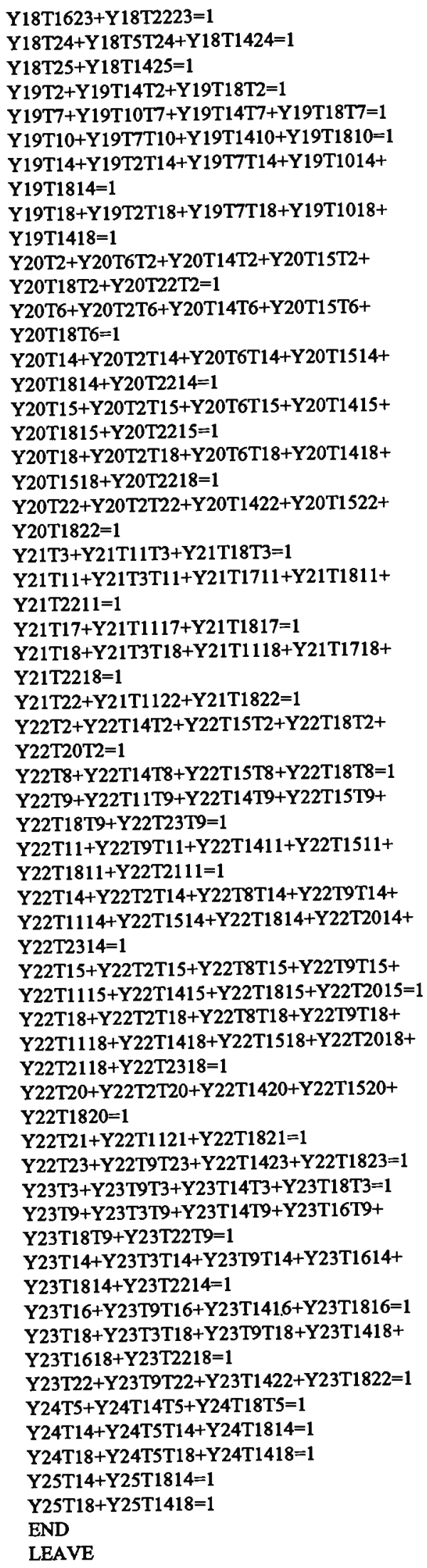


APPENDIX C 
APPENDIX C :

EFFICIENT AIRLINE NETWORK SOLUTION

SOLUTION FILE EFFICIENT AIRLINE NETWORK

\begin{tabular}{|c|c|c|}
\hline DECISION VARIABLE & VALUE & REDUCED COS? \\
\hline Y1T14T5 & 1.000000 & 0.000000 \\
\hline Y1T18T6 & 1.000000 & 0.000000 \\
\hline Y1T14 & 1.000000 & 0.000000 \\
\hline Y1T18 & 1.000000 & 0.000000 \\
\hline Y2T18T6 & 1.000000 & 0.000000 \\
\hline Y2T14 & 1.000000 & 0.000000 \\
\hline Y $2 \mathrm{~T} 14 \mathrm{~T} 15$ & 1.000000 & 0.000000 \\
\hline Y2T18 & 1.000000 & 0.000000 \\
\hline Ү 2 T 19 & 1.000000 & 0.000000 \\
\hline $\mathrm{Y} 2 \mathrm{~T} 20$ & 1.000000 & 0.000000 \\
\hline Y $2 \mathrm{~T} 14 \mathrm{~T} 22$ & 1.000000 & 0.000000 \\
\hline Y3T18T9 & 1.000000 & 0.000000 \\
\hline Y3T11 & 1.000000 & 0.000000 \\
\hline Y3T14 & 1.000000 & 0.000000 \\
\hline Y3T18 & 1.000000 & 0.000000 \\
\hline Y3Т21 & 1.000000 & 0.000000 \\
\hline Y $3 \mathrm{~T} 18 \mathrm{~T} 23$ & 1.000000 & 0.000000 \\
\hline Y4T18T17 & 1.000000 & 0.000000 \\
\hline Y $4 \mathrm{~T} 18$ & 1.000000 & 0.000000 \\
\hline Y5T14T1 & 1.000000 & 0.000000 \\
\hline Y5T18T6 & 1.000000 & 0.000000 \\
\hline Y5T14 & 1.000000 & 0.000000 \\
\hline Y5T18 & 1.000000 & 0.000000 \\
\hline Y5T18T24 & 1.000000 & 0.000000 \\
\hline Y6T18T1 & 1.000000 & 0.000000 \\
\hline Y $6 \mathrm{~T} 2$ & 1.000000 & 0.000000 \\
\hline Y6T5 & 1.000000 & 0.000000 \\
\hline Y6T7 & 1.000000 & 0.000000 \\
\hline Y6T9 & 1.000000 & 0.000000 \\
\hline Y6T10 & 1.000000 & 0.000000 \\
\hline Y6T18T11 & 1.000000 & 0.000000 \\
\hline Y $6 \mathrm{~T} 14$ & 1.000000 & 0.000000 \\
\hline Y6T15 & 1.000000 & 0.000000 \\
\hline Y6T18 & 1.000000 & 0.000000 \\
\hline Y6T18T20 & 1.000000 & 0.000000 \\
\hline Y7T18T6 & 1.000000 & 0.000000 \\
\hline Y7T19T10 & 1.000000 & 0.000000 \\
\hline Y7T14 & 1.000000 & 0.000000 \\
\hline Y7T18 & 1.000000 & 0.000000 \\
\hline Y7T19 & 1.000000 & 0.000000 \\
\hline Y8T14 & 1.000000 & 0.000000 \\
\hline Y8T22T15 & 1.000000 & 0.000000 \\
\hline Y $8 \mathrm{~T} 22 \mathrm{~T} 18$ & 1.000000 & 0.000000 \\
\hline Y8T22 & 1.000000 & 0.000000 \\
\hline Y9T18T3 & 1.000000 & 0.000000 \\
\hline Ү9T18T6 & 1.000000 & 0.000000 \\
\hline Y9T18T11 & 1.000000 & 0.000000 \\
\hline Y9T14 & 1.000000 & 0.000000 \\
\hline Y9T18T15 & 1.000000 & 0.000000 \\
\hline Y9T18T16 & 1.000000 & 0.000000 \\
\hline Y9T18 & 1.000000 & 0.000000 \\
\hline Ү9T18T22 & 1.000000 & 0.000000 \\
\hline Y9T18T23 & 1.000000 & 0.000000 \\
\hline
\end{tabular}


APPENDIX C :

EFFICIENT AIRIINE NETWORK SOLUTION

\begin{tabular}{|c|c|c|}
\hline Y10T18T6 & 1.000000 & 0.000000 \\
\hline Y10T14T7 & 1.000000 & 0.000000 \\
\hline Y10T14 & 1.000000 & 0.000000 \\
\hline Y10T18 & 1.000000 & 0.000000 \\
\hline Y10T19 & 1.000000 & 0.000000 \\
\hline Y11T3 & 1.000000 & 0.000000 \\
\hline Y11T18T6 & 1.000000 & 0.000000 \\
\hline Y11T18T9 & 1.000000 & 0.000000 \\
\hline Y11T14 & 1.000000 & 0.000000 \\
\hline Y11T1415 & 1.000000 & 0.000000 \\
\hline Y11T16 & 1.000000 & 0.000000 \\
\hline Y11T17 & 1.000000 & 0.000000 \\
\hline Y11T18 & 1.000000 & 0.000000 \\
\hline Y11T3T21 & 1.000000 & 0.000000 \\
\hline Y11T1422 & 1.000000 & 0.000000 \\
\hline Y12T18 & 1.000000 & 0.000000 \\
\hline Y13T14 & 1.000000 & 0.000000 \\
\hline Y13T18 & 1.000000 & 0.000000 \\
\hline Y14T1 & 1.000000 & 0.000000 \\
\hline Y14T2 & 1.000000 & 0.000000 \\
\hline Y14T3 & 1.000000 & 0.000000 \\
\hline Y14T5 & 1.000000 & 0.000000 \\
\hline Y14T6 & 1.000000 & 0.000000 \\
\hline $\mathrm{Y} 14 \mathrm{~T} 7$ & 1.000000 & 0.000000 \\
\hline Y14T8 & 1.000000 & 0.000000 \\
\hline $\mathrm{Y} 14 \mathrm{~T} 9$ & 1.000000 & 0.000000 \\
\hline $\mathrm{Y} 14 \mathrm{~T} 10$ & 1.000000 & 0.000000 \\
\hline Y14T11 & 1.000000 & 0.000000 \\
\hline Y14T1813 & 1.000000 & 0.000000 \\
\hline Y14T15 & 1.000000 & 0.000000 \\
\hline Y14T1116 & 1.000000 & 0.000000 \\
\hline $\mathrm{Y} 14 \mathrm{~T} 18$ & 1.000000 & 0.000000 \\
\hline Y14T7T19 & 1.000000 & 0.000000 \\
\hline $\mathrm{Y} 14 \mathrm{~T} 20$ & 1.000000 & 0.000000 \\
\hline Y14T22 & 1.000000 & 0.000000 \\
\hline $\mathrm{Y} 14 \mathrm{~T} 23$ & 1.000000 & 0.000000 \\
\hline $\mathrm{Y} 14 \mathrm{~T} 24$ & 1.000000 & 0.000000 \\
\hline Y14T25 & 1.000000 & 0.000000 \\
\hline Y15T14T2 & 1.000000 & 0.000000 \\
\hline Y15T 6 & 1.000000 & 0.000000 \\
\hline Y15T22T8 & 1.000000 & 0.000000 \\
\hline Y15T18T9 & 1.000000 & 0.000000 \\
\hline Y15T1411 & 1.000000 & 0.000000 \\
\hline Y15T14 & 1.000000 & 0.000000 \\
\hline Y15T1816 & 1.000000 & 0.000000 \\
\hline Y15T18 & 1.000000 & 0.000000 \\
\hline Y15T1420 & 1.000000 & 0.000000 \\
\hline Y15T22 & 1.000000 & 0.000000 \\
\hline Y16T18T9 & 1.000000 & 0.000000 \\
\hline Y16T1811 & 1.000000 & 0.000000 \\
\hline Y16T1814 & 1.000000 & 0.000000 \\
\hline Y16T1815 & 1.000000 & 0.000000 \\
\hline Y16T18 & 1.000000 & 0.000000 \\
\hline Y16T1823 & 1.000000 & 0.000000 \\
\hline Y17T18T4 & 1.000000 & 0.000000 \\
\hline Y17T11 & 1.000000 & 0.000000 \\
\hline Y17T18 & 1.000000 & 0.000000 \\
\hline Y17T1821 & 1.000000 & 0.000000 \\
\hline Y18T1 & 1.000000 & 0.000000 \\
\hline Ү18T2 & 1.000000 & 0.000000 \\
\hline
\end{tabular}


APPENDIX $\mathrm{C}$ :

EFFICIENT AIRLINE NETWORK SOLUTION

\begin{tabular}{|c|c|c|}
\hline Y18T3 & 1.000000 & 0.000000 \\
\hline Y18T 4 & 1.000000 & 0.000000 \\
\hline Y18T5 & 1.000000 & 0.000000 \\
\hline Y18T6 & 1.000000 & 0.000000 \\
\hline Y18T7 & 1.000000 & 0.000000 \\
\hline Y18T22T8 & 1.000000 & 0.000000 \\
\hline Y18T9 & 1.000000 & 0.000000 \\
\hline Y18T10 & 1.000000 & 0.000000 \\
\hline Y18T11 & 1.000000 & 0.000000 \\
\hline Y18T12 & 1.000000 & 0.000000 \\
\hline Y18T13 & 1.000000 & 0.000000 \\
\hline Y18T14 & 1.000000 & 0.000000 \\
\hline Y18T15 & 1.000000 & 0.000000 \\
\hline Y18T16 & 1.000000 & 0.000000 \\
\hline Y18T17 & 1.000000 & 0.000000 \\
\hline Y18T19 & 1.000000 & 0.000000 \\
\hline $\mathrm{Y} 18 \mathrm{~T} 20$ & 1.000000 & 0.000000 \\
\hline Y18T21 & 1.000000 & 0.000000 \\
\hline Y18T22 & 1.000000 & 0.000000 \\
\hline Y18T23 & 1.000000 & 0.000000 \\
\hline Y18T24 & 1.000000 & 0.000000 \\
\hline Y18T25 & 1.000000 & 0.000000 \\
\hline Y19T2 & 1.000000 & 0.000000 \\
\hline Y19T7 & 1.000000 & 0.000000 \\
\hline Y19T10 & 1.000000 & 0.000000 \\
\hline Y19T1014 & 1.000000 & 0.000000 \\
\hline Y19T18 & 1.000000 & 0.000000 \\
\hline Y20T2 & 1.000000 & 0.000000 \\
\hline Y20T18T6 & 1.000000 & 0.000000 \\
\hline Y20T2T14 & 1.000000 & 0.000000 \\
\hline Y20T15 & 1.000000 & 0.000000 \\
\hline Y20T18 & 1.000000 & 0.000000 \\
\hline Y20T1822 & 1.000000 & 0.000000 \\
\hline Y21T18T3 & 1.000000 & 0.000000 \\
\hline Y21T1811 & 1.000000 & 0.000000 \\
\hline Y21T1817 & 1.000000 & 0.000000 \\
\hline Y21T18 & 1.000000 & 0.000000 \\
\hline Y21T1822 & 1.000000 & 0.000000 \\
\hline $\mathrm{Y} 22 \mathrm{~T} 14 \mathrm{~T} 2$ & 1.000000 & 0.000000 \\
\hline Y22T 8 & 1.000000 & 0.000000 \\
\hline Y22T18T9 & 1.000000 & 0.000000 \\
\hline Y22T1811 & 1.000000 & 0.000000 \\
\hline Y22T14 & 1.000000 & 0.000000 \\
\hline Y22T15 & 1.000000 & 0.000000 \\
\hline Y22T18 & 1.000000 & 0.000000 \\
\hline Y22T 1420 & 1.000000 & 0.000000 \\
\hline Y22T1821 & 1.000000 & 0.000000 \\
\hline Y22T23 & 1.000000 & 0.000000 \\
\hline Y23T18T3 & 1.000000 & 0.000000 \\
\hline Y23T18T9 & 1.000000 & 0.000000 \\
\hline Y23T14 & 1.000000 & 0.000000 \\
\hline Y23T1816 & 1.000000 & 0.000000 \\
\hline Y23T18 & 1.000000 & 0.000000 \\
\hline Y23T22 & 1.000000 & 0.000000 \\
\hline Y24T18T5 & 1.000000 & 0.000000 \\
\hline Y24T14 & 1.000000 & 0.000000 \\
\hline $\mathrm{Y} 24 \mathrm{~T} 18$ & 1.000000 & 0.000000 \\
\hline Y25T14 & 1.000000 & 0.00000 \\
\hline Y $25 \mathrm{~T} 18$ & 1.000000 & 0.00000 \\
\hline
\end{tabular}


APPENDIX C:

EFFICIENT PASSENGERS NETWORK SOLUTION

SOLUTION FILE EFFICIENT PASSENGERS NETWORK

\begin{tabular}{|c|c|c|}
\hline DECISION VARIABLE & VALUE & REDUCED COST \\
\hline Y1T18T5 & 1.000000 & 0.000000 \\
\hline Y1T18T6 & 1.000000 & 0.000000 \\
\hline Y1T18T14 & 1.000000 & 0.000000 \\
\hline Y1T18 & 1.000000 & 0.000000 \\
\hline Y2T18T6 & 1.000000 & 0.000000 \\
\hline Y2T 14 & 1.000000 & 0.000000 \\
\hline Y2T14T15 & 1.000000 & 0.000000 \\
\hline Y2T18 & 1.000000 & 0.000000 \\
\hline Y2T14T19 & 1.000000 & 0.000000 \\
\hline Y2T18T20 & 1.000000 & 0.000000 \\
\hline Y $2 \mathrm{~T} 14 \mathrm{~T} 22$ & 1.000000 & 0.000000 \\
\hline Y3T18T9 & 1.000000 & 0.000000 \\
\hline Ү3Т18T11 & 1.000000 & 0.000000 \\
\hline Ү3Т18T14 & 1.000000 & 0.000000 \\
\hline Y3T18 & 1.000000 & 0.000000 \\
\hline Ү3Т21 & 1.000000 & 0.000000 \\
\hline Y3T18T23 & 1.000000 & 0.000000 \\
\hline Y4T18T17 & 1.000000 & 0.000000 \\
\hline Y4T18 & 1.000000 & 0.000000 \\
\hline Y5T18T1 & 1.000000 & 0.000000 \\
\hline Y5T18T6 & 1.000000 & 0.000000 \\
\hline Y5T18T14 & 1.000000 & 0.000000 \\
\hline Y 5T18 & 1.000000 & 0.000000 \\
\hline Y5T18T24 & 1.000000 & 0.000000 \\
\hline Y6T18T1 & 1.000000 & 0.000000 \\
\hline Y6T18T2 & 1.000000 & 0.000000 \\
\hline Y6T18T5 & 1.000000 & 0.000000 \\
\hline Y6T18T7 & 1.000000 & 0.000000 \\
\hline Y6T18T9 & 1.000000 & 0.000000 \\
\hline Y6T18T10 & 1.000000 & 0.000000 \\
\hline Y6T18T11 & 1.000000 & 0.000000 \\
\hline Y6T14 & 1.000000 & 0.000000 \\
\hline Y6T14T15 & 1.000000 & 0.000000 \\
\hline Y6T18 & 1.000000 & 0.000000 \\
\hline Y6T18T20 & 1.000000 & 0.000000 \\
\hline Y7T18T6 & 1.000000 & 0.000000 \\
\hline Y7T18T10 & 1.000000 & 0.000000 \\
\hline Y7T14 & 1.000000 & 0.000000 \\
\hline Y7T18 & 1.000000 & 0.000000 \\
\hline Y7T14T19 & 1.000000 & 0.000000 \\
\hline Y8T14 & 1.000000 & 0.000000 \\
\hline Y8T14T15 & 1.000000 & 0.000000 \\
\hline Y8T14T18 & 1.000000 & 0.000000 \\
\hline Y $8 \mathrm{~T} 14 \mathrm{~T} 22$ & 1.000000 & 0.000000 \\
\hline Y9T18T3 & 1.000000 & 0.000000 \\
\hline Ү9т18T6 & 1.000000 & 0.000000 \\
\hline Y9T18T11 & 1.000000 & 0.000000 \\
\hline Y9T18T14 & 1.000000 & 0.000000 \\
\hline Y9T18T15 & 1.000000 & 0.000000 \\
\hline Y9T18T16 & 1.000000 & 0.000000 \\
\hline Y9T18 & 1.000000 & 0.000000 \\
\hline Ү9Т18Т22 & 1.000000 & 0.000000 \\
\hline Ү9T18T23 & 1.000000 & 0.000000 \\
\hline
\end{tabular}


APPENDIX C:

EFEICIENT PASSENGERS NETWORK SOLUTION

\begin{tabular}{|c|c|c|}
\hline Y10T18T6 & 1.000000 & 0.000000 \\
\hline Y10T18T7 & 1.000000 & 0.000000 \\
\hline Y10T14 & 1.000000 & 0.000000 \\
\hline Y10T18 & 1.000000 & 0.000000 \\
\hline Y10T1419 & 1.000000 & 0.000000 \\
\hline $\mathrm{Y} 11 \mathrm{~T} 18 \mathrm{~T} 3$ & 1.000000 & 0.000000 \\
\hline Y11T18T6 & 1.000000 & 0.000000 \\
\hline Y11T18T9 & 1.000000 & 0.000000 \\
\hline Y11T1814 & 1.000000 & 0.000000 \\
\hline Y11T1815 & 1.000000 & 0.000000 \\
\hline Y11T1816 & 1.000000 & 0.000000 \\
\hline Y11T1817 & 1.000000 & 0.000000 \\
\hline Y11T18 & 1.000000 & 0.000000 \\
\hline Y11T21 & 1.000000 & 0.000000 \\
\hline Y11T1822 & 1.000000 & 0.000000 \\
\hline Y12T18 & 1.000000 & 0.000000 \\
\hline Y13T1814 & 1.000000 & 0.000000 \\
\hline Y13T18 & 1.000000 & 0.000000 \\
\hline $\mathrm{Y} 14 \mathrm{~T} 18 \mathrm{~T} 1$ & 1.000000 & 0.000000 \\
\hline Y14T2 & 1.000000 & 0.000000 \\
\hline $\mathrm{Y} 14 \mathrm{~T} 18 \mathrm{~T} 3$ & 1.000000 & 0.000000 \\
\hline Y14T18T5 & 1.000000 & 0.000000 \\
\hline $\mathrm{Y} 14 \mathrm{~T} 6$ & 1.000000 & 0.000000 \\
\hline Y14T7 & 1.000000 & 0.000000 \\
\hline Y14T8 & 1.000000 & 0.000000 \\
\hline Y14T18T9 & 1.000000 & 0.000000 \\
\hline Y14T10 & 1.000000 & 0.000000 \\
\hline Y14T1811 & 1.000000 & 0.000000 \\
\hline Y14T1813 & 1.000000 & 0.000000 \\
\hline Y14T15 & 1.000000 & 0.000000 \\
\hline Y14T1816 & 1.000000 & 0.000000 \\
\hline $\mathrm{Y} 14 \mathrm{~T} 18$ & 1.000000 & 0.000000 \\
\hline Y14T19 & 1.000000 & 0.000000 \\
\hline Y14T1820 & 1.000000 & 0.000000 \\
\hline Y14T22 & 1.000000 & 0.000000 \\
\hline Y14T1823 & 1.000000 & 0.000000 \\
\hline Y14T1824 & 1.000000 & 0.000000 \\
\hline Y14T25 & 1.000000 & 0.000000 \\
\hline $\mathrm{Y} 15 \mathrm{~T} 14 \mathrm{~T} 2$ & 1.000000 & 0.000000 \\
\hline Y15T14T6 & 1.000000 & 0.000000 \\
\hline $\mathrm{Y} 15 \mathrm{~T} 14 \mathrm{~T} 8$ & 1.000000 & 0.000000 \\
\hline Y15T18T9 & 1.000000 & 0.000000 \\
\hline Y15T1811 & 1.000000 & 0.000000 \\
\hline Y15T14 & 1.000000 & 0.000000 \\
\hline Y15T1816 & 1.000000 & 0.000000 \\
\hline Y15T18 & 1.000000 & 0.000000 \\
\hline Y15T1820 & 1.000000 & 0.000000 \\
\hline Y15T1422 & 1.000000 & 0.000000 \\
\hline Y16T18T9 & 1.000000 & 0.000000 \\
\hline Y16T1811 & 1.000000 & 0.000000 \\
\hline Y16T1814 & 1.000000 & 0.000000 \\
\hline Y16T1815 & 1.000000 & 0.000000 \\
\hline Y16T18 & 1.000000 & 0.000000 \\
\hline Y16T1823 & 1.000000 & 0.000000 \\
\hline Y17T18T4 & 1.000000 & 0.000000 \\
\hline Y17T1811 & 1.000000 & 0.000000 \\
\hline $\mathrm{Y} 17 \mathrm{~T} 18$ & 1.000000 & 0.000000 \\
\hline $\mathrm{Y} 17 \mathrm{~T} 21$ & 1.000000 & 0.000000 \\
\hline Y18T1 & 1.000000 & 0.000000 \\
\hline Y18T2 & 1.000000 & 0.000000 \\
\hline
\end{tabular}


APPENDIX C:

EFFICIENT PASSENGERS NETWORK SOLUTION

\begin{tabular}{|c|c|c|}
\hline Y18T3 & 1.000000 & 0.000000 \\
\hline Y18T4 & 1.000000 & 0.000000 \\
\hline Y18T5 & 1.000000 & 0.000000 \\
\hline Y18T6 & 1.000000 & 0.000000 \\
\hline Y18T7 & 1.000000 & 0.000000 \\
\hline Y18T14T8 & 1.000000 & 0.000000 \\
\hline Y18T9 & 1.000000 & 0.000000 \\
\hline Y18T10 & 1.000000 & 0.000000 \\
\hline Y18TII & 1.000000 & 0.000000 \\
\hline Y18T12 & 1.000000 & 0.000000 \\
\hline Y18T13 & 1.000000 & 0.000000 \\
\hline Y18T14 & 1.000000 & 0.000000 \\
\hline Y18T15 & 1.000000 & 0.000000 \\
\hline Y18T16 & 1.000000 & 0.000000 \\
\hline Y18T17 & 1.000000 & 0.000000 \\
\hline Y18T1419 & 1.000000 & 0.000000 \\
\hline Y18T20 & 1.000000 & 0.000000 \\
\hline Y18T3T21 & 1.000000 & 0.000000 \\
\hline Y18T22 & 1.000000 & 0.000000 \\
\hline Y18T23 & 1.000000 & 0.000000 \\
\hline Y18T24 & 1.000000 & 0.000000 \\
\hline Y18T1425 & 1.000000 & 0.000000 \\
\hline Y19T14T2 & 1.000000 & 0.000000 \\
\hline Y19T14T7 & 1.000000 & 0.000000 \\
\hline Y19T1410 & 1.000000 & 0.000000 \\
\hline Y19T14 & 1.000000 & 0.000000 \\
\hline Y19T1418 & 1.000000 & 0.000000 \\
\hline Y20T18T2 & 1.000000 & 0.000000 \\
\hline Y20T18T6 & 1.000000 & 0.000000 \\
\hline Y20T1814 & 1.000000 & 0.000000 \\
\hline Y20T1815 & 1.000000 & 0.000000 \\
\hline Y20T18 & 1.000000 & 0.000000 \\
\hline Y20T1822 & 1.000000 & 0.000000 \\
\hline Y21T3 & 1.000000 & 0.000000 \\
\hline Y21T11 & 1.000000 & 0.000000 \\
\hline Y21T17 & 1.000000 & 0.000000 \\
\hline Y21T18 & 1.000000 & 0.000000 \\
\hline Y $21 \mathrm{~T} 22$ & 1.000000 & 0.000000 \\
\hline $\mathrm{Y} 22 \mathrm{~T} 14 \mathrm{~T} 2$ & 1.000000 & 0.000000 \\
\hline Y22T14T8 & 1.000000 & 0.000000 \\
\hline Ү22Т18T9 & 1.000000 & 0.000000 \\
\hline Y22T1811 & 1.000000 & 0.000000 \\
\hline $\mathrm{Y} 22 \mathrm{~T} 14$ & 1.000000 & 0.000000 \\
\hline Y22T1415 & 1.000000 & 0.000000 \\
\hline Y22T18 & 1.000000 & 0.000000 \\
\hline Y22T1820 & 1.000000 & 0.000000 \\
\hline Y $22 \mathrm{~T} 21$ & 1.000000 & 0.000000 \\
\hline Y22T1823 & 1.000000 & 0.000000 \\
\hline Y23T18T3 & 1.000000 & 0.000000 \\
\hline Y23Т18T9 & 1.000000 & 0.000000 \\
\hline Y23T1814 & 1.000000 & 0.000000 \\
\hline Y23T1816 & 1.000000 & 0.000000 \\
\hline Y23T18 & 1.000000 & 0.000000 \\
\hline Y23T1822 & 1.000000 & 0.000000 \\
\hline Y24T18T5 & 1.000000 & 0.000000 \\
\hline Y24T1814 & 1.000000 & 0.000000 \\
\hline Y24T18 & 1.000000 & 0.000000 \\
\hline Y25T 14 & 1.000000 & 0.000000 \\
\hline Y25T1418 & 1.000000 & 0.000000 \\
\hline
\end{tabular}


APPENDIX C:

EFFICIENT COMBINED NETWORK SOLUTION

SOLUTION FILE EFFICIENT COMBINED NETWORK

\begin{tabular}{|c|c|c|}
\hline DECISION VARIABLE & VALUE & REDUCED COST \\
\hline Y1T18T5 & 1.000000 & 0.000000 \\
\hline Y1T18T6 & 1.000000 & 0.000000 \\
\hline Y1T14 & 1.000000 & 0.000000 \\
\hline Y1T18 & 1.000000 & 0.000000 \\
\hline Ү2Т18T6 & 1.000000 & 0.000000 \\
\hline Y2T14 & 1.000000 & 0.000000 \\
\hline Y $2 \mathrm{~T} 14 \mathrm{~T} 15$ & 1.000000 & 0.000000 \\
\hline Y2T18 & 1.000000 & 0.000000 \\
\hline Ү2Т19 & 1.000000 & 0.000000 \\
\hline Y2T 20 & 1.000000 & 0.000000 \\
\hline Y $2 \mathrm{~T} 14 \mathrm{~T} 22$ & 1.000000 & 0.000000 \\
\hline Ү3Т18T9 & 1.000000 & 0.000000 \\
\hline Y3T18T11 & 1.000000 & 0.000000 \\
\hline Y3T14 & 1.000000 & 0.000000 \\
\hline Ү3Т18 & 1.000000 & 0.000000 \\
\hline Ү3Т21 & 1.000000 & 0.000000 \\
\hline Y 3 T 18T23 & 1.000000 & 0.000000 \\
\hline Y4T18T17 & 1.000000 & 0.000000 \\
\hline Y4T18 & 1.000000 & 0.000000 \\
\hline Y5T14T1 & 1.000000 & 0.000000 \\
\hline Y5T18T6 & 1.000000 & 0.000000 \\
\hline Y5T14 & 1.000000 & 0.000000 \\
\hline Y5T18 & 1.000000 & 0.000000 \\
\hline Y5T18T24 & 1.000000 & 0.000000 \\
\hline Y6T18T1 & 1.000000 & 0.000000 \\
\hline Y6T18T2 & 1.000000 & 0.000000 \\
\hline Y6T18T5 & 1.000000 & 0.000000 \\
\hline Y6T18T7 & 1.000000 & 0.000000 \\
\hline Y6T18T9 & 1.000000 & 0.000000 \\
\hline Y6T18T10 & 1.000000 & 0.000000 \\
\hline Y6T18T11 & 1.000000 & 0.000000 \\
\hline Y6T14 & 1.000000 & 0.000000 \\
\hline Y6T18T15 & 1.000000 & 0.000000 \\
\hline Y6T18 & 1.000000 & 0.000000 \\
\hline Y6T18T20 & 1.000000 & 0.000000 \\
\hline Y7T18T6 & 1.000000 & 0.000000 \\
\hline Y7TI8T10 & 1.000000 & 0.000000 \\
\hline Y7T14 & 1.000000 & 0.000000 \\
\hline Y7T18 & 1.000000 & 0.000000 \\
\hline Y7T18T19 & 1.000000 & 0.000000 \\
\hline Y8T14 & 1.000000 & 0.000000 \\
\hline Y8T14T15 & 1.000000 & 0.000000 \\
\hline Y8T14T18 & 1.000000 & 0.000000 \\
\hline Y $8 \mathrm{~T} 14 \mathrm{~T} 22$ & 1.000000 & 0.000000 \\
\hline Ү9T18T3 & 1.000000 & 0.000000 \\
\hline Ү9Т18T6 & 1.000000 & 0.000000 \\
\hline Y9T18T11 & 1.000000 & 0.000000 \\
\hline Y9T14 & 1.000000 & 0.000000 \\
\hline Y9T18T15 & 1.000000 & 0.000000 \\
\hline Ү9Т18T16 & 1.000000 & 0.000000 \\
\hline Y9T18 & 1.000000 & 0.000000 \\
\hline Ү9T18T22 & 1.000000 & 0.000000 \\
\hline Y9T18T23 & 1.000000 & 0.000000 \\
\hline
\end{tabular}


APPENDIX C:

EFFICIENT COMBINED NETWORK SOLUTION

\begin{tabular}{|c|c|c|}
\hline Y10T18T6 & 1.000000 & 0.000000 \\
\hline Y10T14T7 & 1.000000 & 0.000000 \\
\hline Y10T 14 & 1.000000 & 0.000000 \\
\hline Y10T18 & 1.000000 & 0.000000 \\
\hline Y10T1819 & 1.000000 & 0.000000 \\
\hline Y11T18T3 & 1.000000 & 0.000000 \\
\hline Y11T18T6 & 1.000000 & 0.000000 \\
\hline Y11T18T9 & 1.000000 & 0.000000 \\
\hline Y11T14 & 1.000000 & 0.000000 \\
\hline Y11T1815 & 1.000000 & 0.000000 \\
\hline Y11T1816 & 1.000000 & 0.000000 \\
\hline Y11T1817 & 1.000000 & 0.000000 \\
\hline Y11T18 & 1.000000 & 0.000000 \\
\hline Y11T1821 & 1.000000 & 0.000000 \\
\hline Y11T1822 & 1.000000 & 0.000000 \\
\hline Y12T18 & 1.000000 & 0.000000 \\
\hline Y13T1814 & 1.000000 & 0.000000 \\
\hline Y13T18 & 1.000000 & 0.000000 \\
\hline Y14T1 & 1.000000 & 0.000000 \\
\hline $\mathrm{Y} 14 \mathrm{~T} 2$ & 1.000000 & 0.000000 \\
\hline Y14T3 & 1.000000 & 0.000000 \\
\hline Y14T5 & 1.000000 & 0.000000 \\
\hline Y14T6 & 1.000000 & 0.000000 \\
\hline Y14T7 & 1.000000 & 0.000000 \\
\hline Y14T8 & 1.000000 & 0.000000 \\
\hline Y14T9 & 1.000000 & 0.000000 \\
\hline Y14T10 & 1.000000 & 0.000000 \\
\hline Y14T11 & 1.000000 & 0.000000 \\
\hline Y14T1813 & 1.000000 & 0.000000 \\
\hline Y14T15 & 1.000000 & 0.000000 \\
\hline Y14T1816 & 1.000000 & 0.000000 \\
\hline Y14T18 & 1.000000 & 0.000000 \\
\hline Y14T2T19 & 1.000000 & 0.000000 \\
\hline Y14T1820 & 1.000000 & 0.000000 \\
\hline $\mathrm{Y} 14 \mathrm{~T} 22$ & 1.000000 & 0.000000 \\
\hline Y14T1823 & 1.000000 & 0.000000 \\
\hline Y14T1824 & 1.000000 & 0.000000 \\
\hline Y14T25 & 1.000000 & 0.000000 \\
\hline Y15T14T2 & 1.000000 & 0.000000 \\
\hline Y15T18T6 & 1.000000 & 0.000000 \\
\hline Y15T14T8 & 1.000000 & 0.000000 \\
\hline Y15T18T9 & 1.000000 & 0.000000 \\
\hline Y15T1811 & 1.000000 & 0.000000 \\
\hline Y15T14 & 1.000000 & 0.000000 \\
\hline Y15T1816 & 1.000000 & 0.000000 \\
\hline Y15T18 & 1.000000 & 0.000000 \\
\hline Y15T1820 & 1.000000 & 0.000000 \\
\hline Y15T1422 & 1.000000 & 0.000000 \\
\hline Y16T18T9 & 1.000000 & 0.000000 \\
\hline Y16T1811 & 1.000000 & 0.000000 \\
\hline Y16T1814 & 1.000000 & 0.000000 \\
\hline Y16T1815 & 1.000000 & 0.000000 \\
\hline Y16T18 & 1.000000 & 0.000000 \\
\hline Y16T1823 & 1.000000 & 0.000000 \\
\hline Y17T18T4 & 1.000000 & 0.000000 \\
\hline Y17T1811 & 1.000000 & 0.000000 \\
\hline Y17T18 & 1.000000 & 0.000000 \\
\hline Y17T1821 & 1.000000 & 0.000000 \\
\hline Y18T1 & 1.000000 & 0.000000 \\
\hline Y18T2 & 1.000000 & 0.000000 \\
\hline
\end{tabular}


APPENDIX C:

EFFICIENT COMBINED NETWORK SOLUTION

\begin{tabular}{|c|c|c|}
\hline Y18T3 & 1.000000 & 0.000000 \\
\hline Y18T4 & 1.000000 & 0.000000 \\
\hline Y18T5 & 1.000000 & 0.000000 \\
\hline Y18T6 & 1.000000 & 0.000000 \\
\hline Y18T7 & 1.000000 & 0.000000 \\
\hline Y18T14T8 & 1.000000 & 0.000000 \\
\hline Y18T9 & 1.000000 & 0.000000 \\
\hline Y18T10 & 1.000000 & 0.000000 \\
\hline Y18T11 & 1.000000 & 0.000000 \\
\hline Y18T12 & 1.000000 & 0.000000 \\
\hline Y18T13 & 1.000000 & 0.000000 \\
\hline Y18T14 & 1.000000 & 0.000000 \\
\hline Y18T15 & 1.000000 & 0.000000 \\
\hline Y18T16 & 1.000000 & 0.000000 \\
\hline Y18T17 & 1.000000 & 0.000000 \\
\hline Y18T19 & 1.000000 & 0.000000 \\
\hline Y18T20 & 1.000000 & 0.000000 \\
\hline Y18T21 & 1.000000 & 0.000000 \\
\hline Y18T22 & 1.000000 & 0.000000 \\
\hline Y18T23 & 1.000000 & 0.000000 \\
\hline Y18T24 & 1.000000 & 0.000000 \\
\hline Y18T1425 & 1.000000 & 0.000000 \\
\hline Y19T2 & 1.000000 & 0.000000 \\
\hline Y19T14T7 & 1.000000 & 0.000000 \\
\hline Y19T10 & 1.000000 & 0.000000 \\
\hline Y19T14 & 1.000000 & 0.000000 \\
\hline Y19T1018 & 1.000000 & 0.000000 \\
\hline Y20T2 & 1.000000 & 0.000000 \\
\hline Y20T18T6 & 1.000000 & 0.000000 \\
\hline Y20T1814 & 1.000000 & 0.000000 \\
\hline Y20T1815 & 1.000000 & 0.000000 \\
\hline Y20T18 & 1.000000 & 0.000000 \\
\hline Y20T1822 & 1.000000 & 0.000000 \\
\hline Y21T18T3 & 1.000000 & 0.000000 \\
\hline Y21T1811 & 1.000000 & 0.000000 \\
\hline Y21T1817 & 1.000000 & 0.000000 \\
\hline Y21T18 & 1.000000 & 0.000000 \\
\hline Y $21 \mathrm{~T} 1822$ & 1.000000 & 0.000000 \\
\hline Y22T14T2 & 1.000000 & 0.000000 \\
\hline$Y 22 \mathrm{~T} 14 \mathrm{~T} 8$ & 1.000000 & 0.000000 \\
\hline Y22T18T9 & 1.000000 & 0.000000 \\
\hline Y22T1811 & 1.000000 & 0.000000 \\
\hline Y22T14 & 1.000000 & 0.000000 \\
\hline $\mathrm{Y} 22 \mathrm{~T} 1415$ & 1.000000 & 0.000000 \\
\hline Y22T18 & 1.000000 & 0.000000 \\
\hline Y22T1820 & 1.000000 & 0.000000 \\
\hline Y22T1821 & 1.000000 & 0.000000 \\
\hline Y22T1823 & 1.000000 & 0.000000 \\
\hline Y23T18T3 & 1.000000 & 0.000000 \\
\hline Y23T18T9 & 1.000000 & 0.000000 \\
\hline Y23T 1814 & 1.000000 & 0.000000 \\
\hline Y23T1816 & 1.000000 & 0.000000 \\
\hline Y23T18 & 1.000000 & 0.000000 \\
\hline Y23T1822 & 1.000000 & 0.000000 \\
\hline Y24T18T5 & 1.000000 & 0.000000 \\
\hline Y24T1814 & 1.000000 & 0.000000 \\
\hline Y24T18 & 1.000000 & 0.000000 \\
\hline Y25T14 & 1.000000 & 0.000000 \\
\hline Y25T1418 & 1.000000 & 0.00000 \\
\hline
\end{tabular}


APPENDIX D 


\section{ANALYSIS FILE EXISTING NETWORK}

\begin{tabular}{|c|c|c|c|c|c|c|c|c|}
\hline \multicolumn{2}{|c|}{ Link } & \multirow{2}{*}{$\begin{array}{l}\text { Total } \\
\text { Flow }\end{array}$} & \multirow{2}{*}{$\begin{array}{l}\text { Total } \\
\text { Flights }\end{array}$} & \multirow{2}{*}{$\begin{array}{l}\text { Schedule } \\
\text { Delay }\end{array}$} & \multirow{2}{*}{$\begin{array}{l}\text { Total } \\
\text { Cost }\end{array}$} & \multirow{2}{*}{$\begin{array}{l}\text { Cost per } \\
\text { Seat Mile }\end{array}$} & \multirow{2}{*}{$\begin{array}{l}\text { Cost per } \\
\text { Pax Mile }\end{array}$} & \multirow{2}{*}{$\begin{array}{l}\text { Load } \\
\text { Factor }\end{array}$} \\
\hline Fr & To & & & & & & & \\
\hline 1 & 5 & 2 & 1 & 49.00 & 8330.00 & 0.65 & 33.32 & 0.02 \\
\hline 1 & 14 & 404 & 2 & 24.50 & 74260.00 & 1.36 & 1.73 & 0.78 \\
\hline 1 & 18 & 808 & 5 & 9.80 & 216150.00 & 0.59 & 0.74 & 0.80 \\
\hline 2 & 14 & 3134 & 16 & 3.06 & 730720.00 & 0.79 & 0.82 & 0.97 \\
\hline 2 & 18 & 3596 & 18 & 2.72 & 1057320.00 & 0.63 & 0.64 & 0.99 \\
\hline 2 & 19 & 77 & 1 & 49.00 & 15680.00 & 0.52 & 0.68 & 0.75 \\
\hline 2 & 20 & 212 & 2 & 24.50 & 60600.00 & 0.66 & 1.25 & 0.52 \\
\hline 3 & 9 & 19 & 1 & 49.00 & 24000.00 & 0.71 & 3.79 & 0.19 \\
\hline 3 & 11 & 58 & 1 & 49.00 & 18750.00 & 1.00 & 1.76 & 0.57 \\
\hline 3 & 14 & 173 & 2 & 24.50 & 57120.00 & 0.51 & 0.60 & 0.85 \\
\hline 3 & 18 & 577 & 6 & 8.17 & 195960.00 & 0.66 & 0.70 & 0.94 \\
\hline 3 & 21 & 28 & 1 & 49.00 & 9380.00 & 0.62 & 2.25 & 0.27 \\
\hline 3 & 23 & 19 & 1 & 49.00 & 18040.00 & 1.12 & 6.01 & 0.19 \\
\hline 4 & 17 & 4 & 1 & 49.00 & 20160.00 & 0.31 & 7.88 & 0.04 \\
\hline 4 & 18 & 385 & 4 & 12.25 & 88880.00 & 0.99 & 1.05 & 0.94 \\
\hline 5 & 1 & 2 & 1 & 49.00 & 8330.00 & 0.65 & 33.32 & 0.02 \\
\hline 5 & 6 & 58 & 1 & 49.00 & 32640.00 & 0.48 & 0.85 & 0.57 \\
\hline 5 & 14 & 443 & 5 & 9.80 & 87900.00 & 0.76 & 0.87 & 0.87 \\
\hline 5 & 18 & 615 & 7 & 7.00 & 143080.00 & 0.56 & 0.64 & 0.86 \\
\hline 5 & 24 & 65 & 1 & 49.00 & 10980.00 & 0.72 & 1.13 & 0.64 \\
\hline 6 & 5 & 77 & 1 & 49.00 & 28800.00 & 0.43 & 0.57 & 0.75 \\
\hline 6 & 9 & 173 & 2 & 24.50 & 43500.00 & 0.55 & 0.64 & 0.85 \\
\hline 6 & 10 & 192 & 2 & 24.50 & 65240.00 & 0.40 & 0.42 & 0.94 \\
\hline 6 & 14 & 4231 & 17 & 2.88 & 1645600.00 & 0.50 & 0.51 & 0.98 \\
\hline 6 & 15 & 346 & 2 & 24.50 & 111840.00 & 0.50 & 0.52 & 0.96 \\
\hline 6 & 18 & 8499 & 34 & 1.44 & 1317840.00 & 0.60 & 0.60 & 1.00 \\
\hline 7 & 10 & 19 & 1 & 49.00 & 12750.00 & 0.79 & 4.22 & 0.19 \\
\hline 7 & 14 & 712 & 7 & 7.00 & 168840.00 & 0.61 & 0.61 & 1.00 \\
\hline 7 & 18 & 808 & 8 & 6.12 & 191760.00 & 0.46 & 0.46 & 0.99 \\
\hline 7 & 19 & 173 & 2 & 24.50 & 24480.00 & 0.76 & 0.90 & 0.85 \\
\hline 8 & 14 & 134 & 2 & 24.50 & 49000.00 & 0.74 & 1.13 & 0.66 \\
\hline 8 & 15 & 19 & 1 & 49.00 & 21160.00 & 0.88 & 4.74 & 0.19 \\
\hline 8 & 22 & 38 & 1 & 49.00 & 16030.00 & 0.90 & 2.41 & 0.37 \\
\hline 9 & 3 & 19 & 1 & 49.00 & 20130.00 & 0.59 & 3.18 & 0.19 \\
\hline 9 & 6 & 173 & 2 & 24.50 & 60900.00 & 0.76 & 0.90 & 0.85 \\
\hline 9 & 11 & 4 & 1 & 49.00 & 12780.00 & 0.72 & 18.26 & 0.04 \\
\hline 9 & 14 & 1057 & 6 & 8.17 & 306720.00 & 0.57 & 0.66 & 0.88 \\
\hline 9 & 15 & 77 & 1 & 49.00 & 49950.00 & 0.70 & 2.28 & 0.31 \\
\hline 9 & 16 & 134 & 2 & 24.50 & 39600.00 & 0.53 & 0.81 & 0.66 \\
\hline 9 & 18 & 1115 & 6 & 8.17 & 129540.00 & 0.51 & 0.55 & 0.92 \\
\hline 9 & 22 & 38 & 1 & 49.00 & 22880.00 & 0.48 & 1.29 & 0.37 \\
\hline 9 & 23 & 23 & 1 & 49.00 & 22350.00 & 0.41 & 1.84 & 0.23 \\
\hline 10 & 6 & 212 & 3 & 16.33 & 90210.00 & 0.36 & 0.53 & 0.69 \\
\hline 10 & 7 & 19 & 1 & 49.00 & 12750.00 & 0.79 & 4.22 & 0.19 \\
\hline 10 & 14 & 1712 & 17 & 2.88 & 387600.00 & 0.67 & 0.67 & 0.99 \\
\hline 10 & 18 & 1846 & 19 & 2.58 & 478989.97 & 0.44 & 0.46 & 0.95 \\
\hline 10 & 19 & 192 & 2 & 24.50 & 34560.00 & 1.13 & 1.20 & 0.94 \\
\hline 11 & 3 & 58 & 1 & 49.00 & 15120.00 & 0.81 & 1.42 & 0.57 \\
\hline 11 & 9 & 6 & 1 & 49.00 & 12750.00 & 0.71 & 12.14 & 0.06 \\
\hline 11 & 14 & 365 & 3 & 16.33 & 104850.00 & 0.49 & 0.73 & 0.68 \\
\hline
\end{tabular}


APPENDIX D:

EXISTING NETWORK ANALYSIS

\begin{tabular}{|c|c|c|c|c|c|c|c|c|}
\hline 11 & 15 & 19 & 1 & 49.00 & 29820.00 & 0.72 & 6.79 & 0.11 \\
\hline 11 & 16 & 38 & 1 & 49.00 & 16820.00 & 0.67 & 1.81 & 0.37 \\
\hline 11 & 17 & 38 & 1 & 49.00 & 12920.00 & 0.66 & 1.77 & 0.37 \\
\hline 11 & 18 & 1480 & 9 & 5.44 & 302850.00 & 0.48 & 0.53 & 0.5 \\
\hline 11 & 21 & 5 & 1 & 49.00 & 16950.00 & 0.57 & 11.65 & 0. \\
\hline 11 & 22 & 19 & 1 & 49.00 & 49450.00 & 0.63 & 8.51 & 0.0 \\
\hline 12 & 18 & 154 & 2 & 24.50 & 27840.00 & 0.56 & 0.74 & 0.7 \\
\hline 13 & 18 & 346 & 4 & 12.25 & 63720.00 & 0.82 & 0.96 & 0. \\
\hline 14 & 1 & 442 & 2 & 24.50 & 74480.00 & 1.36 & 1.59 & $0 . \varepsilon$ \\
\hline 14 & 2 & 3154 & 16 & 3.06 & 655360.00 & 0.71 & 0.73 & 0.5 \\
\hline 14 & 3 & 173 & 2 & 24.50 & 55080.00 & 0.49 & 0.58 & $0.8-2$ \\
\hline 14 & 5 & 462 & 5 & 9.80 & 90150.00 & 0.78 & 0.86 & 0.5 \\
\hline 14 & 6 & 4327 & 18 & 2.72 & 1558080.00 & 0.45 & 0.47 & 0.5 \\
\hline 14 & 7 & 731 & 8 & 6.12 & 172480.00 & 0.54 & 0.61 & 0.5 \\
\hline 14 & 8 & 115 & 2 & 24.50 & 49280.00 & 0.75 & 1.32 & 0.0 \\
\hline 14 & 9 & 1077 & 6 & 8.17 & 252960.00 & 0.47 & 0.53 & $0 . \varepsilon$ \\
\hline 14 & 10 & 1673 & 17 & 2.88 & 345440.00 & 0.59 & 0.61 & 0. \\
\hline 14 & 11 & 365 & 3 & 16.33 & 81720.00 & 0.39 & 0.57 & 0.6 \\
\hline 14 & 15 & 4307 & 18 & 2.72 & 728100.00 & 0.75 & 0.79 & 0.5 \\
\hline 14 & 18 & 15308 & 61 & 0.80 & 4475570.00 & 0.56 & 0.56 & 0.5 \\
\hline 14 & 20 & 173 & 1 & 49.00 & 26390.00 & 1.38 & 1.61 & $0 . \varepsilon$ \\
\hline 14 & 22 & 2077 & 9 & 5.44 & 477630.00 & 0.42 & 0.46 & 0.5 \\
\hline 14 & 25 & 1327 & 14 & 3.50 & 194040.00 & 0.69 & 0.74 & 0.5 \\
\hline 15 & 6 & 346 & 2 & 24.50 & 83160.00 & 0.37 & 0.39 & 0. \\
\hline 15 & 8 & 19 & 1 & 49.00 & 18270.00 & 0.76 & 4.09 & 0. \\
\hline 15 & 9 & 77 & 1 & 49.00 & 51520.00 & 0.72 & 2.36 & 0.3 \\
\hline 15 & 11 & 19 & 1 & 49.00 & 22640.00 & 0.54 & 5.16 &. \\
\hline 15 & 14 & 4231 & 17 & 2.88 & 699040.00 & 0.76 & 0.78 & 0.5 \\
\hline 15 & 16 & 19 & 1 & 49.00 & 19040.00 & 0.42 & 2.24 & 0.1 \\
\hline 15 & 18 & 2000 & 10 & 4.90 & 416100.00 & 0.47 & 0.47 & 1.0 \\
\hline 15 & 20 & 77 & 1 & 49.00 & 48020.00 & 2.16 & 2.86 & 0.7 \\
\hline 15 & 22 & 269 & 2 & 24.50 & 72040.00 & 0.57 & 0.85 & 0.6 \\
\hline 16 & 9 & 134 & 2 & 24.50 & 38640.00 & 0.52 & 0.79 & 0.6 \\
\hline 16 & 11 & 38 & 1 & 49.00 & 31970.00 & 1.28 & 3.43 & .3 \\
\hline 16 & 15 & 19 & 1 & 49.00 & 25960.00 & 0.57 & 3.05 & 0.1 \\
\hline 16 & 18 & 481 & 5 & 9.80 & 149500.00 & 0.54 & 0.57 & 0.5 \\
\hline 16 & 23 & 19 & 1 & 49.00 & 18660.00 & 0.80 & 4.27 & 0.7 \\
\hline 17 & 4 & 2 & 1 & 49.00 & 22560.00 & 0.35 & 17.62 & 0.0 \\
\hline 17 & 11 & 38 & 1 & 49.00 & 19000.00 & 0.97 & 2.60 & $0.3^{7}$ \\
\hline 17 & 18 & 154 & 2 & 24.50 & 61180.00 & 0.76 & 1.01 & 0.7 \\
\hline 17 & 21 & 7 & 1 & 49.00 & 25830.00 & 0.79 & 11.50 & 0.0 \\
\hline 18 & 1 & 846 & 5 & 9.80 & 261850.00 & 0.72 & 0.86 & 0.8 \\
\hline 18 & 2 & 3730 & 19 & 2.58 & 1292760.00 & 0.73 & 0.75 & 0.5 \\
\hline 18 & 3 & 577 & 6 & 8.17 & 153180.00 & 0.52 & 0.55 & 0.5 \\
\hline 18 & 4 & 462 & 5 & 9.80 & 83650.00 & 0.75 & 0.83 & 0.9 \\
\hline 18 & 5 & 596 & 6 & 8.17 & 127920.00 & 0.58 & 0.59 & 0.9 \\
\hline 18 & 6 & 8153 & 33 & 1.48 & 1290630.00 & 0.61 & 0.62 & 0.5 \\
\hline 18 & 7 & 827 & 9 & 5.44 & 222750.00 & 0.47 & 0.52 & 0.5 \\
\hline 18 & 9 & 1348 & 7 & 7.00 & 165060.00 & 0.56 & 0.58 & 0.5 \\
\hline 18 & 10 & 1788 & 18 & 2.72 & 342180.00 & 0.33 & 0.34 & $0.5-5$ \\
\hline 18 & 11 & 1538 & 9 & 5.44 & 300060.00 & 0.48 & 0.51 & 0.9 \\
\hline 18 & 12 & 173 & 2 & 24.50 & 30080.00 & 0.60 & 0.71 & $0.8-8$ \\
\hline 18 & 13 & 327 & 4 & 12.25 & 64160.00 & 0.82 & 1.03 & $0.8-8$ \\
\hline 18 & 14 & 15058 & 60 & 0.82 & 4606800.00 & 0.58 & 0.59 & 0.9 \\
\hline 18 & 15 & 1942 & 10 & 4.90 & 537100.00 & 0.61 & 0.63 & 0.9 \\
\hline 18 & 16 & 481 & 5 & 9.80 & 122650.00 & 0.44 & 0.47 & 0.5 \\
\hline 18 & 17 & 173 & 2 & 24.50 & 40900.00 & 0.51 & 0.60 & $0 . \varepsilon$ \\
\hline 18 & 20 & 1942 & 10 & 4.90 & 562100.00 & 0.62 & 0.64 & 0.5 \\
\hline 18 & 22 & 1308 & 7 & 7.00 & 391720.00 & 0.41 & 0.45 & 0.9 \\
\hline 18 & 23 & 308 & 4 & 12.25 & 116120.00 & 0.39 & 0.51 & 0.7 \\
\hline
\end{tabular}


APPENDIX D:

EXISTING NETWORK ANALYSIS

\begin{tabular}{|c|c|c|c|c|c|c|c|c|}
\hline 18 & 24 & 231 & 3 & 16.33 & 54900.00 & 0.61 & 0.81 & 0.7 \\
\hline 18 & 25 & 192 & 2 & 24.50 & 50960.00 & 0.46 & 0.49 & 0. \\
\hline 19 & 2 & 115 & 2 & 24.50 & 35360.00 & 0.58 & 1.03 & 0 \\
\hline 19 & 7 & 154 & 2 & 24.50 & 25500.00 & 0.79 & 1.05 & 0. \\
\hline 19 & 10 & 154 & 2 & 24.50 & 25740.00 & 0.84 & 1.11 & 0 \\
\hline 20 & 2 & 308 & 2 & 24.50 & 67880.00 & 0.73 & 0.96 & 0. \\
\hline 20 & 14 & 96 & 1 & 49.00 & 29500.00 & 1.54 & 3.23 & 0. \\
\hline 20 & 15 & 58 & 1 & 49.00 & 31240.00 & 1.40 & 2.47 & 0.5 \\
\hline 20 & 18 & 1980 & 10 & 4.90 & 536600.00 & 0.59 & 0.60 & 0. \\
\hline 21 & 3 & 17 & 1 & 49.00 & 14740.00 & 0.97 & 5.82 & 0. \\
\hline 21 & 11 & 7 & 1 & 49.00 & 21340.00 & 0.72 & 10.48 & 0. \\
\hline 21 & 17 & 7 & 1 & 49.00 & 23310.00 & 0.71 & 10.37 & 0. \\
\hline 21 & 22 & 83 & 1 & 49.00 & 20790.00 & 0.64 & 0.79 & 0. \\
\hline 22 & 8 & 38 & 1 & 49.00 & 15630.00 & 0.88 & 2.35 & 0.3 \\
\hline 22 & 9 & 38 & 1 & 49.00 & 19550.00 & 0.41 & 1.10 & 0.3 \\
\hline 22 & 11 & 19 & 1 & 49.00 & 44950.00 & 0.57 & 7.73 & 0. \\
\hline 22 & 14 & 1980 & 8 & 6.12 & 421120.00 & 0.42 & 0.42 & 0.5 \\
\hline 22 & 15 & 308 & 2 & 24.50 & 69580.00 & 0.55 & 0.72 & 0.7 \\
\hline 22 & 18 & 1231 & 7 & 7.00 & 334950.00 & 0.35 & 0.41 & 0.8 \\
\hline 22 & 21 & 83 & 1 & 49.00 & 13160.00 & 0.41 & 0.50 & 0. \\
\hline 22 & 23 & 154 & 2 & 24.50 & 28500.00 & 0.71 & 0.93 & 0.7 \\
\hline 23 & 3 & 19 & 1 & 49.00 & 11250.00 & 0.70 & 3.75 & 0. \\
\hline 23 & 9 & 19 & 1 & 49.00 & 19200.00 & 0.36 & 1.91 & 0. \\
\hline 23 & 16 & 19 & 1 & 49.00 & 10680.00 & 0.46 & 2.44 & 0.1 \\
\hline 23 & 18 & 269 & 3 & 16.33 & 66840.00 & 0.30 & 0.34 & 0.8 \\
\hline 23 & 22 & 154 & 2 & 24.50 & 26600.00 & 0.66 & 0.87 & 0.7 \\
\hline 24 & 5 & 65 & 1 & 49.00 & 10720.00 & 0.71 & 1.11 & 0.6 \\
\hline 24 & 18 & 212 & 3 & 16.33 & 53040.00 & 0.59 & 0.85 & 0.6 \\
\hline 25 & 14 & 1308 & 13 & 3.77 & 193440.00 & 0.74 & 0.75 & 0.9 \\
\hline 25 & 18 & 154 & 2 & 24.50 & 44160.00 & 0.40 & 0.53 & $\cdot$ \\
\hline
\end{tabular}

System Flights $=778$ System Cost $=32989960.00 \quad$ System Delay $=3736.87$ System Seat-Mi Cost $=0.56$ System Pax-Mi Cost $=0.60$

Average Delay $=26.69$ system Load Factor $=0.94$

Average Seat-Mi Cost $=0.66$ Average $\mathrm{Pax}-\mathrm{Mi}$ cost $=2.54$

Average Load Factor $=0.66$

Available Seat-Mile $=58683524$ Passenger-Mile Flown $=54875896$ 
APPENDIX D:

EFFICIENT AIRLINE NETWORK ANALYSIS

\section{ANALYSIS FILE EFFICIENT AIRLINE NETWORK}

\begin{tabular}{|c|c|c|c|c|c|c|c|c|}
\hline \multicolumn{2}{|c|}{ Link } & \multirow{2}{*}{$\begin{array}{l}\text { Total } \\
\text { Flow }\end{array}$} & \multirow{2}{*}{$\begin{array}{l}\text { Total } \\
\text { Flights }\end{array}$} & \multirow{2}{*}{$\begin{array}{l}\text { Schedule } \\
\text { Delay }\end{array}$} & \multirow{2}{*}{$\begin{array}{l}\text { Total } \\
\text { Cost }\end{array}$} & \multirow{2}{*}{$\begin{array}{l}\text { Cost per } \\
\text { Seat Mile }\end{array}$} & \multirow{2}{*}{$\begin{array}{l}\text { Cost per } \\
\text { Pax Mile }\end{array}$} & \multirow{2}{*}{$\begin{array}{l}\text { Load } \\
\text { Eactor }\end{array}$} \\
\hline Fr & To & & & & & & & \\
\hline 1 & 14 & 406 & 2 & 24.50 & 74260.00 & 1.36 & 1.73 & 0.79 \\
\hline 1 & 18 & 808 & 5 & 9.80 & 216150.00 & 0.59 & 0.74 & 0.80 \\
\hline 2 & 14 & 3172 & 16 & 3.06 & 730720.00 & 0.79 & 0.81 & 0.98 \\
\hline 2 & 18 & 3596 & 18 & 2.72 & 1057320.00 & 0.63 & 0.64 & 0.99 \\
\hline 2 & 19 & 77 & 1 & 49.00 & 15680.00 & 0.52 & 0.68 & 0.75 \\
\hline 2 & 20 & 212 & 2 & 24.50 & 60600.00 & 0.66 & 1.25 & 0.52 \\
\hline 3 & 11 & 58 & 1 & 49.00 & 18750.00 & 1.00 & 1.76 & 0.57 \\
\hline 3 & 14 & 173 & 2 & 24.50 & 57120.00 & 0.51 & 0.60 & 0.85 \\
\hline 3 & 18 & 615 & 7 & 7.00 & 228620.00 & 0.66 & 0.77 & 0.86 \\
\hline 3 & 21 & 33 & 1 & 49.00 & 9380.00 & 0.62 & 1.91 & 0.32 \\
\hline 4 & 18 & 389 & 4 & 12.25 & 88880.00 & 0.99 & 1.04 & 0.95 \\
\hline 5 & 14 & 387 & 4 & 12.25 & 70320.00 & 0.76 & 0.80 & 0.95 \\
\hline 5 & 18 & 680 & 7 & 7.00 & 143080.00 & 0.56 & 0.58 & 0.95 \\
\hline 6 & 2 & 538 & 3 & 16.33 & 262080.00 & 0.49 & 0.66 & 0.73 \\
\hline 6 & 5 & 77 & 1 & 49.00 & 28800.00 & 0.43 & 0.57 & 0.75 \\
\hline 6 & 7 & 96 & 1 & 49.00 & 28280.00 & 0.39 & 0.42 & 0.94 \\
\hline 6 & 9 & 173 & 2 & 24.50 & 43500.00 & 0.55 & 0.64 & 0.85 \\
\hline 6 & 10 & 192 & 2 & 24.50 & 65240.00 & 0.40 & 0.42 & 0.94 \\
\hline 6 & 14 & 4231 & 17 & 2.88 & 1645600.00 & 0.50 & 0.51 & 0.98 \\
\hline 6 & 15 & 346 & 2 & 24.50 & 111840.00 & 0.50 & 0.52 & 0.96 \\
\hline 6 & 18 & 7865 & 32 & 1.53 & 1240320.00 & 0.60 & 0.61 & 0.98 \\
\hline 7 & 14 & 577 & 6 & 8.17 & 144720.00 & 0.61 & 0.64 & 0.94 \\
\hline 7 & 18 & 808 & 8 & 6.12 & 191760.00 & 0.46 & 0.46 & 0.99 \\
\hline 7 & 19 & 192 & 2 & 24.50 & 24480.00 & 0.76 & 0.81 & 0.94 \\
\hline 8 & 14 & 115 & 2 & 24.50 & 49000.00 & 0.74 & 1.32 & 0.56 \\
\hline 8 & 22 & 76 & 1 & 49.00 & 16030.00 & 0.90 & 1.21 & 0.75 \\
\hline 9 & 14 & 942 & 5 & 9.80 & 255600.00 & 0.57 & 0.61 & 0.94 \\
\hline 9 & 18 & 1468 & 8 & 6.12 & 172720.00 & 0.51 & 0.56 & 0.91 \\
\hline 10 & 14 & 1866 & 19 & 2.58 & 433200.00 & 0.67 & 0.69 & 0.96 \\
\hline 10 & 18 & 1962 & 20 & 2.45 & 504199.97 & 0.44 & 0.45 & 0.96 \\
\hline 10 & 19 & 77 & 1 & 49.00 & 17280.00 & 1.13 & 1.50 & 0.75 \\
\hline 11 & 3 & 63 & 1 & 49.00 & 15120.00 & 0.81 & 1.30 & 0.62 \\
\hline 11 & 14 & 403 & 3 & 16.33 & 104850.00 & 0.49 & 0.66 & 0.75 \\
\hline 11 & 16 & 153 & 2 & 24.50 & 33640.00 & 0.67 & 0.90 & 0.75 \\
\hline 11 & 17 & 38 & 1 & 49.00 & 12920.00 & 0.66 & 1.77 & 0.37 \\
\hline 11 & 18 & 1486 & 9 & 5.44 & 302850.00 & 0.48 & 0.53 & 0.92 \\
\hline 12 & 18 & 154 & 2 & 24.50 & 27840.00 & 0.56 & 0.74 & 0.75 \\
\hline 13 & 14 & 77 & 1 & 49.00 & 32640.00 & 0.45 & 0.59 & 0.75 \\
\hline 13 & 18 & 269 & 3 & 16.33 & 47790.00 & 0.82 & 0.93 & 0.88 \\
\hline 14 & 1 & 444 & 2 & 24.50 & 74480.00 & 1.36 & 1.58 & 0.86 \\
\hline 14 & 2 & 3154 & 16 & 3.06 & 655360.00 & 0.71 & 0.73 & 0.98 \\
\hline 14 & 3 & 173 & 2 & 24.50 & 55080.00 & 0.49 & 0.58 & 0.85 \\
\hline 14 & 5 & 406 & 4 & 12.25 & 72120.00 & 0.78 & 0.78 & 1.00 \\
\hline 14 & 6 & 4327 & 18 & 2.72 & 1558080.00 & 0.45 & 0.47 & 0.95 \\
\hline
\end{tabular}


APPENDIX D:

EFFICIENT AIRLINE NETWORK ANALYSIS

\begin{tabular}{|c|c|c|c|c|c|c|c|c|}
\hline 14 & 7 & 750 & 8 & 6.12 & 172480.00 & 0.54 & 0.59 & 0.92 \\
\hline 14 & 8 & 96 & 1 & 49.00 & 24640.00 & 0.75 & 0.79 & 0.94 \\
\hline 14 & 9 & 962 & 5 & 9.80 & 210800.00 & 0.47 & 0.49 & 0.96 \\
\hline 14 & 10 & 1673 & 17 & 2.88 & 345440.00 & 0.59 & 0.61 & 0.96 \\
\hline 14 & 11 & 499 & 3 & 16.33 & 81720.00 & 0.39 & 0.42 & 0.92 \\
\hline 14 & 15 & 4326 & 18 & 2.72 & 728100.00 & 0.75 & 0.79 & 0.95 \\
\hline 14 & 18 & 15289 & 61 & 0.80 & 4475570.00 & 0.56 & 0.56 & 0.99 \\
\hline 14 & 20 & 250 & 2 & 24.50 & 52780.00 & 1.38 & 2.22 & 0.62 \\
\hline 14 & 22 & 1942 & 8 & 6.12 & 424560.00 & 0.42 & 0.43 & 0.97 \\
\hline 14 & 23 & 96 & 1 & 49.00 & 35820.00 & 0.51 & 0.54 & 0.94 \\
\hline 14 & 24 & 58 & 1 & 49.00 & 25080.00 & 0.65 & 1.15 & 0.57 \\
\hline 14 & 25 & 1327 & 14 & 3.50 & 194040.00 & 0.69 & 0.74 & 0.93 \\
\hline 15 & 6 & 346 & 2 & 24.50 & 83160.00 & 0.37 & 0.39 & 0.96 \\
\hline 15 & 14 & 4327 & 18 & 2.72 & 740160.00 & 0.76 & 0.80 & 0.95 \\
\hline 15 & 18 & 2096 & 11 & 4.45 & 457710.00 & 0.47 & 0.50 & 0.95 \\
\hline 15 & 22 & 288 & 2 & 24.50 & 72040.00 & 0.57 & 0.79 & 0.72 \\
\hline 16 & 18 & 691 & 7 & 7.00 & 209300.00 & 0.54 & 0.55 & 0.97 \\
\hline 17 & 11 & 38 & 1 & 49.00 & 19000.00 & 0.97 & 2.60 & 0.37 \\
\hline 17 & 18 & 163 & 2 & 24.50 & 61180.00 & 0.76 & 0.96 & 0.80 \\
\hline 18 & 1 & 846 & 5 & 9.80 & 261850.00 & 0.72 & 0.86 & 0.84 \\
\hline 18 & 2 & 3192 & 16 & 3.06 & 1088640.00 & 0.73 & 0.74 & 0.99 \\
\hline 18 & 3 & 632 & 7 & 7.00 & 178710.00 & 0.52 & 0.59 & 0.89 \\
\hline 18 & 4 & 464 & 5 & 9.80 & 83650.00 & 0.75 & 0.82 & 0.91 \\
\hline 18 & 5 & 603 & 6 & 8.17 & 127920.00 & 0.58 & 0.59 & 0.99 \\
\hline 18 & 6 & 8596 & 35 & 1.40 & 1368850.00 & 0.61 & 0.62 & 0.98 \\
\hline 18 & 7 & 731 & 8 & 6.12 & 198000.00 & 0.47 & 0.53 & 0.90 \\
\hline 18 & 9 & 1526 & 8 & 6.12 & 188640.00 & 0.56 & 0.59 & 0.95 \\
\hline 18 & 10 & 1673 & 17 & 2.88 & 323170.00 & 0.33 & 0.34 & 0.96 \\
\hline 18 & 11 & 1606 & 9 & 5.44 & 300060.00 & 0.48 & 0.48 & 0.99 \\
\hline 18 & 12 & 173 & 2 & 24.50 & 30080.00 & 0.60 & 0.71 & 0.85 \\
\hline 18 & 13 & 327 & 4 & 12.25 & 64160.00 & 0.82 & 1.03 & 0.80 \\
\hline 18 & 14 & 15077 & 60 & 0.82 & 4606800.00 & 0.58 & 0.59 & 0.99 \\
\hline 18 & 15 & 2038 & 11 & 4.45 & 590810.00 & 0.61 & 0.66 & 0.92 \\
\hline 18 & 16 & 538 & 6 & 8.17 & 147180.00 & 0.44 & 0.50 & 0.88 \\
\hline 18 & 17 & 184 & 2 & 24.50 & 40900.00 & 0.51 & 0.57 & 0.90 \\
\hline 18 & 19 & 115 & 2 & 24.50 & 39000.00 & 0.41 & 0.73 & 0.56 \\
\hline 18 & 20 & 1942 & 10 & 4.90 & 562100.00 & 0.62 & 0.64 & 0.97 \\
\hline 18 & 21 & 90 & 1 & 49.00 & 32160.00 & 0.45 & 0.51 & 0.88 \\
\hline 18 & 22 & 1352 & 7 & 7.00 & 391720.00 & 0.41 & 0.43 & 0.96 \\
\hline 18 & 23 & 369 & 4 & 12.25 & 116120.00 & 0.39 & 0.43 & 0.90 \\
\hline 18 & 24 & 238 & 3 & 16.33 & 54900.00 & 0.61 & 0.79 & 0.78 \\
\hline 18 & 25 & 192 & 2 & 24.50 & 50960.00 & 0.46 & 0.49 & 0.94 \\
\hline 19 & 2 & 115 & 2 & 24.50 & 35360.00 & 0.58 & 1.03 & 0.56 \\
\hline 19 & 7 & 19 & 1 & 49.00 & 12750.00 & 0.79 & 4.25 & 0.19 \\
\hline 19 & 10 & 212 & 3 & 16.33 & 38610.00 & 0.84 & 1.21 & 0.69 \\
\hline 19 & 18 & 96 & 1 & 49.00 & 24000.00 & 0.51 & 0.54 & 0.94 \\
\hline 20 & 2 & 346 & 2 & 24.50 & 67880.00 & 0.73 & 0.86 & 0.86 \\
\hline 20 & 15 & 58 & 1 & 49.00 & 31240.00 & 1.40 & 2.47 & 0.57 \\
\hline 20 & 18 & 2038 & 11 & 4.45 & 590260.00 & 0.59 & 0.64 & 0.92 \\
\hline 21 & 18 & 114 & 2 & 24.50 & 84420.00 & 0.59 & 1.06 & 0.56 \\
\hline 22 & 8 & 76 & 1 & 49.00 & 15630.00 & 0.88 & 1.18 & 0.75 \\
\hline 22 & 14 & 1884 & 8 & 6.12 & 421120.00 & 0.42 & 0.44 & 0.95 \\
\hline 22 & 15 & 327 & 2 & 24.50 & 69580.00 & 0.55 & 0.68 & 0.81 \\
\hline 22 & 18 & 1236 & 7 & 7.00 & 334950.00 & 0.35 & 0.40 & 0.88 \\
\hline 22 & 23 & 58 & 1 & 49.00 & 14250.00 & 0.71 & 1.24 & 0.57 \\
\hline 23 & 14 & 96 & 1 & 49.00 & 35820.00 & 0.51 & 0.54 & 0.94 \\
\hline 23 & 18 & 326 & 4 & 12.25 & 89120.00 & 0.30 & 0.37 & 0.80 \\
\hline 23 & 22 & 58 & 1 & 49.00 & 13300.00 & 0.66 & 1.16 & 0.57 \\
\hline 24 & 14 & 58 & 1 & 49.00 & 22440.00 & 0.58 & 1.03 & 0.57 \\
\hline 24 & 18 & 219 & 3 & 16.33 & 53040.00 & 0.59 & 0.83 & 0.72 \\
\hline
\end{tabular}


APPENDIX D:

EFFICIENT AIRLINE NETWORK ANALYSIS

\begin{tabular}{|c|c|c|c|c|c|c|c|}
\hline $\begin{array}{ll}25 & 14 \\
25 & 18\end{array}$ & $\begin{array}{r}1308 \\
154\end{array}$ & 13 & $\begin{array}{r}3.77 \\
2450\end{array}$ & 193440.00 & 0.74 & 0.75 & \\
\hline 2518 & & 2 & & 44160.00 & 0.40 & 0.53 & 0.75 \\
\hline
\end{tabular}

System Flights $=745$ System Cost $=32449630.00$ System Delay $=2160.43$ System Seat-Mi Cost $=0.56$ system Pax-Mi Cost $=0.59$

Average Delay $=20.38$ System Load Factor $=0.95$

Average Seat-Mi Cost $=0.63$ Average Pax-Mi cost $=0.84$

Average Load Factor $=0.83$

Available Seat-Mile $=58069236$ Passenger-Mile Flown $=55231064$ 
ANALYSIS FILE EFFICIENT PASSENGERS NETWORK

\begin{tabular}{|c|c|c|c|c|c|c|c|c|}
\hline \multicolumn{2}{|c|}{ Link } & Total & Total & Schedule & Total & Cost per & Cost per & Load \\
\hline Fr & To & Flow & Flights & Delay & Cost & Seat Mile & Pax Mile & Facto \\
\hline 1 & 18 & 1214 & 7 & 7.00 & 302610.00 & 0.59 & 0.69 & 0.86 \\
\hline 2 & 14 & 3211 & 16 & 3.06 & 730720.00 & 0.79 & 0.80 & 0.99 \\
\hline 2 & 18 & 3808 & 19 & 2.58 & 1116060.00 & 0.63 & 0.64 & 0.99 \\
\hline 3 & 18 & 846 & 9 & 5.44 & 293940.00 & 0.66 & 0.72 & 0.92 \\
\hline 3 & 21 & 105 & 2 & 24.50 & 18760.00 & 0.62 & 1.20 & 0.51 \\
\hline 4 & 18 & 389 & 4 & 12.25 & 88880.00 & 0.99 & 1.04 & 0.95 \\
\hline 5 & 18 & 1067 & 11 & 4.45 & 224840.00 & 0.56 & 0.58 & 0.95 \\
\hline 6 & 14 & 4577 & 19 & 2.58 & 1839200.00 & 0.50 & 0.53 & 0.95 \\
\hline 6 & 18 & 8941 & 36 & 1.36 & 1395360.00 & 0.60 & 0.61 & 0.99 \\
\hline 7 & 14 & 615 & 7 & 7.00 & 168840.00 & 0.61 & 0.71 & 0.86 \\
\hline 7 & 18 & 827 & 9 & 5.44 & 215730.00 & 0.46 & 0.51 & 0.90 \\
\hline 8 & 14 & 191 & 2 & 24.50 & 49000.00 & 0.74 & 0.79 & 0.94 \\
\hline 9 & 18 & 2410 & 12 & 4.08 & 259080.00 & 0.51 & 0.51 & 1.00 \\
\hline 10 & 14 & 1789 & 18 & 2.72 & 410400.00 & 0.67 & 0.68 & 0.97 \\
\hline 10 & 18 & 1981 & 20 & 2.45 & 504199.97 & 0.44 & 0.45 & 0.97 \\
\hline 11 & 18 & 2023 & 12 & 4.08 & 403800.03 & 0.48 & 0.52 & 0.94 \\
\hline 11 & 21 & 5 & 1 & 49.00 & 16950.00 & 0.57 & 11.65 & 0.05 \\
\hline 12 & 18 & 154 & 2 & 24.50 & 27840.00 & 0.56 & 0.74 & 0.75 \\
\hline 13 & 18 & 346 & 4 & 12.25 & 63720.00 & 0.82 & 0.96 & 0.85 \\
\hline 14 & 2 & 3269 & 17 & 2.88 & 696320.00 & 0.71 & 0.74 & 0.95 \\
\hline 14 & 6 & 4673 & 19 & 2.58 & 1644640.00 & 0.45 & 0.46 & 0.97 \\
\hline 14 & 7 & 615 & 7 & 7.00 & 150920.00 & 0.54 & 0.63 & 0.86 \\
\hline 14 & 8 & 172 & 2 & 24.50 & 49280.00 & 0.75 & 0.88 & 0.84 \\
\hline 14 & 10 & 1731 & 17 & 2.88 & 345440.00 & 0.59 & 0.59 & 1.00 \\
\hline 14 & 15 & 4980 & 20 & 2.45 & 809000.00 & 0.75 & 0.76 & 0.98 \\
\hline 14 & 18 & 18250 & 73 & 0.67 & 5356010.00 & 0.56 & 0.56 & 0.99 \\
\hline 14 & 19 & 442 & 5 & 9.80 & 149600.00 & 0.50 & 0.58 & 0.87 \\
\hline 14 & 22 & 2230 & 9 & 5.44 & 477630.00 & 0.42 & 0.43 & 1.00 \\
\hline 14 & 25 & 1519 & 15 & 3.27 & 207900.00 & 0.69 & 0.69 & 0.99 \\
\hline 15 & 14 & 4865 & 20 & 2.45 & 822400.00 & 0.76 & 0.79 & 0.96 \\
\hline 15 & 18 & 2192 & 11 & 4.45 & 457710.00 & 0.47 & 0.47 & 0.99 \\
\hline 16 & 18 & 691 & 7 & 7.00 & 209300.00 & 0.54 & 0.55 & 0.97 \\
\hline 17 & 18 & 194 & 2 & 24.50 & 61180.00 & 0.76 & 0.80 & 0.95 \\
\hline 17 & 21 & 7 & 1 & 49.00 & 25830.00 & 0.79 & 11.50 & 0.07 \\
\hline 18 & 1 & 1290 & 7 & 7.00 & 366590.00 & 0.72 & 0.79 & 0.92 \\
\hline 18 & 2 & 4038 & 20 & 2.45 & 1360800.00 & 0.73 & 0.73 & 1.00 \\
\hline 18 & 3 & 923 & 10 & 4.90 & 255300.00 & 0.52 & 0.57 & 0.90 \\
\hline 18 & 4 & 464 & 5 & 9.80 & 83650.00 & 0.75 & 0.82 & 0.91 \\
\hline 18 & 5 & 1086 & 11 & 4.45 & 234520.00 & 0.58 & 0.60 & 0.97 \\
\hline 18 & 6 & 8596 & 35 & 1.40 & 1368850.00 & 0.61 & 0.62 & 0.98 \\
\hline 18 & 7 & 846 & 9 & 5.44 & 222750.00 & 0.47 & 0.51 & 0.92 \\
\hline 18 & 9 & 2661 & 14 & 3.50 & 330120.00 & 0.56 & 0.59 & 0.95 \\
\hline 18 & 10 & 1884 & 19 & 2.58 & 361190.00 & 0.33 & 0.34 & 0.97 \\
\hline 18 & 11 & 2079 & 12 & 4.08 & 400080.00 & 0.48 & 0.50 & 0.96 \\
\hline 18 & 12 & 173 & 2 & 24.50 & 30080.00 & 0.60 & 0.71 & 0.85 \\
\hline 18 & 13 & 327 & 4 & 12.25 & 64160.00 & 0.82 & 1.03 & 0.80 \\
\hline 18 & 14 & 17941 & 71 & 0.69 & 5451380.00 & 0.58 & 0.58 & 1.00 \\
\hline 18 & 15 & 2115 & 11 & 4.45 & 590810.00 & 0.61 & 0.63 & 0.96 \\
\hline 18 & 16 & 691 & 7 & 7.00 & 171710.00 & 0.44 & 0.45 & 0.97 \\
\hline 18 & 17 & 215 & 3 & 16.33 & 61350.00 & 0.51 & 0.73 & 0.70 \\
\hline
\end{tabular}


APPENDIX D:

EFEICIENT PASSENGERS NETWORK ANALYSIS

\begin{tabular}{|c|c|c|c|c|c|c|c|}
\hline 1820 & 2404 & 12 & 4.08 & 674520.00 & 0.62 & 0.62 & 1.00 \\
\hline 1822 & 1404 & 7 & 7.00 & 391720.00 & 0.41 & 0.42 & 1.00 \\
\hline 1823 & 523 & 6 & 8.17 & 174180.00 & 0.39 & 0.45 & 0.8 \\
\hline 1824 & 296 & 3 & 16.33 & 54900.00 & 0.61 & 0.63 & 0.5 \\
\hline $19 \quad 14$ & 423 & 5 & 9.80 & 149600.00 & 0.50 & 0.60 & 0.8 \\
\hline $20 \quad 18$ & 2442 & 13 & 3.77 & 697580.00 & 0.59 & 0.63 & 0.9 \\
\hline 21 & 17 & 1 & 49.00 & 14740.00 & 0.97 & 5.82 & 0.1 \\
\hline 2111 & 7 & 1 & 49.00 & 21340.00 & 0.72 & 10.48 & 0.0 \\
\hline $21 \quad 17$ & 7 & 1 & 49.00 & 23310.00 & 0.71 & 10.37 & 0.0 \\
\hline 2118 & 77 & 1 & 49.00 & 42210.00 & 0.59 & 0.78 & 0.7 \\
\hline 2122 & 6 & 1 & 49.00 & 20790.00 & 0.64 & 10.93 & 0.0 \\
\hline $22 \quad 14$ & 2134 & 9 & 5.44 & 473760.00 & 0.42 & 0.44 & 0.9 \\
\hline 2218 & 1365 & 7 & 7.00 & 334950.00 & 0.35 & 0.37 & 0.9 \\
\hline 2221 & 6 & 1 & 49.00 & 13160.00 & 0.41 & 6.92 & 0.0 \\
\hline 2318 & 480 & 5 & 9.80 & 111400.00 & 0.30 & 0.32 & 0.9 \\
\hline 2418 & 277 & 3 & 16.33 & 53040.00 & 0.59 & 0.65 & 0.9 \\
\hline 2514 & 1462 & 15 & 3.27 & 223200.00 & 0.74 & 0.77 & 0.96 \\
\hline
\end{tabular}

System Flights $=766$ System Cost $=34420832.00$ System Delay $=$

843.98 System Seat-Mi Cost $=0.56$ System Pax-Mi Cost $=$
Average Delay $=12.60 \quad$ system Load Factor $=0.97$

Average Seat-Mi Cost $=0.60$ Average Pax-Mi cost $=1.58$

Average Load Factor $=0.84$

Available Seat-Mile $=61540296$ Passenger-Mile Flown $=59562624$ 
APPENDIX D:

EFFICIENT COMBINED NETWORK ANALYSIS

ANALYSIS FILE EFFICIENT COMBINED NETWORK

\begin{tabular}{|c|c|c|c|c|c|c|c|c|}
\hline \multicolumn{2}{|c|}{ Link } & \multirow{2}{*}{$\begin{array}{l}\text { Total } \\
\text { Flow }\end{array}$} & \multirow{2}{*}{$\begin{array}{l}\text { Total } \\
\text { Flights }\end{array}$} & \multirow{2}{*}{$\begin{array}{l}\text { Schedule } \\
\text { Delay }\end{array}$} & \multirow{2}{*}{$\begin{array}{l}\text { Total } \\
\text { Cost }\end{array}$} & \multirow{2}{*}{$\begin{array}{l}\text { Cost per } \\
\text { Seat Mile }\end{array}$} & \multirow{2}{*}{$\begin{array}{l}\text { Cost per } \\
\text { Pax Mile }\end{array}$} & \multirow{2}{*}{$\begin{array}{l}\text { Load } \\
\text { Factor }\end{array}$} \\
\hline Fr & To & & & & & & & \\
\hline 1 & 14 & 404 & 2 & 24.50 & 74260.00 & 1.36 & 1.73 & 0.78 \\
\hline 1 & 18 & 810 & 5 & 9.80 & 216150.00 & 0.59 & 0.74 & 0.81 \\
\hline 2 & 14 & 3134 & 16 & 3.06 & 730720.00 & 0.79 & 0.82 & 0.97 \\
\hline 2 & 18 & 3596 & 18 & 2.72 & 1057320.00 & 0.63 & 0.64 & 0.99 \\
\hline 2 & 19 & 212 & 3 & 16.33 & 47040.00 & 0.52 & 0.74 & 0.69 \\
\hline 2 & 20 & 212 & 2 & 24.50 & 60600.00 & 0.66 & 1.25 & 0.52 \\
\hline 3 & 14 & 173 & 2 & 24.50 & 57120.00 & 0.51 & 0.60 & 0.85 \\
\hline 3 & 18 & 673 & 7 & 7.00 & 228620.00 & 0.66 & 0.70 & 0.94 \\
\hline 3 & 21 & 28 & 1 & 49.00 & 9380.00 & 0.62 & 2.25 & 0.27 \\
\hline 4 & 18 & 389 & 4 & 12.25 & 88880.00 & 0.99 & 1.04 & 0.95 \\
\hline 5 & 14 & 387 & 4 & 12.25 & 70320.00 & 0.76 & 0.80 & 0.95 \\
\hline 5 & 18 & 680 & 7 & 7.00 & 143080.00 & 0.56 & 0.58 & 0.95 \\
\hline 6 & 14 & 4231 & 17 & 2.88 & 1645600.00 & 0.50 & 0.51 & 0.98 \\
\hline 6 & 18 & 9287 & 37 & 1.32 & 1434120.00 & 0.60 & 0.60 & 1.00 \\
\hline 7 & 14 & 577 & 6 & 8.17 & 144720.00 & 0.61 & 0.64 & 0.94 \\
\hline 7 & 18 & 865 & 9 & 5.44 & 215730.00 & 0.46 & 0.49 & 0.94 \\
\hline 8 & 14 & 191 & 2 & 24.50 & 49000.00 & 0.74 & 0.79 & 0.94 \\
\hline 9 & 14 & 942 & 5 & 9.80 & 255600.00 & 0.57 & 0.61 & 0.94 \\
\hline 9 & 18 & 1468 & 8 & 6.12 & 172720.00 & 0.51 & 0.56 & 0.91 \\
\hline 10 & 14 & 1731 & 17 & 2.88 & 387600.00 & 0.67 & 0.67 & 1.00 \\
\hline 10 & 18 & 2135 & 21 & 2.33 & 529410.00 & 0.44 & 0.44 & 1.00 \\
\hline 11 & 14 & 365 & 3 & 16.33 & 104850.00 & 0.49 & 0.73 & 0.68 \\
\hline 11 & 18 & 1663 & 10 & 4.90 & 336500.00 & 0.48 & 0.52 & 0.92 \\
\hline 12 & 18 & 154 & 2 & 24.50 & 27840.00 & 0.56 & 0.74 & 0.75 \\
\hline 13 & 18 & 346 & 4 & 12.25 & 63720.00 & 0.82 & 0.96 & 0.85 \\
\hline 14 & 1 & 444 & 2 & 24.50 & 74480.00 & 1.36 & 1.58 & 0.86 \\
\hline 14 & 2 & 3289 & 17 & 2.88 & 696320.00 & 0.71 & 0.74 & 0.96 \\
\hline 14 & 3 & 173 & 2 & 24.50 & 55080.00 & 0.49 & 0.58 & 0.85 \\
\hline 14 & 5 & 404 & 4 & 12.25 & 72120.00 & 0.78 & 0.79 & 0.99 \\
\hline 14 & 6 & 4327 & 18 & 2.72 & 1558080.00 & 0.45 & 0.47 & 0.95 \\
\hline 14 & 7 & 634 & 7 & 7.00 & 150920.00 & 0.54 & 0.61 & 0.89 \\
\hline 14 & 8 & 172 & 2 & 24.50 & 49280.00 & 0.75 & 0.88 & 0.84 \\
\hline 14 & 9 & 962 & 5 & 9.80 & 210800.00 & 0.47 & 0.49 & 0.96 \\
\hline 14 & 10 & 1673 & 17 & 2.88 & 345440.00 & 0.59 & 0.61 & 0.96 \\
\hline 14 & 11 & 365 & 3 & 16.33 & 81720.00 & 0.39 & 0.57 & 0.68 \\
\hline 14 & 15 & 4634 & 19 & 2.58 & 768550.00 & 0.75 & 0.78 & 0.96 \\
\hline 14 & 18 & 15808 & 63 & 0.78 & 4622310.00 & 0.56 & 0.56 & 0.99 \\
\hline 14 & 22 & 2230 & 9 & 5.44 & 477630.00 & 0.42 & 0.43 & 1.00 \\
\hline 14 & 25 & 1519 & 15 & 3.27 & 207900.00 & 0.69 & 0.69 & 0.99 \\
\hline 15 & 14 & 4519 & 18 & 2.72 & 740160.00 & 0.76 & 0.77 & 0.99 \\
\hline 15 & 18 & 2538 & 13 & 3.77 & 540930.00 & 0.47 & 0.48 & 0.97 \\
\hline 16 & 18 & 691 & 7 & 7.00 & 209300.00 & 0.54 & 0.55 & 0.97 \\
\hline 17 & 18 & 201 & 2 & 24.50 & 61180.00 & 0.76 & 0.77 & 0.99 \\
\hline 18 & 1 & 846 & 5 & 9.80 & 261850.00 & 0.72 & 0.86 & 0.84 \\
\hline 18 & 2 & 3730 & 19 & 2.58 & 1292760.00 & 0.73 & 0.75 & 0.97 \\
\hline 18 & 3 & 690 & 7 & 7.00 & 178710.00 & 0.52 & 0.54 & 0.97 \\
\hline 18 & 4 & 464 & 5 & 9.80 & 83650.00 & 0.75 & 0.82 & 0.91 \\
\hline 18 & 5 & 682 & 7 & 7.00 & 149240.00 & 0.58 & 0.61 & 0.96 \\
\hline 18 & 6 & 8942 & 36 & 1.36 & 1407960.00 & 0.61 & 0.61 & 0.99 \\
\hline 18 & 7 & 827 & 9 & 5.44 & 222750.00 & 0.47 & 0.52 & 0.90 \\
\hline
\end{tabular}


APPENDIX D:

EFFICIENT COMBINED NETWORK ANALYSIS

\begin{tabular}{|c|c|c|c|c|c|c|c|c|}
\hline 18 & 9 & 1699 & 9 & 5.44 & 212220.00 & 0.56 & 0.59 & 0.94 \\
\hline 181 & 10 & 1884 & 19 & 2.58 & 361190.00 & 0.33 & 0.34 & 0.97 \\
\hline 181 & 11 & 1721 & 10 & 4.90 & 333400.00 & 0.48 & 0.50 & 0.96 \\
\hline 181 & 12 & 173 & 2 & 24.50 & 30080.00 & 0.60 & 0.71 & 0.85 \\
\hline 181 & 13 & 327 & 4 & 12.25 & 64160.00 & 0.82 & 1.03 & 0.80 \\
\hline 181 & 14 & 15557 & 62 & 0.79 & 4760360.00 & 0.58 & 0.59 & 0.99 \\
\hline 181 & 15 & 2461 & 13 & 3.77 & 698230.00 & 0.61 & 0.64 & 0.94 \\
\hline 181 & 16 & 691 & 7 & 7.00 & 171710.00 & 0.44 & 0.45 & 0.97 \\
\hline 181 & 17 & 222 & 3 & 16.33 & 61350.00 & 0.51 & 0.70 & 0.73 \\
\hline 181 & 19 & 230 & 3 & 16.33 & 58500.00 & 0.41 & 0.55 & 0.75 \\
\hline 182 & 20 & 2192 & 11 & 4.45 & 618310.00 & 0.62 & 0.63 & 0.99 \\
\hline 182 & 21 & 95 & 1 & 49.00 & 32160.00 & 0.45 & 0.48 & 0.93 \\
\hline 182 & 22 & 1410 & 8 & 6.12 & 447680.00 & 0.41 & 0.47 & 0.88 \\
\hline 182 & 23 & 523 & 6 & 8.17 & 174180.00 & 0.39 & 0.45 & 0.85 \\
\hline 182 & 24 & 296 & 3 & 16.33 & 54900.00 & 0.61 & 0.63 & 0.97 \\
\hline 19 & 2 & 115 & 2 & 24.50 & 35360.00 & 0.58 & 1.03 & 0.56 \\
\hline 191 & 10 & 154 & 2 & 24.50 & 25740.00 & 0.84 & 1.11 & 0.75 \\
\hline 191 & 14 & 154 & 2 & 24.50 & 59840.00 & 0.50 & 0.66 & 0.75 \\
\hline 20 & 2 & 308 & 2 & 24.50 & 67880.00 & 0.73 & 0.96 & 0.76 \\
\hline 201 & 18 & 2134 & 11 & 4.45 & 590260.00 & 0.59 & 0.61 & 0.97 \\
\hline 211 & 18 & 114 & 2 & 24.50 & 84420.00 & 0.59 & 1.06 & 0.56 \\
\hline 221 & 14 & 2134 & 9 & 5.44 & 473760.00 & 0.42 & 0.44 & 0.95 \\
\hline 221 & 18 & 1371 & 7 & 7.00 & 334950.00 & 0.35 & 0.36 & 0.97 \\
\hline 231 & 18 & 480 & 5 & 9.80 & 111400.00 & 0.30 & 0.32 & 0.94 \\
\hline 241 & 18 & 277 & 3 & 16.33 & 53040.00 & 0.59 & 0.65 & 0.91 \\
\hline 1 & 14 & 1462 & 15 & 3.27 & 223200.00 & 0.74 & 0.77 & 0.96 \\
\hline
\end{tabular}

System Flights $=744$ System Cost $=32808300.00$ System Delay $=$

881.55

System Seat-Mi Cost $=0.56$ System Pax-Mi Cost $=0.58$

Average Delay $=11.60$ System Load Factor $=0.96$

Average Seat-Mi Cost $=0.61$ Average Pax-Mi cost $=0.71$

Average Load Factor $=0.89$

Available Seat-Mile $=58730592$ Passenger-Mile Flown $=56543592$ 